\title{
CONTRIBUIÇÃO AO ESTUDO DA LOCALIZAÇÃO DE DEFORMAÇÕES COM MODELOS CONSTITUTIVOS DE DANO E PLASTICIDADE
}

\section{LARISSA DRIEMEIER}

\begin{abstract}
TESE APRESENTADA À ESCOLA DE ENGENHARIA DE SÃO CARLOS, DA UNIVERSIDADE DE SÃO PAULO, COMO PARTE DOS REQUISITOS PARA OBTENÇÃO DO TÍTULO DE DOUTOR EM ENGENHARIA DE ESTRUTURAS.
\end{abstract}

ORIENTADOR: Prof. Assoc. SÉRGIO PERSIVAL BARONCINI PROENÇA

São Carlos

1999 
Class. TESE- EESC

Gutt.

952

Tombo

Ficha catalográfica preparada pela Seção de Tratamento da Informaçăo do Serviço de Biblioteca - EESC/USP

\section{D779c Driemeier, Larissa} deformações com modelos constitutivos de dano e plasticidade / Larissa Driemeier. -- São Carlos, 1999.

Tese (Doutorado) -- Escola de Engenharia de São Carlos-Universidade de São Paulo, 1999.

Area: Engenharia de Estruturas.

Orientador: Prof. Dr. Sergio Persival Baroncini Proença.

1. Mecânica do dano. 2. Plasticidade.

3. Localização. I. Título. 


\section{Candidata: Engenheira LARISSA DRIEMEIER}

Tese defendida e aprovada em 26.02.1999

pela Comissão Julgadora:

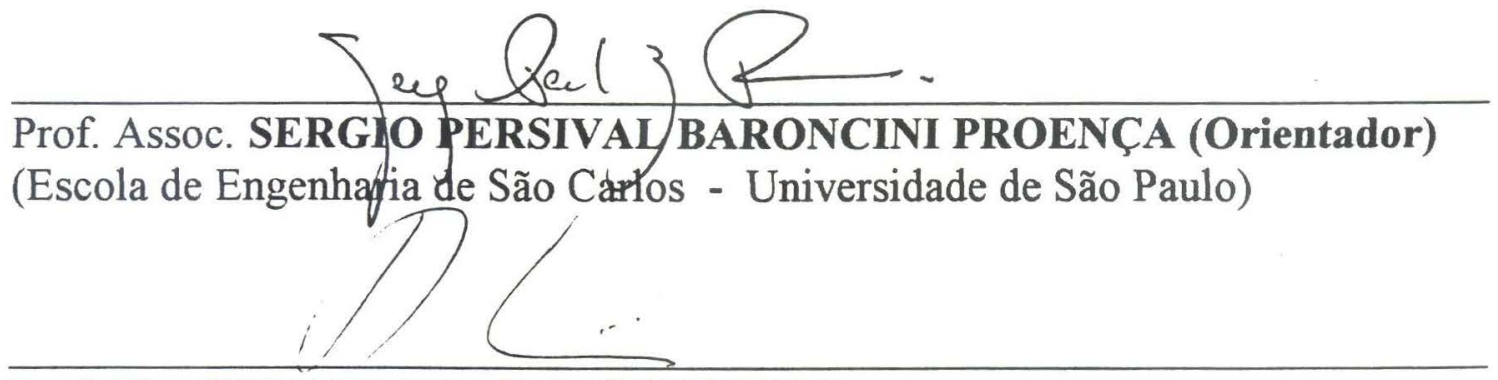

Prof. Tit. WILSON SERGIO VENTURINI

(Escola de Engenharia de São Carlos - Universidade de São Paulo)

Reharmsnernimeutra

Prof. Tit. PÁULO DE MATTOS PIMENTA

(Escola Politécnica - Universidade de São Paulo)

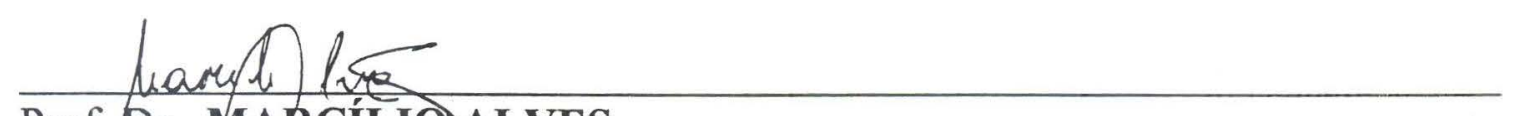

Prof.Dr. MARCÍlO ALVES

(Escola Politécnica - Universidade de São Paulo)

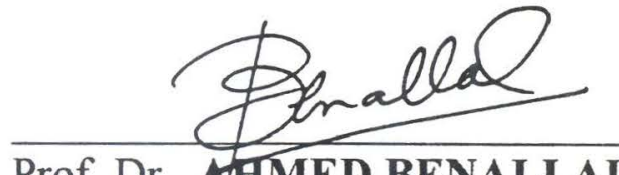

Prof. Dr. AMED BENALLAL

(Universidade de Paris VI, França)

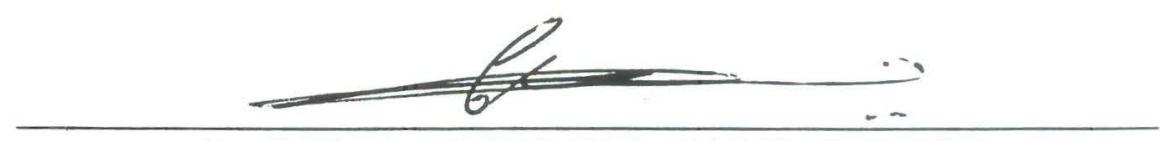

Prof. Titular CARLITO CALIL JUNIOR

Coordenador da Área de Engenharia de Estruturas

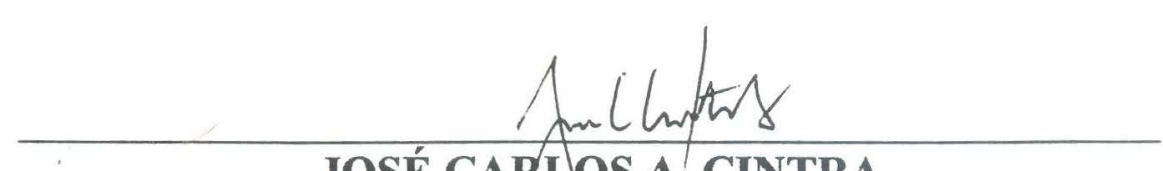

JOSÉ CARIOOS A. CINTRA

Presidente da Comissãode Pós-Graduação da EESC 
Ao Guto e à Marina, com amor. 
Agradeço ao orientador prof.Dr. Sergio Proença, pela ajuda indispensável,pela paciência e pela amizade.

Agradeço à prof ${ }^{\underline{a}}$.Dra Claudia Comi, pela dedicação, pela valiosa atenção e pelo companheirismo durante o ano de trabalho na Itália.

Agradeço ao meu marido, aos meus pais e aos meus irmãos, pelo amor,pela ajuda e compreensão.

Agradeço aos amigos por todos os momentos felizez e pelas horas difíceis compartilhadas.

Agradeço à Fapesp pelo apoio financeiro indispensável. 


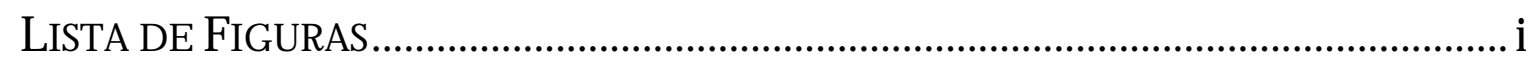

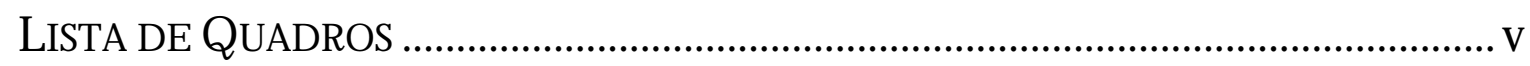

LISTA DE TABELAS ………………................................................................... vi

LISTA DE SÍMBOLOS .........................................................................................

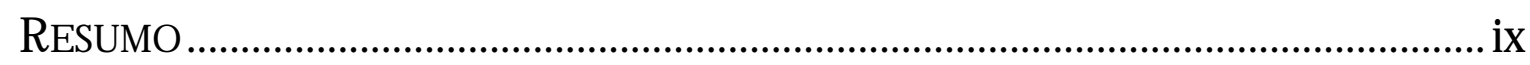

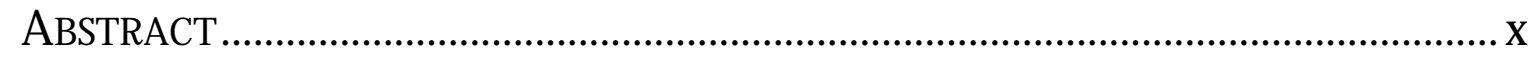

\section{INTRODUÇÃO}

1.1 ASPECTOS GERAIS E OBJETIVOS D O TRABALHO ................................................

1.2 O CONTEÚDO DOS CAPÍTULOS DA TESE ............................................................

\section{FENÔMENO DE LOCALIZAÇÃO}

2.1 APRESENTAÇÃO DO FENÔMENO DE LOCALIZAÇÃO .........................................

2.2 EXEMPLO ILUSTRATIVO DO FENÔMENO NA ANÁLISE NUMÉRICA ......................

2.2.1 Sduģãohomoĝ̂n ................................................................................................. 
2.2.2 Resposta localizada em2 2.............................................................................10

2.2.3Sdựãoloalizada em 1...............................................................................12

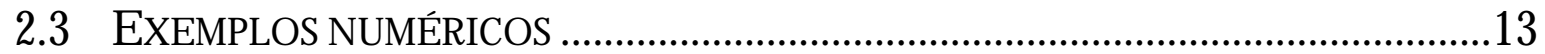

2.3.1 A nálisedbexemplounidimensional .......................................................................14

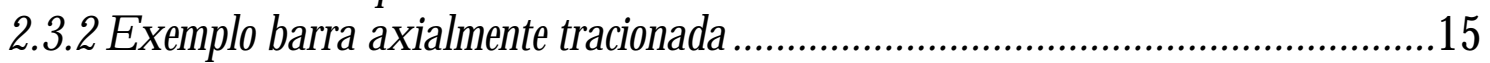

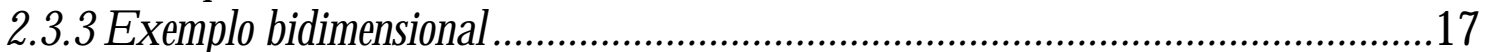

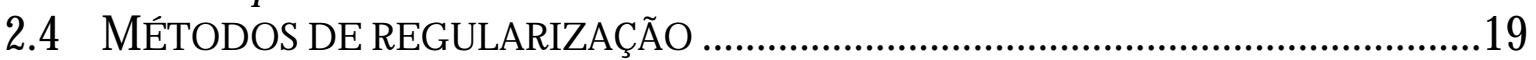

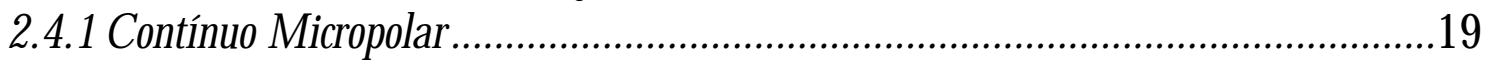

2.4.2 Integral não- local..............................................................................................20

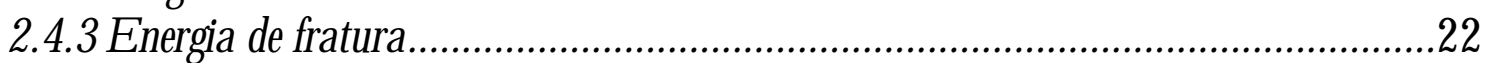

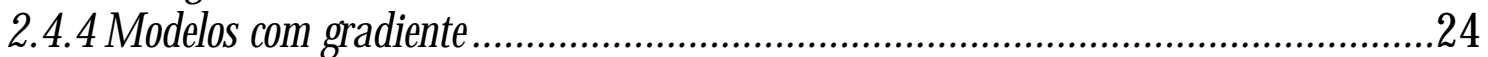

\section{MÉTODO DE REGULARIZAÇÃO COM GRADIENTES}

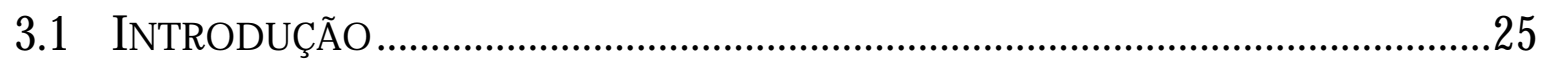

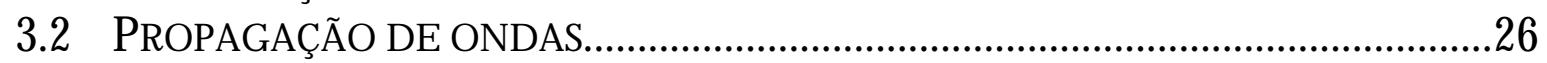

3.3 CONSIDERAÇÃO DE GRADIENTES DE ORDEM SUPERIOR ..................................33

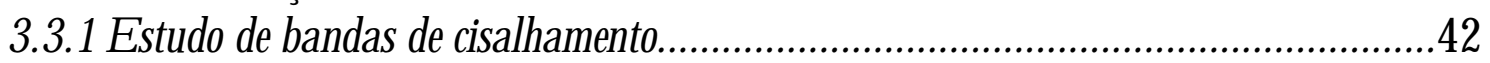

\section{MODELO DE DANO COM GRADIENTE}

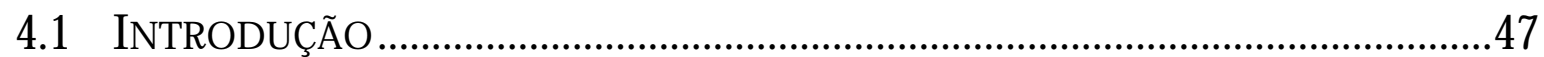

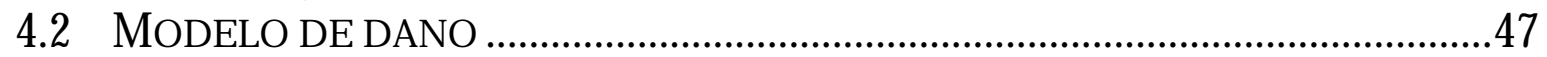

4.2.1 Localização com singularidadedbtensor aástico..............................................51

4.2.2 Propacaģãodeanda..........................................................................................56

\section{IMPLEMENTAÇÃO DO MODELO DE DANO}

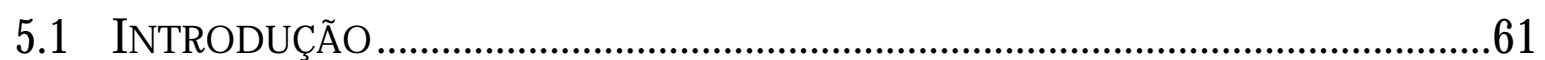

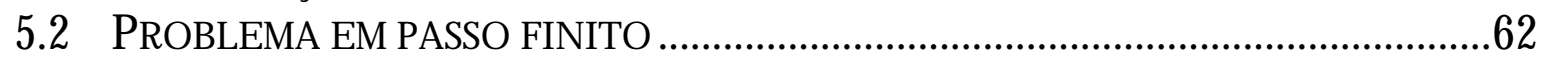

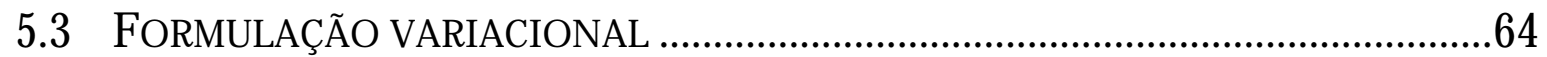

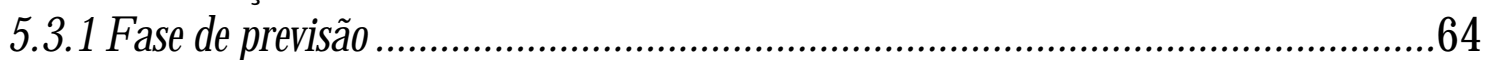

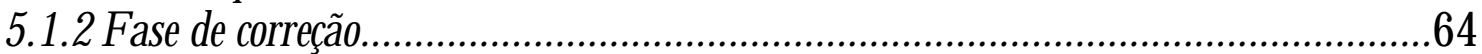

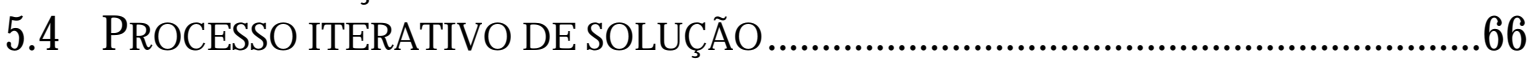

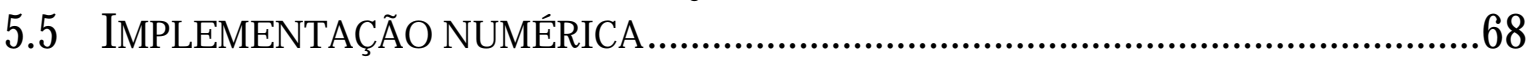

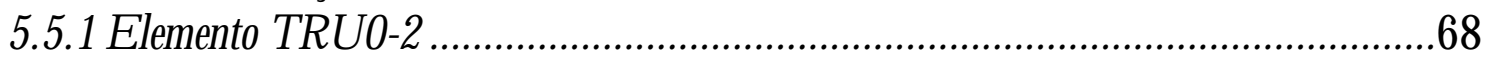

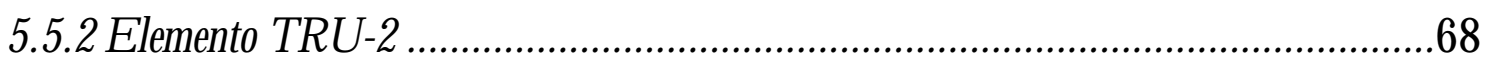

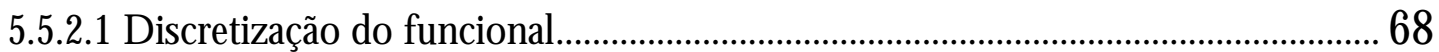

5.5.2.2 Sobre implementação ........................................................................................ 71

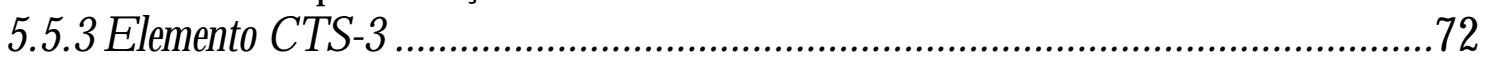




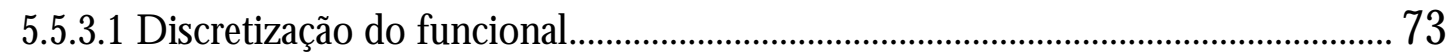

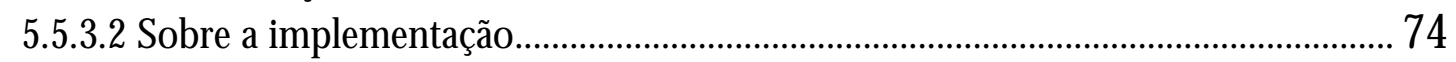

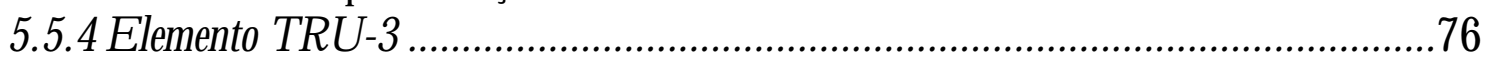

5.5.4.1 Discretização do variacional ..................................................................................... 76

\section{EXEMPLOS}

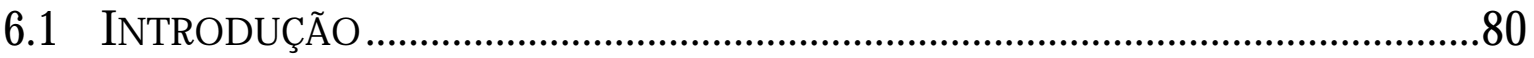

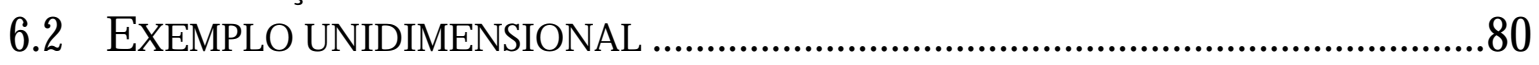

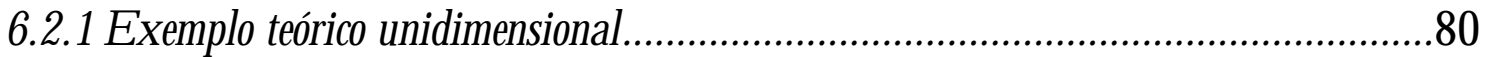

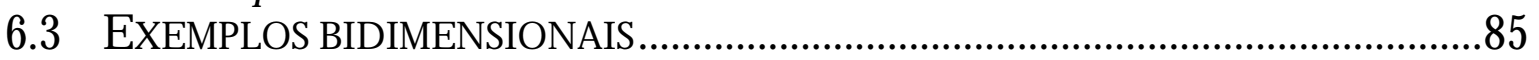

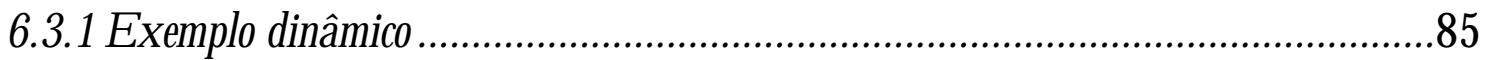

6.3.2V iga paredecomentalhe......................................................................................8

6.3.3V iga comentalhe.......................................................................................91

\section{SÍNTESE DO CONTEÚDO E CONCLUSÕES}

\section{BIBLIOGRAFIA}

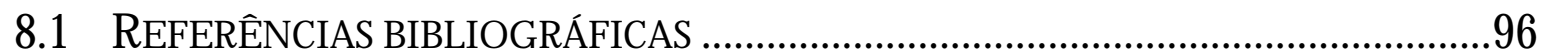

8.2 BIBLIO GRAFIA COMPLEMENTAR EM ORDEM ALFABÉTICA ............................ 100

\section{ANEXO A}

D ISCUSSÃO SO BRE PROBLEMA BEMPOSICIONADO

\section{ANEXO B}

ELEMENTOS FINITOS COM VARIÁVEIS GENERALIZADAS 
Lista de figuras

\section{Capítulo 02}

Figura 2.1. (a) Fissuração em estágio inicial de solicitação: distribuída ao longo da estrutura;

(b) Localização do fenômeno de fissuração;

(c) Formação da macrofissura. 5

Figura 2.2. Dados geométricos da barra ..............................................................

Figura 2.3. Curva tensãox defomaçãodo modelo ............................................................... 8

Figura 2.4. Para solução homogênea do modelo, curvas
(a) Força e
(b) Relação entre taxas de dfomaçãa versus desłocamento.

Figura 2.5. Lei constitutiva para 0 elemento (a) $1 \mathrm{e}$ (b) 2 .........................................10

Figura 2.6. Curva FxU para respostas homogênea e localizadas...............................14

Figura 2.7. D iagrama da variação relativa de deformação em função do deslocamento total U, para (a) localização no elemento 1 e b) localização no elemento 2 ..

Figura 2.8. Barra sujeita a carregamento uniaxial.

Figura 2.9. Curva $\sigma \mathrm{x \varepsilon}$ durante a análise em elementos finitos para $\mathrm{n}=3$ :

(a) descarregamento dos elementos 1 e 3 ;

(b) carregamento do elemento 2 . 16

Figura 2.10. Curva Tensão - D eformação média.

Figura 2.11. (a) Espécime analisado.

(b) Carregamento dinâmico. 17

Figura 2.12. Malhas deformadas ao final da análise. .18 
Figura 2.13. Perfil de dano para as malhas (a) 1, (b) 2, (c) 3 e (d) 4 ...........................18

Figura 2.14. Componentes de tensão e pares de momento para 0 contínuodeCosserat.

Figura 2.15. $\mathrm{G}_{\mathrm{f}}=(\Delta \mathrm{W})+(\Delta \mathrm{U})$, para 0 caso de satteninglinear. .................................22

\section{Capítulo 3}

Figura 3.1. (a) Propagação do fronte de uma onda.

(b) $\mathrm{Em} \mathrm{t}_{\mathrm{n}} \rightarrow \infty$, a onda pode ser considerada como plana. 26

Figura 3.2.a) Barra analisada, com deslocamento imposto ut nas extremidades livres,

b)Lei constitutiva com carregamento negativo.

Figura 3.3. Região plástica $\left(\Omega^{\mathrm{s}}\right)$, elástica $\left(\Omega^{\mathrm{e}}\right)$ e a interface entre as regiões...............30

Figura 3.4. Spectrum de freqüência para modelo de plasticidade linear com gradientes segundo e quarto.

Figura 3.5. Curva de dispersão

Figura 3.6.a) Barra analisada, b)lei constitutiva com encruamento negativo............36

Figura 3.7. Equação 3.47(y) em função da largura de banda s(x)...............................39

Figura 3.8. D istribuição de deformação plástica ao longo da barra.

Figura 3.9. D istribuição de deformação plástica ao longo da barra, para largura de banda wrelativas à (a) segunda ou

(b) quarta raíz da equação 3.49 .

Figura 3.10. Influência de $\mathrm{g}$ quando

(a) $G_{2}=0$ e quando (b) $G_{2}$ é constante e igual a 100.

Influência de $\mathrm{G}_{2}$ quando

$(d) \mathrm{G}=0$ e quando (d) G é constante e igual a 100 .

Figura 3.11. Valores de $\mathrm{W} / \mathrm{G}$ para (a) $\mathrm{G}_{2}=0$, (b) $\mathrm{G}_{2}>0$, (c) $\mathrm{G}_{2}<0$. . .45

\section{Capítulo 4}

Figura 4.1. Modelo de dano clássico unidimensional.

Figura 4.2 (a) Projeção do modelo na superfície de Watergaard;

(b) Comportamento dissimétrico em tração e compressão permitido pelo modelo

Figura 4.3. G ráfico g(q) x qpara: (a) $\mathrm{G}<0$, (b) G $=0$, e (c) G $>0$. .53

Figura 4.4. Resposta tensão- defomaçãouniaxial e pontos de localização para diferentes valores dos parâmetros de difusão. $\mathrm{E}=20000 \mathrm{MPa}$, $\mathrm{b}=.0018, \mathrm{k}=65 \mathrm{~Pa}$. 
Figura 4.5.(a) Curva qx $\mathrm{D}_{\mathrm{\alpha}} \mathrm{e}$ (b) Zoomna curva (a)...................................................... 55

Figura 4.6. Velocidade de fase para modelos de dano A, B e C. ................................ 58

Figura 4.7. Comprimento de onda crítico para modelo de gradiente de quarta e segunda ordem.

\section{Capítulo 5}

Figura 5.1. Esquema das fases de previsão e correção.............................................. 67

Figura 5.2. Matrizes e vetores do elemento TRU-2..................................................... 71

Figura 5.3. Funções de forma, vetores e matrizes da fase de previsão para o elemento CST-3.

Figura 5.4. Elemento de viga: variáveis generalizadas, modelo de dano com gradiente de quarta ordem. 78

\section{Capítulo 6}

Figura 6.1. Exemplo unidimensional: dados geométricos, de carregamento e do material.

Figura 6.2. Evolução da reação no engaste para as três malhas.

Figura 6.3. Curva RauD x tpara o modelo de dano não - local.

Figura 6.4. Perfil de dano em $t=2 \times 10^{4}$, para (a) modelo A e (b) modelo $\mathrm{C}$ de dano, para três valores diferentes dos termos de difusão

Figura 6.5. D issipação de energia no tempo para os modelos local, A e B.

Figura 6.6. D ano no final das análises e a evolução do dano para os modelos $\mathrm{A}(\mathrm{a}, \mathrm{d}), \mathrm{B}(\mathrm{b}, \mathrm{e})$ e $\mathrm{C}(\mathrm{c}, \mathrm{f})$, respectivamente.

Figura 6.7. (a) Evolução do dano para modelo A.

(b) Evolução do dano para modelo B

Figura 6.8. (a) Características geométricas,

(b) dados do carregamento e (c) características do material 85

Figura 6.9. D eslocamento no tempo para modelos

(a) de dano local e modelo B e (b) modelos B e A 86

Figura 6.10. Configuração deformada para:

(a) modelo local, (b) modelo A, (c) modelo B.

Figura 6.11. D ados geométricos do espécime;

Malhas com (b) 516 e (c) 1230 elementos. 88

Figura 6.12. Força aplicada versus abertura do entalhe. 89

Figura 6.13. Evolução da deformação, malha de 516 elementos e aberturas de (a)0.1, (b)0.18, (c)0.26 e (d)0.34 mm. 89 
Figura 6.14. Evolução da deformação para malha de 1230 elementos e aberturas: (a)0.1,(b)0.18,(c)0.26 e (d)0.34 mm.

Figura 6.15. Evolução do dano para abertura de:

(a) $0.1,(\mathrm{~b}) 0.18,(\mathrm{c}) 0.26$ e (d) $0.34 \mathrm{~mm}$

Figura 6.16. G eometria da viga e parâmetros do material.

Figura 6. 17. Malhas usadas na análise da estrutura: (a) 724 (malha 1),

(b) 1412 (malha2) e (c) 2148 (malha 3) elementos.

Figura 6.18. Curva Força (P) - D eflexão (u).

Figura 6.19. Configuração da deformada com deslocamento central imposto de $0.5 \mathrm{~mm}$, para as malhas (a) 1, (b) 2 e (c) 3 92

Figura 6.20. Evolução da variável dano (malha 2)..... 


\section{Lista de Quadros}

\section{Capítulo 5}

Q uadro 5.1. Matrizes e vetores de variáveis generalizadas do elemento TRU-2, para 0 caso $b_{1} b_{2} \neq 0$............................................................................... 72

Q uadro 5.2. Matrizes e vetores de variáveis generalizadas do elemento TRU-2,

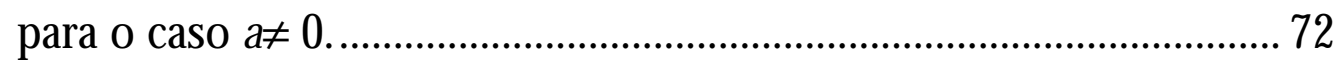

Q uadro 5.3. Vetores e matrizes de variáveis generalizadas do elemento CST-3, para o caso $b_{1} b_{2} \neq 0$. 75

Q uadro 5.4. Vetores e matrizes de variáveis generalizadas do elemento CST-3, para o caso $a \neq 0$. 75

Q uadro 5.5. Seqüência de cálculo para obter o vetor $\mathrm{G}$ 79 
Lista de tabelas

\section{Capítulo 2}

Tabela 2.1. Valores para estudo numérico do item 2.2........................................... 14

\section{Capítulo 5}

Tabela 5.1. Elementos finitos implementados.

.68 


\section{Lista de símbolos}

a

$\mathrm{b}_{1} \mathrm{i}=1, . ., 3$

C

C

$\mathrm{C}_{\mathrm{e}}$

$\mathrm{C}_{\mathrm{f}}$

$\mathrm{G}_{1}, \mathrm{C}_{2}$

D

E

$E_{\text {sec }}$

$\mathrm{f}$

$g_{1}, g_{2}$

G

$\mathrm{G}_{\mathrm{f}}$

$\mathrm{h}$

$\mathrm{k}$

L

$L_{p}$

$L_{c}, L_{c}^{*}$

$\ell_{1}, \ell_{2}$ parâmetro adimensional $[0,1]$ do modelo de dano não local parâmetros do material para o modelo de dano apresentado no Capítulo 4 (parâmetro bs é chamado genericamente de bem algumas situações)

matriz de compatibilidade entre deformações e deslocamentos velocidade de propagação do fronte de onda velocidade de propagação elástica velocidade de fase

parâmetros de difusão: G acompanha o gradiente segundo

$\mathrm{C}_{2}$ acompanha 0 gradiente quarto

variável de dano

tensor constitutivo de rigidez elástica

tensor constitutivo de rigidez secante

função de escoamento (plástico ou de dano)

representação de ondas de forma arbitrária

módulo elástico de cisalhamento

energia de fratura

inclinação da curva softeningpara modelos elasto-plásticos

parâmetro do material para o modelo de dano apresentado no

Capítulo 4

comprimento da barra unidimensional

funcional da fase de previsão

funcional da fase de correção

parâmetros internos do material (comprimentos internos) 


\begin{tabular}{ll}
$\mathrm{m}$ & direção do deslocamento de um ponto \\
$\mathrm{N}$. & vetor de forma da variável $(\bullet)$. \\
$\mathrm{n}$ & normal à superfície de plastificação ou danificação $\mathrm{f}$ \\
$\mathrm{n}$ & direção de propagação da onda \\
$\mathrm{q}$ & número de onda \\
$\mathrm{S}$ & fase da função de onda; largura da zona de localização \\
$\mathrm{t}$ & tempo \\
$\mathrm{u}$ & deslocamentos \\
$\mathrm{v}$ & velocidade \\
$\mathrm{X}$ & coordenada \\
$\mathrm{Y}$ & taxa de dissipação \\
$\alpha, \beta$ & constantes utilizadas no item 2.2 \\
$\Delta t$ & passo finito de tempo \\
$\Delta(\bullet)$ & Laplaciano de $(\bullet)$ \\
$\varepsilon$ & deformações \\
$\bar{\varepsilon}$ & deformação máxima \\
$\widehat{\varepsilon}$ & deformação média \\
$\varepsilon^{\mathrm{e}}$ & deformação elástica \\
$\varepsilon^{\mathrm{p}}$ & deformação plástica \\
$\widetilde{\varepsilon}$ & deformação efetiva \\
$\phi$ & função de onda simples \\
$\dot{\lambda}$ & um escalar não negativo representativo da intensidade da taxa \\
$\lambda$ & de deformação plástica \\
$\lambda$ & comprimento de onda \\
$\nu$ & comprimento de onda crítico \\
$\rho$ & coeficiente de Poisson \\
$\rho$ & densidade \\
$\sigma$ & tensões \\
$\bar{\sigma}$ & tensão máxima \\
$\widetilde{\sigma}$ & tensão efetiva \\
$\sigma^{\mathrm{d}}$ & tensão equivalente \\
$\tau$ & inclinação da banda de localização \\
$\omega$ & energuência livre de Helmhotz \\
& \\
\hline &
\end{tabular}




\section{RESUMO}

O plano de trabalho proposto insere-se no âmbito da linha de pesquisas em Mecânica de Materiais com aplicação da Mecânica do dano Contínuo, dando-se ênfase para a questão de localização de deformações decorrente do emprego de modelos constitutivos com encruamento negativo (softening). Em particular, a motivação é oferecer uma contribuição ao estudo de uma das técnicas de regularização da resposta numérica, que objetivam eliminar a dependência dos resultados com relação à discretização adotada. Tal técnica de regularização consiste na consideração de gradientes da variável de dano na formulação dos modelos constitutivos. Tanto aspectos teóricos como de aplicação numérica são explorados, destacando-se nos primeiros uma análise do efeito da inclusão de gradientes de ordem superior na relação constitutiva e o emprego do Método dos Elementos Finitos em sua formulação em variáveis generalizadas no segundo aspecto. Outros aspectos complementares são também discutidos, relacionados à formulação variacional e algoritmos de integração em passo finito dos modelos em estudo. Os exemplos de aplicação consistem de análises estática e dinâmica de estruturas, realizando-se um confronto com resultados disponíveis na literatura ou mesmo com observações experimentais de ensaios de laboratório. 
This work is inserted in the researches in Material Mechanics with an application in Continuum Damage Mechanics, emphasising the issue about localisation of deformations according to the use of constitutive models with softening. In particular, the motivation is to offer a contribution to one of the regularisation techniques of the numerical response, aiming to eliminate mesh dependence of the results. This regularisation technique consists in considering gradients of the damage variable in the formulation of constitutive models. Theoretical aspects as well as numerical application are explored, highlighting the analysis of the effect of the inclusion of gradients in the constitutive relation in the first aspect, and using Finite Element Methods with generalise variables in the second one. Other complementary aspects will also be discussed associated to the variational formulation and integration algorithms in finite steps of the studied models. The examples consist in dynamic and static analysis of structures, comparing with numerical and experimental results available in the literature. 


\subsection{Aspectos gerais e objetivos do trabalho}

Fissuração progressiva em materiais quase - frágeis como o concreto resulta em perda de resistência e rigidez. No contexto da mecânica do contínuo, a representação gráfica da relação tensão - deformação, exibe um ramo sattening ou de encruamento negativo, devido à diminuição de resistência. Nesse ramo, a rigidez tangente do material deixa de ser positiva definida e a rigidez secante diminui progressivamente, também devido à danificação, podendo-se incorporar ou não deformações permanentes. Os trabalhos pioneiros de Rice ${ }^{1}$, Maier \& Huekkd ${ }^{2}$, tratam dos problemas da formulação de modelos constitutivos com satteninge do mal posicionamento das equações governantes.

Com uso de modelos constitutivos com encruamento negativo em uma análise numérica em elementos finitos, os resultados dependem da discretização adotada. 0 estudo da localização de deformações em combinação com tais tipos de modelos constitutivos, permite esclarecer a razão desta não objetividade (Benallal $\notin$ al. ${ }^{3}$, Rizzi ${ }^{4}$ ). De fato, os modelos locais incluem, em resultados numéricos, uma resposta não homogênea que converge para uma descontinuidade com dissipação nula de energia.

Uma maneira de contornar este problema é formular o modelo constitutivo como não local (Bazant \& al. ${ }^{5}$, Pijauder-Cabot \& Bazant ${ }^{6}$ ) de onde derivam as chamadas técnicas de regularização. Em última análise, estes modelos restringem a zona de localização à uma zona de largura finita, o que permite recuperar a objetividade. 
Entre as várias técnicas de regularização da resposta numérica existentes na literatura, este trabalho trata daquela que consiste na introdução, no modelo constitutivo, de gradientes de ordens superiores de variáveis relacionadas à plasticidade (Mühlhaus \& Aifantis ${ }^{7}$, Slyus $^{8}$ ) ou dano - Frémond \& Neejar ${ }^{9}$, Com $^{10}$, Comi \& Driemier ${ }^{11}$.

A principal contribuição do trabalho está no estudo e implementação de um modelo de dano com gradientes de segunda e quarta ordem.

\subsection{O conteúdo dos capítulos da tese}

No segundo capítulo discute-se o fenômeno da localização e mostra-se que os modelos constitutivos com sattering incluem a resposta localizada, além daquela homogênea. Essas características são ilustradas no exemplo sugerido por Billardon ${ }^{12}$ e reproduzido neste capítulo. Os problemas relacionados à dependência de malha são ilustrados através de dois exemplos clássicos envolvendo modelos de plasticidade (Slyus ${ }^{8}$, Sluys \& al. ${ }^{13}$, Pamim ${ }^{14}$, Com \& Peeeg ${ }^{15}$ ). Em seguida, são revistos, brevemente, os métodos de regularização propostos na bibliografia e que pretendem oferecer alternativa para a recuperação da objetividade das respostas numéricas.

O terceiro capítulo apresenta o método de regularização com gradiente em modelos elastoplásticos. A analogia entre o problema de localização e 0 da propagação de ondas permite interpretar as principais características do modelo proposto. Neste particular, este trabalho estende a análise proposta por Slyus ${ }^{8}$, incluindo-se o gradiente de quarta ordem. Um exemplo analítico em campo unidimensional, ilustra 0 estudo desenvolvido. Além disso, evidenciam-se algumas características já ressaltadas por Z Zib \& Aifantis ${ }^{16}$, Mïhlhaus \& Aifantis ${ }^{7}$, como a possibilidade de localização com encruamento positivo (hardening.

O quarto capítulo apresenta inicialmente a forma local do modelo de dano sugerido em Comi \&al. ${ }^{17}$. Em seguida, discute-se uma versão não - local do mesmo modelo, com a introdução dos gradientes de segunda e quarta ordem na função critério de danificação, incorporando-se, ainda, um parâmetro de difusão não constante e dependente do nível de dano. Desta maneira, três formas particulares do modelo são definidas. Um estudo analítico unidimensional mostra que a consideração do gradiente de quarta ordem permite identificar situações de dano crítico (para início de localização) que podem ocorrer antes do pico de tensão. Conclui-se o capítulo com um estudo de propagação de ondas, que proporciona uma análise sobre a largura da zona de localização. 
No quinto capítulo, trata-se da discretização no tempo e no espaço do modelo de dano. No processo iterativo de integração do modelo constitutivo, utilizam-se uma formulação variacional para fase elástica de previsão e outra para fase não-linear de correção. A utilização de dois funcionais distintos é discutida em $\mathrm{Com}^{10}$ e Balbo ${ }^{18}$. Para cada tipo particular de elemento utilizado, apresentam-se os funcionais discretizados em variáveis generalizadas (Comi $\mathbb{a}$ al. ${ }^{19}$ ), as funções de forma, e as equações governantes dos problemas de previsão e correção.

O último capítulo reúne exemplos de aplicação do modelo de dano com gradiente, em análises estática e dinâmica. Os resultados numéricos indicam que a consideração de um parâmetro de difusão não constante leva a uma zona de localização de largura finita. 


\section{FENÔMENO DE LOCALIZACÃO}

\subsection{Apresentação do fenômeno de localização}

Muitos materiais empregados na engenharia, dúcteis e quasi-frágeis, apresentam resposta caracterizada por um regime elástico bem definido, onde todos os processos são considerados reversíveis, e um regime não elástico, resultado da manifestação de fenômenos irreversíveis que precedem a ruptura. Neste último, normalmente identificam-se os encruamentos positivo (hardaning e negativo (sottering, sendo que 0 encruamento negativo é conseqüência do aparecimento de pronunciados gradientes de deslocamento em zonas restritas do meio, chamadas zonas de loalização de defomaçãa $O$ fenômeno da localização, por sua vez, pode ser induzido pela geometria do corpo, condições de contorno ou heterogeneidade e defeitos locais do material.

As zonas de localização foram primeiramente observadas em ensaios experimentais de tração de corpos de prova metálicos. Neste caso, as deformações plásticas acabam por se concentrar, em nível macroscópico, na forma de bandas dedisalhamentoou bandas de Lüdar. Na literatura, estão disponíveis muitas publicações sobre a observação experimental da localização de deformações em metais - Nadai ${ }^{20}$, Asaro ${ }^{21}$, Perzyna \& Pezzyna ${ }^{22}$ eOlmsteded al. ${ }^{23}$.

No caso de materiais como o concreto, o principal fenômeno dissipativo é a micro fissuração, cuja evolução decorrente do aumento dos níveis de solicitação mecânica, por exemplo, tende a concentrar-se em uma zona de largura muito pequena. A justaposição de microfissuras contidas nessa zona acaba por levar à formação de uma macrofissura, conforme Ceedlin $\notin$ al. ${ }^{24}$. A largura e direção da zona de localização dependem do tipo de concreto e dos defeitos nele contidos, da geometria, condições 
de contorno e carregamento (Pamin ${ }^{14}$ ). A Figura 2.1 ilustra a evolução da fissuração no concreto.

$\mathrm{Na}$ modelagem matemática de estruturas em concreto, pode-se tratar cada macrofissura individualmente, como sugere o método da fratura discreta, ou modelar o processo de evolução da microfissuração inteiramente via lei constitutiva local, válida ponto a ponto, como sugerem os modelos de meios contínuos equivalentes, como os da mecânica do dano.

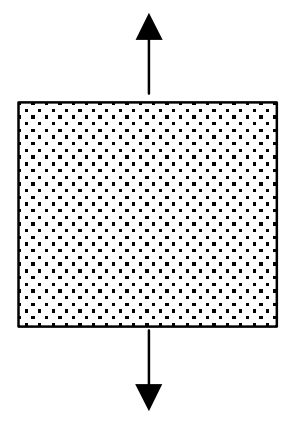

(a)

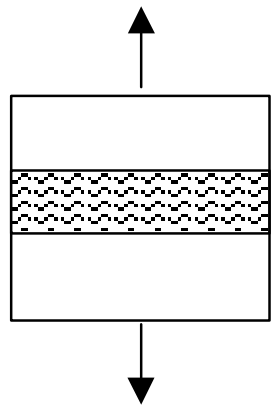

(b)

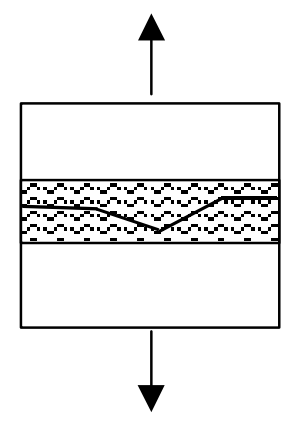

(c)

Figura 2.1. - (a) Fissuração em estágio inicial de solicitação: distribuída ao longo da estrutura;

(b) Localização do fenômeno de fissuração; (c) Formação da macrofissura.

Apesar de todos os méritos de cada método, não se pode, por exemplo, em uma estrutura de grande escala, modelar cada fissura individualmente, como sugere 0 método da fratura discreta. Esse método também pressupõe a existência inicial da macrofissura, desconsiderando todo 0 processo anterior de evolução da microfissuração. Por outro lado, para modelar a localização de deformação em combinação com os modelos contínuos, o maior problema que se apresenta é o da identificação e orientação da zona de localização. Além disso, alguma forma de adaptação de malha deve ser adotada para que se possa inserir dentro da zona elementos com lei de encruamento negativo bem definida.

Mas aí uma outra questão surge; supondo que tenha identificada uma região de localização, qual a largura da zona?

Nos modelos constitutivos clássicos, baseados em uma descrição local do comportamento do material, a largura da zona de localização tende a zero, uma vez que a simulação procura encontrar uma condição compatível com uma fratura discreta. Basta pensar, por simplicidade, em elementos com deformação constante para os quais necessariamente a largura da zona de localização fica associada às suas dimensões. Com o refinamento da malha, a largura da zona tende a zero, obtendo-se soluções que convergem para uma resposta de energia dissipada nula. Matematicamente, diz-se que o problema de valor de contorno resulta mal 
posicionado (no sentido de existência de um número finito de soluções linearmente independentes que dependem continuamente dos dados). $\mathrm{O}$ mal posicionamento, nestes casos, ocorre com perda de elipticidade em análise estática e perda de hiperbolicidade em análise dinâmica, ou quando as condições complementares de contorno e interface não se correspondem (Benallal \& al. ${ }^{3}$ ). Neste caso, a descrição matemática deixa de ser uma representação coerente da realidade física.

É possível, entretanto, empregar, na formulação contínua, técnicas que não necessariamente sofrem de perda de elipticidade do campo de equações quando a zona de localização de deformações se desenvolve estando, portanto, capacitadas à prover soluções numéricas representativas dos problemas de valor de contorno envolvendo encruamento negativo e localização.

Uma técnica bastante simples de regularização seria impedir que numericamente a zona localizada tendesse a uma linha, impondo-se a ela uma largura mínima e encontrando-se a condição de ruptura ou formação de uma fratura discreta, quando 0 estado de deformação dentro dela atingisse um valor limite. Para que a fixação de uma largura não pareça ser um recurso não muito bem fundamentado, uma justificativa que se encontra na literatura é imaginar que ela seja uma 'propriedade' do material.

Mas uma maneira mais elegante e ao mesmo tempo matematicamente correta de implementar esta idéia consiste em formular o problema de modo que a largura da zona esteja implícita no modelo resultante. Essa maneira é proporcionada pelos chamados modelos não-locais como os modelos com introdução de gradientes de ordem superior da deformação plástica ou da variável de dano na função de escoamento ou de danificação - Frémond \& Neđjar ${ }^{9}$, Peedings \& al. ${ }^{25}$, DeBarst \& al. ${ }^{26}$, $\mathrm{Comi}^{10,27}$, Comi \& Driemier ${ }^{11,28}$. Este trabalho trata exatamente desse tipo de modelo. Entre outras técnicas de regularização existentes destacam-se: teoria micropolar - de Barst \& Muhlhaus ${ }^{29}$, Steinmam \& Stein ${ }^{30}$, Iordache \& Willam ${ }^{31}$, integral não-local Pijaudier-Cabot \& Bazant ${ }^{5}$, energia de fratura - Oliver ${ }^{32}$.

Resta outra questão importante: onde se inicia a localização?

Em muitos problemas a identificação da região de localização pode ser diretamente decorrente da distribuição de esforços (naturalmente nas zonas mais solicitadas) e de descontinuidades geométricas. Porém quando ela depende da distribuição inicial de defeitos do material, a qual possui aleatoriedade, a resposta torna-se mais difícil de ser prevista. $\mathrm{O}$ problema torna-se ainda mais difícil quando se combina à distribuição de defeitos os efeitos de condições de vinculação (pode ser observada no caso simples de tração uniaxial). 
Deixando-se de lado o efeito dos vínculos de contorno, a localização por defeitos se dá nas partes menos resistentes do material. Mas aí a simulação numérica deve recorrer a funções de distribuição as quais permitem identificar os pontos mais frágeis do meio. Neste trabalho não se fará uso de tais funções, uma vez que o objetivo principal é estudar uma técnica de regularização; quando necessário opta-se por prowara a localização pela adoção de uma resistência menor em determinados pontos da estrutura.

No que segue, apresenta-se exemplos que ilustram a questão da não - objetividade de resposta dos modelos locais e discute-se, sucintamente, outras técnicas de regularização.

\subsection{Exemplo ilustrativo do fenômeno na análise numérica}

Com o objetivo de ilustrar o fenômeno da localização, será analisada uma barra uniaxial (Figura 2.2), por meio de um modelo de dano, para os casos de respostas homogênea e localizada. Este estudo foi originalmente realizado em Billardan ${ }^{12}$.

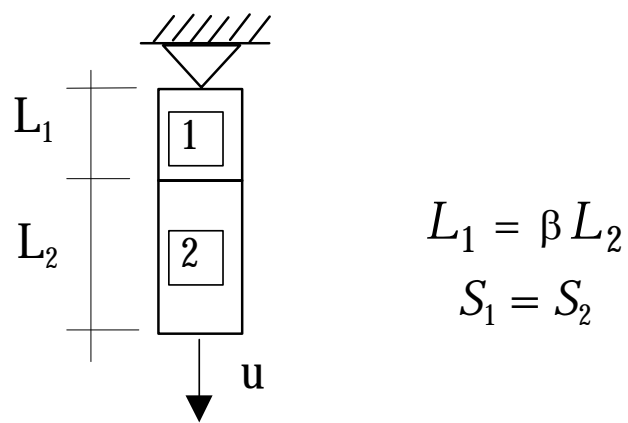

Figura 2.2. Dados geométricos da barra.

A Figura 2.3 ilustra a lei constitutiva do material, que é dada pela clássica relação:

$\sigma=\mathrm{E}(1-\mathrm{D}) \varepsilon$

D é a variável representativa do grau de danificação e varia entre $[0,1]$, onde $D=0$ representa 0 material sem dano, enquanto $\mathrm{D}=1$ representa a fratura do material. A lei de evolução da variável de dano é dada por:

$\dot{\mathrm{D}}=\mathrm{k} \dot{\varepsilon}$

onde ké uma constante do material. 
As equações de equilíbrio e compatibilidade para o problema são:

$\sigma_{1} \mathrm{~S}_{1}-\sigma_{2} \mathrm{~S}_{2}=0$

$\varepsilon_{1} \mathrm{~L}_{1}+\varepsilon_{2} \mathrm{~L}_{2}=\mathrm{U}$

onde U é o deslocamento imposto na estrutura.

Da equação 2.3a tem-se, em qualquer instante, $\sigma_{1}=\sigma_{2}$, uma vez que $\mathrm{S}_{1}=\mathrm{S}_{2}$. Portanto, igualando-se o valor da tensão máxima $\left(\sigma_{\max }=\mathrm{E} / 4 \mathrm{k}\right)$ nos dois elementos, segue que:

$$
\frac{\mathrm{E}_{01}}{\mathrm{E}_{02}}=\frac{\mathrm{k}_{1}}{\mathrm{k}_{2}}=\alpha
$$

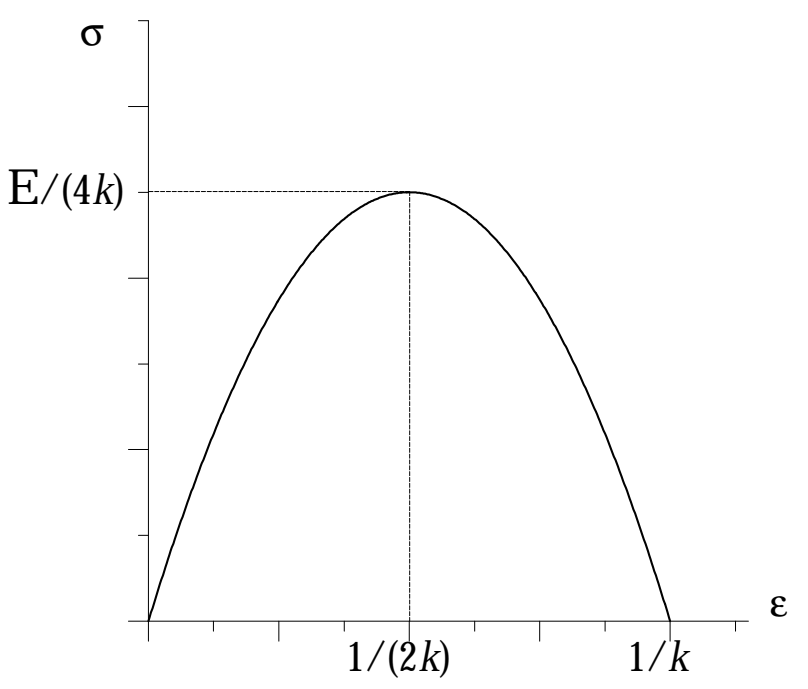

Figura 2.3. Curva tensão x deformação do modelo.

$\mathrm{Ou}$, ainda pela condição $\sigma_{1}=\sigma_{2}$, pode-se igualar a lei constitutiva particularizada para cada elemento e substituir na lei de evolução do dano, de modo a obter-se:

$\mathrm{D}_{1}=\mathrm{D}_{2}=\mathrm{k}_{1} \varepsilon_{1}=\mathrm{k}_{2} \varepsilon_{2} \quad \forall \varepsilon_{1}, \varepsilon_{2}$

para carregamento monótono crescente.

A estrutura pode apresentar os comportamentos: homogêneo, localização de dano no elemento 1, localização de dano no elemento 2 . A seguir, estuda-se com detalhes cada um destes comportamentos. 


\subsubsection{Solução homogênea}

Considerando-se as equações 2.4 e 2.5, a solução do sistema 2.3 é do tipo:

$\varepsilon_{1}=\frac{\mathrm{U}}{(\alpha+\beta) \mathrm{L}_{2}} \quad$ e $\quad \varepsilon_{2}=\frac{\alpha \mathrm{U}}{(\alpha+\beta) \mathrm{L}_{2}}$

Portanto:

$\mathrm{F}=\sigma \mathrm{S}=\left(\frac{\mathrm{E}_{01}}{(\alpha+\beta) \mathrm{L}_{2}} \mathrm{U}-\frac{\mathrm{E}_{01} \mathrm{k}_{1}}{(\alpha+\beta)^{2} \mathrm{~L}_{2}^{2}} \mathrm{U}^{2}\right) \mathrm{S}$

com uma taxa de deformação:

$\dot{\varepsilon}_{1}=\frac{\mathrm{U}}{(\alpha+\beta) \mathrm{L}_{2}} ; \dot{\varepsilon}_{2}=\frac{\alpha \mathrm{U}}{(\alpha+\beta) \mathrm{L}_{2}} \quad \therefore \frac{\dot{\varepsilon_{1}}}{\dot{\varepsilon_{2}}}=\frac{1}{\alpha}$

A Figura 2.4 ilustra as variações de $\mathrm{F}$ e $\dot{\varepsilon_{1}} / \dot{\varepsilon_{2}}$ com o deslocamento U. Para 0 caso homogêneo, $\dot{\varepsilon_{1}} / \dot{\varepsilon_{2}}$ é constante até a ruptura $(\mathrm{D}=1)$. Isto significa que $\varepsilon_{1}$ e $\varepsilon_{2}$ crescem proporcionalmente durante toda a história de carregamento: $\varepsilon_{1}=\alpha \varepsilon_{2}$. Não há localização de deformações em nenhum dos elementos.

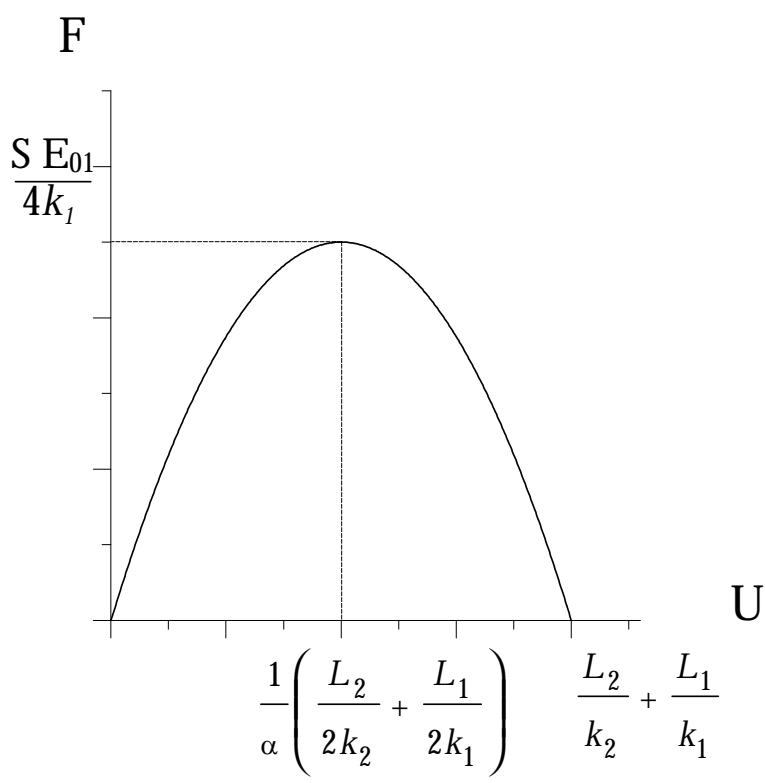

(a)

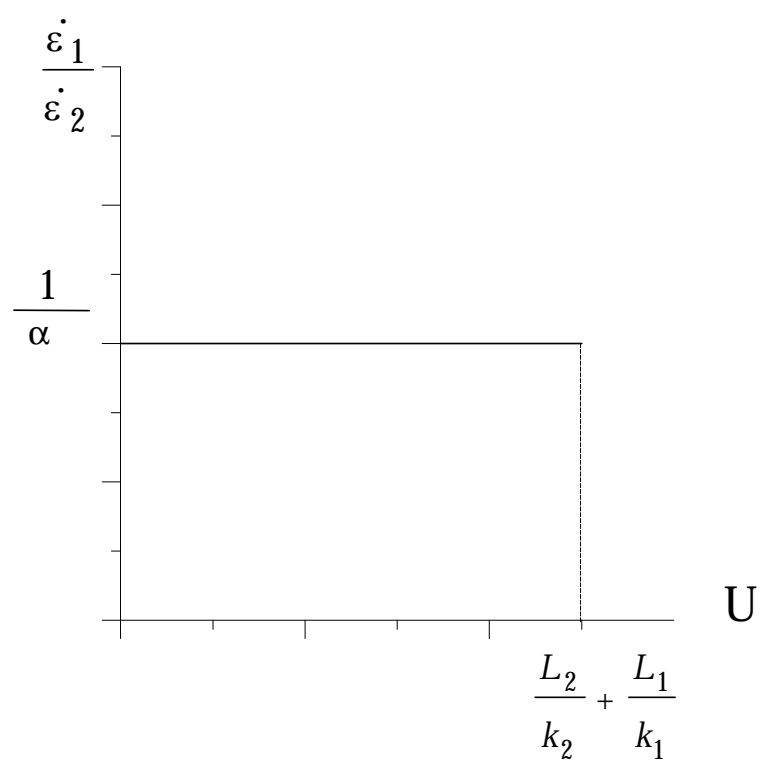

(b)

Figura 2.4. Para solução homogênea do modelo, curvas (a) Força e (b) Relação entre taxas de deformação, versus deslocamento. 


\subsubsection{Resposta localizada em 2}

A partir do valor de deslocamento que provoca tensão máxima em um dos elementos, há a possibilidade de descarregamento no outro elemento. Neste caso, supondo que o elemento 1 sofre descarga elástica com valor de dano constante $\widehat{D}_{1}$ e o elemento 2 carga com evolução de dano, o sistema 2.3 passa a ser escrito na forma:

$$
\begin{aligned}
& \mathrm{E}_{01}\left(1-\mathrm{D}_{1}\right) \varepsilon_{1}-\mathrm{E}_{02}\left(1-\mathrm{k}_{2} \varepsilon_{2}\right) \varepsilon_{2}=0 \\
& \mathrm{~L}_{1} \varepsilon_{1}+\mathrm{L}_{2} \varepsilon_{2}=\mathrm{U}
\end{aligned}
$$

A Figura 2.5 ilustra a lei constitutiva de cada elemento. Q uando o elemento 2 atinge 0 valor máximo de tensão, este inicia 0 ramo de encruamento negativo, provocando descarga elástica no elemento 1, que volta à origem, uma vez que 0 modelo de dano não admite deformação plástica.

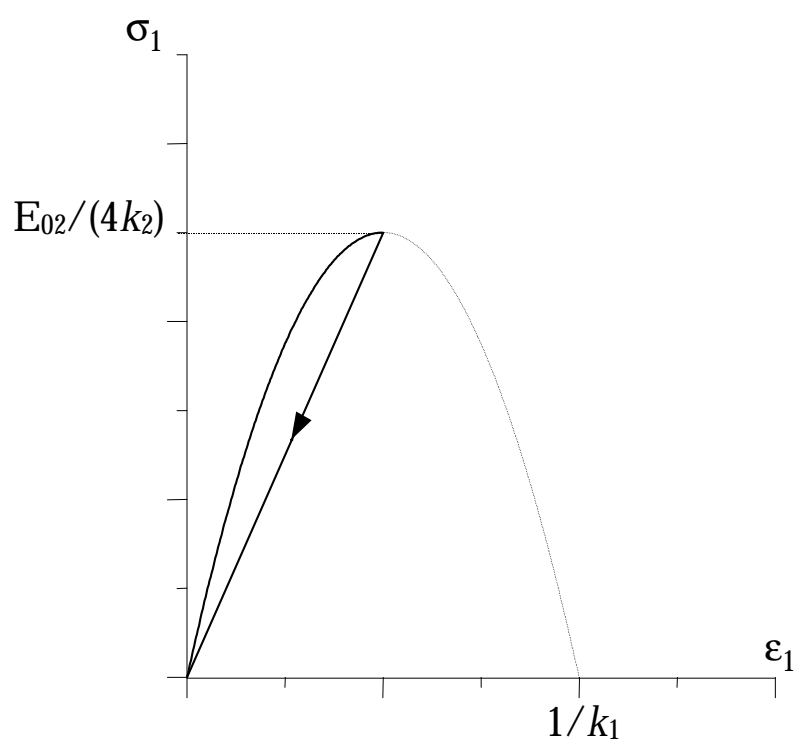

(a)

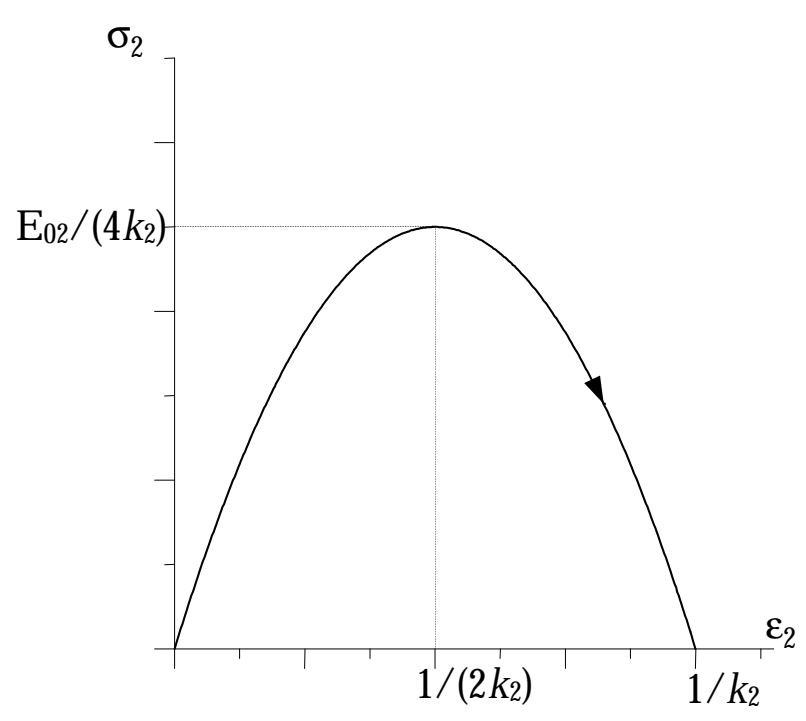

(b)

Figura 2.5. Lei constitutiva para o elemento (a) 1 e (b) 2.

Da equação 2.9:

$\alpha\left(1-\mathrm{D}_{1}\right) \varepsilon_{1}-\left(1-\mathrm{k}_{2} \varepsilon_{2}\right) \varepsilon_{2}=0$

ou, em termos de taxas:

$\alpha\left(1-\widehat{D}_{1}\right) \dot{\varepsilon_{1}}-\left(1-2 \mathrm{k}_{2} \varepsilon_{2}\right) \dot{\varepsilon_{2}}=0$ 
Da equação anterior:

$\frac{\dot{\varepsilon_{1}}}{\dot{\varepsilon_{2}}}-=\frac{\left(1-2 \mathrm{k}_{2} \varepsilon_{2}\right)}{\alpha\left(1-\overline{\mathrm{D}}_{1}\right)}$

Por outro lado, de 2.10:

$\varepsilon_{1}=\frac{\mathrm{U}}{\mathrm{L}_{1}}-\frac{\varepsilon_{2}}{\beta}$

que substituída em 2.9 conduz a:

$\mathrm{U}=\mathrm{L}_{1}\left[\frac{1}{\beta}+\frac{1}{\alpha\left(1-\overline{\mathrm{D}}_{1}\right)}\right] \varepsilon_{2}-\frac{\mathrm{k}_{2} \mathrm{~L}_{1}}{\alpha\left(1-\overline{\mathrm{D}}_{1}\right)} \varepsilon_{2}^{2}$

Derivando a equação anterior:

$\frac{\mathrm{dU}}{\mathrm{d}_{2}}=\mathrm{L}_{1}\left[\frac{1}{\beta}+\frac{1}{\alpha\left(1-\mathrm{D}_{1}\right)}\right]-\frac{2 \mathrm{k}_{2} \mathrm{~L}_{1}}{\alpha\left(1-\mathrm{D}_{1}\right)}$

obtém-se os valores máximos de $\varepsilon_{2}$ e U:

$\varepsilon_{2 \max }=\frac{\alpha\left(1-\hat{\mathrm{D}}_{1}\right)}{2 \mathrm{k}_{2}}\left[\frac{1}{\beta}+\frac{1}{\alpha\left(1-\hat{\mathrm{D}}_{1}\right)}\right]$

$\mathrm{U}_{\max }=\frac{\mathrm{L}_{1}}{4 \mathrm{k}_{2}} \alpha\left(1-\mathrm{D}_{1}\right)\left[\frac{1}{\beta}+\frac{1}{\alpha\left(1-\mathrm{D}_{1}\right)}\right]^{2}$

E $\varepsilon_{1}$ para os valores de $U_{\text {max }}$ e $\varepsilon_{2 \text { max }}$ correspondentes:

$\varepsilon_{1 \text { max }}=\frac{\alpha\left(1-\hat{D}_{1}\right)}{2 \mathrm{k}_{2}}\left\lfloor\frac{1}{\alpha^{2}\left(1-\hat{D}_{1}\right)^{2}}-\frac{1}{\beta^{2}}\right\rfloor$

Portanto, o snap-badk na resposta global se inicia para o valor de $U_{\max }$ que corresponde às deformações $\varepsilon_{1 \max }$ e $\varepsilon_{2 \max }$ dos elementos 1 e 2 , respectivamente. 
Pode-se pesquisar a relação $\dot{\varepsilon_{1}} / \dot{\varepsilon_{2}}$ nos casos:

$\varepsilon_{2}=\frac{1}{2 \mathrm{k}_{2}} \quad$ e $\quad \varepsilon_{2 \max }=\frac{\alpha\left(1-\hat{\mathrm{D}}_{1}\right)}{2 \mathrm{k}_{2}}\left\lceil\frac{1}{\beta}+\frac{1}{\alpha\left(1-\hat{\mathrm{D}}_{1}\right)}\right\rfloor$

Substituindo em 2.13 as equações 2.20a e b, tem-se, respectivamente:

$$
\frac{\dot{\varepsilon}_{1}}{\dot{\dot{\varepsilon}_{2}}}=0 \quad \frac{\dot{\varepsilon}_{1}}{\dot{\varepsilon}_{2}}=-\frac{1}{\beta}
$$

Para que $\mathrm{U}_{\max }$ ocorra dentro dos limites admissíveis de $\varepsilon_{2}$, a seguinte condição deve valer:

$\varepsilon_{2} \leq \frac{1}{k_{2}}$

ou ainda, substituindo-se a equação 2.20b na equação anterior:

$\frac{\alpha}{\beta} \leq \frac{1}{\left(1-\mathrm{D}_{1}\right)}$

\subsubsection{Solução localizada em 1}

Neste caso, 0 elemento 1 atinge 0 valor máximo de tensão e inicia 0 ramo de encruamento negativo com acréscimo de dano, enquanto o elemento 2 descarrega com valor de dano constante $\overline{\mathrm{D}}_{2}$. $\mathrm{O}$ sistema (2.1) passa a ser escrito na forma:

$$
\begin{aligned}
& \mathrm{E}_{01}\left(1-\mathrm{k}_{1} \varepsilon_{1}\right) \varepsilon_{1}-\mathrm{E}_{02}\left(1-\overline{\mathrm{D}}_{2}\right) \varepsilon_{2}=0 \\
& \mathrm{~L}_{1} \varepsilon_{1}+\mathrm{L}_{2} \varepsilon_{2}=\mathrm{U}
\end{aligned}
$$

Seguindo de maneira análoga ao item anterior, tem-se:

$$
\begin{aligned}
& \frac{\dot{\varepsilon_{2}}}{\dot{\varepsilon_{1}}}-=\alpha \frac{\left(1-2 \mathrm{k}_{1} \varepsilon_{1}\right)}{\left(1-\mathrm{D}_{2}\right)} \\
& \mathrm{U}=\frac{\mathrm{L}_{2}}{\left(1-\mathrm{D}_{2}\right)}\left\{\left[\left(1-\mathrm{D}_{2}\right) \beta+\alpha\right] \varepsilon_{1}-\alpha \mathrm{k}_{1} \varepsilon_{1}^{2}\right\}
\end{aligned}
$$


D erivando a equação anterior obtém-se os valores máximos de $\varepsilon_{1}$ e U:

$$
\begin{aligned}
& \varepsilon_{1 \text { max }}=\frac{1}{2 \alpha \mathrm{k}_{1}}\left[\left(1-\overline{\mathrm{D}}_{2}\right) \beta+\alpha\right] \\
& \mathrm{U}_{\text {max }}=\frac{\mathrm{L}_{2}}{4 \mathrm{k}_{1}\left(1-\mathrm{D}_{2}\right)}\left[\left(1-\mathrm{D}_{2}\right) \beta+\alpha\right]^{2}
\end{aligned}
$$

E $\varepsilon_{2}$ para os valores de $U_{\max }$ e $\varepsilon_{1 \text { max }}$ correspondentes:

$\varepsilon_{2 \text { max }}=\frac{\alpha}{4 \mathrm{k}_{1}\left(1-\mathrm{D}_{2}\right)}\left[\alpha-\left(1-\mathrm{D}_{2}\right)^{2} \frac{\beta^{2}}{\alpha^{2}}\right]$

de modo que agora pode-se pesquisar a relação $\dot{\varepsilon_{2}} / \dot{\varepsilon_{1}}$ nos casos:

$$
\varepsilon_{1}=\frac{1}{2 \mathrm{k}_{1}} \quad \text { e } \quad \varepsilon_{1_{\text {max }}}=\frac{\alpha+\left(1-\widehat{\mathrm{D}}_{2}\right) \beta}{2 \alpha \mathrm{k}_{1}}
$$

Substituindo em 2.26 as equações 2.31a e b, tem-se, respectivamente:

$$
\frac{\dot{\varepsilon}_{2}}{\dot{\varepsilon_{1}}}=0 \quad \frac{\dot{\varepsilon}_{2}}{\dot{\varepsilon}_{1}}=-\beta
$$

Valendo a seguinte condição:

$$
\frac{\beta}{\alpha} \leq \frac{1}{\left(1-\bar{D}_{2}\right)}
$$

\subsection{Exemplos numéricos}

Primeiramente, ilustra-se o fenômeno da localização dando valores ao estudo feito no item 2.2. Posteriormente, através de dois exemplos, caracterizam-se os problemas numéricos das teorias convencionais que empregam modelos locais com encruamento negativo. 


\subsubsection{Análise do exemplo unidimensional}

A Tabela 2.1 mostra os valores numéricos empregados para a análise do item 2.2.

\begin{tabular}{|l|ll|}
\hline SíMBOLO & VALORES \\
\hline $\mathrm{S}=\mathrm{S}_{1}=\mathrm{S}_{2}$ & 1.0 \\
\hline $\mathrm{k}_{1}$ & 1000 \\
\hline $\mathrm{k}_{2}$ & 2000 \\
\hline $\mathrm{E}_{01}$ & 2000 \\
\hline $\mathrm{E}_{02}$ & 4000 \\
\hline $\mathrm{L}$ & 200 & $\mathrm{~L}_{1}=0.2 \mathrm{~L}=40 \quad \mathrm{~L}_{2}=0.8 \mathrm{~L}=160$ \\
\hline$\alpha=\frac{\mathrm{E}_{01}}{\mathrm{E}_{02}}$ & 0.5 & \\
\hline$\beta=\frac{\mathrm{L}_{1}}{\mathrm{~L}_{2}}$ & $\frac{1}{4}$ & \\
\hline
\end{tabular}

Tabela 2.1. Valores para estudo numérico do item 2.2.

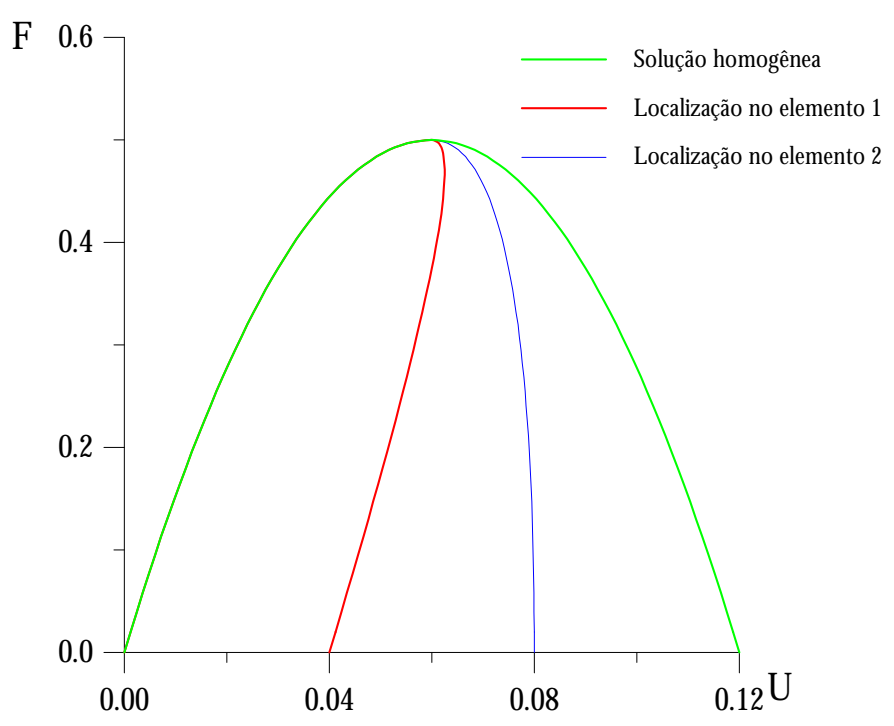

Figura 2.6. Curva FxU para respostas homogênea e localizadas.

A Figura 2.6 ilustra as respostas globais FxU da barra para os três tipos de solução apresentados no item anterior e para os valores da Tabela 2.1. Percebe-se que a localização no elemento 1 provoca snap-badk na resposta global da estrutura, ou seja, 0 elemento que está carregando (1) é menor que o elemento que descarrega (2). Quando ocorre a localização no elemento 20 carregamento prepondera sobre 0 descarregamento elástico e a curva $F_{x} U$ não apresenta o fenômeno do snapbadk. As Figuras 2.7a,b ilustram $\dot{\varepsilon}_{1} / \dot{\varepsilon}_{2}$ x U para 0 caso respostas localizadas nos elementos $1 \mathrm{e}$ 2 , respectivamente. A té a localização, a variação é constante, significando que ambos 
os elementos apresentam carregamento com evolução de dano. Porém, quando um dos elementos inicia 0 descarregamento elástico, a relação $\dot{\varepsilon}_{1} / \dot{\varepsilon}_{2}$ passa por zero e torna-se negativa, uma vez que $\dot{\varepsilon_{1}}$ e $\dot{\varepsilon_{2}}$ tem sinais opostos. Conclui-se, através das Figuras 2.6 e 2.7, que a resposta da estrutura está fortemente ligada à dimensão do elemento.

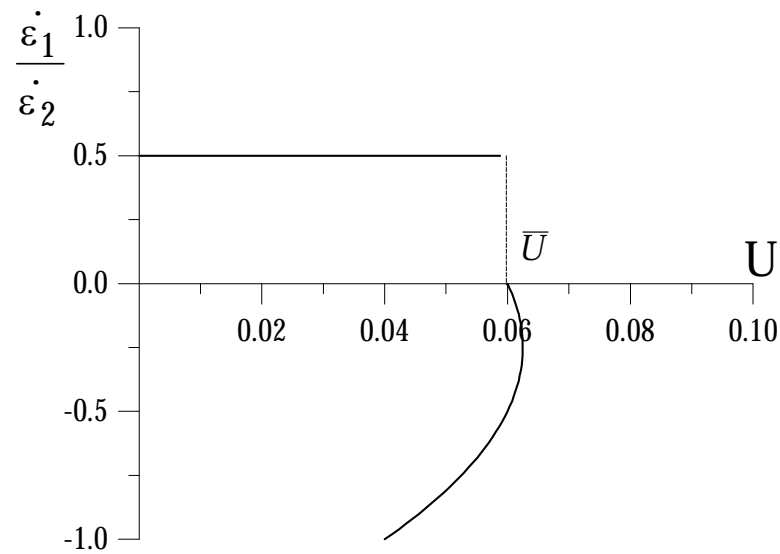

(a)

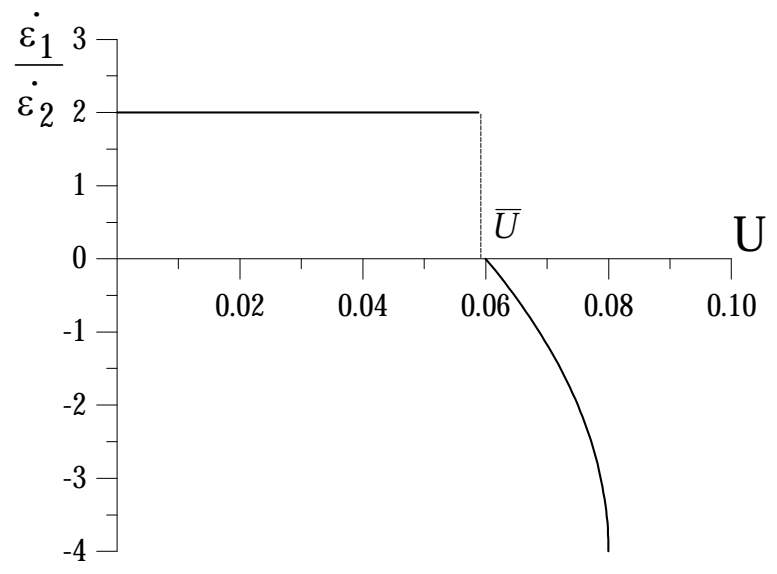

(b)

Figura 2.7. Diagrama da variação relativa de deformação em função do deslocamento total $U$, para (a) localização no elemento 1 e (b) localização no elemento 2.

\subsubsection{Exemplo barra axialmente tracionada}

Neste exemplo considera-se uma barra axialmente tracionada por uma carga aplicada na extremidade livre e engastada na outra extremidade. A barra é discretizada por $\mathrm{n}$ elementos finitos de deformação constante, conforme ilustra a Figura 2.8.

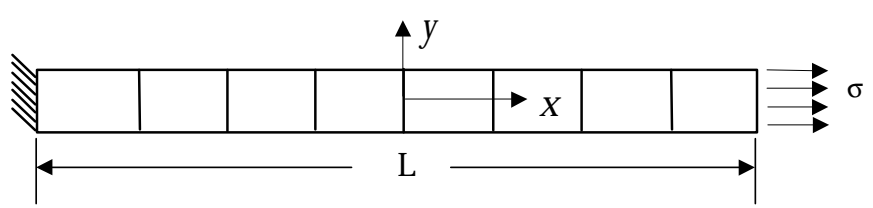

Figura 2.8. Barra sujeita a carregamento uniaxial.

A resposta admitida para 0 material é tal que antes de alcançar a resistência à tração $\bar{\sigma}$ vale uma relação linear entre tensão (normal) $\sigma$ e deformação $\varepsilon$, seguida de um pequeno regime de encruamento positivo próximo da tensão de pico. Depois de alcançada a resistência de pico, vale uma curva de encruamento negativo de acordo com um modelo constitutivo de dano, que será detalhado mais adiante.

Para fins de provocara localização, adota-se para um dos nelementos uma resistência à tração um pouco menor que a dos outros. Uma vez alcançado o limite à tração, num 
processo de deformação controlada, irá ocorrer, nesse elemento, evolução das deformações com redução dos níveis de tensão normal, até o colapso da barra (dano unitário ou próximo dele). Porém, os elementos vizinhos nos quais a resistência à tração não foi excedida, irão descarregar elasticamente. A Figura 2.9 mostra, para 0 caso de $\mathrm{n}=3$, a curva $\sigma \mathrm{X} \varepsilon$ de cada elemento. A Figura $2.9 \mathrm{~b}$ refere-se ao elemento central com resistência à tração menor, que entra em regime de encruamento negativo até a ruptura, enquanto a Figura 2.9a mostra o descarregamento dos demais elementos. Convém ressaltar que os demais elementos descarregam com uma rigidez menor em função da danificação que apresentam já no regime de encruamento positivo. Tomando-se, por outro lado, a deformação média da barra definida pela relação comprimento final sobre inicial, a resposta para vários valores de né mostrada na Figura 2.10.
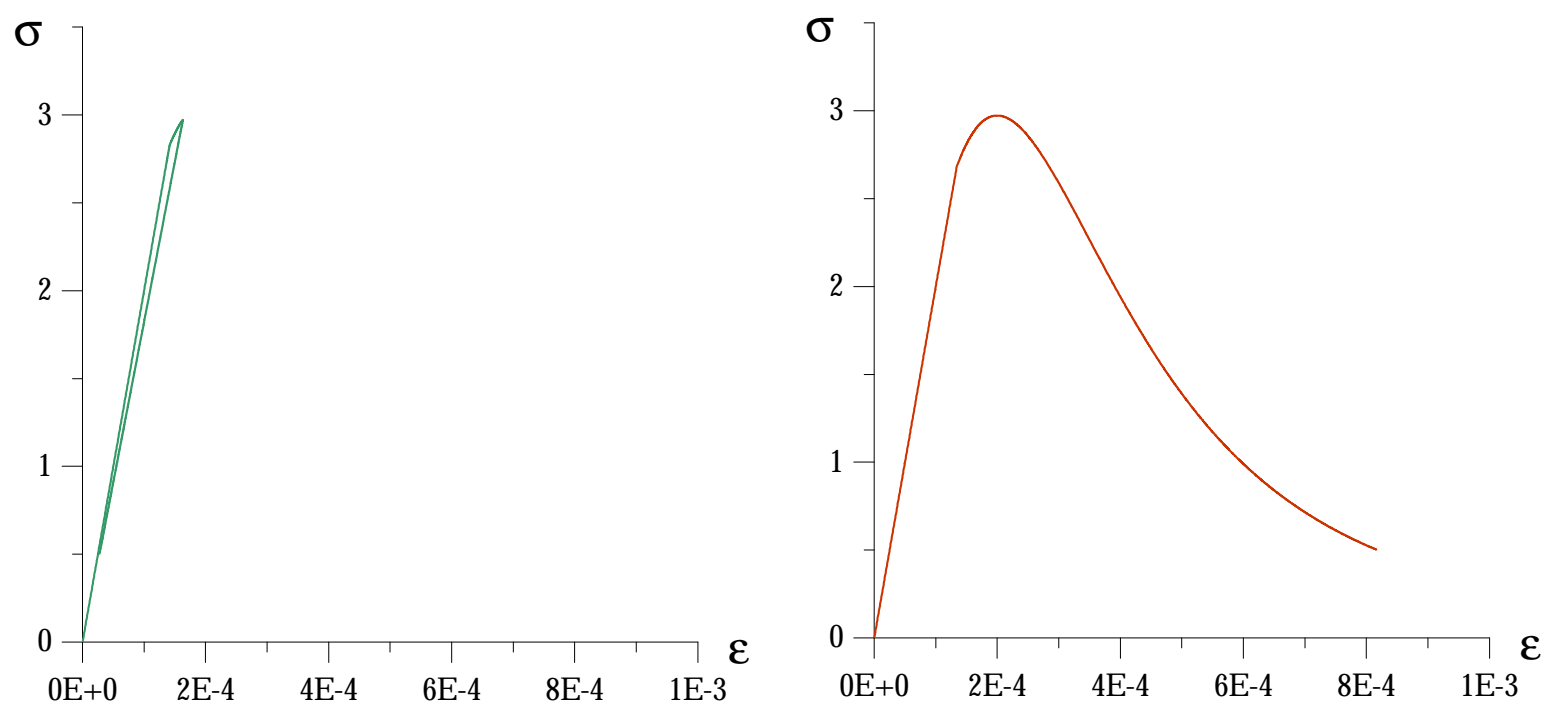

Figura 2.9. Curva $\sigma \mathrm{x} \varepsilon$ durante a análise em elementos finitos para $n=3$ : (a) descarregamento dos elementos 1 e 3; (b) carregamento do elemento 2.

A resposta ilustrada na Figura 2.10 é similar também para a relação cargadeslocamento no ponto de aplicação da carga. Percebe-se que não há objetividade de resultados, isto é, não parece ocorrer convergência para uma curva de ruptura pós pico real. Isto ocorre porque as equações governantes prevêem o mecanismo de ruptura como uma linha, ou zona de localização com espessura zero. A solução por elementos finitos simplesmente tenta captar esta linha localizando as deformações em um único elemento, independente das dimensões deste.

Um resultado que se observa na curva Tensão- Defomaçãomédia é o fenômeno 'snapbadk': o descarregamento elástico proporciona recuperação de deformações que prevalecem sobre aquelas do elemento em carregamento; assim, para um número infinito de elementos $(\mathrm{n} \rightarrow \infty)$ a curva pós - pico coincide com uma curva de descarregamento elástico. 


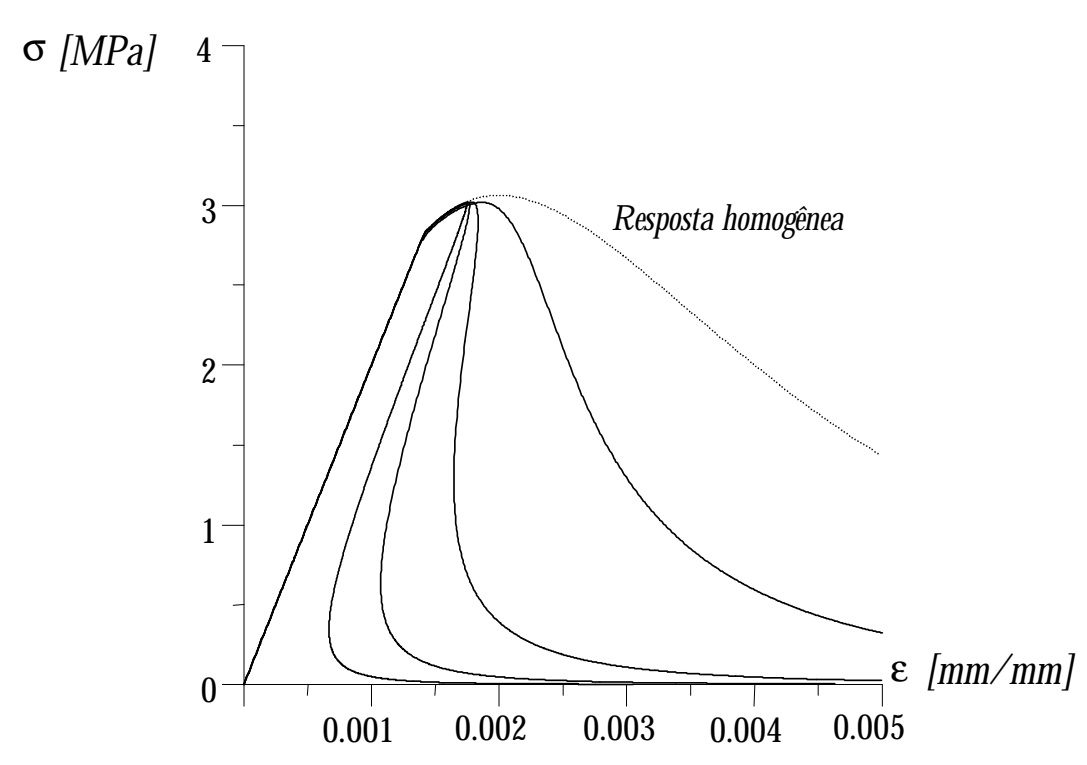

Figura 2.10. Curva Tensão - Deformação média.

Uma outra maneira de interpretar o 'snapboadk' e sua tendência para uma curva de descarregamento elástico quando $\mathrm{n} \rightarrow \infty$ é que neste tipo de simulação a energia dissipada tende a zero com o refinamento da malha, porque a zona na qual 0 processo de ruptura ocorre também tende a zero.

\subsubsection{Exemplo bidimensional}

A Figura 2.11a mostra um espécime analisado por deBarst $\& a{ }^{33}$, Sluys $\& a{ }^{13}{ }^{13}$, Comi \& Peego ${ }^{15}$ com um modelo de plasticidade com gradiente; Com ${ }^{10}$, Com \& Drienie ${ }^{11}$ para 0 caso de modelos de dano com gradiente. Aqui trata-se de ilustrar o problema que surge com uma análise usual através de um modelo de dano local.

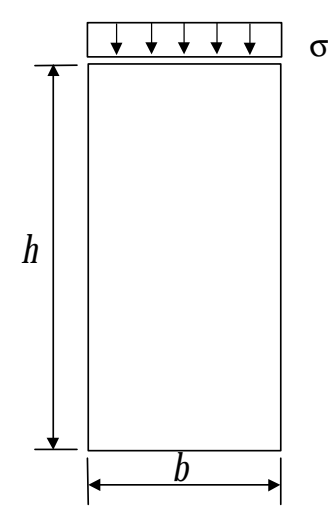

(a)

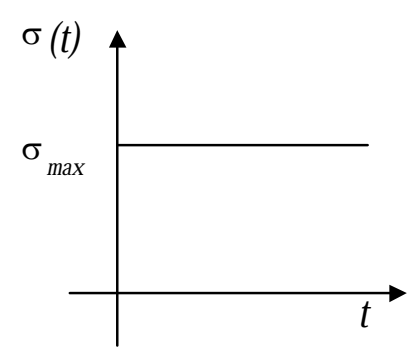

(b)

Figura 2.11. (a) Espécime analisado. (b) Carregamento dinâmico. 
O espécime está sujeito a carregamento de compressão aplicado segundo a lei descrita na Figura 2.11b e foi analisado dinamicamente considerando-se um regime de deformação plana. Para a discretização, usaram-se quatro malhas diferentes de células quadradas formadas por quatro elementos triangulares com deformação constante. Maiores detalhes da análise serão dados no Capítulo 6, quando voltaremos a estudar a mesma estrutura.

A Figura 2.12 mostra os espécimes deformados. Neste caso, a restrição imposta aos deslocamentos em pontos do contorno induz à formação da banda de localização conforme ilustrado. Pode-se perceber que com o refinamento da malha a deformação tende a se concentrar em uma linha de espessura nula em um elemento, levando a resultados diferentes em termos de carga-deslocamento para cada malha adotada.

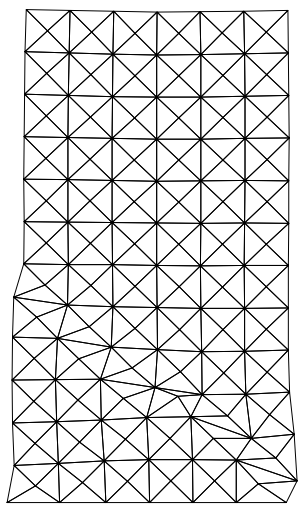

(a)

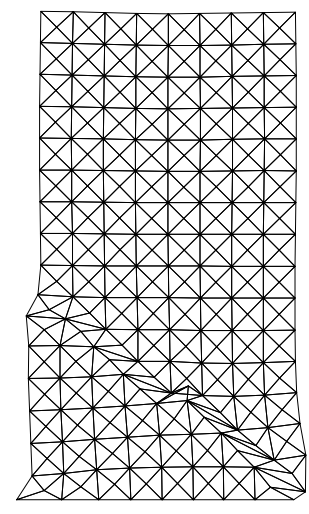

(b)

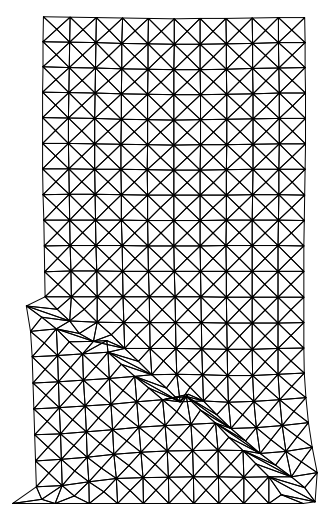

(c)

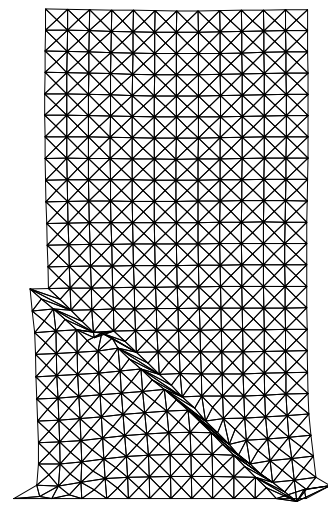

(d)

Figura 2.12. Malhas deformadas ao final da análise.

A Figura 2.13 mostra a distribuição do dano correspondente para cada uma das malhas, ao mesmo instante de tempo em que foram obtidas as deformações ilustradas na Figura 2.12.
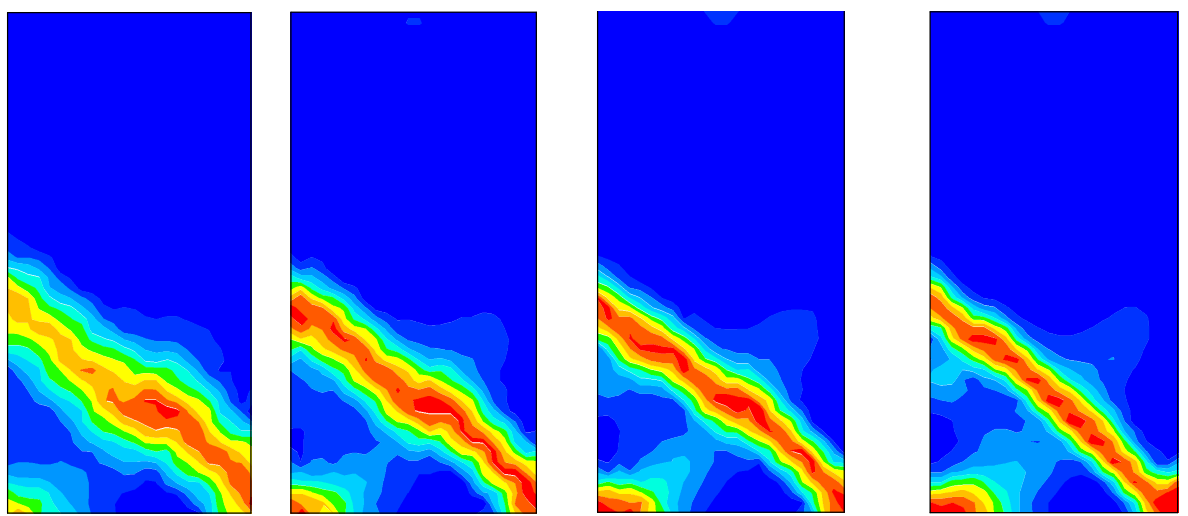

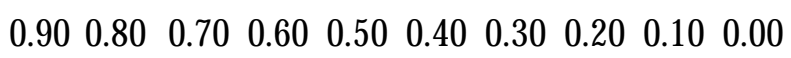

Figura 2.13. Perfil de dano para as malhas (a) 1, (b) 2, (c) 3 e (d) 4. 


\subsection{Métodos de regularização}

Todos os métodos de regularização incluem um comprimento interno, considerado como uma característica do material, que define uma zona onde se concentram as deformações.

\subsubsection{Contínuo Micropolar}

As forças exercidas por uma parte do corpo em outra podem ser representadas, segundo contínuo de Cosserat ou Micropolar: pelo tensor de tensões (tensões normais e de cisalhamento) e, diferentemente do contínuo clássico, inclui-se um par de momentos de tensão, responsáveis pela existência de microcurvaturas suplementares.
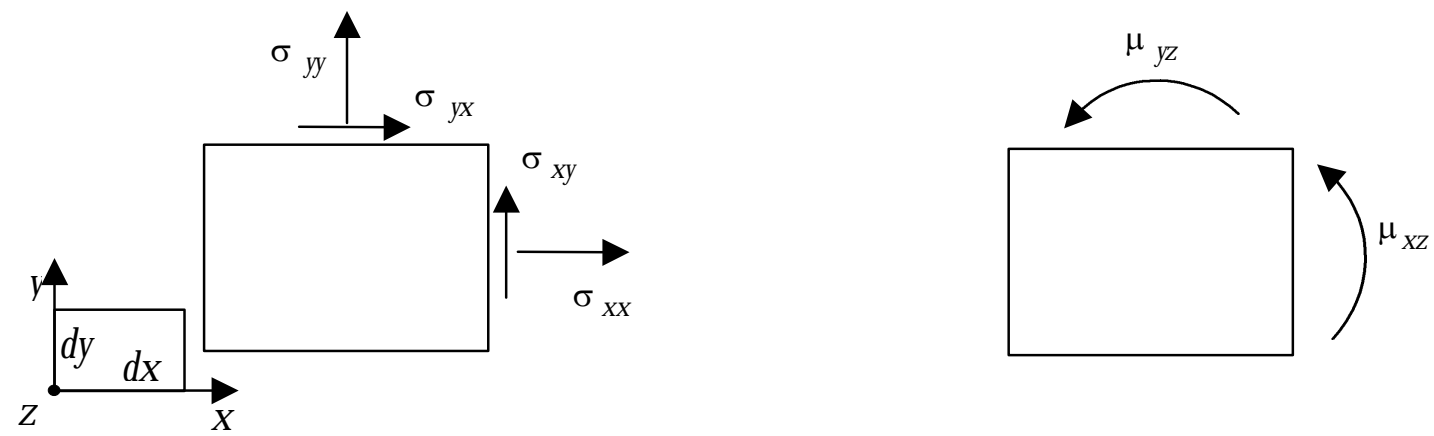

Figura 2.14. Componentes de tensão e pares de momento para o contínuo de Cosserat.

Deste modo, considerando-se os dados da Figura 2.14, as equações de equilíbrio são definidas como:

$$
\begin{aligned}
& \frac{\partial \sigma_{\mathrm{xx}}}{\partial \mathrm{x}}+\frac{\partial \sigma_{\mathrm{yx}}}{\partial \mathrm{y}}=0 \\
& \frac{\partial \sigma_{\mathrm{xy}}}{\partial \mathrm{x}}+\frac{\partial \sigma_{\mathrm{y}}}{\partial \mathrm{y}}=0 \\
& \frac{\partial \mu_{\mathrm{xz}}}{\partial \mathrm{x}}+\frac{\partial \mu_{\mathrm{yz}}}{\partial \mathrm{y}}-\left(\sigma_{\mathrm{yx}}-\sigma_{\mathrm{xy}}\right)=0
\end{aligned}
$$

Com a última equação, percebe-se que o tensor de tensões é simétrico se: $\frac{\partial \mu_{\mathrm{xz}}}{\partial \mathbf{x}}+\frac{\partial \mu_{\mathrm{yz}}}{\partial \mathbf{y}}=0$. 
Uma vez que foram adicionadas componentes relacionadas ao par de momentos no tensor de tensões, deve-se incluir graus de liberdade rotacionais $\mathrm{w}$ independentes dos deslocamentos u, para um compatível tensor de deformações. D essa forma, o tensor de deformações $\varepsilon$ é construído com a contribuição das micro - curvaturas $\mathrm{k}$, que derivam do gradiente das micro - rotações, além da clássica contribuição do gradiente de deslocamentos. Para o caso bidimensional:

$$
\mathrm{k}_{\mathrm{zx}}=\frac{\partial \mathrm{w}_{\mathrm{z}}}{\partial \mathrm{x}}, \quad \mathrm{k}_{\mathrm{zy}}=\frac{\partial \mathrm{w}_{\mathrm{z}}}{\partial \mathrm{y}}
$$

Com respeito às tensões, admitindo-se isotropia e que $\mu_{\mathrm{zx}}$ e $\mu_{\mathrm{zy}}$ são proporcionais às respectivas micro - curvaturas:

$$
\mu_{\mathrm{zx}}=\mathrm{G} \ell^{2} \mathrm{k}_{\mathrm{zX}}, \quad \mu_{\mathrm{zy}}=\mathrm{G} \ell^{2} \mathrm{k}_{\mathrm{zy}},
$$

onde $\mathrm{G}$ é 0 módulo elástico de cisalhamento e $\ell$ é um comprimento interno do material.

Deste modo, as componentes de deformação $\mathrm{k}_{\mathrm{zx}} \ell$ e $\mathrm{k}_{\mathrm{zy}} \ell$ e tensão $\mu_{\mathrm{zx}} / \ell$ e $\mu_{\mathrm{zx}} / \ell$ devem ser acrescidas aos termos de distorção angular e tensão de cisalhamento dos tensores clássicos de deformações e tensões, respectivamente.

A teoria elástica do contínuo de Cosserat pode ser estendida para elasto-plasticidade e aplicada na análise de localização de deformações. A presença de micro - rotações é especialmente relevante para 0 caso de materiais granulares como 0 solo, e 0 comprimento interno determina a espessura das bandas de cisalhamento, conforme Mïhlhaus \& V ardaulakis ${ }^{34}$.

A aplicação da teoria é limitada, entretanto, aos casos em que os graus de liberdade rotacionais estão ativos durante a deformação. Portanto, a regularização ocorre para problemas com cisalhamento dominante e não serve para o caso de tensão pura.

\subsubsection{Integral não - local}

Segundo Bazant \& al. ${ }^{5}$, o modelo não local trata as variáveis de estado não mais como funções exclusivas das variáveis no ponto, mas como uma integral média destas variáveis em uma certa vizinhança:

$$
\mathrm{Y}(\mathrm{x})=\int_{\mathrm{V}} g(\mathrm{~s}) \mathrm{Y}(\mathrm{x}+\mathrm{s}) \mathrm{dV}
$$


onde $\bar{Y}$ é a variável de estado não - local e g(s) uma função que preserve a noção de caráter não - local de uma lei constitutiva. Uma escolha possível para g(s) é a clássica função peso (deBarst \& Mühlhaus ${ }^{29}$ ):

$$
g(s)=\frac{\ell}{2 \sqrt{\pi}} \exp \left(\frac{-\ell^{2} s^{2}}{4}\right)
$$

de modo que à medida que aumenta a distância sdo ponto considerado, o efeito nãolocal diminui. 0 parâmetro $\ell$ representa uma escala interna característica do material, com a dimensão de comprimento. Este parâmetro determina a dimensão da vizinhança do ponto $\mathrm{x}$ que afeta a função não local $\mathrm{Y}$.

Deste modo, a equação 2.37 pode ser rescrita de modo que:

$\mathrm{Y}(\mathrm{x})=\frac{\ell}{2 \sqrt{\pi}} \int_{-\infty}^{+\infty} \mathrm{e}^{-\frac{\ell^{2} \mathrm{~s}^{2}}{4}} \mathrm{Y}(\mathrm{x}+\mathrm{s}) \mathrm{ds}$

Quando $\mathrm{Y}(\mathrm{x}+\mathrm{s})$ é desenvolvido em uma série de Taylor por volta de $\mathrm{s}=0$, a equação anterior passa a ser escrita da forma:

$\mathrm{Y}(\mathrm{x})=\frac{\ell}{2 \sqrt{\pi}}\left[\int_{-\infty}^{+\infty} \mathrm{e}^{-\frac{\ell^{2} \mathrm{~s}^{2}}{4}} \mathrm{Y}(\mathrm{x}) \mathrm{ds}+\int_{-\infty}^{+\infty} \mathrm{se} \mathrm{e}^{-\frac{\ell^{2} \mathrm{~s}^{2}}{4}} \frac{\mathrm{dY}(\mathrm{x})}{\mathrm{dx}} \mathrm{ds}+\int_{-\infty}^{+\infty} \frac{\mathrm{s}^{2}}{2} \mathrm{e}^{-\frac{\ell^{2} \mathrm{~s}^{2}}{4}} \frac{\mathrm{d}^{2} \mathrm{Y}(\mathrm{x})}{d \mathrm{x}^{2}} \mathrm{ds}+\ldots\right]$

D esprezando-se termos de ordem superior, pode-se escrever $\bar{Y}$ como:

$\bar{Y}(\mathrm{x})=\mathrm{Y}(\mathrm{x})+\frac{1}{\ell^{2}} \frac{\mathrm{d}^{2} \mathrm{Y}(\mathrm{x})}{\mathrm{dx}^{2}}$

Para $\mathrm{Y}$ representando, por exemplo, a deformação plástica $\varepsilon^{\mathrm{p}}$, tem-se que:

$\bar{\varepsilon}^{\mathrm{p}}(\mathrm{x})=\varepsilon{ }^{\mathrm{p}}(\mathrm{x})+\frac{1}{\ell^{2}} \frac{\mathrm{d}^{2} \varepsilon \mathrm{p}^{\mathrm{p}}(\mathrm{x})}{\mathrm{dx}^{2}}$ 
A equação anterior é dita nãolocal, pois inclui gradientes da variável plástica, de modo que 0 valor da deformação plástica em um ponto depende de uma vizinhança deste ponto. Estes termos de ordem superior agem como estabilizadores e garantem a elipticidade das equações estáticas governantes. Motivações físicas para 0 uso de modelo não local encontra-se em Bazant ${ }^{35}$.

\subsubsection{Energia de fratura}

Esta técnica se baseia no critério de Ginffith sobre a propagação de uma fratura discreta que consiste em admitir que a formação de uma macrofissura requer uma certa quantidade de energia, por unidade área da zona fraturada, considerada uma propriedade do material e chamada de energa defratura.

Tendo-se em vista os resultados apresentados no item 2.3, e que mostram uma tendência de reprodução de uma dissipação nula em correspondência à ruptura segundo uma linha, compreende-se que a imposição da energia de fratura como uma propriedade do material funciona como um limite inferior à dissipação simulada. Isto equivale a impor uma zona de localização cuja espessura é definida por um comprimento interno característico, conforme se mostra no que segue.
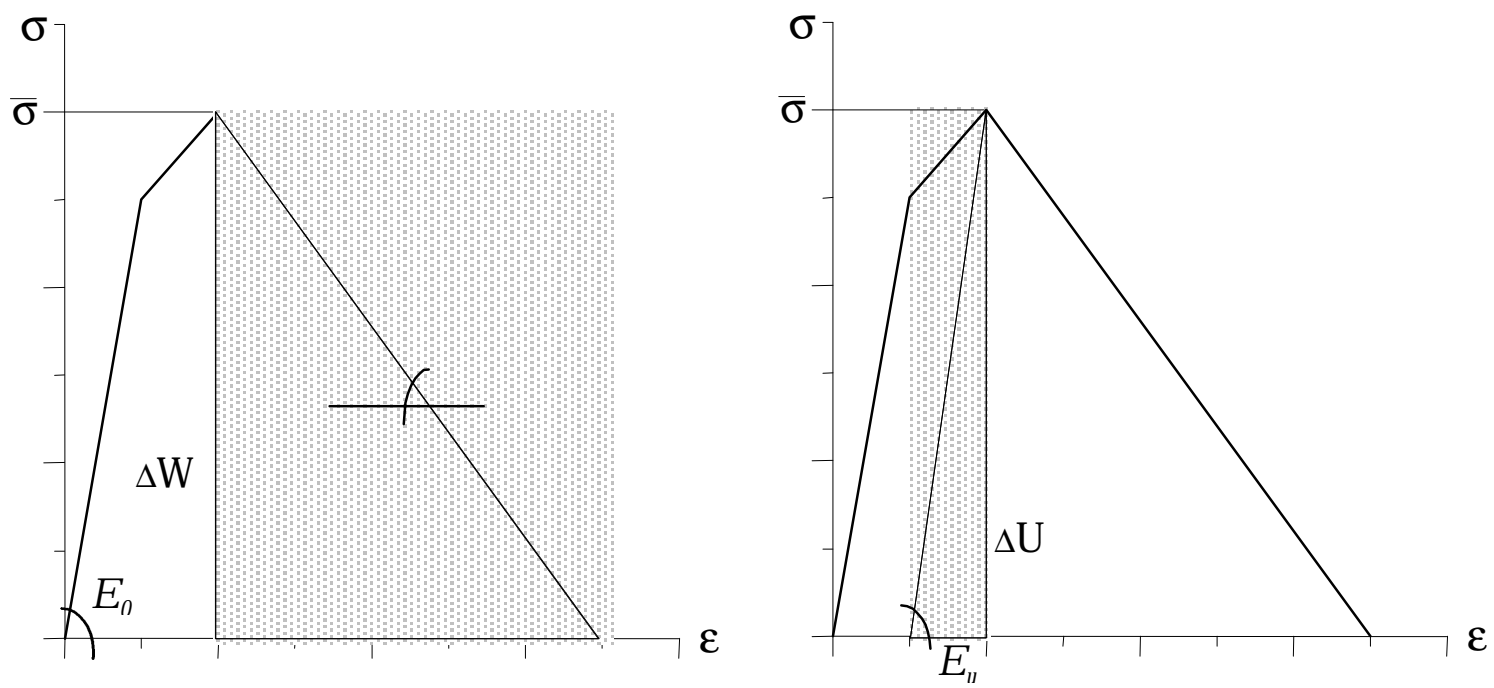

Figura 2.15. $G_{f}=(\Delta W)+(\Delta U)$, para $o$ caso de softening linear.

Matematicamente, esta energia é igual a área sob as curvas de carregamento e descarregamento saindo do pico de tensão, conforme Figura 2.15 (Oliver ${ }^{32}$, Bazant \& Codbin ${ }^{36}$ ). Em Bazant \& Cedbin ${ }^{36}$ divide-se esta área em $\Delta \mathrm{W}$ (trabalho realizado em regime sattening e $\Delta \mathrm{U}$ (energia dissipada pela microfissuração durante 0 hardkning. De acordo com a definição:

$\frac{\mathrm{G}_{\mathrm{f}}}{\mathrm{S}}=\Delta \mathrm{W}+\Delta \mathrm{U}$ 
onde s é uma dimensão igual à espessura da zona de localização, denominado comprimento característico.

No caso de satteninglinear e $E_{u} \cong E_{0}=E$, a Figura 2.15 e a expressão 2.43 fornecem:

$$
\begin{aligned}
& \Delta \mathrm{W}=\bar{\sigma} \frac{\bar{\sigma}}{2 \mathrm{~h}} \quad \Delta \mathrm{U}=\bar{\sigma} \frac{\bar{\sigma}}{2 \mathrm{E}} \\
& \therefore \mathrm{G}_{\mathrm{f}}=\frac{\bar{\sigma}^{2} \mathrm{~S}}{2}\left(\frac{1}{\mathrm{~h}}+\frac{1}{\mathrm{E}}\right)
\end{aligned}
$$

Da relação anterior segue que o módulo de encruamento negativo hé dado por:

$$
\frac{1}{h}=\frac{2 G_{f}}{s^{-2}}-\frac{1}{E}
$$

Nas simulações numéricas, a técnica de regularização em questão consiste em associar sa uma das dimensões do elemento finito adotado (quando se trata de elemento com deformação constante). Nessas condições 0 módulo h passa a ser definido pela 2.45 para cada malha, garantindo-se $\mathrm{G}_{\mathrm{f}}$ constante e contornando-se o problema da dependência de discretização. Analisando novamente a barra da Figura $2.8 \mathrm{com} \mathrm{n}$ elementos, sendo um elemento com resistência a tração menor que os demais, a deformação média ao longo da barra vale:

$$
\varepsilon=\frac{\sigma}{\mathrm{E}}+\frac{(\sigma-\bar{\sigma})}{\mathrm{n}}\left(\frac{1}{\mathrm{~h}}+\frac{1}{\mathrm{E}}\right)
$$

e obtém-se a seguinte relação tangencial entre a tensão $\sigma$ e deslocamento una barra:

$$
\frac{\partial(\mathrm{u} / \mathrm{L})}{\partial \sigma}=\frac{1}{\mathrm{E}}+\frac{1}{\mathrm{n}}\left(\frac{1}{\mathrm{~h}}+\frac{1}{\mathrm{E}}\right)
$$

A localização resultante da análise clássica em elementos finitos ocorre em um único elemento, ou seja, $\mathrm{s}=\mathrm{L} / \mathrm{n}$ Usando a equação 2.45 , pode-se rescrever a equação anterior como:

$$
\frac{\partial(\mathrm{u} / \mathrm{L})}{\partial \sigma}=\frac{1}{\mathrm{E}}+\frac{2 \mathrm{G}_{\mathrm{f}}}{\mathrm{L} \bar{\sigma}^{2}}
$$


que mostra que a solução pós - pico é dependente dos parâmetros do material $\mathrm{E}, \mathrm{G}_{\mathrm{f}}$ $\bar{\sigma}$ e do comprimento estrutural L. Quando se utiliza o parâmetro $\mathrm{G}_{\mathrm{f}}$ como um parâmetro adicional, a resposta global Fơça x Dedocamento se torna independente da discretização.

Entretanto, localmente nada foi alterado e a localização ainda ocorre apenas em uma faixa de elementos, apesar da energia dissipada permanecer constante. Por este motivo, Pamin ${ }^{14}$ e deBarst $\&$ al. ${ }^{37}$ referem-se à energia de fratura como um truquede regularização.

\subsubsection{Modelos com gradiente}

$\mathrm{Na}$ teoria clássica, a função de escoamento $\mathrm{f}$ tem sua evolução governada por uma medida da deformação plástica equivalente $\gamma$. Para situações de encruamento negativo a forma local do modelo constitutivo é apropriada desde que as deformações plásticas se distribuam de modo difuso no meio, e formas específicas de $\mathrm{f}(\gamma)$ são justificadas pela própria observação da microestrutura. Enquanto para muitos casos esta consideração apresenta boa aproximação, isto não ocorre quando a deformação, a partir de um certo valor, passa a se localizar em uma zona estreita e a homogeneidade é perdida com relação à escala do elemento. Para levar em conta 0 efeito da distribuição heterogênea da deformação, a teoria do gradiente propõe que $\mathrm{f}$ passe a depender também de gradientes de $\gamma$. Portanto, os termos com gradiente complementam a teoria clássica local com informações sobre 0 comportamento do material em uma escala menor em torno do ponto considerado.

Muiblhaus \& Aifantis ${ }^{14}$ demonstram que os modelos não locais são um caso particular dos modelos com gradiente.

Vários autores consideram ainda que os efeitos do tempo no modelo constitutivo regularizam 0 problema. Com \& Peeeg ${ }^{38}$ apresentam um modelo de dano simplificado com viscosidade, e mostram que a resposta ainda apresenta dependência dos resultados na discretização da estrutura para valores de dano maiores que aproximadamente 0.5. Dubé $\notin$ al. ${ }^{39,40}$ demostram que a introdução da viscosidade impossibilita a formação de ondas estacionárias (assunto do próximo capítulo). Needeman ${ }^{41}$ demonstrou a existência de um comprimento característico que é introduzido implicitamente pelo modelo viscoso. 


\section{MÉTODO DE REGULARIZACÃO COM GRADIENTES}

\subsection{Introdução}

Considere-se o seguinte conjunto de equações:

$\mathrm{C}^{\mathrm{T}} \sigma=\rho \mathrm{u}$

$\varepsilon=\mathrm{Cu}$

$\sigma=\mathrm{E} \varepsilon^{\mathrm{e}} \quad \varepsilon=\varepsilon^{\mathrm{e}}+\varepsilon^{\mathrm{p}}$ $a, b, c, d, e, f)$

$\dot{\varepsilon}^{\mathrm{p}}=\dot{\lambda} \mathrm{n}$

$\mathrm{f}(\sigma, \lambda) \leq 0 \quad \dot{\lambda} \geq 0 \quad \hat{\mathrm{\lambda}}=0$

$\mathrm{f}=0$

que define 0 problema elasto - plástico em sua forma local. $\mathrm{Na}$ equação de equilíbrio dinâmico (3.1a) $\sigma$ é um vetor que contém as componentes de tensão, C é a matriz dos operadores diferenciais e $\rho$ é a densidade. Para pequenos gradientes de deslocamento a matriz $\mathrm{C}$ pode ser também usada para relacionar as componentes de deformação, reunidas no vetor $\varepsilon$, com 0 vetor deslocamentos $u$ conforme indica a equação de compatibilidade 3.1b. A equação 3.1c reúne tensão e deformação dástica relacionados pelo tensor constitutivo de rigidez elástica E. No campo das pequenas deformações, estas podem ser divididas em uma parcela elástica e outra plástica. Em 3.1d, empregando-se uma lei associativa, né um versor normal à superfície de plastificação $\mathrm{f}=0$ determinado por $\mathrm{n}=\partial \mathrm{f} / \partial \sigma$, e $\dot{\lambda}$ é um 
escalar não negativo representativo da intensidade da taxa de deformação plástica. As condịôes de complementanidade 3.1e e de consistênia de Prager 3.1f, permitem determinar $\dot{\lambda}$ de modo que as condições de carregamento plástico ou descarregamento elástico estejam satisfeitas.

\subsection{Propagação de ondas}

0 fenômeno da localização de deformações em meios homogêneos tem suas características evidenciadas pelo estudo das condições de propagação e estacionariedade de ondasno meio.

D e um modo geral, ondas referem-se a perturbações que se propagam ao interno do material. Em termos matemáticos o vetor deslocamento de um ponto provocado por uma onda é definido por uma expressão do tipo

$\mathrm{u}=\mathrm{u}(\overline{\mathrm{n}} \cdot \mathrm{x}-\mathrm{d}) \mathrm{m}$

onde ué uma função em princípio de classe $\mathrm{C}^{\infty}$, dependente do vetor $\mathrm{x}$, reunindo as coordenadas do ponto, e do tempo t- Claugh ${ }^{42}$, Whitham ${ }^{43}$, Bland ${ }^{44}$. As direções n e $\mathrm{m}$ representam a direção de propagação e a direção do deslocamento, respectivamente; cé a velocidade de propagação do fronte de onda F. A função $\phi(\tilde{n} \cdot \mathrm{x}-\mathrm{d})$ é genericamente denominada função de onda simples (a forma da perturbação não se altera durante a propagação no meio), e 0 argumento $\widehat{n} \cdot \mathbf{x}$ - đt é a fase da função de onda. A Figura 3.1 ilustra as considerações em questão.

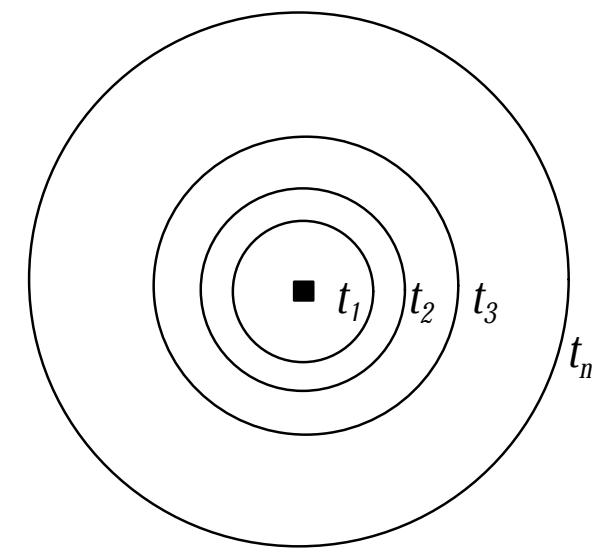

(a)

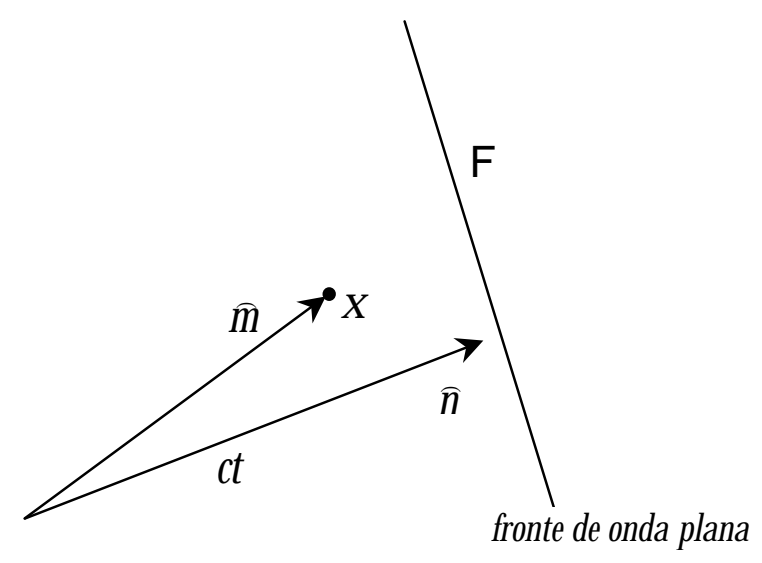

(b)

Figura 3.1. (a) Propagação do fronte de uma onda. (b) $E m t_{n} \rightarrow \infty$, a onda pode ser considerada como plana.

Para ilustrar analiticamente 0 problema da localização associado a propagação de onda em meio elastoplástico, reproduz-se o problema analisado por Bazant \& 
Beytschko $0^{45}$. Trata-se de uma barra de comprimento 2L, com seção transversal unitária e densidade $\rho$, submetida a deslocamentos impostos nas extremidades livres. A Figura 3.2 ilustra a geometria da barra, a forma de carregamento e 0 diagrama tensão- defomaçãa

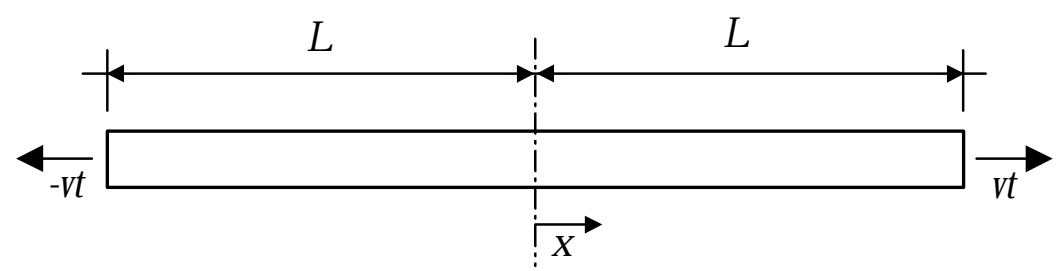

(a)

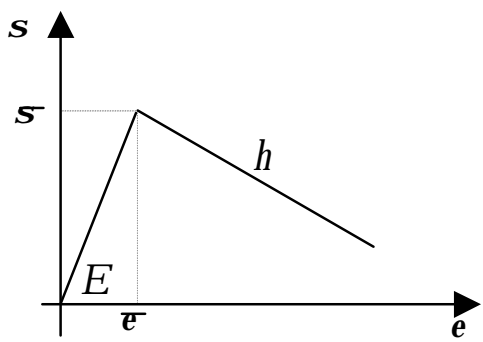

(b)

Figura 3.2.a) Barra analisada, com deslocamento imposto vt nas extremidades livres, b)Lei constitutiva com encruamento negativo.

Supondo que a barra não ultrapasse seu limite elástico, as equações 3.1a,b,c podem ser combinadas na forma da equação de onda elástica:

$\frac{\rho}{E} \frac{\partial^{2} u}{\partial t^{2}}-\frac{\partial^{2} u}{\partial x^{2}}=0$

onde, para o caso unidimensional $\mathrm{u}=\mathrm{u}(\mathrm{x}, \mathrm{t})$.

Admitindo-se que a barra esteja inicialmente em repouso e impondo-se as condições de contorno:

$\mathrm{u}(\mathrm{L}, \mathrm{t})=\mathrm{t}$

$\mathrm{u}(-\mathrm{L}, \mathrm{t})=-\mathrm{t}$

para $t \geq 0$, a solução da equação 3.3 é:

$u=-v\left(t-\frac{L+x}{C_{e}}\right)+v\left(t-\frac{L-x}{C_{e}}\right)$

onde $\langle\bullet\rangle=\bullet$ se $\bullet>0$ e $\langle\bullet\rangle=0$ se $\bullet \leq 0$. Para 0 caso elástico $\left(\mathrm{v} / \mathrm{c}_{\mathrm{e}}<\bar{\varepsilon} / 2\right)$, a equação 3.5 é válida para $\mathrm{t} \leq 2 \mathrm{~L} / \mathrm{c}_{\mathrm{e}}$, ou seja, até o instante em que as duas ondas percorreram todo o comprimento da barra. 
Considerando-se que a origem do sistema de referência está no centro da barra, a solução se apresenta na forma de duas ondas arbitrárias, cada uma se propagando da extremidade para o centro da barra com velocidade $C_{\mathrm{e}}=\sqrt{\mathrm{E} / \rho}$.

Para $\varepsilon=\partial \mathrm{u} / \partial \mathrm{x}$ (conforme 3.1b):

$\varepsilon=\frac{\mathrm{v}}{\mathrm{C}_{\mathrm{e}}}\left[\mathrm{H}\left(\mathrm{t}-\frac{\mathrm{L}+\mathrm{x}}{\mathrm{C}_{\mathrm{e}}}\right)+\mathrm{H}\left(\mathrm{t}-\frac{\mathrm{L}-\mathrm{x}}{\mathrm{C}_{\mathrm{e}}}\right)\right]$

onde $\mathrm{H}$ representa a função de Heaviside $(\mathrm{H}(\bullet)=1$ se $\bullet>0 ; \mathrm{H}(\bullet)=0$ se $\bullet \leq 0)$.

Para o desenvolvimento que segue, é importante definir:

$\mathrm{t}_{1}=\frac{\mathrm{L}-\mathrm{x}}{\mathrm{C}_{\mathrm{e}}} \quad \mathrm{t}_{2}=\frac{\mathrm{L}+\mathrm{x}}{\mathrm{C}_{\mathrm{e}}}$

os tempos que as ondas levam para sair da extremidade direita e esquerda da barra, respectivamente, e chegar ao ponto de coordenada $\mathrm{x}$.

Para um instante de tempo tonde $t>t_{1}$ e $t<t_{2}$, por exemplo:

$$
\begin{aligned}
& \mathrm{u}=\mathrm{v}\left(\mathrm{t}-\mathrm{t}_{1}\right) \\
& \varepsilon=\frac{\mathrm{v}}{\mathrm{C}_{\mathrm{e}}}
\end{aligned}
$$

ue $\varepsilon$ recebem a influência somente da onda que saiu da extremidade direita, pois a onda que se propaga a partir da extremidade esquerda ainda não atingiu o ponto de coordenada $\mathrm{x}$, no instante t. Deste modo, enquanto as ondas não se encontram, $\mathrm{u}$ cresce linearmente com 0 tempo e $\varepsilon$ permanece constante no ponto.

Para $t>t_{1}$ e $t>t_{2}$

$$
u=v\left(t-t_{1}\right)-v\left(t-t_{2}\right)
$$

$\varepsilon=2 \frac{\mathrm{V}}{\mathrm{C}_{\mathrm{e}}}$

o deslocamento é uma combinação linear da influência das duas ondas e a deformação dobra seu valor. 
Para estudar a localização, supõe-se agora que o limite elástico seja ultrapassado quando as ondas se encontrarem em $\hat{x}=0$, ou em $\hat{t}=L / c_{e}$.

No caso de resposta elastoplástica e impondo-se um modelo linear de encruamento negativo, o critério de plastificação pode ser escrito na forma:

$$
\mathrm{f}=\sigma-\bar{\sigma}-\mathrm{h}^{\mathrm{p}}
$$

com $\mathrm{h}<0$.

Conforme 3.1b,c e impondo-se ainda $\mathrm{f}=0$, a variação $\partial \sigma / \partial \mathrm{x}$ pode ser escrita como:

$\frac{\partial \sigma}{\partial \mathrm{x}}=\frac{\partial \sigma}{\partial \varepsilon} \frac{\partial \varepsilon}{\partial \mathrm{x}}=\frac{\mathrm{Eh}}{\mathrm{E}+\mathrm{h}} \frac{\partial^{2} \mathrm{u}}{\partial \mathrm{x}^{2}}$

Substituindo-se 3.11 em 3.1a tem-se , portanto, a equação de propagação de onda para uma barra plastificada:

$$
\frac{E+h}{C_{e}^{2}} \frac{\partial^{2} u}{\partial t^{2}}-h \frac{\partial^{2} u}{\partial x^{2}}=0
$$

Sendo $\mathrm{h}>0$ tem-se uma resposta real e a equação 3.12 se mantém hiperbólica, para $\mathrm{h}=0$ a velocidade de fase é nula e para encruamento negativo $(\mathrm{h}<0)$, a velocidade de fase é imaginária e a equação 3.12 passa a ser elíptica. Ondas com velocidade imaginária não se propagam, portanto, na medida em que a resposta do meio passa para um regime de encruamento negativo, o seu efeito passa a ser localizado não se estendendo e permanecendo numa vizinhança infinitamente pequena.

A Figura 3.3 mostra a interface entre as regiões elástica $\Omega{ }^{\mathrm{e}}$ e plástica $\Omega^{\mathrm{s}}$, situada a uma distância fixa vt da extremidade esquerda da barra, onde $\widetilde{\nabla}$ é a velocidade da interface. L é a distância entre a extremidade esquerda da barra e P é um ponto à direita da interface. 


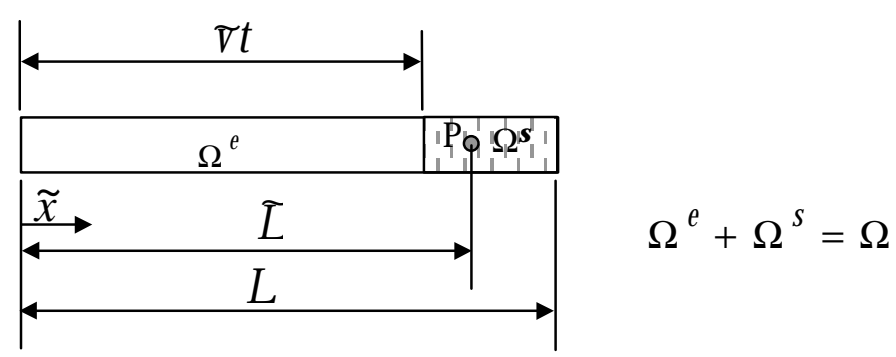

Figura 3.3. Região plástica $\left(\Omega^{\mathrm{s}}\right)$, elástica $\left(\Omega^{\mathrm{e}}\right)$ e a interface entre as regiões.

Os deslocamentos de pontos à direita e à esquerda da interface são dados, respectivamente, por:

$u^{s}=\tilde{u}+\varepsilon^{s}(\tilde{x}-\tilde{v} t)$

$u^{e}=\tilde{u}-\varepsilon^{e}(\tilde{v} t-\tilde{x})$

onde $\tilde{u}$ é o deslocamento na interface, $\varepsilon^{\mathrm{s}}$ e $\varepsilon^{\mathrm{e}}$ são as deformações imediatamente à direita e à esquerda da interface, respectivamente. E suas derivadas materiais:

$$
\begin{aligned}
& \dot{\mathrm{u}}^{\mathrm{S}}=\dot{\tilde{\mathrm{u}}}-\widetilde{\mathrm{v} \varepsilon} \mathrm{s} \\
& \dot{\mathrm{u}}^{\mathrm{e}}=\dot{\tilde{\mathrm{u}}}-\widetilde{\nabla} \varepsilon
\end{aligned}
$$

são as velocidades materiais no ponto. 0 balanço da quantidade de movimento linear para meios contínuos postula que a taxa de vaniação da quantidade total de moimento no demento éigual ao vetor soma detodas as forças atuantes netedementa $\mathrm{O} u$ seja, para o elemento de comprimento $\tilde{\mathrm{L}}$, tem-se:

$\frac{\partial}{\partial \mathrm{t}}\left[\int_{0}^{\nabla t} \rho\left(\dot{\tilde{u}}-\widetilde{\nabla} \varepsilon^{e}\right) d \widetilde{x}+\int_{\nabla t}^{\widetilde{L}} \rho\left(\dot{\tilde{u}}-\widetilde{\nabla} \varepsilon^{s}\right) d \widetilde{x}\right]=\sigma^{s}-\sigma^{e}$

Assim, obtém-se uma relação para o salto de tensões e deformações na interface:

$$
\sigma^{s}-\sigma^{e}=\rho \widetilde{\nabla}^{2}\left(\varepsilon^{s}-\varepsilon^{e}\right)
$$

Seja ainda 2sa largura da zona central de localização, adjacente a $\mathbf{x}=0$, com valor inicial $\widehat{s}=0$ em $t=\hat{t}$. Para s pequeno, pode-se admitir a deformação $\varepsilon$ s na zona de localização como sendo constante, e, portanto: 


$$
\begin{array}{ll}
\mathrm{u}^{\mathrm{S}}=\varepsilon^{\mathrm{s}} \mathrm{x} & -\mathrm{s} \leq \mathrm{x} \leq \mathrm{s}, \mathrm{t}>\hat{\mathrm{t}} \\
\mathrm{u}^{\mathrm{e}}=\mathrm{y}\left(\frac{\mathrm{L}+\mathrm{x}}{\mathrm{C}_{\mathrm{e}}}-\mathrm{t}\right)+\varphi(\mu), \mu=\mathrm{t}-\frac{\mathrm{L}-\mathrm{x}}{\mathrm{C}_{\mathrm{e}}} & -\mathrm{C}_{\mathrm{e}}(\mathrm{t}-\hat{\mathrm{t}}) \leq \mathrm{x}<-\mathrm{s}
\end{array}
$$

onde $\varphi$ é uma função arbitrária que descreve a propagação da onda na direção da extremidade esquerda da barra. A equação 3.17a define o deslocamento na zona plástica enquanto $3.17 \mathrm{~b}$ define o deslocamento na zona elástica. Deste modo, a deformação na parte elástica é dada por:

$\varepsilon^{\mathrm{e}}=\frac{1}{\mathrm{C}_{\mathrm{e}}}\left(\mathrm{V}+\varphi^{\prime}(\mu)\right) \quad-\mathrm{C}_{\mathrm{e}}(\mathrm{t}-\hat{\mathrm{t}}) \leq \mathrm{x}<-\mathrm{S}$

$\operatorname{com} \varphi^{\prime}(\mu)=d \varphi(\mu) / d \mu$.

Usando as condiçoes deinteface, impõe-se continuidade de deslocamentos em x = -s:

$$
\varepsilon s=\frac{1}{s}\left[y\left(\frac{L-s}{C_{e}}-t\right)+\varphi\left(\mu^{s}\right)\right], \quad \quad \mu^{s}=t-\frac{L+s}{C_{e}}
$$

Sabe-se que as tensões na interface devem obedecer à condição 3.16. 0 plano da interface no lado esquerdo da barra (conforme Figura 3.3), pode movimentar-se para esquerda, ou permanecer estacionária $(\mathrm{s} \rightarrow 0)$. Supondo a primeira opção, tem-se pontos entrando em regime satteninga medida em que a interface se move. Para que isso ocorra, $\sigma^{\mathrm{e}}=\bar{\sigma}$ e, portanto, $\sigma^{\mathrm{e}} \geq \sigma^{\mathrm{s}}$. Porém, $\varepsilon^{\mathrm{e}}<\bar{\varepsilon}<\varepsilon$, pois a deformação deve ser maior que $\bar{\varepsilon}$ dentro da zona de localização e menor que $\bar{\varepsilon}$ na zona elástica. Da equação 3.16 conclui-se que $\tilde{\nabla}^{2}<0$, ou seja, velocidade imaginária. Portanto, a única alternativa possível é que a interface não se move $(\widetilde{\nabla}=0)$ e a zona de localização permanece infinitamente pequena com $\mathrm{s} \rightarrow 0 . \mathrm{Da}$ equação 3.16 tem-se $\sigma^{\mathrm{s}}=\sigma^{\mathrm{e}}$, ou seja, tensão contínua em $\mathrm{x}=\mathrm{s}$, embora as deformações sejam descontínuas.

Portanto, supondo $\mathrm{S} \rightarrow 0$ (que resulta em $\varepsilon^{s} \rightarrow \infty$ ), obtém-se uma expressão para $\varphi(\mu)$ :

$\varphi(\mu)=-\mathrm{v} \mu=-\mathrm{v}\left\langle\mathrm{t}+\frac{\mathrm{x}-\mathrm{L}}{\mathrm{C}_{\mathrm{e}}}\right)$ 
e as expressões completas para ue $\varepsilon$ para $0 \leq \mathrm{t} \leq 2 \mathrm{~L} / \mathrm{c}_{\mathrm{e}}$ e $\mathrm{x}<0$ :

$$
\begin{aligned}
& \mathrm{u}=-\mathrm{v}\left(\mathrm{t}-\frac{\mathrm{L}+\mathrm{x}}{\mathrm{C}_{\mathrm{e}}}\right)-\mathrm{v}\left(\mathrm{t}-\frac{\mathrm{L}-\mathrm{x}}{\mathrm{C}_{\mathrm{e}}}\right) \\
& \varepsilon=\frac{\mathrm{v}}{\mathrm{C}_{\mathrm{e}}}\left[\mathrm{H}\left(\mathrm{t}-\frac{\mathrm{L}+\mathrm{x}}{\mathrm{C}_{\mathrm{e}}}\right)-\mathrm{H}\left(\mathrm{t}-\frac{\mathrm{L}-\mathrm{x}}{\mathrm{C}_{\mathrm{e}}}\right)\right]
\end{aligned}
$$

Para o lado direito da barra, aplica-se uma solução simétrica. Consequentemente:

$$
\begin{array}{ll}
u=-2 v\left(t-\frac{L}{C_{e}}\right), & x \rightarrow 0^{-} \\
u=2 v\left(t-\frac{L}{C_{e}}\right), & x \rightarrow 0^{+}
\end{array}
$$

tem-se um salto nos deslocamentos em $\mathrm{x}=0$ para $\mathrm{t}>\mathrm{t}$ de dimensão:

$$
[[\mathrm{u}]]=4 \mathrm{v}\langle\mathrm{t}-\hat{\mathrm{t}}\rangle
$$

e, portanto, a deformação:

$$
\begin{array}{r}
\varepsilon=\frac{\mathrm{v}}{\mathrm{C}_{\mathrm{e}}}\left[\mathrm{H}\left(\mathrm{t}-\frac{\mathrm{L}+\mathrm{x}}{\mathrm{C}_{\mathrm{e}}}\right)-\mathrm{H}\left(\mathrm{t}-\frac{\mathrm{L}-\mathrm{x}}{\mathrm{C}_{\mathrm{e}}}\right)+4\right\rangle \mathrm{C}_{\mathrm{e}} \mathrm{t}-\mathrm{L}\langle\delta(\mathrm{x})] \\
\mathrm{x} \leq 0, \quad 0 \leq \mathrm{t} \leq \frac{2 \mathrm{~L}}{\mathrm{C}_{\mathrm{e}}}
\end{array}
$$

onde $\delta(\mathrm{x})$ é a função delta de Dirac.

A tensão é calculada como:

$\sigma=\frac{\mathrm{EV}}{\mathrm{C}_{\mathrm{e}}}\left[\mathrm{H}\left(\mathrm{t}-\frac{\mathrm{L}+\mathrm{x}}{\mathrm{C}_{\mathrm{e}}}\right)-\mathrm{H}\left(\mathrm{t}-\frac{\mathrm{L}-\mathrm{x}}{\mathrm{C}_{\mathrm{e}}}\right)\right]$

A tensão em $\mathrm{x}=0$ é nula e, portanto, o ponto se comporta como um contorno livre. Conforme Adhenbad ${ }^{46}$, em um contorno livre, a onda se reflete como um 
pulso com tensão inversa à tensão do pulso incidente. Interação entre a zona sottening e a zona dástica, passa a ser caracterizada pela descontinuidade nos campos de deslocamento e tensões.

\subsection{Consideração de gradientes de ordem superior}

Como já foi dito, um método de regularização para os modelos com encruamento negativo consiste na introdução de gradientes de ordem superior na função de escoamento ou na relação constitutiva tensão- defomaçãa Neste trabalho, limita-se a estudar o caso em que as alterações são feitas na função de plasticidade, de modo que a mesma passa a depender do parâmetro $\lambda$ e de seus gradientes de ordem superior:

$$
\mathrm{f}\left(\sigma, \lambda, \nabla^{2} \lambda, \nabla^{4} \lambda\right)=\sigma-\mathrm{k} \lambda+\mathrm{G} \nabla^{2} \lambda-\mathrm{G}_{2} \nabla^{4} \lambda
$$

$\mathrm{Na}$ relação anterior, $\mathrm{C}_{\mathrm{i}}=\mathrm{c}_{\mathrm{i}}[\lambda] \quad(\mathrm{i}=1,2)$ são chamados parâmtros de difusão e intrinsecamente envolvem compimentos intemos relacionados à banda de localização ( Miiblhaus \& Aifantis ${ }^{7}$ ).

Os sinais dos termos com gradiente garantem a hiperbolicidade do modelo conforme demonstra o Anexo A.

Limitando-se ainda ao exemplo simples utilizado no item anterior, porém, agora com encruamento negativo linear (h constante) para melhor visualização do problema, a 3.26 assume a forma:

$$
\mathrm{f}=\sigma-\bar{\sigma}-\mathrm{h}^{\mathrm{p}}+\mathrm{G}_{1}{ }^{2} \mathrm{p}^{\mathrm{p}}-\mathrm{C}_{2} \nabla_{\varepsilon}^{4} \mathrm{p}
$$

e a equação governante do problema passa a ser dada por:

$$
\mathrm{G}\left(\frac{\partial^{4} u}{\partial x^{4}}-\frac{1}{c_{e}^{2}} \frac{\partial^{4} u}{\partial x^{2} \partial t^{2}}\right)-G_{2}\left(\frac{\partial^{6} u}{\partial x^{6}}-\frac{1}{c_{e}^{2}} \frac{\partial^{6} u}{\partial x^{4} \partial t^{2}}\right)-\frac{E+h}{c_{e}^{2}} \frac{\partial^{2} u}{\partial t^{2}}+h \frac{\partial^{2} u}{\partial x^{2}}=0
$$

Considerando-se a propagação de uma onda harmônica simples de amplitude A,

$$
u(x, t)=A e^{i(q x-\omega t)}
$$


e supondo, primeiramente, que a onda não ultrapasse seu limite elástico, substituindo-se 3.29 na equação de onda elástica 3.3, resulta:

$\mathrm{C}_{\mathrm{f}}=\frac{\omega}{\mathrm{q}}=\mathrm{C}_{\mathrm{e}}$

percebe-se que a velocidade de fase $\mathrm{c}_{\mathrm{f}}$ é uma constante independente do número de onda q Significa que ondas curtas se propagam com a mesma velocidade que ondas longas, e que o meio é, nessas condições, dito nãodspersiva Caso $\mathrm{c}_{\mathrm{f}}$ não seja constante, mas dependente de q diz-se que o meio é dispesiva Dispersão é um fenômeno importante porque governa a mudança da forma de um pulso durante sua propagação através do meio (Graff ${ }^{47}$ ). Como 0 meio é homogêneo e está em regime elástico, a velocidade de fase é a própria velocidade de propagação elástica.

A equação diferencial 3.28 não apresenta mais a forma de uma equação de onda simples de modo que uma solução na forma $g(x \pm d)$, portanto, pode não mais satisfaze-la. Além disso, uma solução exata do problema, como anteriormente para modelo local, se torna inviável.

Uma vez que a principal característica da solução $\mathrm{g}(\mathrm{x} \pm \mathbb{\mathrm { t }})$ é a propagação de um pulso sem distorção, para um sistema governado pela equação 3.28, espera-se que algum tipo de distorção ocorra.

O primeiro passo é determinar as condições necessárias para propagação de ondas harmônicas. Ou seja, as condições em que a solução 3.29 satisfaz 3.28. Substituindo-se 3.29 em 3.28, tem-se que:

$c_{f}=\frac{\omega}{q}=c_{e} \sqrt{\frac{h+G_{1} q^{2}+c_{2} q^{4}}{E+h+G_{1} q^{2}+c_{2} q^{4}}}$

ou $\mathrm{C}_{\mathrm{f}}=\mathrm{C}_{\mathrm{f}}(\mathrm{q})$. Portanto, uma onda harmônica de freqüência $\omega$ tem sua velocidade de propagação $\mathrm{C}_{\mathrm{f}}$ governada pelo número de onda $\mathrm{q}$

Supondo um pulso no tempo $\mathrm{t}=\mathrm{t}_{0}$ formado pela superposição de ondas harmônicas, com o avanço do tempo, cada componente passa a se propagar na sua própria velocidade $\mathrm{c}_{\mathrm{f}}$, de maneira que a forma do pulso se torna cada vez mais distorcida. 


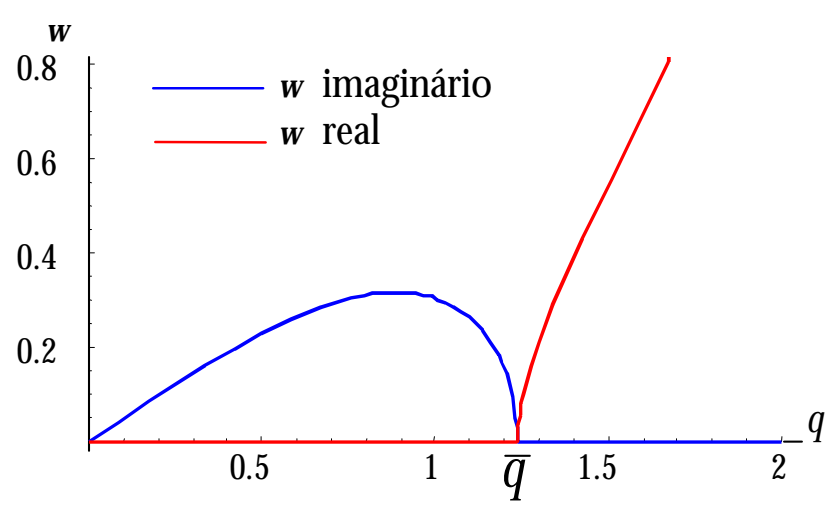

Figura 3.4. Spectrum de freqüência para modelo de plasticidade linear com gradientes segundo e quarto.

Existem valores de número de onda q para os quais se tem velocidade de fase nula ou imaginária, o que corresponde à uma perturbação que não se propaga no espaço. D ois gráficos típicos ilustram o problema: o spetrum de freqüênaia (Figura 3.4) e a arva dedispesão(Figura 3.5).

A Figura 3.4 mostra uma velocidade imaginária para valores de q menores que q. Em $\mathrm{q}=\mathrm{q}$ tem-se 0 valor limite (velocidade nula), a partir do qual a velocidade se torna real.

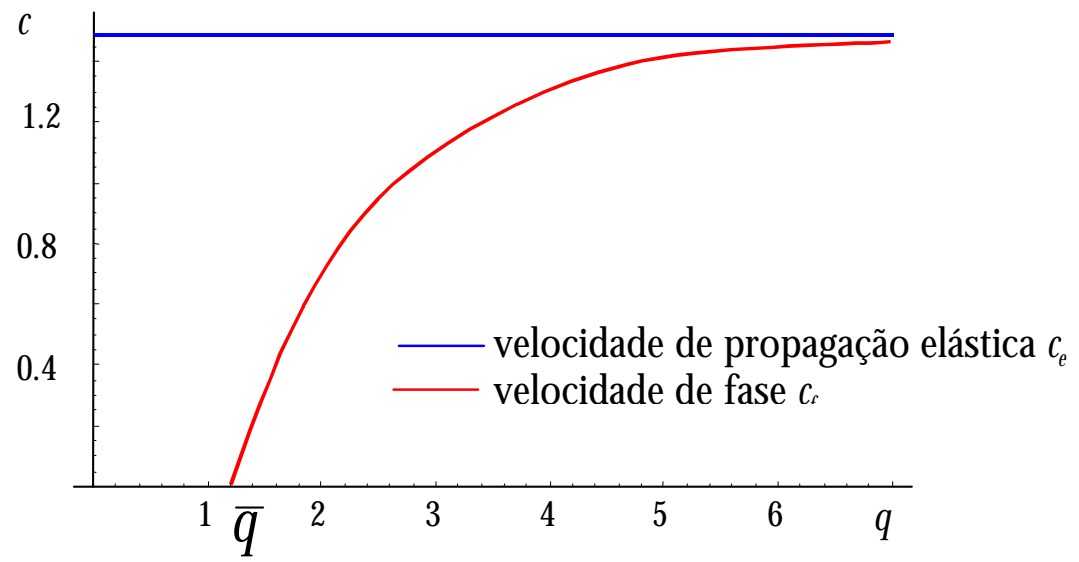

Figura 3.5. Curva de dispersão.

Conforme a Figura 3.5, para altos números de onda, que correspondem a curtos comprimentos de onda $\left(\lambda^{*}\right)$ e altas freqüências $\left(f^{*}\right)$, os valores da velocidade de fase se aproximam dos valores da velocidade de propagação elástica, que correspondem ao caso sem dispersão, indicando que 0 efeito da plasticidade é mínimo. Entretanto, para números de onda muito baixos $(\mathrm{q} \rightarrow 0)$, correspondentes a $\lambda^{*} \rightarrow \infty$ e $\mathrm{f}^{*} \rightarrow 0$, a velocidade de fase diminui rapidamente $\mathrm{c}_{\mathrm{f}} \rightarrow-\infty$. 
Considerando-se, portanto, o valor limite como aquele que zera o numerador da equação 3.30, tem-se as seguintes relações para números de onda que garantem velocidades de fase reais:

$$
\begin{array}{ll}
\mathrm{q} \geq \mathrm{q}=\sqrt{-\frac{\mathrm{h}}{\mathrm{q}}} & \text { para } \mathrm{q}>0 \text { e } \mathrm{c}_{2}=0 \\
\mathrm{q} \geq \mathrm{q}=\sqrt{\frac{-\mathrm{G}+\sqrt{\mathrm{q}^{2}-4 \mathrm{c}_{2} \mathrm{~h}}}{2 \mathrm{c}_{2}}} & \text { para } \mathrm{q} \text { qualquer e } \mathrm{c}_{2}>0
\end{array}
$$

Por outro lado, como o comprimento de onda é definido por $\lambda=\frac{2 \pi}{\mathrm{q}}$ seguem as relações:

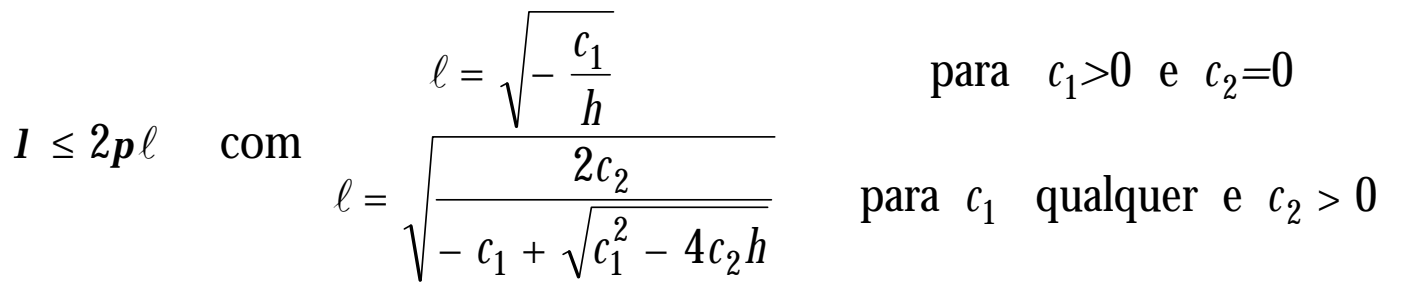

O comportamento do movimento dentro da zona de plastificação é de caráter estacionário $\left(\mathrm{C}_{\mathrm{f}}=0\right)$, pois pontos fora desta zona não recebem resposta plástica. A zona de plastificação, portanto, apresenta extensão coincidente com um comprimento de onda $\lambda=2 \pi \ell$. A região satteningé limitada pelo número de onda q. O ndas com número de onda maior não ocorrem pois provocariam paturbaçãona zona elástica.

Em continuação ao estudo da largura da banda de localização, considera-se a análise da barra ilustrada na Figura 3.6.

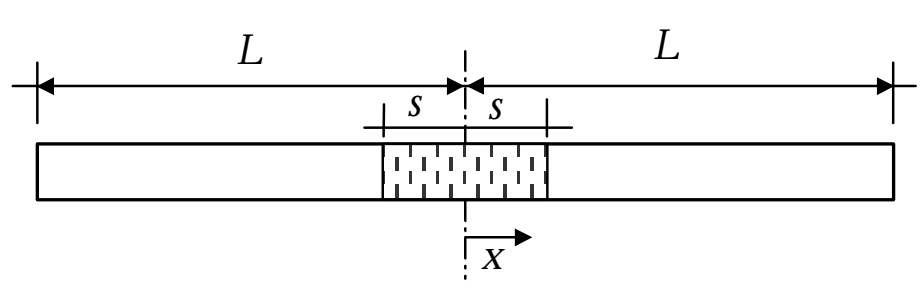

(a)

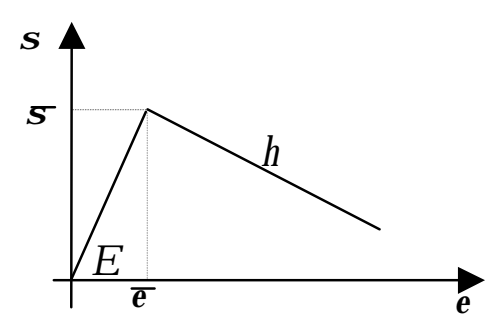

(b)

Figura 3.6.a) Barra analisada, b)lei constitutiva com encruamento negativo. 
Conforme Figura 3.6, admite-se que a deformação plástica esteja localizada em uma largura 2s simétrica em relação ao centro da barra. Da condição $\mathrm{f}=0$ tem-se a seguinte equação:

$\sigma=\bar{\sigma}+\mathrm{h}^{\mathrm{p}}-\mathrm{G}^{\varepsilon}, \mathrm{xx}+\mathrm{C}_{2} \varepsilon, \mathrm{xxxx}$

cuja integração fornece a deformação plástica de acordo com o modelo elasto plástico com gradiente de quarta ordem.

Considerando-se, primeiramente, somente 0 gradiente segundo $\left(\mathrm{c}_{2}=0\right)$ e as condições de contorno $\varepsilon^{\mathrm{p}}=0$ para $\mathrm{X}= \pm \mathrm{S}$, recuperam-se resultados já obtidos por deBarst \& Mühlhaus ${ }^{48}$. A solução da equação (3.34) é:

$\varepsilon \mathrm{p}=\frac{\sigma-\bar{\sigma}}{\mathrm{h}}\left[1-\frac{\cos \left(\mathrm{x} / \ell_{1}\right)}{\cos \left(\mathrm{s} / \ell_{1}\right)}\right]$

onde:

$\ell_{1}=\sqrt{-\frac{\mathrm{G}}{\mathrm{h}}}$

O deslocamento da barra na extremidade direita pode ser calculado como:

$\mathrm{u}=\int_{0}^{\mathrm{L}} \varepsilon \mathrm{e} \mathrm{dx}+\int_{0}^{\mathrm{s}} \varepsilon \mathrm{p} \mathrm{dx}$

Substituindo-se a equação 3.35 e a definição de $\varepsilon^{e}=\sigma / E$ na equação 3.37 , e dividindo-se por L, tem-se o valor da deformação média da barra:

$\widehat{\varepsilon}=\frac{\widehat{u}}{\mathrm{~L}}=\frac{\sigma}{\mathrm{E}}+\frac{\sigma-\bar{\sigma}}{\mathrm{Lh}}\left[\mathrm{s}-\ell_{1} \tan \left(\mathrm{s} / \ell_{1}\right)\right]$

Considerando-se a solução crítica como aquela que dissipa a menor energia, o que equivale a minimizar $\hat{\varepsilon}$ com s vale a condição:

$\frac{\mathrm{d} \varepsilon}{\mathrm{ds}}=0$

Portanto: 


$$
\cos ^{2}\left(\mathrm{~s} / \ell_{1}\right)=1
$$

D e modo que resultam valores explícitos para a largura da banda de localização s

$$
\mathrm{S}=\mathrm{n} \pi \ell_{1} \quad \mathrm{n}=1,2,3, \ldots
$$

Nota-se que para $\mathbf{s}=1$, 2s coincide com 0 valor de $\lambda$ dado pela 3.33a. Substituindo-se a equação 3.41 para $n=1$ nas equações 3.35 e 3.38 tem-se que:

$$
\begin{aligned}
& \varepsilon^{\mathrm{p}}=\frac{\sigma-\bar{\sigma}}{\mathrm{h}}\left[1+\cos \left(\mathrm{x} / \ell_{1}\right)\right] \\
& \hat{\varepsilon}=\frac{\sigma}{\mathrm{E}}+\frac{\sigma-\bar{\sigma}}{\mathrm{h}} \frac{\pi \ell_{1}}{\mathrm{~L}}
\end{aligned}
$$

Levando-se em conta o gradiente de quarta ordem no modelo, primeiramente, deve-se considerar novas condições de contorno: $\varepsilon^{p}=0$ e $\varepsilon_{, x}^{p}=0$ para $x= \pm s$. Esta última condição é compatível com uma implementação numérica, que requer continuidade na derivada das deformações plásticas. A solução da equação 3.34 é:

$\varepsilon^{\mathrm{p}}=\frac{\sigma-\bar{\sigma}}{\mathrm{h}}\left\{1-\frac{\mathrm{ae}^{\mathrm{bs}}\left(\mathrm{e}^{\mathrm{bx}}+\mathrm{e}^{-\mathrm{bx}}\right) \operatorname{sen}(\mathrm{as})-\mathrm{b}\left(1-\mathrm{e}^{2 \mathrm{bs}}\right) \cos (\mathrm{ax})}{\mathrm{asen}(\mathrm{as})\left(1+\mathrm{e}^{2 \mathrm{bs}}\right)-\mathrm{b} \cos (\mathrm{as})\left(1-\mathrm{e}^{2 \mathrm{bs}}\right)}\right\}$

onde:

$$
\begin{aligned}
& \mathrm{a}=\frac{\sqrt{-\mathrm{c}_{1}+\sqrt{\mathrm{q}^{2}-4 \mathrm{~h}_{2}}}}{\sqrt{2 \mathrm{c}_{2}}} \\
& \mathrm{~b}=\frac{\sqrt{\mathrm{c}_{1}+\sqrt{\mathrm{q}^{2}-4 \mathrm{~h}_{2}}}}{\sqrt{2 \mathrm{c}_{2}}}
\end{aligned}
$$

0 deslocamento da barra na extremidade direita pode ser calculado como pela equação 3.37. Novamente, substituindo-se a equação 3.44 e a definição de $\varepsilon^{e}=\sigma / E$ na equação 3.37, e dividindo-se por L, tem-se 0 valor da deformação média da barra na extremidade direita: 
$\hat{\varepsilon}=\frac{\sigma}{E}+\frac{\sigma-\bar{\sigma}}{L h}\left[s-\frac{\operatorname{sen}(a s)\left(1-e^{2 b s}\right)\left(a^{2}+b^{2}\right)}{a^{2} b \operatorname{sen}(a s)\left(1+e^{2 b s}\right)-a b^{2} \cos (a s)\left(1-e^{2 b s}\right)}\right]$

Considerando-se a solução crítica como aquela que dissipa a menor energia, ou seja, aquela que reproduz a curva sotteningmais acentuada do diagrama $\sigma-\widehat{\varepsilon}$ (equação 3.39), tem-se que:

$$
-\frac{\sigma-\bar{\sigma}}{\mathrm{Lh}}\left[\frac{\mathrm{a}\left(1-\mathrm{e}^{2 \mathrm{bs}}\right) \cos (\mathrm{as})+\mathrm{b}\left(1+\mathrm{e}^{2 \mathrm{bs}}\right) \operatorname{sen}(\mathrm{as})}{\mathrm{a}\left(1+\mathrm{e}^{2 \mathrm{bs}}\right) \operatorname{sen}(\mathrm{as})-\mathrm{b}\left(1-\mathrm{e}^{2 \mathrm{bs}}\right) \cos (\mathrm{as})}\right]^{2}=0
$$

A Figura 3.7 mostra a equação 3.47, adimensionalizada pelo termo $\mathrm{Lh}(\sigma-\bar{\sigma})$, em função da largura de banda s para valores de a e bquaisquer. Percebe-se que a raiz não é única.

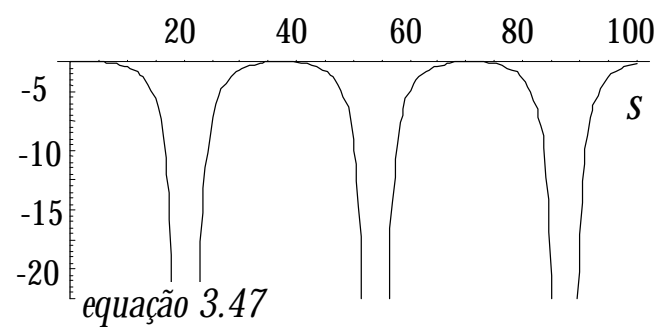

Figura 3.7. Equação 3.47(y) em função da largura de banda s (x).

De modo a obter um valor explícito para a largura da banda de localização w foi feitas a seguinte simplificação:

$\mathrm{e}^{2 \mathrm{bs}}>>>1$

Portanto:

$\mathrm{s}=\frac{1}{\mathrm{a}}\left[ \pm \mathrm{n} \pi+\arctan \left(\frac{\mathrm{a}}{\mathrm{b}}\right)\right] \quad \mathrm{n}=1,2,3 \ldots$

Convém observar que a equação acima, que representa a largura da banda via um exemplo resolvido analiticamente, apresenta 0 termo $\arctan (\mathrm{a} / \mathrm{b}) / \mathrm{a}$, que não aparece na equação 3.33 (largura de banda calculada através do estudo de propagação de onda). Apesar da imposição inicial da condição de contorno homogênea $\varepsilon_{, \mathrm{x}}^{\mathrm{p}}=0$ para $\mathrm{x}= \pm \mathrm{S}$ na resolução analítica, encontrou-se 0 mesmo 
valor quando não impõe-se esta condição inicialmente, deixando a solução em função de constantes. Estas constantes se anulam e o resultado coincide com 3.49.

Substituindo-se a equação 3.49 para $\mathrm{n}=1$ nas equações 3.44 e 3.46 e fazendo-se as devidas simplificações (conforme 3.48) tem-se que:

$$
\begin{aligned}
& { }_{\varepsilon} \mathrm{p}=\frac{\sigma-\sigma_{\mathrm{y}}}{\mathrm{h}}\left\{1-\frac{\mathrm{a}^{2} \mathrm{e}^{-\mathrm{b} / \mathrm{a}[\pi+\arctan (\mathrm{a} / \mathrm{b})]}\left(\mathrm{e}^{\mathrm{bx}}+\mathrm{e}^{-\mathrm{bx}}\right)-\mathrm{b} \sqrt{\mathrm{a}^{2}+\mathrm{b}^{2}} \cos (\mathrm{ax})}{\mathrm{a}^{2}+\mathrm{b}^{2}}\right\} \\
& \hat{\varepsilon}=\frac{\sigma}{\mathrm{E}}+\frac{\sigma-\sigma_{\mathrm{y}}}{\mathrm{Lh}}\left[\frac{\mathrm{b}(\pi+\arctan \mathrm{a} / \mathrm{b})-\mathrm{a}\rceil}{\mathrm{ab}}\right]
\end{aligned}
$$

A Figura 3.8 ilustra a deformação plástica (definida pela equação 3.50) ao longo da barra para vários níveis de tensão.

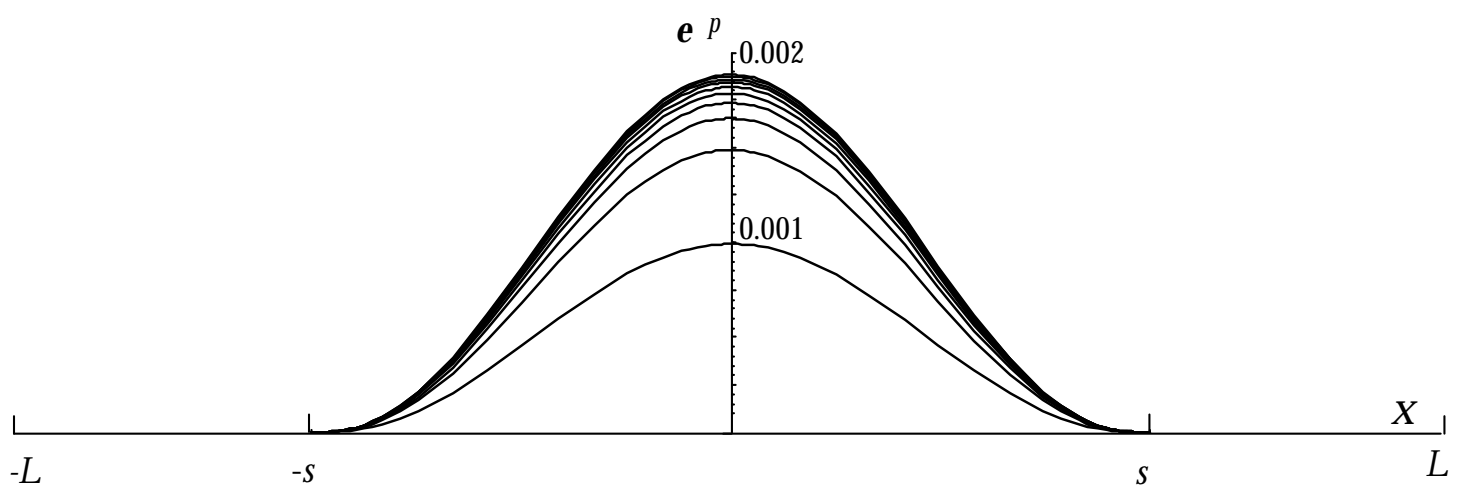

Figura 3.8. Distribuição de deformação plástica ao longo da barra.

D efinindo-se a largura scomo as segunda e terceira raízes da equação 3.49, tem-se, como mostra a Figura 3.9, duas e três zonas de localização, respectivamente. Podese simular numericamente a existência de múltiplas zonas de localização.

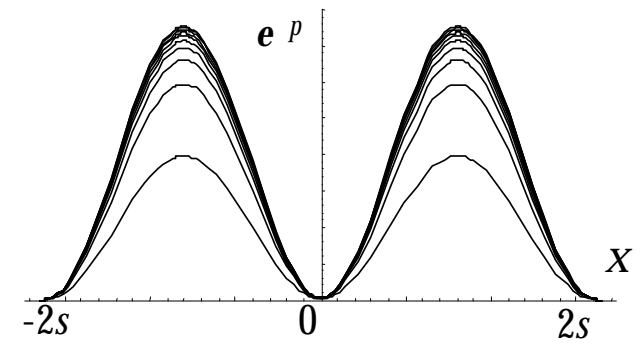

(a)

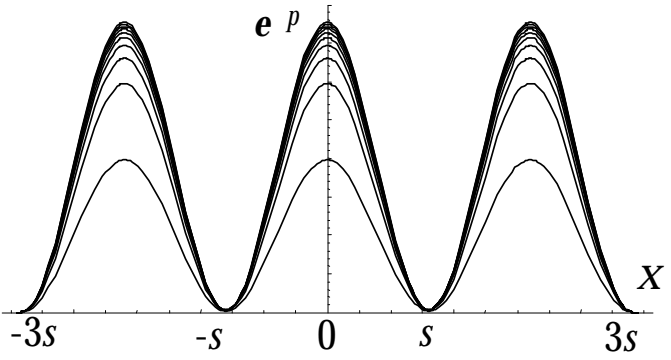

(b)

Figura 3.9. Distribuição de deformação plástica ao longo da barra, para largura de banda w relativas à(a) segunda ou (b) quarta raíz da equação 3.49. 
Para uma distribuição de deformação plástica ao longo da barra definida através das equações 3.45 e 3.50, a Figura 3.10 mostra a influência de cada parâmetro de difusão $\left(\mathrm{G}, \mathrm{G}_{2}\right)$ individualmente. As Figuras 3.10a,b mostram a influência do parâmetro G (variando linearmente de 0 a 200), quando $\mathrm{G}_{2}=0$ e quando $\mathrm{G}_{2}$ é constante e igual a 100, respectivamente. Percebe-se que 0 parâmetro q tem influência direta na determinação da largura de banda.

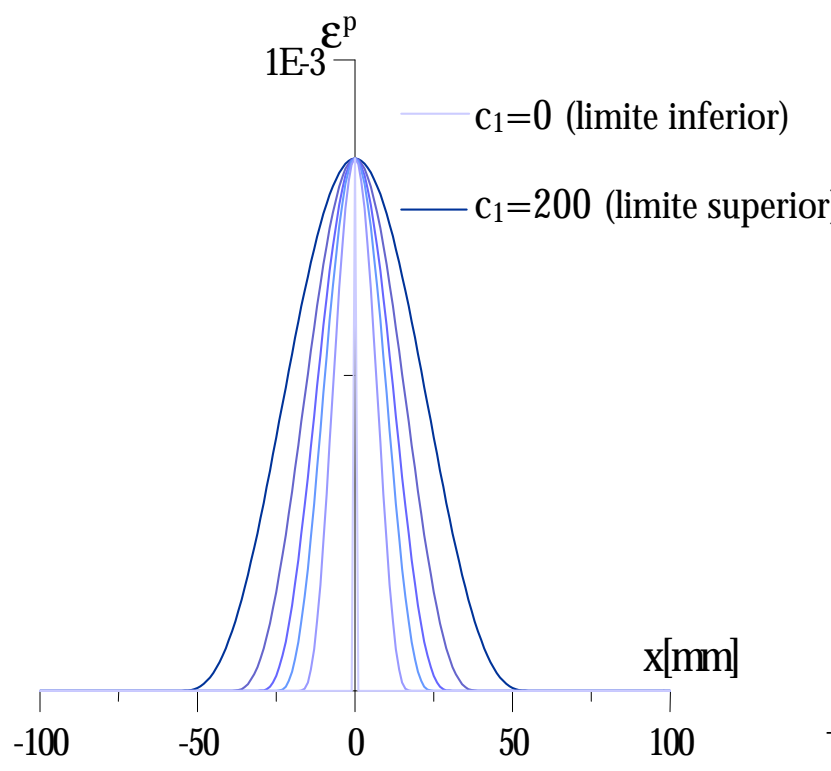

(a)

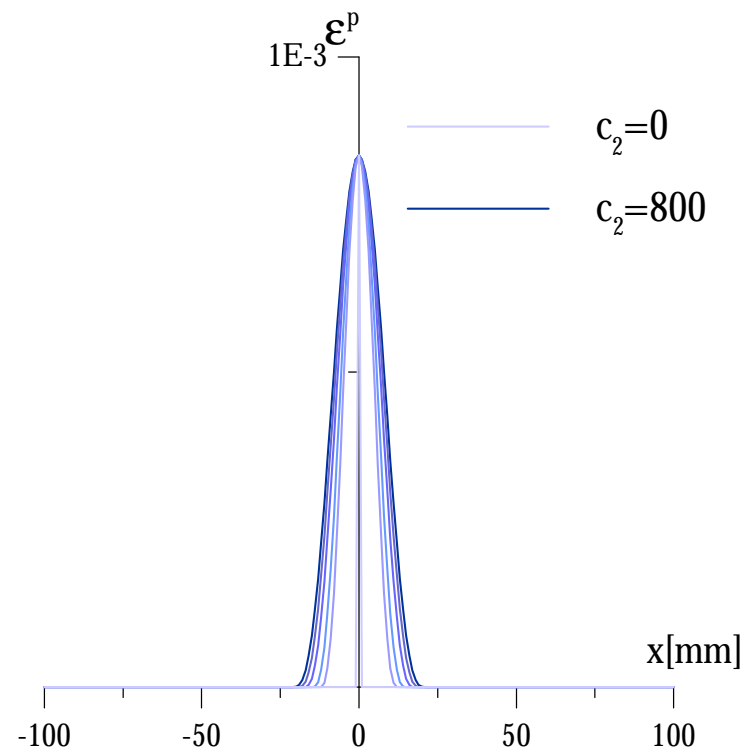

(c)

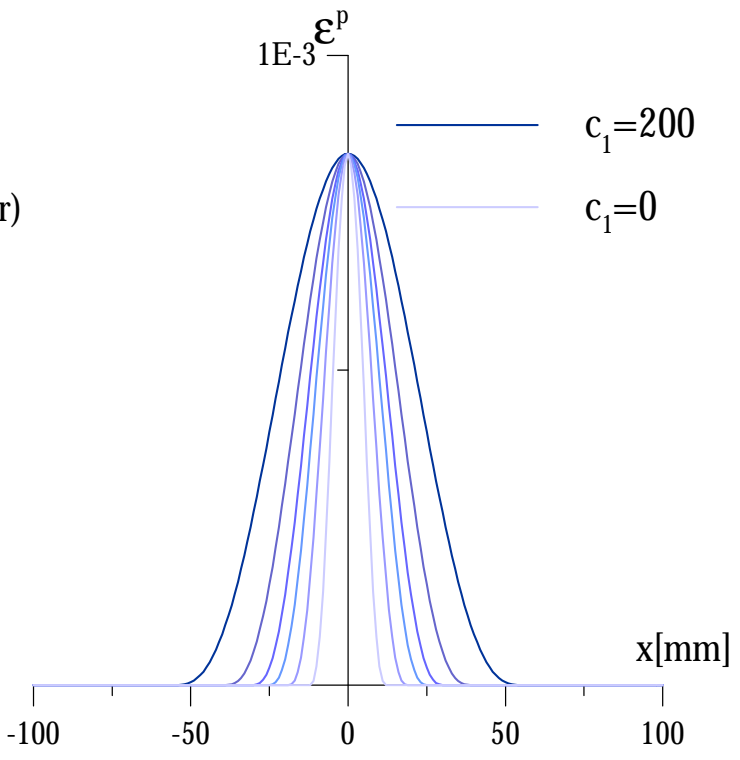

(b)

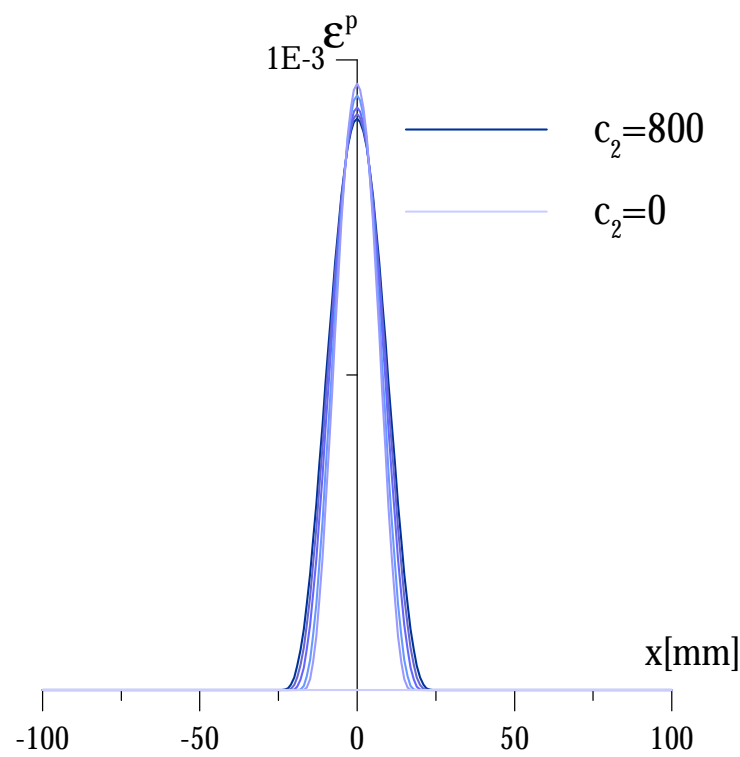

(d)

Figura 3.10. Influência de $c_{1}$ quando (a) $c_{2}=0$ e quando (b) $c_{2}$ é constante e igual a 100. Influência de $c_{2}$ quando (c) $c_{1}=0$ e quando (d) $c_{1}$ é constante e igual a 100. 
As Figuras 3.10c,d mostram a influência de $G_{2}$ (variando entre 0 e 800), quando $\mathrm{G}=0$ e quando $\mathrm{G}$ é constante e igual a 100, respectivamente. Os resultados mostram que $\mathrm{G}_{2}$ deve ser maior que $\mathrm{G}$ em valor numérico para se obter a regularização numa mesma largura de banda. Essa conclusão porém, é apenas qualitativa, pois G e $\mathrm{G}_{2}$ não podem ser comparados diretamente, uma vez que tem dimensões diferentes.

O parâmetro $\mathrm{C}_{2}$ sozinho modifica pouco o pico de deformação plástica, mas para $\mathrm{G}$ constante e diferente de zero, 0 parâmetro $\mathrm{G}_{2}$ diminui 0 valor da deformação plástica conforme aumenta a largura da banda.

\subsubsection{Estudo de bandas de cisalhamento}

Com o objetivo de ressaltar algumas características dos modelos com gradiente, considera-se um corpo com deformação inicialmente homogênea, e pretende-se determinar as condições em que, em uma análise estática, as equações diferenciais admitem soluções não - homogêneas, além da solução fundamental homogênea (Zlib\& Aifantis ${ }^{16}$ e Müblhaus \& Aifantis ${ }^{7}$ ).

Para o critério de plastificação de VonMises(Mühlhaus \& Aifantis ${ }^{7}$ ):

$$
\begin{aligned}
\dot{\lambda} & =\sqrt{2 \dot{\varepsilon}^{\mathrm{p}} \cdot \dot{\varepsilon}^{\mathrm{p}}} \\
\tau & =\sqrt{\mathrm{J}_{2}}
\end{aligned}
$$

com $\mathrm{J}_{2} \mathrm{o}$ segundo invariante da parte desviadora do tensor de tensões. A primeira equação refere-se à hipótese de encruamento por deformação e $\tau$ é a tensão equivalente.

A equação 3.1e pode ser rescrita na forma:

$$
\left.f=\sqrt{\mathrm{J}_{2}}-\mathrm{g} \dot{\lambda}\right]
$$

Conforme 3.1.c, a deformação pode ser dividida em uma parcela elástica e outra plástica. Substituindo-se 3.1d em 3.1c expressa em taxas:

$$
\dot{\varepsilon}=\mathrm{E}^{-1} \dot{\sigma}+\lambda \frac{\sigma^{\mathrm{d}}}{2 \tau}
$$


onde $\sigma^{\mathrm{d}}=\sigma-\sigma_{\mathrm{m}} \mathrm{I}$ é a parte desviadora do tensor de tensões, com $\sigma_{\mathrm{m}}=\left(\sigma_{1}+\sigma_{2}+\sigma_{3}\right) / 3$ a tensão hidrostática, onde os índices 1,2 e 3 indicam a direção no espaço tridimensional.

Por simplificação, despreza-se a compressibilidade elástica do material e considerase 0 caso de deformação plana, $\sigma_{3}=1 / 2 \sigma_{1}$ e $\sigma_{2}=0$.

A taxa de deformação pode ser escrita na forma:

$$
\dot{\varepsilon}_{1}=\frac{(1-v)}{\mathrm{G}} \dot{\sigma}_{1}^{\mathrm{d}} \quad \dot{\varepsilon}_{2}=\frac{v}{\mathrm{G}} \dot{\sigma}_{2}^{\mathrm{d}}
$$

onde $\mathrm{G}=\frac{E}{2(1+v)}$ é o módulo de elasticidade transversal. A deformação plástica, conforme a segunda parcela de 3.54:

$\dot{\varepsilon_{1}}=\frac{1}{2} \lambda \quad \dot{\varepsilon_{2}}=-\frac{1}{2} \lambda \dot{ }$

D este modo, para $v=1 / 2$ :

$$
\begin{aligned}
& \dot{\sigma}_{1}^{\mathrm{d}}=2 \mathrm{G}\left(\dot{\varepsilon}_{1}-\frac{1}{2} \dot{\lambda}\right) \\
& \dot{\sigma}_{2}^{\mathrm{d}}=2 \mathrm{G}\left(\dot{\varepsilon}_{2}+\frac{1}{2} \dot{\lambda}\right) \\
& \dot{\sigma_{12}}=2 \dot{\mathrm{r}}_{12}
\end{aligned}
$$

Substituindo-se 3.57-3.59 na condição de consistência $\mathrm{f}=0$, tem-se que:

$$
2 \mathrm{G}_{1}=(\mathrm{G}+\mathrm{h}) \dot{\lambda}-\mathrm{G} \nabla^{2} \dot{\lambda}+\mathrm{G}_{2} \nabla^{4} \dot{\lambda}
$$

Supõe-se a existência de soluções particulares do tipo:

$$
\dot{\lambda}=\dot{\lambda_{0}} \mathrm{e}^{(\mathrm{iqg})} \quad \dot{\mathrm{u}}=\dot{\mathrm{u}}_{0} \mathrm{e}^{(\mathrm{iqg})}
$$

onde: 
$\mathrm{y}=-(\sin \theta) \mathrm{x}_{1}+(\cos \theta) \mathrm{x}_{2}$

onde qé o número de onda. 0 plano ycontém a banda de localização, $\theta$ é 0 ângulo entre a normal de ye a direção $\mathbf{x}_{2}$.

Pode-se definir:

$\dot{\mathrm{u}}_{1}=\frac{\partial \dot{\mathrm{u}}}{\partial \mathrm{x}_{2}}=\dot{\mathrm{u}}_{0} \mathrm{e}^{(\mathrm{iqg})} \cos \theta \quad \dot{\mathrm{u}}_{2}=\frac{\partial \dot{\mathrm{u}}}{\partial \mathrm{x}_{1}}=-\dot{\mathrm{u}}_{0} \mathrm{e}^{(\mathrm{iqg})} \operatorname{sen} \theta$

Substitui-se 3.57-3.59 nas condições de equilíbrio estático, e aplica-se 3.61-3.63 à soma das equações resultantes. Finalmente, tem-se que:

$\dot{\lambda}_{0}(\operatorname{sen} 2 \theta)+\dot{u}_{0} \mathrm{q}^{2}=0$

Substituindo-se 3.61-3.63 em 3.60:

$-\mathrm{u}_{0} G q^{2}(\operatorname{sen} 2 \theta)+\left(h+G+G q^{2}+G_{2} q^{4}\right) \dot{\lambda_{0}}=0$

Com as equações 3.64-3.65 pode-se escrever o seguinte sistema homogêneo de equações:

$\left[\begin{array}{cc}\mathrm{q}^{2} \operatorname{sen} 2 \theta & \mathrm{q}^{4} \\ \frac{\left(\mathrm{h}+\mathrm{G}+\mathrm{G}^{2} \mathrm{q}^{2}+\mathrm{G}_{2} \mathrm{q}^{4}\right)}{\mathrm{G}} & \mathrm{q}^{2} \operatorname{sen} 2 \theta\end{array}\right]\left\{\begin{array}{l}\dot{u}_{0} \\ \dot{\lambda}_{0}\end{array}\right\}=0$

E a existência de soluções não triviais leva a:

$\frac{h}{G}=\operatorname{sen}^{2} 2 \theta-1-\frac{G}{G} q^{2}-\frac{C_{2}}{G} q^{4}$

A Figura 3.11 ilustra $\frac{h}{G} \times q$ para vários valores de $\mathrm{G}_{1}$ e $\mathrm{C}_{2}$, considerando para $\theta \quad 0$ valor máximo $\theta_{\alpha}=\frac{\pi}{4}$. 
(a)

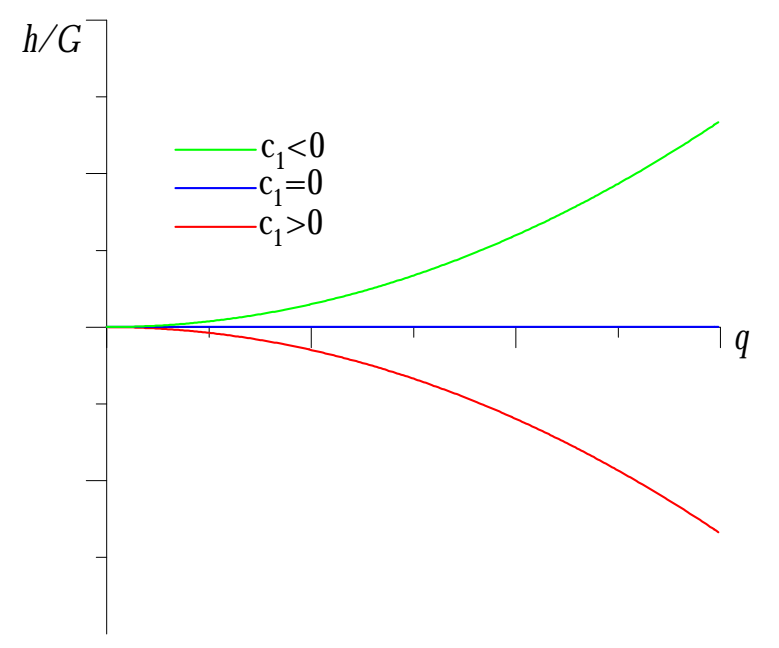

(b)

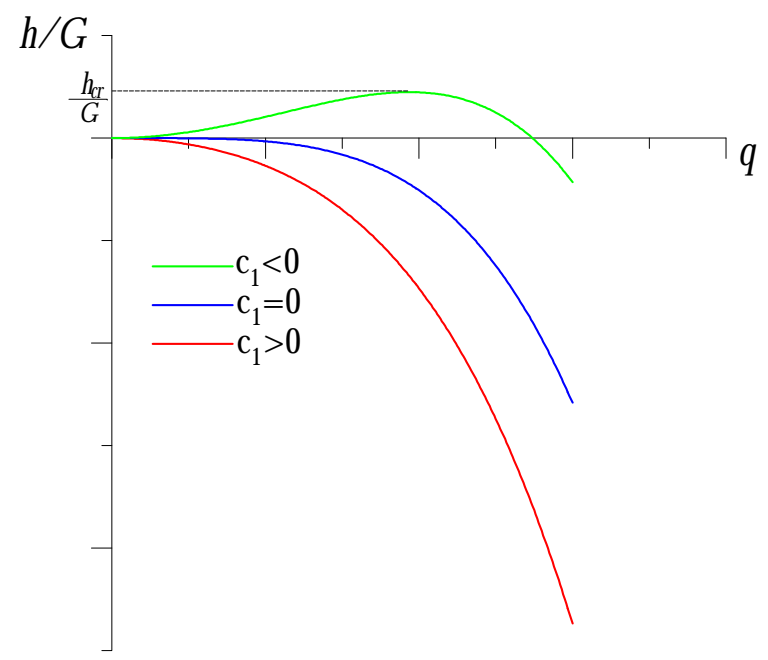

(c)

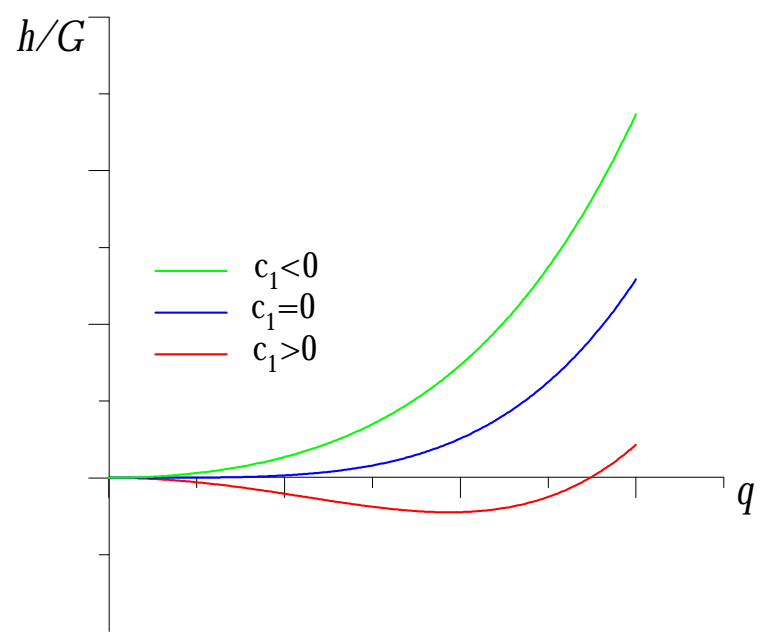

Figura 3.11. Valores de h/ G para (a) $\mathrm{c}_{2}=0$, (b) $\mathrm{c}_{2}>0$, (c) $\mathrm{c}_{2}<0$ 
Para $\mathrm{G}>0$ e $\mathrm{C}_{2}=0$ tem-se $\frac{\mathrm{h}}{\mathrm{G}}=\operatorname{sen}^{2}(2 \theta)-1-\frac{\mathrm{G}}{\mathrm{G}} \mathrm{q}^{2}<0$ para qualquer valor de $\mathrm{q}$ conforme mostra a Figura 3.11a. 0 valor máximo da relação $h / G$, que conduz a um mínimo de energia, obtém-se para $\mathrm{q}_{\mathrm{r}}=0$, de modo que a relação $\mathrm{h} / \mathrm{G}$ crítica é definida como:

$$
\frac{h_{r}}{G}=0
$$

Portanto, para modelos associativos locais e não locais com $\mathrm{G}_{\mathrm{S}}>0$ e $\mathrm{C}_{2}=0$, a localização ocorre a partir do pico de tensão, quando se inicia a resposta suttening

Para $\mathrm{G}_{1}<0$ e $\mathrm{C}_{2}=0$ (Figura 3.11a) tem-se $\frac{\mathrm{h}}{\mathrm{G}}=\sin ^{2}(2 \theta)-1-\frac{\mathrm{G}}{\mathrm{G}} \mathrm{q}^{2}>0$ para qualquer valor de q o que não é razoável, pois indica que não ocorrerá localização, ou resposta homogênea em softening Uma situação igualmente inaceitável seria para $\mathrm{C}_{2}<0$ e $\mathrm{G}$ qualquer, conforme mostra a Figura 3.11c.

Para $\mathrm{G}_{1}>0$ e $\mathrm{c}_{2}>0$ (Figura 3.11b), obtém-se a mesma situação de $\mathrm{G}_{1}>0$ e $\mathrm{c}_{2}=0$, com localização a partir do pico de tensão.

Uma última possibilidade ilustrada na Figura 3.11b seria para $\mathrm{G}_{1}<0$ e $\mathrm{C}_{2}>0$, onde 0 valor máximo da relação $\mathrm{h} / \mathrm{G}$ obtém-se para $\mathrm{q}_{\mathrm{rr}}=\sqrt{-\mathrm{G}_{\mathrm{g}} / 2 \mathrm{G}_{2}}$, de modo que a relação $\mathrm{h} / \mathrm{G}$ crítica é definida como:

$$
\frac{h_{r}}{G}=\frac{\mathrm{G}^{2}}{4 \mathrm{Gc}_{2}}
$$

O u seja, a localização pode, matematicamente, iniciar-se em regime hardaning $\left(h_{\mathrm{r}}>0\right)$. Nos modelos locais, este tipo de comportamento pode ocorrer somente em modelos multidissipativos ou não-associativos (Rizzi $\left.{ }^{4}\right)$. 


\section{MODELO DE DANO COM GRADIENTE}

\subsection{Introdução}

Neste capítulo, é proposta a introdução de gradientes de ordem superior em um modelo de dano local proposto por Comi $\notin$ al. ${ }^{17}$. Com uma formulação adequada em elementos finitos, objetiva-se obter resultados coerentes para problemas de análise estática e dinâmica na presença do fenômeno de localização.

\subsection{Modelo de dano}

$\mathrm{Na}$ mecânica do dano clássica, a lei constitutiva secante é descrita com a introdução de uma variável de dano $\mathrm{D}$, responsável pela perda de rigidez do material, conforme ilustra a Figura 4.1.

De grande importância para a formulação de uma relação constitutiva de um meio com dano, tratado como meio contínuo, é o estabelecimento de uma hipótese de equivalência de deformação, de tensão ou de energia. $\mathrm{O}$ u seja, o material com dano pode ser substituído por um material íntegro, desde que a deformação, a tensão ou a energia sejam substituídos por valores efetivos ou equivalentes.

Para o modelo em questão foi adotada uma hipótese de enegia equivalenteexpressa da seguinte forma: 0 trabalho virtual interno do material danificado pode ser obtido através de um material íntegro, envolvendo tensão e deformação efetiva. 


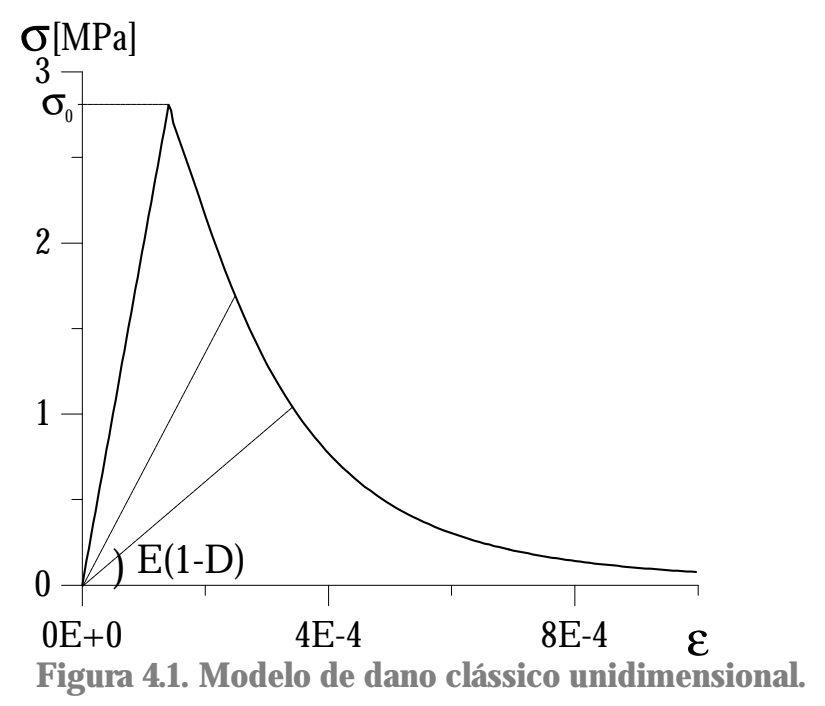

A equivalência energética fornece uma aproximação intermediária entre as hipóteses de deformação e tensão equivalente $\left(\operatorname{Rizzi}^{4}\right)$. A condição de equivalência em energia se escreve como $\varepsilon \sigma=\widetilde{\varepsilon} \widetilde{\sigma}$ e se verifica para:

$$
\begin{aligned}
& \tilde{\sigma}=\frac{\sigma}{(1-\mathrm{D})} \\
& \tilde{\varepsilon}=(1-\mathrm{D}) \varepsilon
\end{aligned}
$$

onde $\tilde{\sigma}$ e $\widetilde{\varepsilon}$ são a tensão e a deformação efetiva, respectivamente. A variável de dano varia entre 0 e $1 ; D=0$ corresponde ao estado elástico inicial, sem dano, enquanto $\mathrm{D}=1$ corresponde à ruptura do material.

Portanto, no material íntegro vale a relação , a qual substituindo-se as 4.1 e 4.2 resulta:

$\sigma=(1-\mathrm{D})^{2} \mathrm{E} \varepsilon$

A rigidez secante $E_{\mathscr{C}}$ do material danificado é então definida por:

$$
\mathrm{E}_{\mathrm{SC}}=(1-\mathrm{D})^{2} \mathrm{E}
$$

sendo E a rigidez elástica inicial.

D este modo, considera-se o seguinte modelo de dano local proposto por Comi $\&$ al. ${ }^{17}$ : 


$$
\begin{aligned}
& \psi=\frac{1}{2}(1-D)^{2}{ }^{\mathrm{T}} \mathrm{E} \varepsilon \\
& \sigma=\frac{\partial \psi}{\partial \varepsilon}=(1-\mathrm{D})^{2} \mathrm{E} \varepsilon ; \mathrm{Y}=-\frac{\partial \psi}{\partial \mathrm{D}}=(1-\mathrm{D}) \varepsilon \cdot \mathrm{E} \varepsilon \\
& \mathrm{f}=\frac{1}{2} \mathrm{Y}+\mathrm{b}_{1}\left(1+\mathrm{b}_{2} \mathrm{D}\right)(1-\mathrm{D}) \varepsilon_{\mathrm{v}}-\mathrm{b}_{3} \mathrm{D}-\mathrm{k} \leq 0 \\
& \mathrm{f} \leq 0 ; \quad \dot{\mathrm{D}} \geq 0 ; \quad \mathrm{f} \dot{\mathrm{D}}=0
\end{aligned}
$$

A equação 4.5 define a energia livre de Helmholtz como função do tensor de deformações $\varepsilon$ e da variável isótropa de dano D. Na equação $4.6 \mathrm{Y}$ é a força termodinâmica associada ao dano e interpretada como energia liberada em correspondência ao dano criado. O domínio elástico e as condições de carregamento - descarregamento são definidas nas equações 4.7 e 4.8, fé a função de carregamento; $\varepsilon_{\mathrm{V}}=\mathrm{I} \cdot \varepsilon$ é a deformação volumétrica (I é o tensor identidade de segunda ordem), b e k são parâmetros não - negativos do material.

Na equação 4.7 a parcela envolvendo a deformação volumétrica permite considerar o comportamento dissimétrico em tração e compressão, exibido em materiais como 0 concreto. 0 parâmetro $\mathrm{k}$ é uma energia inicial que define o início da evolução da variável de dano. Os parâmetros $\mathrm{b}_{1}$ e $\mathrm{b}_{3}$ permitem definir a tensão de pico, com a existência ou não de encruamento positivo, bem como a inclinação da curva em correspondência com o encruamento negativo.
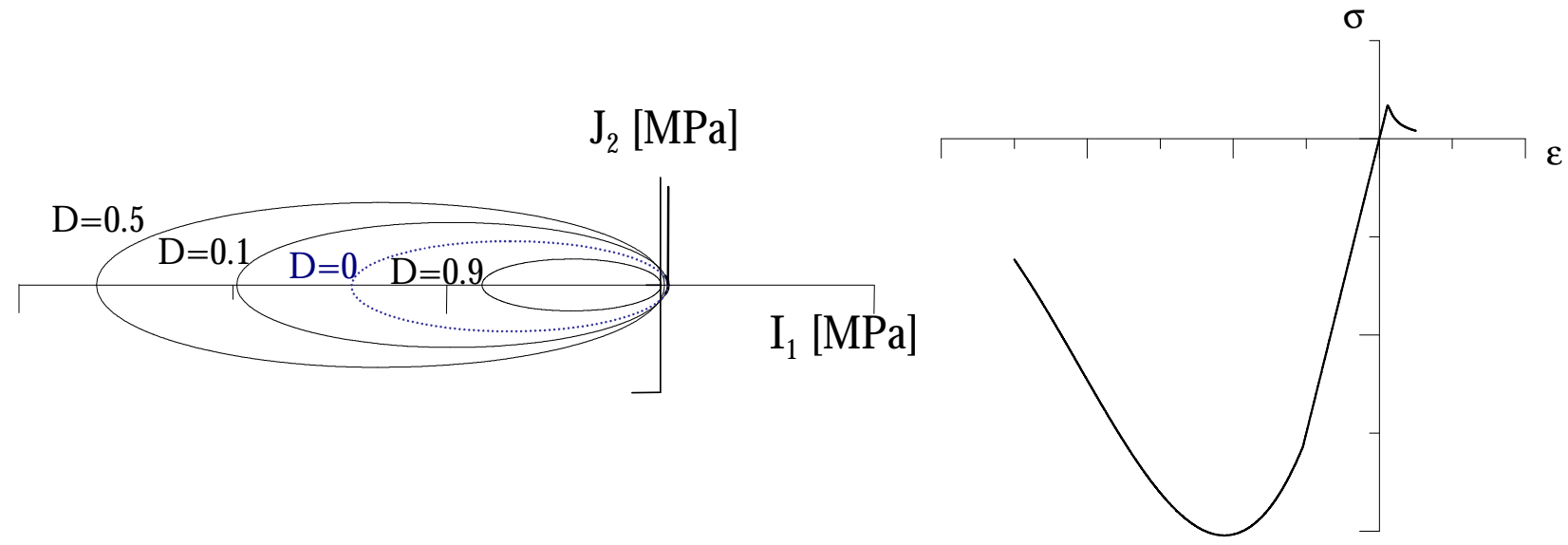

(a)

(b)

Figura 4.2 (a) Projeção do modelo na superfície de Watergaard; (b) Comportamento dissimétrico em tração e compressão permitido pelo modelo. 
A Figura 4.2 mostra a evolução de f no plano $I_{1}-\sqrt{J_{2}}$, onde $I_{1}$ é o primeiro invariante das tensões e $J_{2}$ o segundo invariante da parte desviadora $\left(\mathrm{Com}^{10}\right)$.

Esta lei constitutiva é uma simplificação da versão proposta por Comi $\notin$ al. ${ }^{17}$. Importante observar que, para o modelo dado pelas equações 4.5-4.8 o dano pode alcançar 0 valor limite $\mathrm{D}=1$ somente no caso de deformações infinitas, mas a correspondente energia de fratura (definida conforme Bazant \& Ceeblin ${ }^{36}$ ) é limitada.

Uma versão não - local do modelo pode ser obtida introduzindo-se na função critério de danificação gradientes espaciais da variável de dano $\mathrm{D}$. Considera-se a seguinte forma geral que inclui termos com 'gradientes de segunda e quarta ordem':

$$
\mathrm{f}=\mathrm{f}_{\mathrm{local}}+\frac{\mathrm{G}}{1-\mathrm{aD}} \Delta \mathrm{D}-\mathrm{C}_{2} \Delta^{2} \mathrm{D} \leq 0
$$

sendo c um parâmetro de difusão com dimensão de força, $\mathrm{c}_{2}$ outro parâmetro de difusão com dimensão de força vezes comprimento ao quadrado e a um parâmetro adimensional variando entre 0 e 1.

As parcelas não locais da equação 4.9 são aqui referidas genericamente como termos de gradientedesegunda equarta ardemda variáve intema dedamo Trata-se de uma questão de afinidade com a nomenclatura adotada em toda a bibliografia estudada (destaca-se, entre outros, Slyus ${ }^{8}$, Pamim $^{14}$, Muiblhaus \& Aifantis ${ }^{7}, \mathrm{Com}^{10}{ }^{10}$. $\mathrm{Na}$ verdade, aqueles termos envolvem o Lapladiano $(\Delta)$ e Lapladano segundo $\left(\Delta^{2}\right)$ da variáde intema dedano, respectivamente.

Três modelos diferentes, que são considerados a seguir, podem ser obtidos da equação (4.9) admitindo-se para os parâmetros $\mathrm{C}_{1}, \mathrm{c}_{2}$ e aas seguintes condições:

- Moddo $\mathrm{A}-\mathrm{C}_{2}=0, \mathrm{a}=0, \mathrm{G}_{1}>0$ - somente 0 termo com gradiente de segunda ordem está presente no modelo, coincidindo com aquele proposto por $\mathrm{Com}^{10}$;

- Moddo B - $\mathrm{C}_{2}=0, \mathrm{a}>0, \mathrm{G}_{1}>0$ - somente 0 termo com gradiente de segunda ordem está presente no modelo, mas a função de difusão $\mathrm{G} /(1-\mathrm{aD})$ cresce com 0 valor do dano. Em particular o valor $\mathrm{a}=1$ será considerado; 
- Moddo C - $\mathrm{C}_{2}>0, \mathrm{a}=0$, $\mathrm{g}$ qualquer - 0 termo com gradiente de quarta ordem também é considerado. Os dois parâmetros de difusão são constantes.

A presença de termos em derivadas de ordem superior na função de escoamento implica que apropriadas condições de contorno da variável de dano devem ser especificadas. Neste trabalho, estas condições de contorno adicionais são dadas na forma proposta por Mühlhaus\& Aifantis ${ }^{7}$ para 0 caso de plasticidade:

$\mathrm{G}[\nabla \mathrm{D} \cdot \mathrm{n}] \mathrm{D}=0$ em $\Gamma_{\mathrm{D}}, \quad$ para os modelos A e B.

$\left\{\mathrm{G} \nabla \mathrm{D} \cdot \mathrm{n}-\mathrm{G}_{2} \nabla[\Delta \mathrm{D}] \cdot \mathrm{n}+\mathrm{G}_{2}[\Delta \mathrm{D} \underset{\mathrm{div}}{\mathrm{s}}(\mathrm{n})-\stackrel{\mathrm{s}}{\operatorname{div}}[\Delta \mathrm{Dn}]]\right\} \dot{\mathrm{D}}=0 \quad \mathrm{em} \Gamma_{\mathrm{D}}$

$\left[\mathrm{C}_{2} \Delta \mathrm{D}\right] \stackrel{\mathrm{n}}{\nabla} \dot{\mathrm{D}}=0 \mathrm{em} \Gamma_{\mathrm{D}}$

para modelo C.

onde $\Gamma_{\mathrm{D}}$ é 0 contorno da região com $\dot{\mathrm{D}}>0.0$ gradiente $\nabla$ foi dividido em gradiente de superfície $\stackrel{\mathrm{s}}{\nabla}$ e gradiente normal $\mathrm{n} \stackrel{\mathrm{n}}{\nabla}: \nabla \stackrel{\mathrm{s}}{\nabla} \mathrm{n} \stackrel{\mathrm{n}}{\mathrm{\nabla}}$.

\subsubsection{Localização como singularidade do tensor acústico}

Conforme apresentado no capítulo anterior para o caso de plasticidade, para determinar as condições em que as equações governantes admitem solução não homogênea, além da homogênea inicial, supõe-se a existência de soluções do tipo:

$\mathrm{u}=\mathrm{v}_{0} \mathrm{e}^{\mathrm{iqn} \cdot \mathrm{x}} \quad \mathrm{D}=\mathrm{D}_{0} \mathrm{e}^{\mathrm{iqn} \cdot \mathrm{x}}$

onde né 0 vetor normal à banda de cisalhamento e qé o número de onda, $\mathrm{v}_{0}$ e $\mathrm{D}_{0}$ são amplitudes.

Este estudo é apresentado em Benallal \& Tvergard ${ }^{49}$ para 0 modelo de plasticidade proposto por Miblhaus \& Aifantis ${ }^{7}$ modificado com a consideração de grandes deformações, e em Comi \& Driemier ${ }^{50}$ para este modelo de dano.

Substituindo-se 4.12 na condição de equilíbrio estático:

$$
\operatorname{div}\left(\sigma^{\circ}\right)=\operatorname{dv}\left(E(1-D)^{2} \nabla \dot{U}-2 E(1-D)_{\varepsilon} \mathrm{D}\right)=0
$$


e na condição de consistência:

$$
\mathrm{f}=\mathrm{E}_{\varepsilon}(1-\mathrm{D}) \cdot \nabla \dot{\mathrm{u}}-\left(\frac{1}{2} \varepsilon \cdot \mathrm{E} \varepsilon+\mathrm{b}\right)+\mathrm{G} \Delta \mathrm{D}-\mathrm{C}_{2} \Delta^{2} \dot{\mathrm{D}}=0
$$

(por simplificação, considera-se $\mathrm{b}_{1}=\mathrm{b}_{2}=0$ e $\mathrm{b}_{3}=\mathrm{b}$ ) obtém-se, respectivamente:

$\mathrm{q}^{2}(1-\mathrm{D})^{2} \mathrm{E}(\mathrm{n} \otimes \mathrm{n}) \mathrm{v}_{0}-2 \mathrm{iq}(1-\mathrm{D}) \mathrm{nE} \varepsilon \mathrm{D}_{0}=0$

$\operatorname{iq}(1-\mathrm{D}) \mathrm{E}_{\varepsilon} \mathrm{n} \cdot \mathrm{v}_{0}+\left(\mathrm{h}+\mathrm{G} \mathrm{q}^{2}+\mathrm{C}_{2} \mathrm{q}^{4}\right) \mathrm{D}_{0}=0$

onde $\mathrm{h}=\frac{1}{2} \varepsilon \cdot \mathrm{E}_{\varepsilon}+\mathrm{b}$ é, por definição, o parâmetro de encruamento do material; e $g(q)=\mathrm{Gq}^{2}+\mathrm{G}_{2} \mathrm{q}^{4}$ é uma função que surge devido ao caráter não local do modelo.

Elimina-se $\mathrm{D}_{0}$ substituindo-se $4.15 \mathrm{~b}$ em 4.15a. Para solução diferente da trivial do sistema $4.15\left(\mathrm{v}_{0} \neq 0\right)$ :

$\operatorname{det}[\mathrm{Q}(\mathrm{n} \otimes \mathrm{n})]=0$

onde $\mathrm{Q}(\mathrm{n} \otimes \mathrm{n})$ é chamado tensor acústico e depende dos parâmetros do material danificado:

$$
\mathrm{Q}=(1-\mathrm{D})^{2} \mathrm{E}-\frac{(1-\mathrm{D}) \mathrm{E} \varepsilon \otimes(1-\mathrm{D}) \mathrm{E} \varepsilon}{\mathrm{h}+\mathrm{g}(\mathrm{q})}
$$

No caso, a condição de localização 4.16 pode ser interpretada como singularidade do tensor acústico e é conhecida como condição fraca de localização ou condição de compatibilidadedeMaxudl [Rizzi ${ }^{4}$.

Se existem, portanto, soluções bifurcadas de formas 4.12 em uma dada direção $\mathrm{n}$, então o número de onda é fixo e dado por:

$$
h+g(q)=[E(n \otimes n)]^{-1} \cdot[(E \varepsilon \otimes E \varepsilon)(n \otimes n)]
$$

Com G e G tendendo a zero, tem-se a condição clássica de localização para um contínuo local. Se h é o valor crítico de hpara o modelo local, ou seja, para o lado direito da equação 4.18, então:

$$
\min [g(q)]=\min [h-h]
$$


é 0 valor que dissipa a menor quantidade de energia durante 0 encruamento que precede a ruptura.

(a)

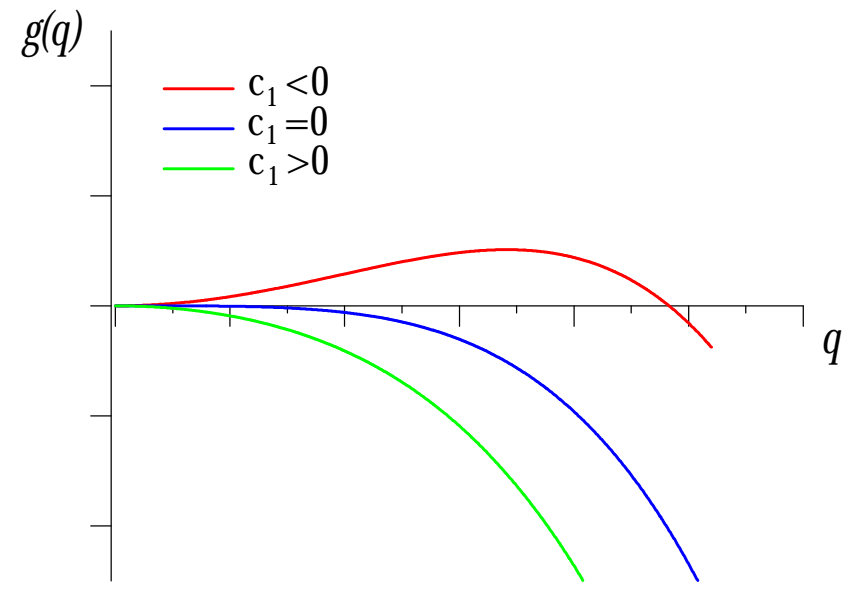

(b)

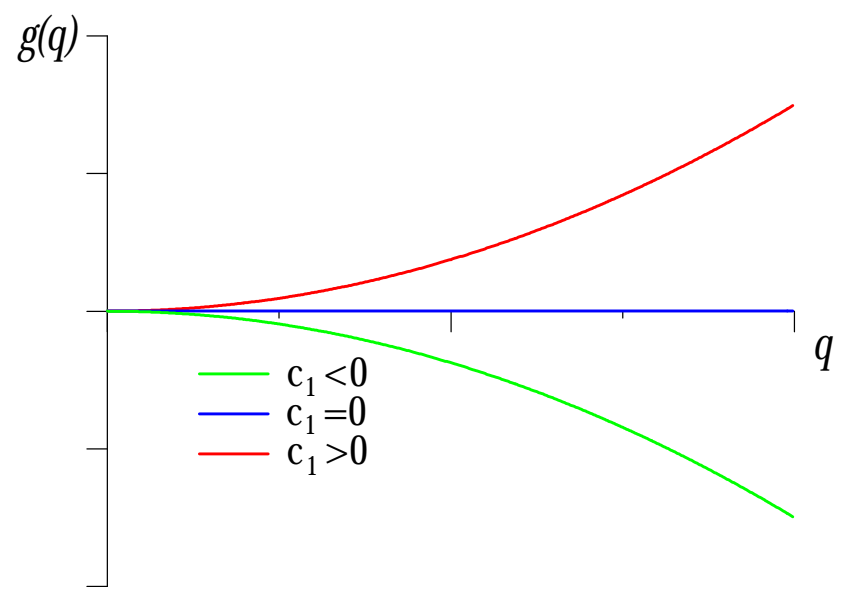

(c)

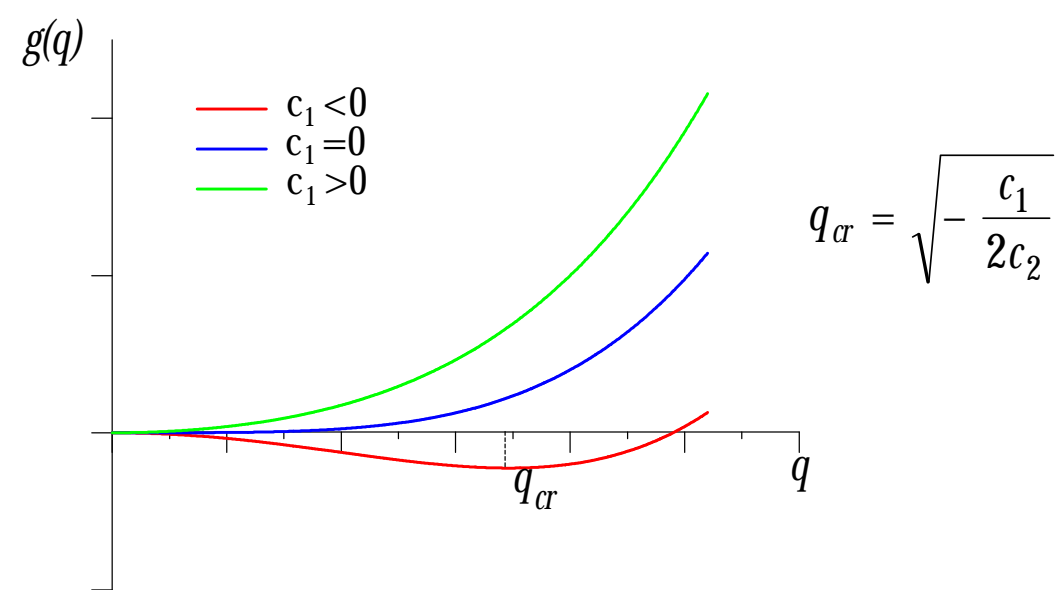

Figura 4.3. Gráfico g(q) x q para: (a) $\mathrm{C}_{2}<0$, (b) $\mathrm{C}_{2}=0$, e (c) $\mathrm{C}_{2}>0$. 
As Figuras 4.3a,b,c mostram a relação g(q) x q para o caso de, respectivamente, $\mathrm{C}_{2}<0, \mathrm{C}_{2}=0$ e $\mathrm{C}_{2}>0$.

Pela análise da Figura 4.3a, verifica-se que 0 mínimo ocorre para $\mathrm{q}_{\mathrm{c}} \rightarrow \infty$. Significa que não ocorrerá localização, ou seja, resposta homogênea em suttening

A Figura 4.3b mostra que para $\mathrm{G}_{1}>0$ e $\mathrm{c}_{2}=0$, tem-se 0 mínimo em $\mathrm{q}_{\mathrm{c}}=0$. Portanto, em uma análise estática, a bifurcação ocorre a partir do pico de tensão para modelos locais e não locais com gradiente de segunda ordem. Uma situação análoga ocorre para $\mathrm{G}_{1}>0$ e $\mathrm{G}_{2}>0$ (Figura 4.3c).

Para 0 caso de $\mathrm{G}<0$ e $\mathrm{G}_{2}>0$ (Figura 4.3c), $\mathrm{h}$ atinge 0 mínimo valor de $\mathrm{g}(\mathrm{q}$ ) correspondente a um número de onda crítico $\mathrm{q}_{\alpha}$, no qual a solução não homogênea começa a se desenvolver.

Convém ressaltar que através da análise estática, a largura da zona de localização não é identificável.

Para examinar as conseqüências do uso do modelo não local, convém analisar o caso unidimensional. A Figura 4.4 mostra a resposta homogênea uniaxial do modelo em termos de tensão- deformaçãa

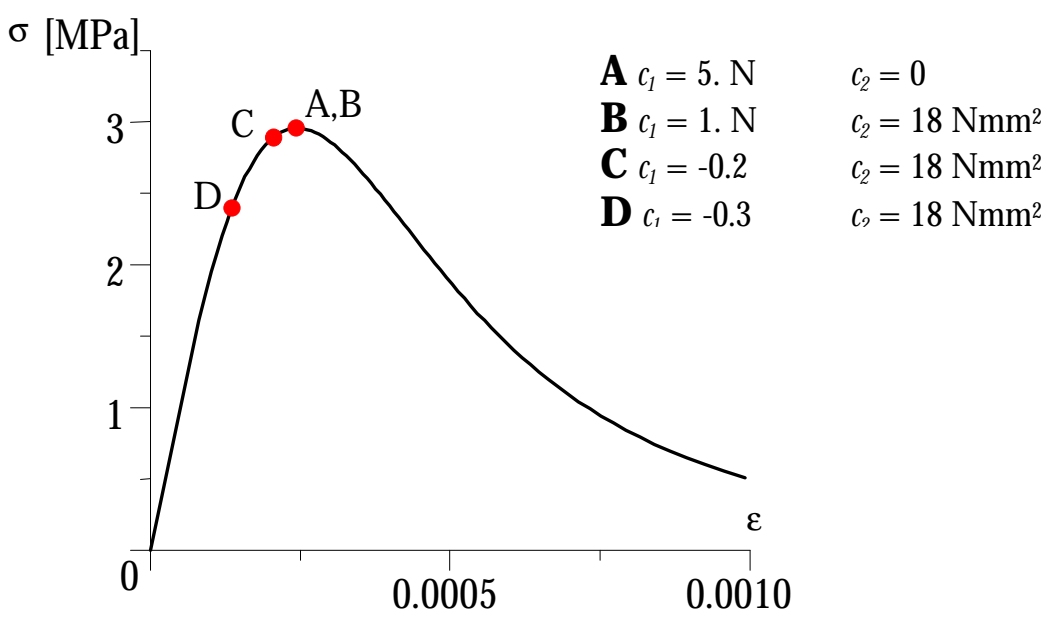

Figura 4.4. Resposta tensão - deformação uniaxial e pontos de localização para diferentes valores dos parâmetros de difusão. $\mathrm{E}=20000 \mathrm{MPa}, \mathrm{b}=.0018, \mathrm{k}=65 \mathrm{~Pa}$.

Portanto, a equação 4.18, para o unidimensional, pode ser rescrita na forma: 
$\frac{\mathrm{E}(1-\mathrm{D})^{2}}{\mathrm{~h}+\mathrm{G} \mathrm{q}^{2}+\mathrm{C}_{2} \mathrm{q}^{4}}\left[-\frac{3}{2} \mathrm{E}^{2}{ }^{2}+\mathrm{b}+\mathrm{G}_{\mathrm{q}} \mathrm{q}^{2}+\mathrm{C}_{2} \mathrm{q}^{4}\right]=0$

A condição crítica é obtida para:

$-\frac{3}{2} \mathrm{E}^{2}+\mathrm{b}+\mathrm{G} \mathrm{q}^{2}+\mathrm{C}_{2} \mathrm{q}^{4}=0$

Supondo-se um estado inicialmente homogêneo (com gradientes nulos de dano) e desfrutando-se da relação $\mathrm{f}=0$, a equação anterior pode ser equivalentemente expressa em termos da variável de dano:

$$
3 \frac{\mathrm{bD}+\mathrm{k}}{(1-\mathrm{D})}+\mathrm{G}^{2} \mathrm{q}^{2}+\mathrm{C}_{2} \mathrm{q}^{4}=0
$$

Deste modo, para valor de dano crítico do início da localização como sendo 0 menor valor que satisfaz a expressão 4.22, tem-se:

$$
D_{\mathrm{c}}=\min _{\mathrm{q}}[\mathrm{D}(\mathrm{q})] \quad \mathrm{D}(\mathrm{q})=3 \frac{\mathrm{b}-3 \mathrm{k}+\mathrm{G} \mathrm{q}^{2}+\mathrm{C}_{2} \mathrm{q}^{4}}{4 b+\mathrm{G} \mathrm{q}^{2}+\mathrm{C}_{2} \mathrm{q}^{4}}
$$

A Figura 4.5 mostra o valor do dano $\mathrm{D}(\mathrm{q})$ em função do número de onda qpara diferentes valores dos parâmetros de difusão.

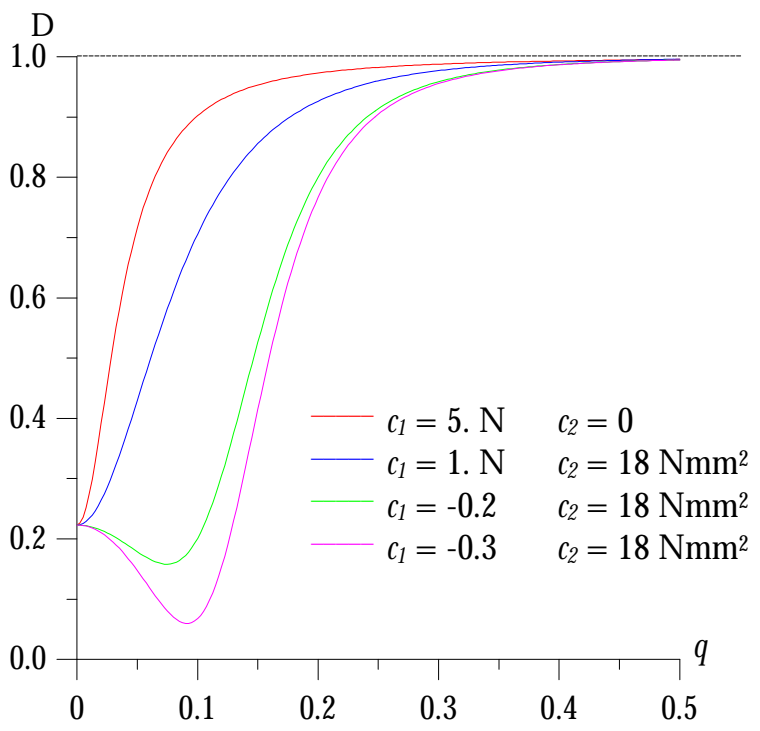

(a)

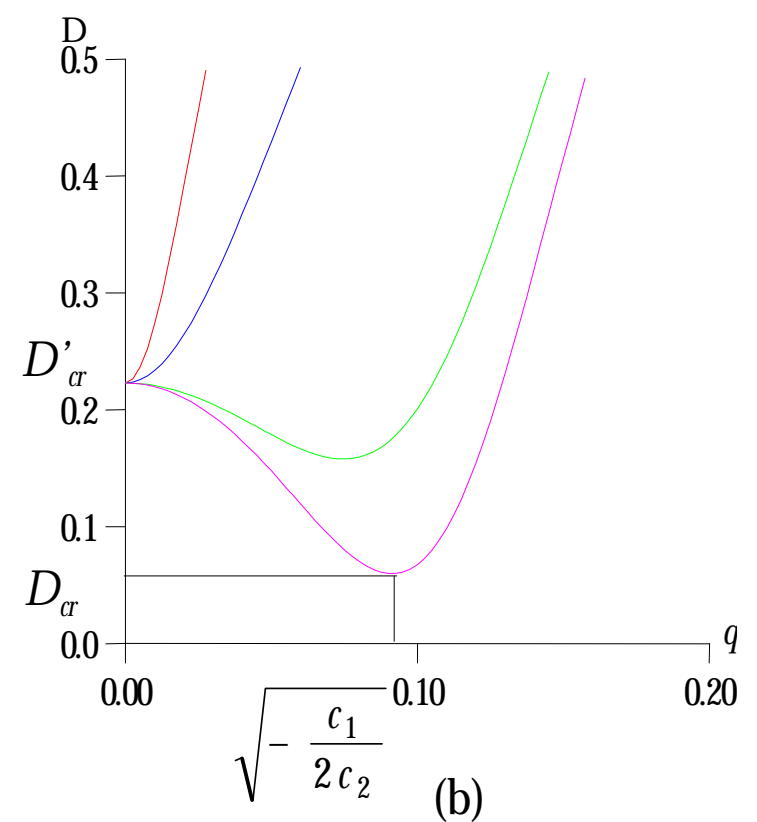

(b)

Figura 4.5.(a) Curva qx $\mathrm{D}_{\mathrm{\alpha}}$ e (b) Zomna curva(a) 
Portanto, teoricamente, o gradiente quarto prevê uma localização em um ponto diferente do pico da curva tensãox defomaçãa pois:

$$
\mathrm{D}_{\mathrm{p} \omega}=\left.\mathrm{D}\right|_{\frac{\partial \sigma}{\partial \varepsilon}=0}=\frac{\mathrm{b}-3 \mathrm{k}}{4 \mathrm{~b}} \equiv \mathrm{D}_{\mathrm{\alpha}}^{\prime}
$$

para os modelos local e não - local com gradiente de segunda ordem. Porém:

$$
\mathrm{D}_{\mathrm{C}}=\left.\mathrm{D}\right|_{\mathrm{q}=\sqrt{-\frac{\mathrm{c}_{1}}{2 \mathrm{c}_{2}}}}=\frac{\mathrm{b}-3 \mathrm{k}-\frac{\mathrm{q}^{2}}{2 \mathrm{c}_{2}}}{4 \mathrm{~b}-\frac{\mathrm{q}^{2}}{2 \mathrm{c}_{2}}}
$$

para modelo não - local com gradiente de quarta ordem. A Figura 4.4 apresenta os pontos de dano crítico, considerando-se os diferentes valores dos parâmetros de difusão utilizados nesta análise.

O u seja, como para os modelos com plasticidade, o modelo de dano com gradientes de segunda e quarta ordem prevê localização antes do pico de tensão, ou em hardkning para sinais particulares dos parâmetros de difusão.

\subsubsection{Propagação de onda}

As características de cada modelo proposto podem ser analisadas de maneira mais ampla no contexto dinâmico. Considera-se, por simplicidade, a propagação de uma onda unidimensional, conforme ilustrado no capítulo anterior para o caso de plasticidade.

Partindo-se de um estado homogêneo, com a propagação através de uma barra com dano de uma onda harmônica de freqüência $\omega$, número de onda q os campos de velocidade e de evolução do dano são expressos na forma:

$$
\dot{u}=v_{0} e^{i(q x-\omega t)} \quad \dot{D}=D_{0} e^{i(q x-\omega t)}
$$

A través da substituição direta nas seguintes equações de movimento:

$$
\dot{\sigma}_{, \mathrm{x}}=\rho \mathrm{u} \quad \dot{\sigma}=(1-\mathrm{D}) \mathrm{E}\left[(1-\mathrm{D}) \mathrm{u}_{, \mathrm{x}}-2 \dot{\mathrm{D}} \varepsilon\right] \quad \mathrm{f}=0
$$


segue que a velocidade de fase $\mathrm{C}_{\mathrm{f}} \equiv \omega / \mathrm{q}=\omega /(2 \pi / \lambda)$ depende do número de onda q. No caso, um meio dispersivo onde a velocidade de fase varia e as ondas se dispesam

Assumindo, por simplificação, na função de escoamento, $\mathrm{b}_{1}=\mathrm{b}_{2}=0$ e $\mathrm{b}_{3}=\mathrm{b}, \mathrm{a}$ velocidade de fase para os modelos A e B é:

$C_{f}=\frac{\omega}{q}=(1-D) c_{e} \sqrt{\frac{(1-a D)\left(b-\frac{3}{2} E \varepsilon^{2}\right)+a\left[(1-D) \frac{1}{2} E \varepsilon^{2}-b D-k\right]+G q^{2}}{(1-a D)\left(b+\frac{1}{2} E \varepsilon^{2}\right)+a\left[(1-D) \frac{1}{2} E \varepsilon^{2}-b D-k\right]+G q^{2}}}$

e para o modelo C é:

$c_{f}=\frac{\omega}{q}=c_{e}(1-D) \sqrt{\frac{b-\frac{3}{2} E \varepsilon^{2}+G q^{2}+c_{2} q^{4}}{b+\frac{1}{2} E \varepsilon^{2}+G q^{2}+c_{2} q^{4}}}$

onde $C_{\mathrm{e}} \equiv \sqrt{\mathrm{E} / \rho}$ é a velocidade de propagação elástica na barra.

Para o modelo local, a velocidade de propagação vale:

$C_{f}=\frac{\omega}{q}=(1-D) c_{\mathrm{e}} \sqrt{\frac{b-\frac{3}{2} E \varepsilon^{2}}{b+\frac{1}{2} E \varepsilon^{2}}}$

e não depende de qou $\omega$.

As velocidades de fase 4.28-4.29 são reais se o número de onda é tal que os numeradores destas equações são positivos. D este modo, tem-se que apenas ondas com comprimento $\lambda \leq \lambda_{\mathrm{c}}$ propagam-se, onde: 
$\lambda_{\mathrm{C}}=\frac{2 \pi}{\mathrm{q}_{\mathrm{C}}} \equiv 2 \pi \sqrt{\frac{2 \mathrm{c}_{1}}{(1-\mathrm{aD})\left(3 \mathrm{E} \varepsilon^{2}-2 \mathrm{~b}\right)+\mathrm{a}\left[2 \mathrm{bD}+2 \mathrm{k}-(1-\mathrm{D}) \mathrm{E} \varepsilon^{2}\right]}}$

(modelos A, B)

ou

$\lambda_{\mathrm{C}}=\frac{2 \pi}{\mathrm{q}_{\mathrm{C}}} \equiv 2 \pi \sqrt{\frac{2 \mathrm{c}_{2}}{-\mathrm{c}_{1}+\sqrt{\mathrm{C}_{1}^{2}+4 \mathrm{c}_{2}\left(\frac{3}{2} \mathrm{E}^{2}-\mathrm{b}\right)}}} \quad$ (modelo C)

Devido ao comportamento dissipativo do material altas freqüências são desprezadas, e obtém-se uma onda de localização harmônica estacionária de comprimento de onda $\lambda=\lambda_{\text {c; }}$ este comprimento representa 0 comprimento interno de localização.

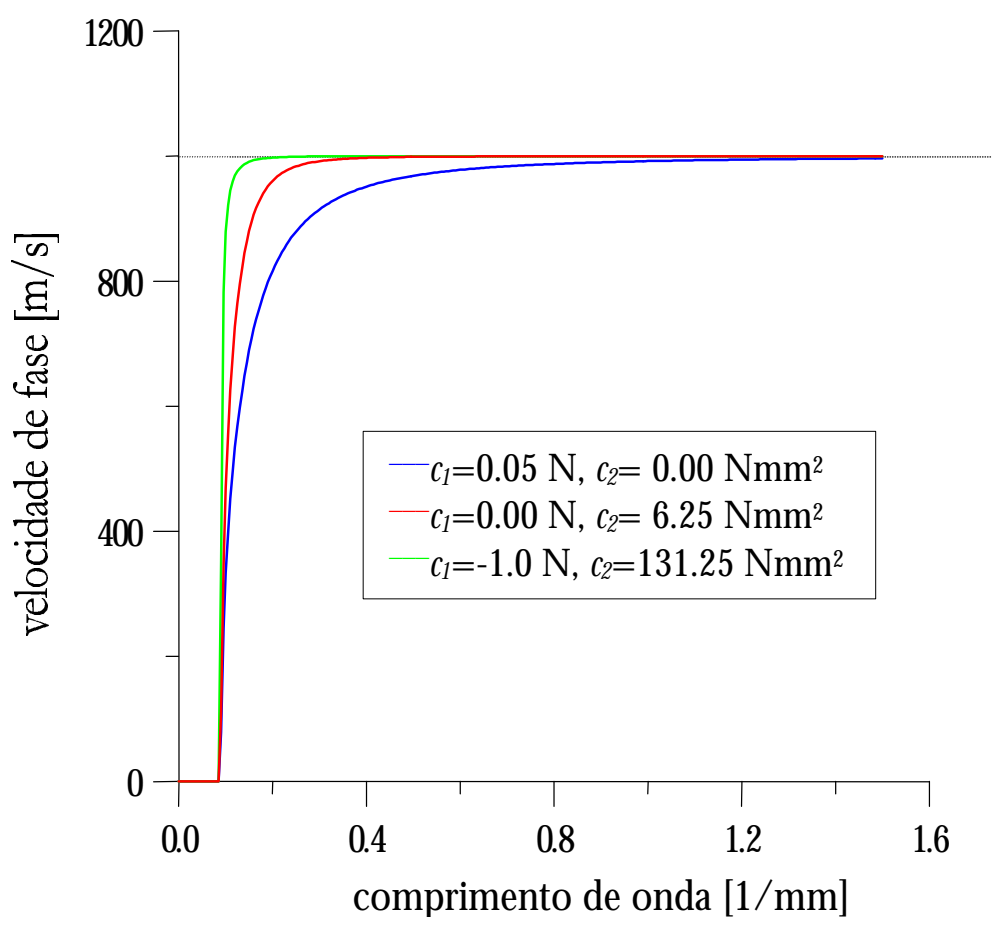

Figura 4.6. Velocidade de fase para modelos de dano A, B e C.

A evolução da velocidade de fase com o número de onda é mostrado na Figura 4.6, para $\mathrm{D}=0$ e parâmetros do material: $\mathrm{E}=20000 \mathrm{MPa}, \mathrm{c}_{\mathrm{e}}=1000 \mathrm{~m} / \mathrm{s}$, $\mathrm{b}=\mathrm{k}=0.0002 \mathrm{MPa}$ e $\mathrm{G}, \mathrm{G}$ que forneçam o mesmo número de onda crítico $\mathrm{q}_{\mathrm{c}}=0.089 \mathrm{~mm}^{-1}$. Apesar do comportamento qualitativo semelhante, i.e. a velocidade de fase cresce de zero à velocidade de propagação elástica, a presença 
do gradiente de quarta ordem induz à um crescimento mais rápido com o número de onda.

Considerem-se estados homogêneos iniciais diferentes, caracterizados por valores fixos de dano e deformação pertencentes à função de escoamento, i. e.:

$$
\frac{1}{2}(1-\mathrm{D}) \mathrm{E} \varepsilon^{2}=\mathrm{bD}+\mathrm{k}
$$

D as equações 4.31 e 4.33, o comprimento característico apresenta a seguinte forma para os modelos A e B:

$$
\lambda_{C}=2 \pi \sqrt{\frac{G(1-D)}{(1-a D)(3 k-b+4 b D)}}
$$

D eve-se ressaltar que, diferentemente do caso da plasticidade ilustrado no capítulo anterior, se $\mathrm{a}=0$ a largura da zona de localização diminui com 0 aumento do dano, iniciando com o valor $2 \pi \sqrt{\frac{\mathrm{G}}{3 \mathrm{k}-\mathrm{b}}}$ e tendendo a zero quando o dano tende ao valor crítico um (Figura 4.7, curva 3). Como conseqüência, o efeito de regularização do termo gradiente tende a desaparecer. Este comportamento, que se por um lado é fisicamente admissível, uma vez que permite a simulação da transição de uma zona altamente danificada para uma macrofissura, por outro lado, requer um refinamento de malha adaptativo ou a transição de uma aproximação contínua para uma discreta na qual descontinuidades de deslocamento são consideradas.

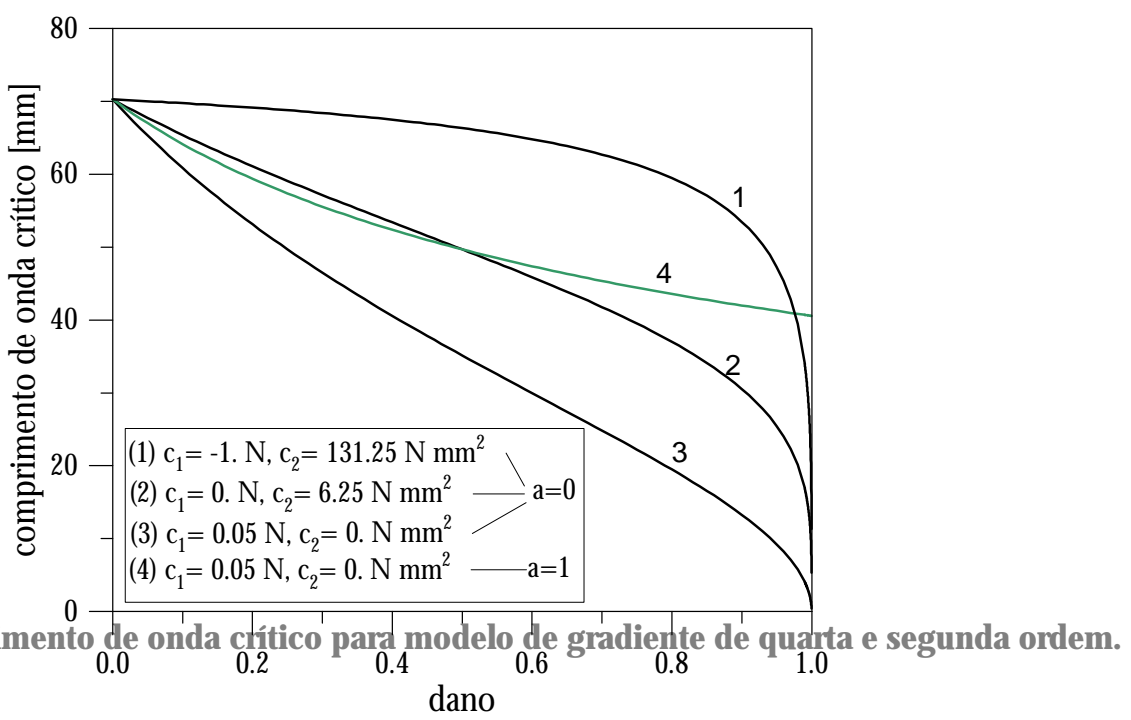


Se a $=1$, o comprimento característico também diminui com o dano, mas tende a um valor positivo não nulo $2 \pi \sqrt{\frac{\mathrm{G}}{3 \mathrm{k}+3 \mathrm{~b}}}$ se $\mathrm{D}$ tende a um. Neste caso, a zona de localização não se torna uma linha (Figura 4.7, curva 4). Recupera-se, portanto, a objetividade de resposta em uma análise numérica com uma largura finita de banda.

Das equações 4.32 e 4.33, tem-se para o modelo C:

$$
\lambda_{c}=2 \pi \sqrt{\frac{2 \mathrm{C}_{2} \sqrt{1-D}}{-\mathrm{G}_{1} \sqrt{1-\mathrm{D}}+\sqrt{\mathrm{G}^{2}(1-\mathrm{D})+4 \mathrm{G}_{2}(3 k-b+4 \mathrm{bD})}}}
$$

Novamente a largura da banda diminui para aumento do dano, tendendo a zero quando o dano tende ao valor crítico um, mas vai a zero mais lentamente que no caso $\mathrm{C}_{2}=0$ (compare curvas 1 e 2 com curva 3 na Figura 4.7). 


\section{IMPLEMENTAÇÃO DO MODELO DE DANO}

\subsection{Introdução}

A análise de sólidos ou de estruturas em regime anelástico sujeitas a uma dada história de carregamento requer uma dupla discretização, no espaço e no tempo, para obter resultados numéricos.

A discretização no espaço tem como objetivo definir aproximações para a solução do problema de análise estrutural expresso por equações diferenciais não - lineares e inequações. $\mathrm{O}$ método dos elementos finitos é uma importante ferramenta usada com este objetivo, modelando campos desconhecidos através de convenientes interpolações sobre o domínio.

A discretização no tempo visa reduzir as equações diferenciais mencionadas acima em uma seqüência de problemas algébricos não-lineares aproximados (incrementos ou passos finitos).

Como já visto, em modelos com gradiente, a relação constitutiva envolve as variáveis de estado e suas derivadas espaciais. Vários autores propõem uma maneira para incorporar estas características em uma estratégia numérica apropriada ao contexto do método dos elementos finitos, entre eles cita-se Comi \& Peeeg ${ }^{51}$ de Barst \& Müblhaus $^{29}$, Sluys ${ }^{8}$.

Neste capítulo, discretiza-se o modelo de dano com gradiente no espaço, com uma formulação em elementos finitos, proposta inicialmente por Conadi ${ }^{52,53}$, reestudada 
por Comi \& al. ${ }^{19}$ e utilizada no estudo de modelos de plasticidade com gradiente por Comi \& Peeego ${ }^{51,54}$.

Existem outras estratégias em elementos finitos para implementação de modelos com gradiente (que não serão utilizada neste trabalho), cita-se, por exemplo, de Barst \& Mïhlhaus ${ }^{29}$, Slyus ${ }^{8}$.

Observa-se que na etapa de implementação computacional, adicionaram-se subrotinas relativas ao modelo de dano com gradiente no programa de Elementos Finitos sugerido por Zienkievicz \& Taylor ${ }^{55,56}$.

\subsection{Problema em passo finito}

Considere-se a evolução de um corpo de volume $\Omega$ e contorno $\Gamma$, sujeito a forças de volume $\mathrm{R}(\mathrm{t})$ forças de superfície $\mathrm{r}(\mathrm{t})$ em $\Gamma_{\mathrm{f}}$, descrita pelo modelo de dano do capítulo anterior e equações de equilíbrio dinâmico e compatibilidade linear.

Supõe-se $t_{0}, t_{1}, \ldots, t_{n}, t_{n+1}=t_{n}+\Delta t, \ldots$ instantes de tempo ao longo do intervalo onde a resposta dinâmica da estrutura está sendo observada, sendo que em $t=t_{n}$ todos valores são conhecidos, e a solução deve ser computada em $t_{n+1}$ para dados incrementos de carga DR e Dr.

Utilizam-se as aproximações:

$$
\begin{aligned}
& \operatorname{Du}=\operatorname{Dt} \dot{u}_{n+\gamma}=\operatorname{Dt}\left[(1-\gamma) u_{n}+\gamma u_{n+1}\right] \\
& \operatorname{Du}=\operatorname{Dt} u_{n+\gamma}=\operatorname{Dt}\left[(1-\gamma) u_{n}+\gamma u_{n+1}\right]
\end{aligned}
$$

com parâmetro $\gamma \in] 0$ 1] para velocidades e acelerações (subscritos $n, n+1$ e $n+\gamma$ representam valores em $t_{n}, t_{n+1}$ e $t_{n+\gamma}$ respectivamente, $D \cdot$ representa 0 incremento da variável • sobre Dt).

0 problema dinâmico de passo finito é obtido impondo equilíbrio dinâmico e compatibilidade geométrica no final do passo: resolvendo a equação 5.1 e integrando a lei constitutiva de acordo com um esquema implícito:

$$
\mathrm{L}^{\mathrm{T}} \sigma_{\mathrm{n}+1}+\mathrm{R}_{\mathrm{n}+1}^{*}=\rho^{*} \mathrm{u}_{\mathrm{n}+1} \text { em } \Omega ; \quad \quad \mathrm{m}_{\mathrm{n}+1}=\mathrm{r}_{\mathrm{n}+1} \text { em } \Gamma_{\mathrm{f}}
$$


$\mathrm{Cu}_{\mathrm{n}+1}=\varepsilon_{\mathrm{n}+1}$ em $\Omega ; \quad \mathrm{u}_{\mathrm{n}+1}=\mathrm{U}_{\mathrm{n}+1}$ em $\Gamma_{\mathrm{u}}$

$\sigma_{\mathrm{n}+1}=\left(1-\mathrm{D}_{\mathrm{n}+1}\right)^{2} \mathrm{E}_{\varepsilon_{\mathrm{n}+1}}$ em $\Omega$

$\mathrm{f}_{\mathrm{n}+1} \leq 0 \quad$ em $\Omega$

$\mathrm{DD} \geq 0 \quad \mathrm{f}_{\mathrm{n}+1} \mathrm{DD}=0 \quad$ em $\Omega$

$\mathrm{G}[\nabla \mathrm{D} \cdot \mathrm{n}] \mathrm{D}=0$ emG,$\quad$ para os modelos $\mathrm{A}$ e B.

$\left\{\mathrm{G}_{\mathrm{q}} \Delta \mathrm{D}_{\mathrm{n}+1} \mathrm{n}-\mathrm{C}_{2} \nabla\left[\Delta \mathrm{D}_{\mathrm{n}+1}\right] \cdot \mathrm{n}+\mathrm{C}_{2}\left[\Delta \mathrm{D}_{\mathrm{n}+1} \mathrm{div}(\mathrm{n})-\mathrm{div}\left[\Delta \mathrm{D}_{\mathrm{n}+1} \mathrm{n}\right]\right]\right\} \mathrm{DD}_{\mathrm{n}+1}=0$ $e m G_{D}$

$\left[\mathrm{C}_{2} \Delta \mathrm{D}_{\mathrm{n}+1}\right] \stackrel{\mathrm{n}}{\nabla} \mathrm{DD} \mathrm{D}_{\mathrm{n}+1}=0 \mathrm{emG}_{\mathrm{D}}$

para modelo C.

Nas relações acima, C é o operador diferencial de compatibilidade linear; mé a normal à superfície $\Gamma$; U é o vetor de deslocamentos impostos. Convém lembrar que os modelos A, B, C referem-se àqueles apresentados no Capítulo 4, a partir da equação 4.9, para valores particulares dos parâmetros de difusão G, ce e da constante a.

Ainda, $\rho{ }^{*}$ e $\mathrm{R}_{\mathrm{n}+1}^{*}$ são definidos como:

$$
\rho^{*} \equiv \frac{1}{\gamma^{2} \Delta \mathrm{t}^{2}} \rho ; \quad \mathrm{R}_{\mathrm{n}+1}^{*} \equiv \mathrm{R}_{\mathrm{n}+1}+\rho\left[\frac{1-\gamma}{\gamma} \mathrm{u}_{\mathrm{n}}+\frac{1}{\gamma^{2} \Delta \mathrm{t}} \mathrm{u}_{\mathrm{n}}+\frac{1}{\gamma^{2} \Delta \mathrm{t}^{2}} \mathrm{u}_{\mathrm{n}}\right]
$$

0 algoritmo de integração acima apresenta os parâmetros de Newmark $\gamma^{*},{ }^{*}{ }^{*}$ de modo que: $\gamma^{*}=2 \beta^{*} ; \rho$ é a densidade de massa. Para $\gamma=1 / 2$ (i.e. $\beta^{*}=1 / 4$, $\gamma^{*}=1 / 2$ ) obtém-se 0 mítodbdeacderacão média e faz parte do programa de elementos finitos de Zienkienicz \& Taylar ${ }^{55,56}$. 


\subsection{Formulação variacional}

Utiliza-se uma formulação variacional para o problema em passo finito. Neste trabalho, elaboram-se dois funcionais independentes para as fases de previsão e correção do problema não linear, conforme sugere Comi ${ }^{10}$ para implementação numérica de modelos com gradientes em variáveis generalizadas. Balbo ${ }^{18}$, sob uma análise matemática, também propõe a resolução do problema procurando-se condições ótimas de dois funcionais distintos.

Uma vez apresentados os funcionais, cada variável do funcional será discretizada independentemente, conforme 0 método dos elementos finitos em variáveis generalizadas. O Anexo B apresenta uma breve discussão sobre as variáveis generalizadas. As equações resultantes das condições ótimas dos funcionais discretizados são implementadas no programa em elementos finitos de Zienkievicz \& Taylar ${ }^{55,56}$.

\subsubsection{Fase de previsão}

A formulação variacional da fase de previsão na forma de um princípio tipo $\mathrm{Hu}$ Washizu pode ser estabelecida, como em $\mathrm{Comi}^{10}$. Ou seja, a fase de previsão é equivalente ao seguinte problema de ponto - sela:

$$
\begin{aligned}
& \min _{\mathrm{u}, \varepsilon} \max _{\sigma}\left\{\mathbf{L}_{\mathbf{p}}(\mathrm{u}, \varepsilon, \sigma)\right\} \\
& \mathbf{L}_{\mathbf{p}} \equiv \int_{\Omega} \frac{1}{2} \rho^{*} \mathrm{u}^{\mathrm{T}} \mathrm{ud} \Omega+\int_{\Omega} \frac{1}{2}\left(1-\mathrm{D}_{\mathrm{n}}\right)^{2} \varepsilon^{\mathrm{T}} \mathrm{E} \varepsilon \mathrm{d} \Omega+\int_{\Omega} \sigma^{\mathrm{T}}(\mathrm{Cu}-\varepsilon) \mathrm{d} \Omega \\
& -\int_{\Omega} \mathrm{R}^{\mathrm{iT}} \mathrm{ud} \Omega-\int_{\Gamma \mathrm{r}} \mathrm{r}^{\mathrm{T} \mathrm{T}} \mathrm{ud} \boldsymbol{}
\end{aligned}
$$

A fasedepreisãocoincide com um problema dinâmico (integrado no tempo para obter um problema equivalente quase - estático) para um corpo elástico com módulo de elasticidade $E\left(1-D_{n}\right)^{2}$. Stdarski \& Bdytschko ${ }^{57}$ analisam este tipo de problema baseando-se no clássico princípio misto da elasticidade Hu-Washizu.

\subsubsection{Fase de correção}

Para os valores de $\varepsilon$ obtidos na fase de previsão $\left(\varepsilon \equiv \varepsilon^{\mathrm{i}}\right)$ pode-se escrever dois variacionais para a fase de correção que diferem entre si somente da forma de discretização adotada. A solução dos seguintes problemas de minimização: 


$$
\begin{aligned}
& \min _{\Delta \mathrm{D}}\left\{\mathbf{L}_{\mathbf{c}}\right\} \quad \Delta \mathrm{D} \in \mathbf{K} ; \quad \mathbf{K} \equiv\left\{\Delta \mathrm{D} \in \mathrm{C}^{1} \mid \Delta \mathrm{D} \geq 0 \text { em } \Omega\right\} \\
& \mathbf{L}_{\mathbf{c}}(\Delta \mathrm{D}) \equiv \int_{\Omega}^{\lceil}\left[\frac{1}{2}(1-\mathrm{D})^{2}{ }_{\varepsilon}^{\mathrm{T}} \mathrm{E} \varepsilon-2\left(\mathrm{~b}_{1} \mathrm{P}^{\mathrm{T}} \varepsilon-\mathrm{k}\right) \mathrm{D}-\left(\mathrm{b}_{1}\left(\mathrm{~b}_{2}-1\right) \mathrm{P}^{\mathrm{T}} \varepsilon-\mathrm{b}_{3}\right) \mathrm{D}^{2}+\frac{2}{3} \mathrm{~b}_{1} \mathrm{~b}_{2} \mathrm{P}^{\mathrm{T}}{ }_{\varepsilon} \mathrm{D}^{3}\right] \mathrm{d} \Omega+ \\
& \left.\mathrm{a} \int_{\Omega}^{\lceil}\left[\frac{1}{2} \mathrm{D}^{2}\left(1-\frac{2}{3} \mathrm{D}\right)\right)^{\mathrm{T}} \mathrm{E} \varepsilon+\left(\mathrm{b}_{1} \mathrm{P}^{\mathrm{T}} \varepsilon-\mathrm{k}\right) \mathrm{D}^{2}+\frac{2}{3}\left(\mathrm{~b}_{1}\left(\mathrm{~b}_{2}-1\right) \mathrm{P}^{\mathrm{T}} \varepsilon-\mathrm{b}_{3}\right) \mathrm{D}^{3}-\frac{1}{2} \mathrm{~b}_{1} \mathrm{~b}_{2} \mathrm{P}^{\mathrm{T}}{ }_{\varepsilon} \mathrm{D}^{4}\right] \mathrm{d} \Omega+ \\
& +\int_{\Omega} \mathrm{G}(\nabla \mathrm{D})^{\mathrm{T}} \nabla \mathrm{Dd} \Omega
\end{aligned}
$$

para os modelos A e B apresentados no Capítulo 4, e

$$
\begin{aligned}
& \min _{\Delta \mathrm{D}}\left\{\mathbf{L}_{\mathbf{c}}\right\} \quad \Delta \mathrm{D} \in \mathbf{K} ; \quad \mathbf{K} \equiv\left\{\Delta \mathrm{D} \in \mathrm{C}^{1} \mid \Delta \mathrm{D} \geq 0 \text { em } \Omega\right\} \\
& \boldsymbol{L}_{\boldsymbol{c}}(\mathrm{DD}) \equiv \int_{\Omega}\left[\frac{1}{2}(1-\mathrm{D})^{2}{ }_{\varepsilon}^{\mathrm{T}} \mathrm{E} \varepsilon-2\left(\mathrm{~b}_{1} \mathrm{P}^{\mathrm{T}} \varepsilon-\mathrm{k}\right) \mathrm{D}-\left(\mathrm{b}_{1}\left(\mathrm{~b}_{2}-1\right) \mathrm{P}^{\mathrm{T}} \varepsilon-\mathrm{b}_{3}\right) \mathrm{D}^{2}\right] \mathrm{d} \Omega \\
& +\int_{\Omega} \frac{2}{3} \mathrm{~b}_{1} \mathrm{~b}_{2} \mathrm{P}^{\mathrm{T}} \mathrm{D}^{3} \mathrm{~d} \Omega+\int_{\Omega} \mathrm{G}(\nabla \mathrm{D})^{\mathrm{T}} \nabla \mathrm{Dd} \Omega+\int_{\Omega} \mathrm{c}_{2}\left(\nabla^{2} \mathrm{D}\right)^{\mathrm{T}} \nabla^{2} \mathrm{Dd} \Omega
\end{aligned}
$$

para o modelo C apresentado no Capítulo 4, são também solução da fase de correção. Pé o vetor de projeção isótropa ( $\mathrm{P}^{\mathrm{T}} \equiv\left\{\begin{array}{llllll}1 & 1 & 1 & 0 & 0 & 0\end{array}\right)$ e $\mathbf{K}$ é o cone convexo de funções não - negativas e contínuas em suas primeiras derivadas. Para soluções fisicamente admissíveis, a solução deve ser restrita a $\mathrm{D}=\mathrm{D}_{\mathrm{n}}+\Delta \mathrm{D} \leq 1$.

Uma formulação mista alternativa pode ser obtida para o modelo C pela introdução do gradiente do dano $Q$ como uma variável independente, adicionando ao funcional a restrição $Q=\nabla D$, ponderada por um mutiplicador de lagrange $\mu$.

Esta formulação requer continuidade $\mathrm{C}^{0}$ para o campo de dano e é mais conveniente para a formulação em elementos finitos. 


$$
\begin{aligned}
& \min _{\Delta \mathrm{D}} \max _{\mu}\left\{\mathbf{L}_{\mathbf{c}}^{*}\right\} \quad \Delta \mathrm{D} \in \mathbf{K}^{*} ; ; \quad \mathbf{K}^{*} \equiv\left\{\Delta \mathrm{D} \in \mathrm{C}^{0} \mid \Delta \mathrm{D} \geq 0 \text { em } \Omega\right\} \\
& \mathbf{L}_{\mathbf{c}}{ }^{*}(\Delta \mathrm{D}, \Theta ; \mu) \equiv \int_{\Omega}^{[}\left[\frac{1}{2}(1-\mathrm{D})^{2}{ }_{\varepsilon}^{\mathrm{T}} \mathrm{E}_{\varepsilon}-2\left(\mathrm{~b}_{1} \mathrm{P}^{\mathrm{T}} \varepsilon-\mathrm{k}\right) \mathrm{D}-\left(\mathrm{b}_{1}\left(\mathrm{~b}_{2}-1\right) \mathrm{P}^{\mathrm{T}}-\mathrm{b}_{3}\right) \mathrm{D}^{2}\right] \mathrm{d} \Omega \\
& +\int_{\Omega} \frac{2}{3} \mathrm{~b}_{1} \mathrm{~b}_{2} \mathrm{P}^{\mathrm{T}}{ }_{\varepsilon} \mathrm{D}^{3} \mathrm{~d} \Omega+\int_{\Omega} \mathrm{G} \Theta^{2} \mathrm{~d} \Omega+\int_{\Omega} \mathrm{G}_{2}(\nabla \Theta)^{\mathrm{T}} \nabla \Theta \mathrm{d} \Omega+\int_{\Omega} 2 \mu(\nabla \mathrm{D}-\Theta) \mathrm{d} \Omega
\end{aligned}
$$

Uma importante característica da formulação é que o incremento de dano no problema de mínimo 5.12 e de ponto - sela 5.13 possui a restrição somente de não ser negativo. Além disso, pode-se provar que as condições de contorno (equações 5.7 e 5.8) são satisfeitas implicitamente para os funcionais acima, conforme demonstram Comi \& Peeeg ${ }^{15}$ para 0 caso de modelo elastoplástico com gradiente de segunda ordem, e Com ${ }^{10}$ e Comi \& Driemie ${ }^{11}$ para modelo elástico com dano com gradiente segundo e gradientes segundo e quarto, respectivamente.

\subsection{Processo iterativo de solução}

O problema não linear do valor de contorno no passo finito deve ser resolvido através de um processo iterativo. No esquema adaptado, cada iteração consiste em duas fases: uma fase linear de previsão e uma fase não linear de correção (a Figura 5.1 mostra, de maneira esquemática, a seqüência implementada, para as duas fases), e um teste de convergência:

- Fase de previsão - Na iteração i+1 para um valor de dano fixo, igual ao valor encontrado no final do passo anterior $\mathrm{D}=\mathrm{D}_{\mathrm{n}}$, e com forças externas iguais às forças residuais da iteração anterior $i$, compute 0 vetor estimado dos deslocamentos nodais $\mathrm{u}^{\mathrm{i}+1}$ das equações lineares de equilíbrio e compatibilidade e lei de Hooke.

口 Fasedecomesãa- com os valores das deformações da fase de previsão $\varepsilon^{i+1}=\mathrm{Cu}^{\mathrm{i}+1}$, compute 0 incremento de dano usando as condições de carregamento descarregamento e as condições de contorno com $D_{n+1}=D_{n}+D^{i+1}$ e $\varepsilon_{\mathrm{n}+1}=\varepsilon^{\mathrm{i}+1}$. Com 0 valor do dano calculado, atualize as tensões.

口 Testedeconvegênia - Compute 0 vetor de forças residuais $\mathrm{R}^{\mathrm{i}+1}$ e $\mathrm{r}^{\mathrm{i}+1}$ com 0 valor corrente das tensões e cheque a convergência comparando uma norma dos resíduos com um valor fixo da tolerância. Se não passou no teste, faça nova iteração. 


\section{SOLUÇÃO ITERATIVA: ESQUEMA \\ 口previsão elástica \\ $\square$ correção não linear do dano}
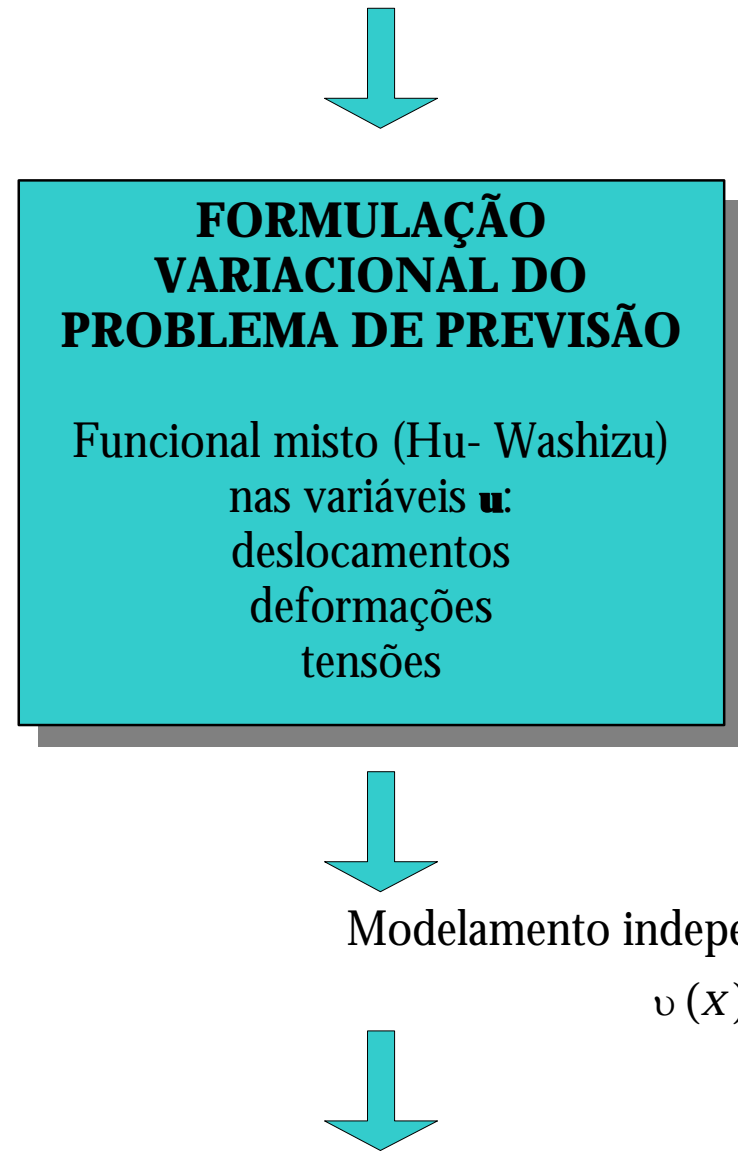

\section{Propriedades do funcional discretizado}

\section{Condições de estacionariedad ค}

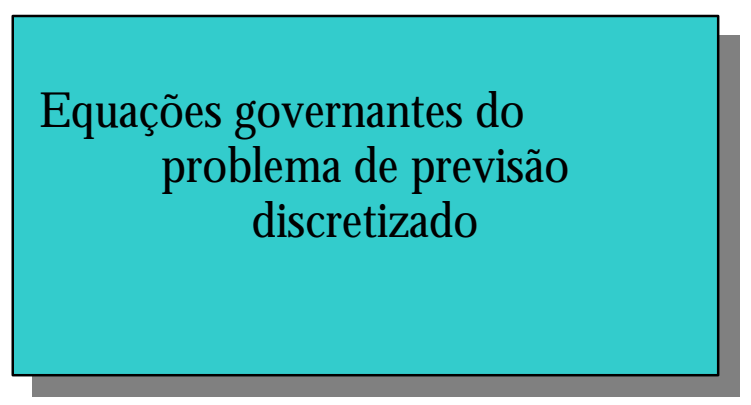

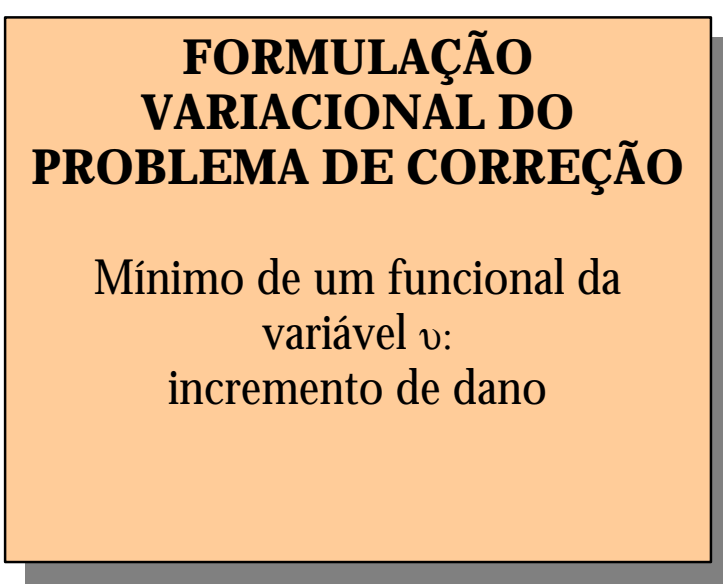

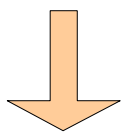

os campos:

$v(\mathrm{x})=\mathrm{N}_{\mathrm{v}} \bar{v}$

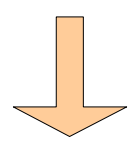




\subsection{Implementação numérica}

A Tabela 5.1 descreve os elementos implementados, que serão utilizados no capítulo seguinte.

\begin{tabular}{|c|c|c|}
\hline ELEMENTO & DESCRIÇÄO & SÍMBOLO \\
\hline & Elemento de barra* & TRU0-2 \\
\hline & $\begin{array}{c}\text { Elemento de barra } \\
\text { Comi }^{10} \text {, Comi \& Drieriee } \\
{ }^{11}\end{array}$ & TRU-2 \\
\hline & $\begin{array}{l}\text { CST - elemento triangular a deformação } \\
\text { plana } \\
\text { Comi }^{10}, \text { Cài \& Driemeier }\end{array}$ & CST-3 \\
\hline & $\begin{array}{l}\text { Elemento de barra } \\
\text { Comi \& Driemie }{ }^{11}\end{array}$ & TRU-3 \\
\hline
\end{tabular}

Tabela 5.1. Elementos finitos implementados.

\subsubsection{Elemento TRU0-2}

Elemento TRU0-2 é um elemento de barra com interpolação constante para tensões, deformações e dano, e interpolação linear para deslocamentos. Sobre a implementação, não apresenta nenhum ponto a ser discutido, uma vez que se trata de um elemento simples e clássico em elementos finitos. Implementado com o modelo local de dano, este elemento é utilizado somente para mostrar a dependência da discretização nos resultados obtidos.

\subsubsection{Elemento TRU-2}

Elemento TRU-2 é um elemento de barra com interpolação constante para tensões e deformações, e interpolação linear para deslocamentos e variável de dano. 


\subsubsection{Discretização do funcional}

$\mathrm{N}_{\mathrm{u}}, \mathrm{N}_{\varepsilon}, \mathrm{N}_{\mathrm{D}}$ e $\mathrm{N}_{\sigma}$ são as matrizes globais de funções de interpolação definidas sobre todo o conjunto de elementos da estrutura. Cada campo (deslocamentos, deformações, tensões e incrementos de dano) será modelado separadamente em termos de parâmetros independentes (representados por barras sobre a letra que representa a variável); 0 índice hrepresenta campos discretizados:

$$
\begin{aligned}
& \mathrm{u}^{\mathrm{h}}(\mathrm{x})=\mathrm{N}_{\mathrm{u}}(\mathrm{x}) \mathrm{u}, \varepsilon^{\mathrm{h}}(\mathrm{x})=\mathrm{N}_{\varepsilon}(\mathrm{x}) \bar{\varepsilon} \\
& \Delta \mathrm{D}^{\mathrm{h}}(\mathrm{x})=\mathrm{N}_{\mathrm{D}}(\mathrm{x}) \Delta \overline{\mathrm{D}}, \sigma^{\mathrm{h}}(\mathrm{x})=\mathrm{N}_{\sigma}(\mathrm{x}) \bar{\sigma}
\end{aligned}
$$

\subsection{Fase de previsão}

0 funcional da fase de previsão, conforme equações 5.10, discretizado:

$$
\begin{aligned}
& \min _{\delta \bar{u}, \delta \bar{\varepsilon}} \max _{\delta \bar{\sigma}}\left\{\overline{\mathbf{L}}_{\mathbf{p}}\right\} \operatorname{com} \delta \mathrm{u}=0 \text { on } \Gamma_{\mathrm{u}} \\
& \mathbf{L}_{\mathbf{p}} \equiv \int_{\Omega} \frac{1}{2} \rho^{*} \delta \mathrm{u}^{\mathrm{T}} \mathrm{N}_{\mathrm{u}}^{\mathrm{T}} \mathrm{N}_{\mathrm{u}} \delta \mathrm{u} \mathrm{d} \Omega+\int_{\Omega} \frac{1}{2}\left(1-\mathrm{N}_{\mathrm{D}} \overline{\mathrm{D}}_{\mathrm{n}}\right)^{2} \delta \bar{\varepsilon} \mathrm{T}_{\mathrm{N}_{\varepsilon}^{\mathrm{T}}}^{\mathrm{T}} \mathrm{EN}_{\varepsilon} \delta \bar{\varepsilon} \mathrm{d} \Omega+ \\
& +\int_{\Omega} \delta \bar{\sigma}^{\mathrm{T}} \mathrm{N}_{\sigma}^{\mathrm{T}}\left(\mathrm{C} \mathrm{N}_{\mathrm{u}} \delta \mathrm{u}-\mathrm{N}_{\varepsilon} \delta \bar{\varepsilon}\right) \mathrm{d} \Omega-\int_{\Omega} \mathrm{R}^{\mathrm{iT}} \mathrm{N}_{\mathrm{u}^{\delta}} \mathrm{u} \mathrm{d} \Omega-\int_{\Gamma \mathrm{r}} \mathrm{r}^{\mathrm{iT}} \mathrm{N}_{\mathrm{u}^{\delta}} \mathrm{u} \mathrm{d} \Gamma
\end{aligned}
$$

As condições ótimas do problema 5.15 são:

$$
\begin{aligned}
& \overline{\mathrm{L}} \delta \mathrm{u}+\overline{\mathrm{C}}^{\mathrm{T}} \delta \bar{\sigma}=\overline{\mathrm{F}}^{\mathrm{i}} \\
& \delta \bar{\sigma}=\overline{\widetilde{\mathrm{E}}}_{\mathrm{n}} \delta \bar{\varepsilon} \\
& \overline{\mathrm{C}} \delta \mathrm{u}=\delta \bar{\varepsilon}
\end{aligned}
$$

onde os seguintes vetores e matrizes generalizados são definidos:

$$
\begin{aligned}
& \bar{L} \equiv \int_{\Omega} \rho{ }^{*} \mathrm{~N}_{\mathrm{u}}^{\mathrm{T}} \mathrm{N}_{\mathrm{u}} \mathrm{d} \Omega ; \overline{\mathrm{C}} \equiv \int_{\Omega} \mathrm{N}_{\sigma}^{\mathrm{T}} \mathrm{CN}_{\mathrm{u}} \mathrm{d} \Omega ; \\
& \mathrm{F}^{\mathrm{i}} \equiv \int_{\Omega} \mathrm{N}_{\mathrm{u}}^{\mathrm{T}} \mathrm{R}^{\mathrm{i}} \mathrm{d} \Omega+\int_{\Gamma_{\mathrm{r}}} \mathrm{N}_{\mathrm{u}}^{\mathrm{T}} \mathrm{r}^{\mathrm{i}} \mathrm{d} r ; \\
& \widetilde{\mathrm{E}}_{\mathrm{n}} \equiv \widetilde{\mathrm{E}}\left(\overline{\mathrm{D}}_{\mathrm{n}}\right) \equiv \int_{\Omega}\left(1-\mathrm{N}_{\mathrm{D}} \overline{\mathrm{D}}_{\mathrm{n}}\right)^{2} \mathrm{~N}_{\varepsilon}^{\mathrm{T}} \mathrm{EN}_{\varepsilon} \mathrm{d} \Omega ;
\end{aligned}
$$


L, $\rho$, F e fforam definidos no item 5.2. As equações 5.15-5.17 governam o problema discreto de previsão. As equações 5.15 e 5.17 expressam o equilíbrio dinâmico e a compatibilidade na forma fraca, enquanto a equação 5.16 é a lei de Hooke discretizada.

\subsection{Fase de correção}

O funcional da fase de correção, mostrado na equação 5.12, discretizado:

$$
\begin{aligned}
& \min _{\Delta \overline{\mathrm{D}}}\left\{\overline{\mathbf{L}}_{\mathbf{c}}\right\} \operatorname{com} \Delta \overline{\mathrm{D}} \geq \mathbf{0} \\
& \overline{\mathbf{L}}_{\mathbf{c}} \equiv \int_{\Omega} \frac{1}{2}\left(1-\mathrm{N}_{\mathrm{D}}\left(\overline{\mathrm{D}}_{\mathrm{n}}+\Delta \overline{\mathrm{D}}\right)\right)^{2}-\bar{\varepsilon}^{\mathrm{T}} \mathrm{N}_{\varepsilon}^{\mathrm{T}} \mathrm{EN}_{\varepsilon} \bar{\varepsilon}-2\left(\mathrm{~b}_{\mathrm{q}} \mathrm{P}^{\mathrm{T}} \mathrm{N}_{\varepsilon} \bar{\varepsilon}-\mathrm{b}_{4}\right) \mathrm{N}_{\mathrm{D}}\left(\overline{\mathrm{D}}_{\mathrm{n}}+\Delta \overline{\mathrm{D}}\right) \mathrm{d} \Omega \\
& -\int_{\Omega}\left(\mathrm{b}_{\mathrm{q}}\left(\mathrm{b}_{2}-1\right) \mathrm{P}^{\mathrm{T}} \mathrm{N}_{\varepsilon} \bar{\varepsilon}-\mathrm{b}_{3}\right)\left(\mathrm{N}_{\mathrm{D}}\left(\overline{\mathrm{D}}_{\mathrm{n}}+\Delta \overline{\mathrm{D}}\right)\right)^{2} \mathrm{~d} \Omega+\int_{\Omega} \frac{2}{3} \mathrm{~g}_{\mathrm{g}} \mathrm{b}_{2} \mathrm{P}^{\mathrm{T}} \mathrm{N}_{\varepsilon} \bar{\varepsilon}\left(\mathrm{N}_{\mathrm{D}}\left(\overline{\mathrm{D}}_{\mathrm{n}}+\Delta \overline{\mathrm{D}}\right)\right)^{3} \mathrm{~d} \Omega \\
& +\int_{\Omega} \mathrm{G} \nabla\left(\mathrm{N}_{\mathrm{D}}\left(\overline{\mathrm{D}}_{\mathrm{n}}+\Delta \overline{\mathrm{D}}\right)\right)^{\mathrm{T}} \nabla\left(\mathrm{N}_{\mathrm{D}}\left(\overline{\mathrm{D}}_{\mathrm{n}}+\Delta \overline{\mathrm{D}}\right)\right) \mathrm{d} \Omega
\end{aligned}
$$

As condições ótimas de Kuhn-Tucker do problema 5.20 são:

$$
\begin{aligned}
& \overline{\mathrm{f}} \equiv \bar{\varphi}(\bar{\varepsilon})-(\overline{\mathrm{a}}(\bar{\varepsilon})+\overline{\mathrm{D}}+\mathrm{C})\left(\mathrm{D}_{\mathrm{n}}+\Delta \mathrm{D}\right)-\overline{\mathrm{d}}\left(\bar{\varepsilon}, \mathrm{D}_{\mathrm{n}}+\Delta \mathrm{D}\right)-\overline{\mathrm{K}} \leq 0, \\
& \Delta \overline{\mathrm{D}} \geq 0, \quad \mathrm{f}^{\mathrm{T}} \Delta \overline{\mathrm{D}}=0
\end{aligned}
$$

onde os seguintes vetores e matrizes generalizados são definidos:

$$
\begin{aligned}
& \bar{\varphi}(\bar{\varepsilon}) \equiv \int_{\Omega} \mathrm{N}_{\mathrm{D}}^{\mathrm{T}}\left(\frac{1}{2} \bar{\varepsilon}^{\mathrm{T}} \mathrm{N}_{\varepsilon}^{\mathrm{T}} \mathrm{EN}_{\varepsilon} \bar{\varepsilon}+\mathrm{b}_{1} \mathrm{P}^{\mathrm{T}} \mathrm{N}_{\mathrm{e}} \bar{\varepsilon}\right) \mathrm{d} \Omega ; \\
& \mathrm{a}(\bar{\varepsilon}) \equiv \int_{\Omega}\left(\frac{1}{2} \bar{\varepsilon}^{\mathrm{T}} \mathrm{N}_{\varepsilon}^{\mathrm{T}} \mathrm{EN}_{\varepsilon} \bar{\varepsilon}-\mathrm{b}_{1}\left(\mathrm{~b}_{2}-1\right) \mathrm{P}^{\mathrm{T}} \mathrm{N}_{\varepsilon} \bar{\varepsilon}\right) \mathrm{N}_{\mathrm{D}}^{\mathrm{T}} \mathrm{N}_{\mathrm{D}} \mathrm{d} \Omega ; \\
& \overline{\mathrm{b}} \equiv \int_{\Omega} \mathrm{b}_{3} \mathrm{~N}_{\mathrm{D}}^{\mathrm{T}} \mathrm{N}_{\mathrm{D}} \mathrm{d} \Omega ; \\
& \mathrm{C} \equiv \int_{\Omega} \mathrm{G}\left(\nabla \mathrm{N}_{\mathrm{D}}\right)^{\mathrm{T}} \nabla \mathrm{N}_{\mathrm{D}} \mathrm{d} \Omega ; \overline{\mathrm{k}} \equiv \int_{\Omega} \mathrm{N}_{\mathrm{D}}^{\mathrm{T}} \mathrm{k} \mathrm{d} \Omega ; \\
& \mathrm{d}\left(\bar{\varepsilon}, \overline{\mathrm{D}}_{\mathrm{n}}+\Delta \overline{\mathrm{D}}\right) \equiv \int_{\Omega} \mathrm{b}_{2} \mathrm{P}_{2} \mathrm{P}^{\mathrm{T}} \mathrm{N}_{\varepsilon} \bar{\varepsilon} \mathrm{N}_{\mathrm{D}}^{\mathrm{T}}\left(\overline{\mathrm{D}}_{\mathrm{n}}+\Delta \overline{\mathrm{D}}\right)^{\mathrm{T}} \mathrm{N}_{\mathrm{D}}^{\mathrm{T}} \mathrm{N}_{\mathrm{D}}\left(\overline{\mathrm{D}}_{\mathrm{n}}+\Delta \overline{\mathrm{D}}\right) \mathrm{d} \Omega
\end{aligned}
$$


Para o caso de um parâmetro de difusão não constante (conforme equação 5.11), optou-se por $\mathrm{b}_{1}=\mathrm{b}_{2}=0$, e $\mathrm{b}_{3}=\mathrm{b}$. 0 funcional discretizado obtido é análogo ao anterior e, por brevidade, expõe-se aqui somente as matrizes finais:

$$
\begin{aligned}
& \bar{\varphi}(\bar{\varepsilon}) \equiv \int_{\Omega} \mathrm{N}_{\mathrm{D}}^{\mathrm{T}}\left(\frac{1}{2}-\bar{\varepsilon}^{\mathrm{T}} \mathrm{N}_{\varepsilon}^{\mathrm{T}} \mathrm{EN}_{\varepsilon} \bar{\varepsilon}\right) \mathrm{d} \Omega ; \\
& \mathrm{a}(\bar{\varepsilon}) \equiv \int_{\Omega}\left(\frac{(1+\mathrm{a})}{2} \bar{\varepsilon}-\mathrm{T} \mathrm{N}_{\varepsilon}^{\mathrm{T}} \mathrm{EN}_{\varepsilon} \bar{\varepsilon}\right) \mathrm{N}_{\mathrm{D}}^{\mathrm{T}} \mathrm{N}_{\mathrm{D}} \mathrm{d} \Omega ; \\
& \overline{\mathrm{b}} \equiv \int_{\Omega}(\mathrm{b}-\mathrm{ak}) \mathrm{N}_{\mathrm{D}}^{\mathrm{T}} \mathrm{N}_{\mathrm{D}} \mathrm{d} \Omega ; \\
& \mathrm{C} \equiv \int_{\Omega} \mathrm{G}\left(\nabla \mathrm{N}_{\mathrm{D}}\right)^{\mathrm{T}} \nabla \mathrm{N}_{\mathrm{D}} \mathrm{d} \Omega ; \mathrm{k} \equiv \int_{\Omega} \mathrm{N}_{\mathrm{D}}^{\mathrm{T}} \mathrm{kd} \Omega ; \\
& \overline{\mathrm{d}}\left(\bar{\varepsilon}, \overline{\mathrm{D}}_{\mathrm{n}}+\Delta \overline{\mathrm{D}}\right) \equiv \int_{\Omega} \mathrm{a}\left(\frac{1}{2} \bar{\varepsilon}-\mathrm{T} \mathrm{N}_{\varepsilon}^{\mathrm{T}} \mathrm{EN}_{\varepsilon} \bar{\varepsilon}-\mathrm{b}\right) \mathrm{N}_{\mathrm{D}}^{\mathrm{T}}\left(\overline{\mathrm{D}}_{\mathrm{n}}+\Delta \overline{\mathrm{D}}\right)^{\mathrm{T}} \mathrm{N}_{\mathrm{D}}^{\mathrm{T}} \mathrm{N}_{\mathrm{D}}\left(\overline{\mathrm{D}}_{\mathrm{n}}+\Delta \overline{\mathrm{D}}\right) \mathrm{d} \Omega
\end{aligned}
$$

de acordo com a equação 5.21a.

\subsubsection{Sobre implementação}

Note que, devido à necessidade de continuidade na interpolação das funções $\mathrm{N}_{\mathrm{D}}$ e $\mathrm{N}_{\mathrm{u}}$, as matrizes a, $\bar{b}$ e c exibem uma estrutura de banda, mas não diagonal. Portanto, a lei constitutiva em variáveis generalizadas não pode ser imposta em um ponto específico, mas deve referir-se a toda a estrutura discretizada. 0 problema é facilmente resolvido, uma vez que 0 conjunto de equações 5.21 tem a forma de um problema de complementariedade não - linear (PCNL). Caso $\bar{d}=0$, tem-se 0 problema de complementariedadeliner(PCL) e a solução é menos complexa.

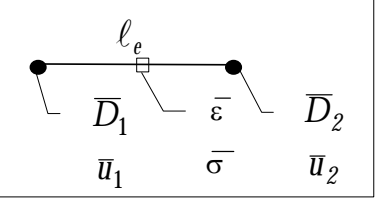

$$
\begin{aligned}
& \mathrm{N}_{\varepsilon}^{\mathrm{e}}=1 ; \quad \mathrm{N}_{\sigma}^{\mathrm{e}}=\frac{1}{\Omega^{\mathrm{e}}} ; \\
& \mathrm{N}_{\mathrm{u}}^{\mathrm{e}}=\mathrm{N}_{\mathrm{D}}^{\mathrm{e}}=\left[\left(1-\frac{\mathrm{x}}{\ell^{\mathrm{e}}}\right) \frac{\mathrm{x}}{\ell^{\mathrm{e}}}\right] \text {; } \\
& \overline{\mathrm{L}}^{\mathrm{e}}=\frac{\rho \Omega^{\mathrm{e}}}{6}\left[\begin{array}{ll}
2 & 1 \\
1 & 2
\end{array}\right] ; \quad \overline{\mathrm{C}}^{\mathrm{e}}=\left[\begin{array}{ll}
-\frac{1}{\ell^{\mathrm{e}}} & \frac{1}{\ell^{\mathrm{e}}}
\end{array}\right] ; \\
& \widetilde{\mathrm{E}}^{\mathrm{e}}=\Omega{ }^{\mathrm{e}} \mathrm{E}\left\{1+\frac{1}{3}\left(\overline{\mathrm{D}}_{1}^{2}+\overline{\mathrm{D}}_{2}^{2}+\overline{\mathrm{D}}_{1} \overline{\mathrm{D}}_{2}\right)-\left(\overline{\mathrm{D}}_{1}+\overline{\mathrm{D}}_{2}\right)\right\}
\end{aligned}
$$


Adota-se a solução para PCL sugerida em Mangasarian ${ }^{58}$ e discutida em Pang ${ }^{59}$ e Nor $\&$ Zarae ${ }^{60}$, e utiliza-se 0 programa Path para PCNL. Path é um programa desenvolvido por Dirkse\& Femis ${ }^{61}$ em Linguagem C, e cedido pelo prof. D r. Michael C. Ferris, da Universidade de Wisconsin.

As expressões explícitas das funções de interpolação e as matrizes e vetores generalizados válidos para os todos os modelos A, B, C, estão na Figura 5.2. Os Quadros 5.1 e 5.2 são particularizados para os modelos A,B e C, respectivamente.

$$
\begin{aligned}
& \bar{\varphi}^{\mathrm{e}}=\frac{\Omega^{\mathrm{e}}\left(\mathrm{E}^{-2}+2 \mathrm{~b}^{-} \bar{\varepsilon}\right)}{4}\left\{\begin{array}{l}
1 \\
1
\end{array} ; \quad \mathrm{k}^{\mathrm{e}}=\frac{\Omega}{2} \mathrm{k}\left\{\begin{array}{l}
1 \\
1
\end{array}\right\} ;\right. \\
& \mathrm{a}^{\mathrm{e}}=\frac{\Omega^{\mathrm{e}}\left[\mathrm{E}^{-2}-2 \mathrm{q}\left(\mathrm{b}_{2}-1\right) \bar{\varepsilon}\right]}{12}\left[\begin{array}{ll}
2 & 1 \\
1 & 2
\end{array}\right] ; \\
& \overline{\mathrm{b}}^{\mathrm{e}}=\frac{\mathrm{b}_{3}}{6}\left[\begin{array}{ll}
2 & 1 \\
1 & 2
\end{array}\right] ; \quad \mathrm{c}^{\mathrm{e}}=\frac{\Omega{ }^{\mathrm{e}} \mathrm{G}}{\ell^{\mathrm{e}}}\left[\begin{array}{cc}
1 & -1 \\
-1 & 1
\end{array}\right] ; \\
& \overline{\mathrm{d}}^{\mathrm{e}}=\frac{\mathrm{g}_{2} \mathrm{~b}_{2}}{2} \bar{\varepsilon}\left\{\begin{array}{l}
\overline{\mathrm{D}}_{1}^{2}+\frac{2}{3} \overline{\mathrm{D}}_{1} \overline{\mathrm{D}}_{2}+\frac{1}{3} \overline{\mathrm{D}}_{2}^{2} \\
\frac{1}{3} \overline{\mathrm{D}}_{1}^{2}+\frac{2}{3} \overline{\mathrm{D}}_{1} \overline{\mathrm{D}}_{2}+\overline{\mathrm{D}}_{2}^{2}
\end{array}\right\}
\end{aligned}
$$

Quadro 5.1. Matrizes e vetores de variáveis generalizadas do elemento TRU-2, para 0 caso $b_{1} b_{2} \neq 0$.

$$
\begin{aligned}
& \bar{\varphi}^{\mathrm{e}}=\frac{\Omega^{\mathrm{e}} \mathrm{E}^{-2}}{4}\left\{\begin{array}{l}
1 \\
1
\end{array}\right] ; \quad \mathrm{k}^{\mathrm{e}}=\frac{\Omega}{2} \mathrm{k}\left\{\begin{array}{l}
1 \\
1
\end{array}\right\} ; \\
& \mathrm{a}^{\mathrm{e}}=(1+\mathrm{a}) \frac{\Omega^{\mathrm{e}} \mathrm{E}^{-2}}{12}\left[\begin{array}{ll}
2 & 1 \\
1 & 2
\end{array}\right] ; \\
& \overline{\mathrm{b}}^{\mathrm{e}}=\frac{(\mathrm{b}-\mathrm{ak})}{6}\left[\begin{array}{ll}
2 & 1 \\
1 & 2
\end{array}\right] ; \quad \mathrm{C}^{\mathrm{e}}=\frac{\Omega^{\mathrm{e}}}{\ell^{\mathrm{e}}}\left[\begin{array}{cc}
1 & -1 \\
-1 & 1
\end{array}\right] ; \\
& \mathrm{d}^{\mathrm{e}}=\mathrm{a} \frac{\left(\mathrm{E}^{-2}-2 \mathrm{~b}\right)}{4}\left\{\begin{array}{l}
\overline{\mathrm{D}}_{1}^{2}+\frac{2}{3} \overline{\mathrm{D}}_{1} \overline{\mathrm{D}}_{2}+\frac{1}{3} \overline{\mathrm{D}}_{2}^{2} \\
\frac{1}{3} \overline{\mathrm{D}}_{1}^{2}+\frac{2}{3} \overline{\mathrm{D}}_{1} \overline{\mathrm{D}}_{2}+\overline{\mathrm{D}}_{2}^{2}
\end{array}\right\}
\end{aligned}
$$

Quadro 5.2. Matrizes e vetores de variáveis generalizadas do elemento TRU-2, para 0 caso a $\neq 0$.

\subsubsection{Elemento CST-3}

O elemento CST-3 é um elemento triangular com deformação plana, interpolação constante para tensões e deformações, e interpolação linear para deslocamentos e dano. Este se refere ao modelo de dano com gradiente de segunda ordem, com variável isotrópica de dano. 
5.5.3.1 Discretização do funcional

\subsection{Fase de previsão}

A formulação é a mesma apresentada para o elemento TRU-2.

\subsection{Fase de correção}

A forma discretizada do problema de correção é dada novamente, por um PCNL:

$$
\begin{aligned}
& \overline{\mathrm{f}} \equiv \bar{\varphi}(\bar{\varepsilon})-(\overline{\mathrm{a}}(\bar{\varepsilon})+\overline{\mathrm{b}}+\mathrm{C})\left(\overline{\mathrm{D}}_{\mathrm{n}}+\Delta \overline{\mathrm{D}}\right)-\overline{\mathrm{d}}\left(\bar{\varepsilon}, \overline{\mathrm{D}}_{\mathrm{n}}+\Delta \overline{\mathrm{D}}\right)-\overline{\mathrm{k}} \leq 0, \\
& \Delta \mathrm{D} \geq 0, \quad \mathrm{f}^{\mathrm{T}} \Delta \mathrm{D}=0
\end{aligned}
$$

O s vetores e matrizes foram definidos considerando os casos: $\mathrm{a}=0$ e $\mathrm{b}_{1} \mathrm{~b}_{2} \neq 0$, e $\mathrm{a} \neq 0$ e $\mathrm{b}_{1} \mathrm{~b}_{2}=0$, respectivamente:

$$
\begin{aligned}
& \bar{\varphi}(\bar{\varepsilon}) \equiv \int_{\Omega} \mathrm{N}_{\mathrm{D}}^{\mathrm{T}}\left(\frac{1}{2}-\bar{\varepsilon}^{\mathrm{T}} \mathrm{N}_{\varepsilon}^{\mathrm{T}} \mathrm{EN}_{\varepsilon} \bar{\varepsilon}+\mathrm{bP}^{\mathrm{T}} \mathrm{N}_{\varepsilon} \bar{\varepsilon}\right) \mathrm{d} \Omega ; \\
& \mathrm{a}(\bar{\varepsilon}) \equiv \int_{\Omega}\left(\frac{1}{2} \bar{\varepsilon}^{\mathrm{T}} \mathrm{N}_{\varepsilon}^{\mathrm{T}} \mathrm{EN}_{\varepsilon} \bar{\varepsilon}-\mathrm{b}\left(\mathrm{b}_{2}-1\right) \mathrm{P}^{\mathrm{T}} \mathrm{N}_{\varepsilon} \bar{\varepsilon}\right) \mathrm{N}_{\mathrm{D}}^{\mathrm{T}} \mathrm{N}_{\mathrm{D}} \mathrm{d} \Omega ; \\
& \overline{\mathrm{b}} \equiv \int_{\Omega} \mathrm{b}_{3} \mathrm{~N}_{\mathrm{D}}^{\mathrm{T}} \mathrm{N}_{\mathrm{D}} \mathrm{d} \Omega ; \quad \mathrm{C} \equiv \int_{\Omega} \mathrm{G}\left(\nabla \mathrm{N}_{\mathrm{D}}\right)^{\mathrm{T}} \nabla \mathrm{N}_{\mathrm{D}} \mathrm{d} \Omega ; \quad \overline{\mathrm{K}} \equiv \int_{\Omega} \mathrm{N}_{\mathrm{D}}^{\mathrm{T}} \mathrm{k} \mathrm{d} \Omega ; \\
& \overline{\mathrm{d}}\left(\bar{\varepsilon}, \overline{\mathrm{D}}_{\mathrm{n}}+\Delta \overline{\mathrm{D}}\right) \equiv \int_{\Omega} \mathrm{g}_{2} \mathrm{P}^{\mathrm{T}} \mathrm{N}_{\varepsilon} \bar{\varepsilon} \mathrm{N}_{\mathrm{D}}^{\mathrm{T}}\left(\overline{\mathrm{D}}_{\mathrm{n}}+\Delta \overline{\mathrm{D}}\right)^{\mathrm{T}} \mathrm{N}_{\mathrm{D}}^{\mathrm{T}} \mathrm{N}_{\mathrm{D}}\left(\overline{\mathrm{D}}_{\mathrm{n}}+\Delta \overline{\mathrm{D}}\right) \mathrm{d} \Omega \\
& \bar{\varphi}(\bar{\varepsilon}) \equiv \int_{\Omega} \mathrm{N}_{\mathrm{D}}^{\mathrm{T}}\left(\frac{1}{2} \bar{\varepsilon}-\mathrm{T} \mathrm{N}_{\varepsilon}^{\mathrm{T}} \mathrm{EN}_{\varepsilon} \bar{\varepsilon}+\mathrm{b}_{\mathrm{1}} \mathrm{P}^{\mathrm{T}} \mathrm{N}_{\varepsilon} \bar{\varepsilon}\right) \mathrm{d} \Omega ; \\
& \mathrm{a}(\bar{\varepsilon}) \equiv \int_{\Omega}\left(\frac{(1+\mathrm{a})}{2}-\bar{\varepsilon}-\mathrm{N}_{\varepsilon}^{\mathrm{T}} \mathrm{EN}_{\varepsilon} \bar{\varepsilon}\right) \mathrm{N}_{\mathrm{D}}^{\mathrm{T}} \mathrm{N}_{\mathrm{D}} \mathrm{d} \mathrm{\alpha} ; \\
& \overline{\mathrm{b}} \equiv \int_{\Omega}\left(\mathrm{b}_{3}-\mathrm{ak}\right) \mathrm{N}_{\mathrm{D}}^{\mathrm{T}} \mathrm{N}_{\mathrm{D}} \mathrm{d} 2 ; \mathrm{C} \equiv \int_{\Omega} \mathrm{G}\left(\nabla \mathrm{N}_{\mathrm{D}}\right)^{\mathrm{T}} \nabla \mathrm{N}_{\mathrm{D}} \mathrm{d} \Omega ; \mathrm{k} \equiv \int_{\Omega} \mathrm{N}_{\mathrm{D}}^{\mathrm{T}} \mathrm{kd} 2 ; \\
& \overline{\mathrm{d}}\left(\bar{\varepsilon}, \overline{\mathrm{D}}_{\mathrm{n}}+\Delta \overline{\mathrm{D}}\right) \equiv \int_{\Omega} \mathrm{a}\left(\frac{1}{2} \bar{\varepsilon}^{\mathrm{T}} \mathrm{N}_{\varepsilon}^{\mathrm{T}} \mathrm{EN}_{\varepsilon} \bar{\varepsilon}-\mathrm{b}_{3}\right) \mathrm{P}^{\mathrm{T}} \mathrm{N}_{\mathrm{D}}^{\mathrm{T}}\left(\overline{\mathrm{D}}_{\mathrm{n}}+\Delta \overline{\mathrm{D}}\right)^{\mathrm{T}} \mathrm{N}_{\mathrm{D}}^{\mathrm{T}} \mathrm{N}_{\mathrm{D}}\left(\overline{\mathrm{D}}_{\mathrm{n}}+\Delta \overline{\mathrm{D}}\right) \mathrm{d} \Omega
\end{aligned}
$$


No caso, enquanto as matrizes e vetores 5.25 consideram os parâmetros de dissimetria do modelo $\mathrm{b}_{1}$ e $\mathrm{b}_{2}$, em 5.26 permite-se a utilização do parâmetro de difusão não constante $\mathrm{a} \neq 0$.

\subsubsection{Sobre a implementação}

A Figura 5.3 mostra o desenho do elemento, as funções de forma adotadas e as matrizes da fase de previsão explícitas.

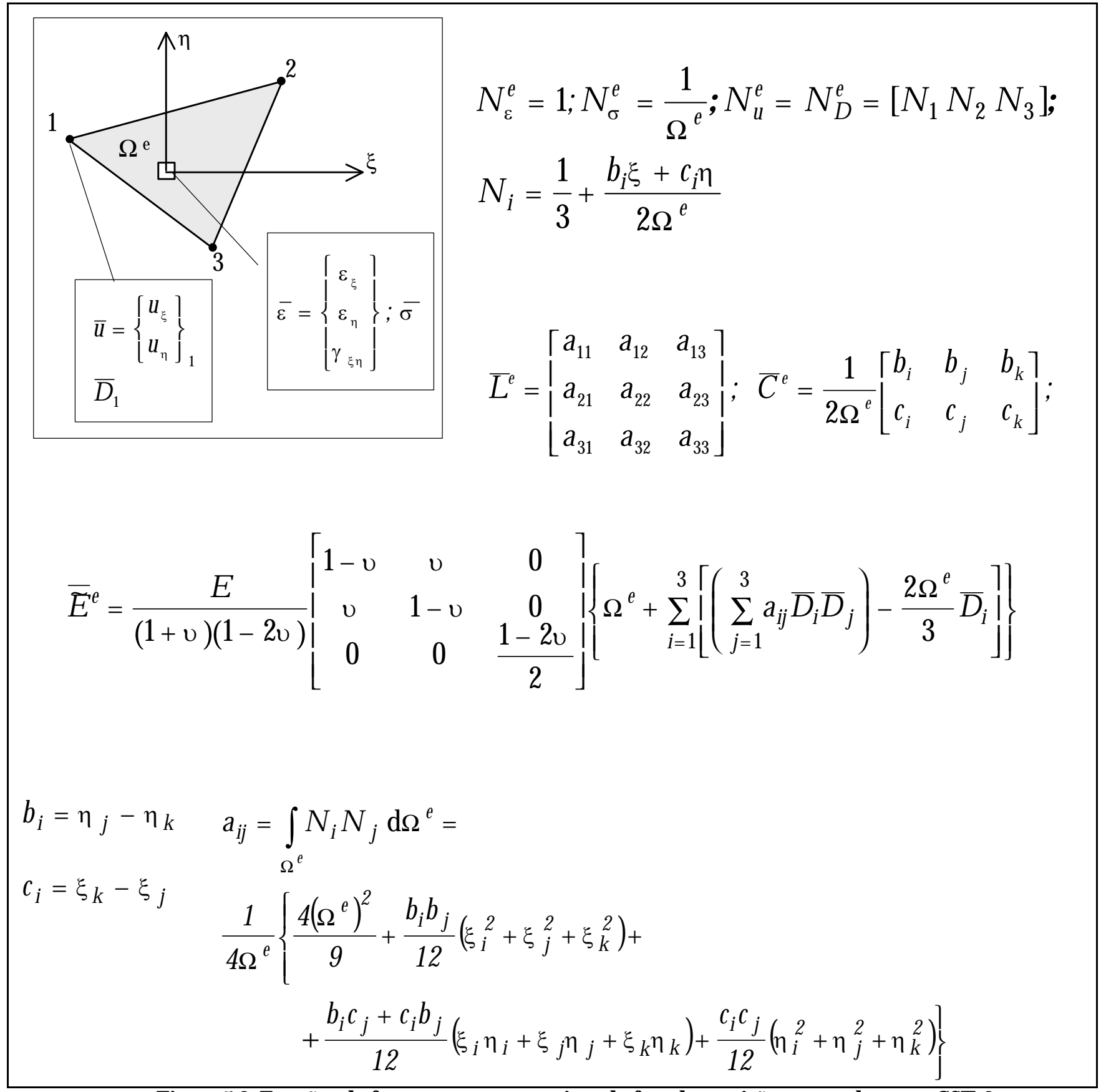

Figura 5.3. Funções de forma, vetores e matrizes da fase de previsão para o elemento CST -3. 
Os Quadros 5.3 e 5.4 mostram os vetores e matrizes explícitas da fase de correção, para as formulações 5.25 e 5.26, respectivamente.

$$
\begin{aligned}
& \bar{\varphi}^{\mathrm{e}}=\frac{\Omega^{\mathrm{e}}{ }_{\varepsilon}^{\mathrm{T}} \mathrm{E} \varepsilon+2 \mathrm{~b}_{1} \mathrm{P}^{\mathrm{T}} \varepsilon}{6}\left\{\begin{array}{l}
1 \\
1 \\
1
\end{array}\right\} ; \mathrm{a}^{\mathrm{e}}=\frac{{ }^{\mathrm{T}} \mathrm{E} \varepsilon-\mathrm{b}_{1}\left(\mathrm{~b}_{2}-1\right) \mathrm{P}^{\mathrm{T}} \varepsilon}{2}\left[\begin{array}{lll}
\mathrm{a}_{11} & \mathrm{a}_{12} & \mathrm{a}_{13} \\
\mathrm{a}_{21} & \mathrm{a}_{22} & \mathrm{a}_{23} \\
\mathrm{a}_{31} & \mathrm{a}_{32} & \mathrm{a}_{33}
\end{array}\right] \\
& \left.\bar{b}^{\mathrm{e}}=\mathrm{b}_{3}\left[\begin{array}{ccc}
\mathrm{a}_{11} & \mathrm{a}_{12} & \mathrm{a}_{13} \\
\mathrm{a}_{21} & \mathrm{a}_{22} & \mathrm{a}_{23} \\
\mathrm{a}_{31} & \mathrm{a}_{32} & \mathrm{a}_{33}
\end{array}\right] ; \mathrm{c}^{\mathrm{e}}=\frac{\mathrm{c}}{4 \Omega^{\mathrm{e}}} \mid \begin{array}{ccc}
\mathrm{b}_{1}^{2}+\mathrm{c}_{1}^{2} & \mathrm{~b}_{1} \mathrm{~b}_{2}+\mathrm{c}_{\mathrm{c}} & \mathrm{b}_{1} \mathrm{~b}_{3}+\mathrm{c}_{1} \mathrm{c}_{3} \\
& \mathrm{~b}_{2}^{2}+\mathrm{c}_{2}^{2} & \mathrm{~b}_{2} \mathrm{~b}_{3}+\mathrm{c}_{2} \mathrm{c}_{3} \\
\operatorname{sim} & & \mathrm{b}_{3}^{2}+\mathrm{c}_{3}^{2}
\end{array}\right]
\end{aligned}
$$

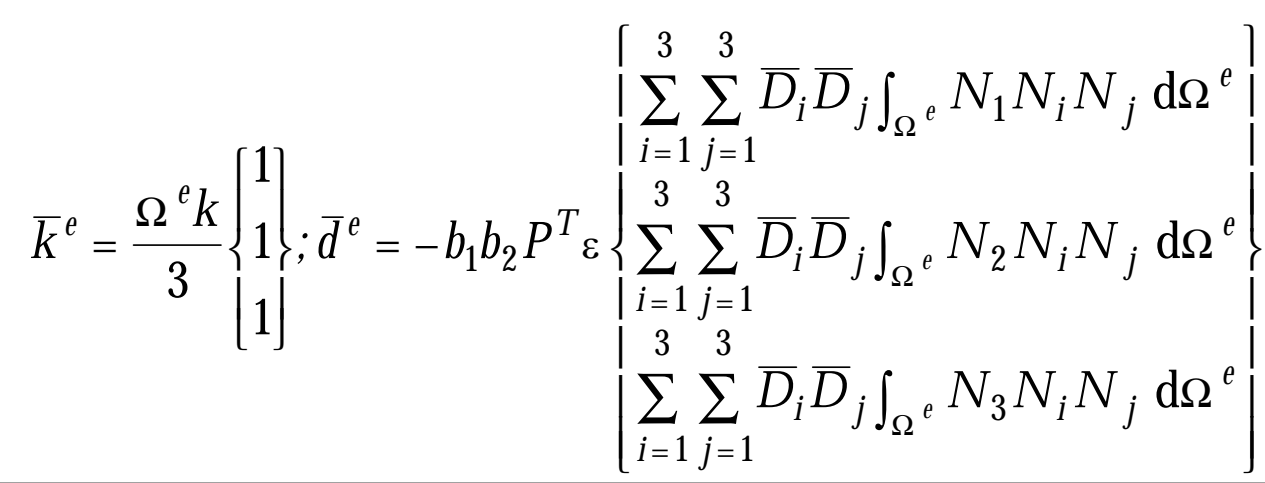

Quadro 5.3. Vetores e matrizes de variáveis generalizadas do elemento CST -3, para 0 caso $b_{1} b_{2} \neq 0$.

$$
\begin{aligned}
& \bar{\varphi}^{\mathrm{e}}=\frac{\Omega^{\mathrm{e}}{ }^{\mathrm{T}} \mathrm{E} \varepsilon}{6}\left\{\begin{array}{l}
1 \\
1 \\
1
\end{array}\right\} ; \mathrm{a}^{\mathrm{e}}=\frac{(1+\mathrm{a}) \varepsilon}{2} \mathrm{E}_{\varepsilon}\left[\begin{array}{lll}
\mathrm{a}_{11} & \mathrm{a}_{12} & \mathrm{a}_{13} \\
\mathrm{a}_{21} & \mathrm{a}_{22} & \mathrm{a}_{23} \\
\mathrm{a}_{31} & \mathrm{a}_{32} & \mathrm{a}_{33}
\end{array}\right] ;
\end{aligned}
$$

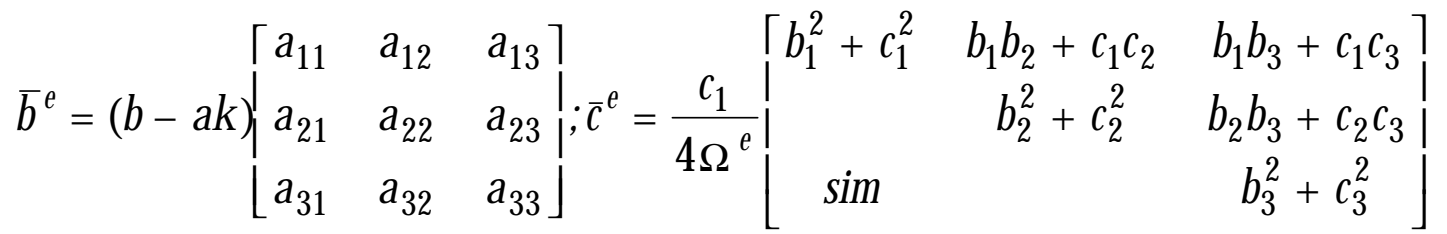

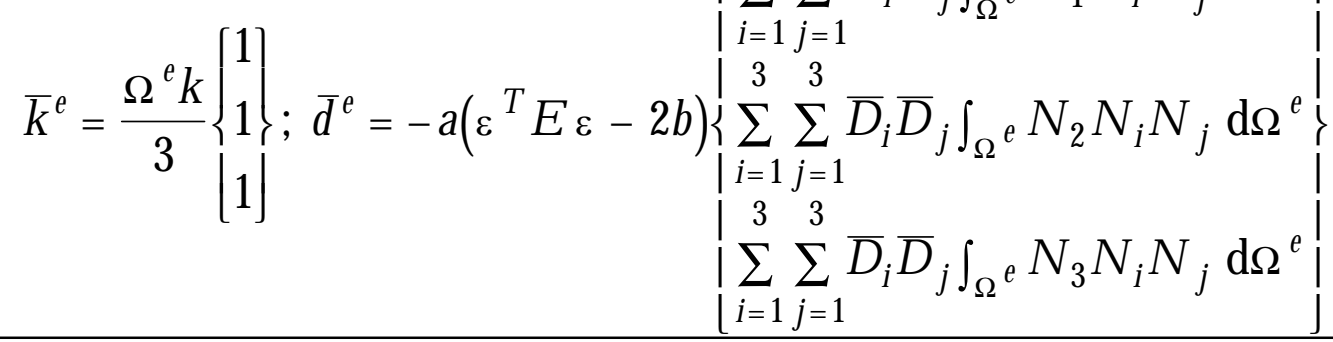

Q uadro 5.4. Vetores e matrizes de variáveis generalizadas do elemento CST -3, para 0 caso a $\neq 0$. 


\subsubsection{Elemento TRU-3}

Elemento TRU-3 é um elemento de barra com interpolação constante para tensões e deformações, interpolação linear para deslocamentos e variável de dano e interpolação parabólica para gradiente de dano. Este se refere ao modelo de dano com gradiente de segunda ordem, com variável isotrópica de dano.

D eve-se observar que o programa não permite que um exemplo apresente, ao mesmo tempo, gradientes segundo e quarto $\left(\mathrm{G}_{1}, \mathrm{C}_{2} \neq 0\right)$ com parâmetro de difusão não constante, $a \neq 0$. As implementações seguem os modelos $A, B$ e $C$ definidos no Capítulo 4.

\subsubsection{Discretização do variacional}

\subsection{Fase de previsão}

Para a fase de previsão, a forma discreta do problema é a mesma apresentada para 0 elemento TRU-2.

\subsection{Fase de correção}

Para a fase de correção, adaptou-se uma formulação mista (Comi \& Driemei $\left.{ }^{11}\right)$, de modo que apenas continuidade $\mathrm{C}^{0}$ é requerida para aproximação do campo de dano, mesmo quando termos em quarta ordem estão presentes no funcional.

A formulação variacional mista pode ser obtida introduzindo o gradiente de dano $\Theta$ como uma variável independente e adicionando ao funcional a restrição $\Theta=\nabla D$, ponderada por um multiplicador de Lagrange $\mu$.

O variacional discretizado:

$$
\begin{aligned}
& \min _{\Delta \overline{\mathbf{D}}, \bar{\Theta}} \max _{\bar{\mu}}\left\{\overline{\mathbf{L}}_{\mathbf{c}}^{*}\right\} \text { com } \Delta \overline{\mathrm{D}} \geq 0 \\
& \mathbf{L}_{\mathbf{c}}^{*}(\Delta \mathrm{D}, \Theta ; \mu) \equiv \int_{\Omega}\left[\frac{1}{2}\left(1-\mathrm{N}_{\mathrm{D}} \overline{\mathrm{D}}\right)^{2} \bar{\varepsilon}^{\mathrm{T}} \mathrm{E} \bar{\varepsilon}+2 \mathrm{k} \mathrm{N}_{\mathrm{D}} \mathrm{D}+\mathrm{b}_{3}\left(\mathrm{~N}_{\mathrm{D}} \overline{\mathrm{D}}\right)^{2}\right] \mathrm{d} \Omega \\
& \quad+\int_{\Omega} \mathrm{G}\left(\mathrm{N}_{\mathrm{D}} \bar{\Theta}\right)^{2} \mathrm{~d} \Omega+\int_{\Omega} \mathrm{G}_{2}\left(\nabla \mathrm{N}_{\mathrm{D}} \bar{\Theta}\right)^{\mathrm{T}}\left(\nabla \mathrm{N}_{\mathrm{D}} \bar{\Theta}\right) \mathrm{d} \Omega \\
& \quad+\int_{\Omega} 2\left(\mathrm{~N}_{\mu} \bar{\mu}\right)^{\mathrm{T}}\left(\nabla \mathrm{N}_{\mathrm{D}} \overline{\mathrm{D}}-\mathrm{N}_{\mathrm{D}} \bar{\Theta}\right) \mathrm{d} \Omega
\end{aligned}
$$


O PCNL da forma discreta da fase de correção:

$$
\begin{aligned}
& \overline{\mathrm{f}} \equiv \bar{\varphi}(\bar{\varepsilon})-(\overline{\mathrm{a}}(\bar{\varepsilon})+\overline{\mathrm{b}})\left(\overline{\mathrm{D}}_{\mathrm{n}}+\Delta \overline{\mathrm{D}}\right)+\overline{\mathrm{d}}\left(\bar{\varepsilon}, \overline{\mathrm{D}}_{\mathrm{n}}+\Delta \overline{\mathrm{D}}\right)-\overline{\mathrm{k}}-\mathrm{g}_{\bar{\mu}} \overline{-} 0 \\
& \Delta \overline{\mathrm{D}} \geq 0, \quad \mathrm{f}^{\mathrm{T}} \Delta \overline{\mathrm{D}}=0 \\
& \overline{\mathrm{q}} \bar{\Theta}+\overline{\mathrm{C}}_{2} \bar{\Theta}-\overline{\mathrm{h}} \bar{\mu}=0 \\
& \mathrm{~g}^{\mathrm{T}} \mathrm{D}-\overline{\mathrm{h}}^{\mathrm{T}} \bar{\Theta}=0
\end{aligned}
$$

onde:

$$
\begin{array}{ll}
\mathrm{g} \equiv \int_{\Omega}\left(\nabla \mathrm{N}_{\mathrm{D}}\right)^{\mathrm{T}} \mathrm{N}_{\mu} \mathrm{d} \Omega ; & \mathrm{G} \equiv \int_{\Omega} \mathrm{G}_{\Theta} \mathrm{N}_{\Theta}^{\mathrm{T}} \mathrm{N}_{\Theta} \mathrm{d} \Omega ; \\
\mathrm{G} \equiv \int_{\Omega} \mathrm{G}_{2}\left(\nabla \mathrm{N}_{\Theta}\right)^{\mathrm{T}} \nabla \mathrm{N}_{\Theta} \mathrm{d} \Omega ; & \mathrm{h} \equiv \int_{\Omega} \mathrm{N}_{\Theta}^{\mathrm{T}} \mathrm{N}_{\mu} \mathrm{d} \Omega
\end{array}
$$

Considera-se $n_{D}, n_{\Theta}$ e $n_{\mu}$ o número de variáveis generalizadas $\bar{D}, \bar{\Theta}$ e $\bar{\mu}$, respectivamente, considerando todo o agregado de elementos finitos e $n_{D}^{\mathrm{e}}, \mathrm{n}_{\Theta}^{\mathrm{e}}$ e $\mathrm{n}_{\mu}^{\mathrm{e}}$ o número das mesmas variáveis por elemento.

No modelamento do multiplicador lagrangiano $\mu$ é descontínuo através dos elementos, a equação 5.31 se desacopla a nível de elemento e pode ser usada para condensar tantos graus de liberdade $\bar{\Theta}$ dos pontos internos quanto o número de multiplicadores generalizados de Lagrange por elemento $\eta_{\mu}^{\mathrm{e}}$. Por exemplo, se $\mu$ é constante em um elemento $\mathrm{n}_{\mu}^{\mathrm{e}}=1$, como para o elemento TRU-3, uma variável generalizada interna $\bar{\Theta}_{\mathrm{i}}$ pode ser eliminada. Deste modo, as $\mathrm{n}_{\Theta}$ equações 5.30 permitem computar as $\mathrm{n}_{\mu}$ variáveis $\bar{\mu}$ e as $\left(\mathrm{n}_{\Theta}-\mathrm{n}_{\mu}\right)$ variáveis restantes $\bar{\Theta}$ como função de todas as variáveis $\overline{\mathrm{D}}$. D evido à necessidade de continuidade do campo aproximado $\Theta$, esta parte deve ser computada a nível estrutural, mas apenas uma vez na análise toda.

Finalmente, substituindo estes valores na função generalizada de escoamento 5.28 obtém-se a fase de correção discretizada, na forma de um problema de complementariedade não - linear, diretamente obtido do princípio da mínima energia:

$$
\begin{aligned}
& \overline{\mathrm{f}} \equiv \bar{\varphi}(\bar{\varepsilon})-(\overline{\mathrm{a}}(\bar{\varepsilon})+\overline{\mathrm{b}}) \overline{\mathrm{D}}+\overline{\mathrm{d}}(\bar{\varepsilon}, \overline{\mathrm{D}})-\overline{\mathrm{k}}-\overline{\mathrm{G}} \overline{\mathrm{D}} \leq 0, \\
& \Delta \overline{\mathrm{D}} \geq 0, \quad \mathrm{f}^{\mathrm{T}} \Delta \overline{\mathrm{D}}=0
\end{aligned}
$$


onde a matriz $\bar{G}$, que depende das matrizes de interpolação $N_{\Theta}, N_{D}$ e $N_{\mu}$, considera os termos gradientes.

O processo de solução do problema seguido neste trabalho para a fase de correção pode ser interpretado como 'Método de restrições discretas de Kirchhoff' (disaze Kirdhoff constraints approadh). Este método foi introduzido no contexto da elasticidade para formulação de elementos de viga, membrana e casca que permitem que se despreze a deformação por cisalhamento (conforme Zienkievicz \& Tayla ${ }^{55,56}$ ).

\subsection{Sobre a implementação}

Os vetores e matrizes para a formulação mista do elemento estão resumidos na Figura 5.4 .

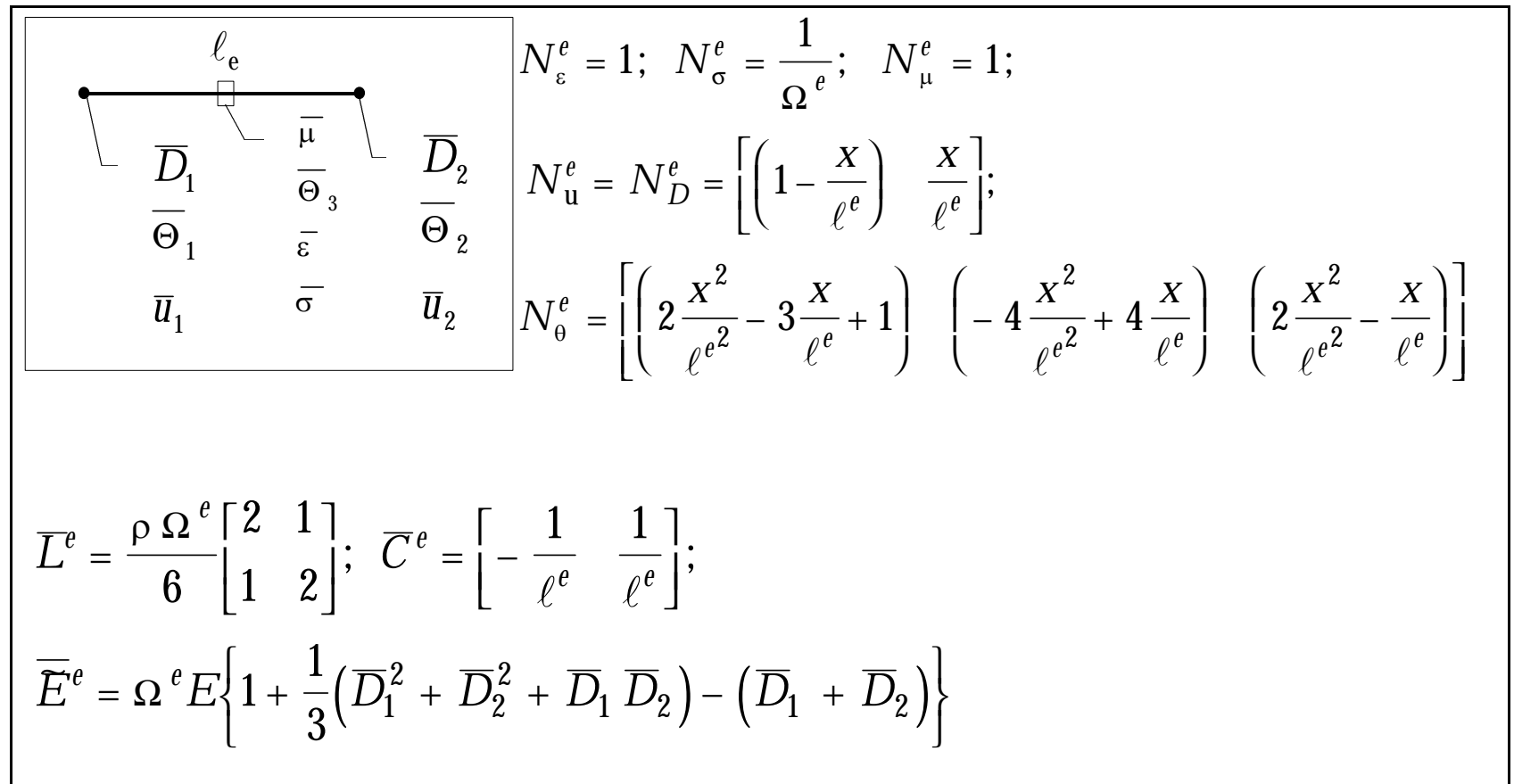

Figura 5.4. Elemento de viga: variáveis generalizadas, modelo de dano com gradiente de quarta ondem.

$\mathrm{O}$ vetor $\overline{\mathrm{G}}$, em função de $\mathrm{G}, \mathrm{C}_{2}, \mathrm{~N}_{\Theta}, \mathrm{N}_{\mathrm{D}}, \mathrm{N}_{\mu}$, não aparece na Figura 5.4, uma vez que é obtido com uma seqüência de cálculos, resumida no Quadro 5.5. 


$$
\begin{aligned}
& \left.\overline{\mathrm{A}}^{\mathrm{e}}=\frac{\Omega}{30}\left\{\frac{\mathrm{q}}{2} \mid \begin{array}{cc}
7 & -3 \\
-1 & -1 \\
-3 & 7
\end{array}\right]+\frac{10 \mathrm{c}_{2}}{\ell^{2}}\left[\begin{array}{cc}
9 & 3 \\
-12 & -12 \\
3 & 9
\end{array}\right]\right\} ; \overline{\mathrm{B}}^{\mathrm{e}}=-\frac{\Omega^{\mathrm{e}}}{6}\left\{\begin{array}{l}
1 \\
4 \\
1
\end{array}\right\}
\end{aligned}
$$

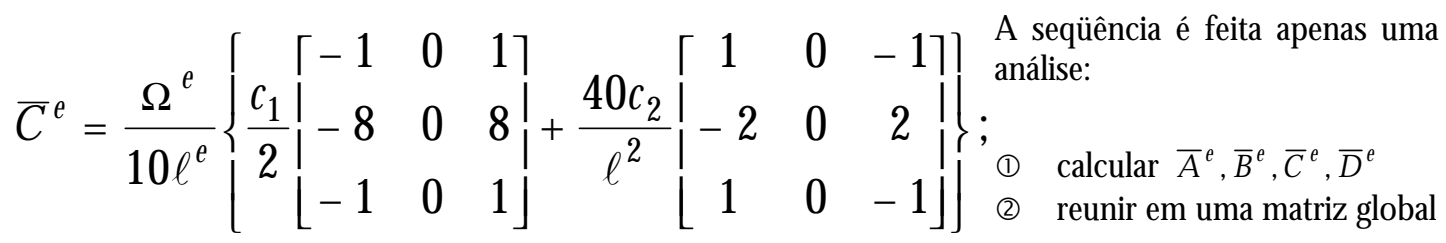

$$
\begin{aligned}
& \bar{D}^{\mathrm{e}}=-\frac{\Omega^{\mathrm{e}}}{\ell^{\mathrm{e}}}\left\{\begin{array}{lll}
-1 & 0 & 1
\end{array}\right\} \text {; } \\
& \mathrm{A}: \overline{\mathrm{A}}^{\mathrm{e}}, \overline{\mathrm{B}}^{\mathrm{e}} ; \mathrm{C}: \overline{\mathrm{C}}^{\mathrm{e}} \text { e } \mathrm{D}: \overline{\mathrm{D}}^{\mathrm{e}}
\end{aligned}
$$




\subsection{Introdução}

Neste capítulo serão apresentados um exemplo unidimensional e três exemplos bidimensionais, com o objetivo de mostrar as conseqüências numéricas do fenômeno da localização e a resposta obtida com o modelo de regularização. Os elementos finitos usados neste capítulo foram apresentados no Capítulo 5.

\subsection{Exemplo unidimensional}

\subsubsection{Exemplo teórico unidimensional}

Para o exemplo unidimensional, considere-se a discretização em 40 (malha 1), 80 (malha 2) e 160 (malha 3) elementos de uma barra dinamicamente tracionada, conforme Figura 6.1. Para integração dinâmica, a amplitude do passo de tempo é $\Delta \mathrm{t}=5 \times 10^{-7}$. Com relação à carga aplicada $\mathrm{F}(\mathrm{t}), \bar{\sigma}$ refere-se à tensão máxima, a partir da qual se inicia a danificação do material e o regime softening 


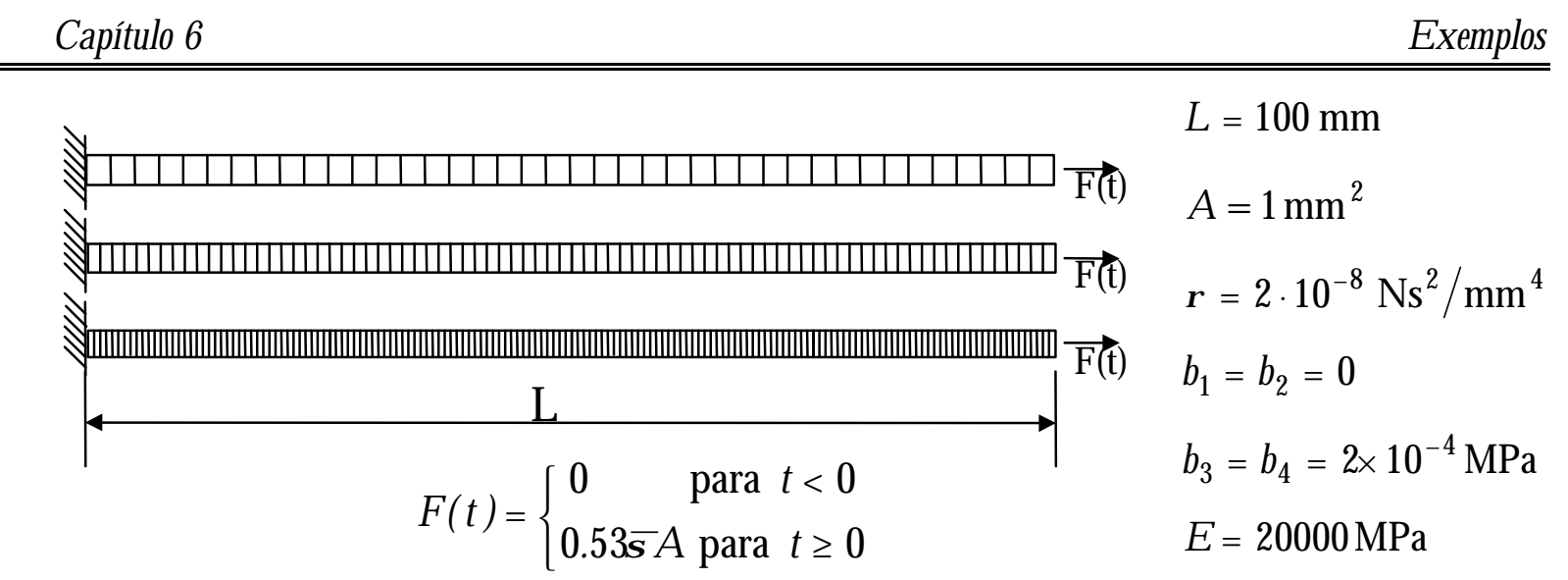

Figura 6.1. Exemplo unidimensional: dados geométricos, de carregamento e do material.

A Fig. 6.2 mostra a reação no engaste em função do tempo (linhas sólidas), para as três diferentes discretizações, com 0 modelo A de dano a gradiente $(\mathrm{q}=0.05 \mathrm{~N}$, $\mathrm{G}=0, \mathrm{a}=0$ ).

A linha pontilhada representa a solução elástica. 0 bloco de onda anda elasticamente até encontrar 0 engaste. Devido à reflexão, a tensão dobra $(\sigma=1.06 \bar{\sigma})$ e se inicia a danificação ao longo da barra. 0 exemplo mostra que há independência dos resultados na discretização.

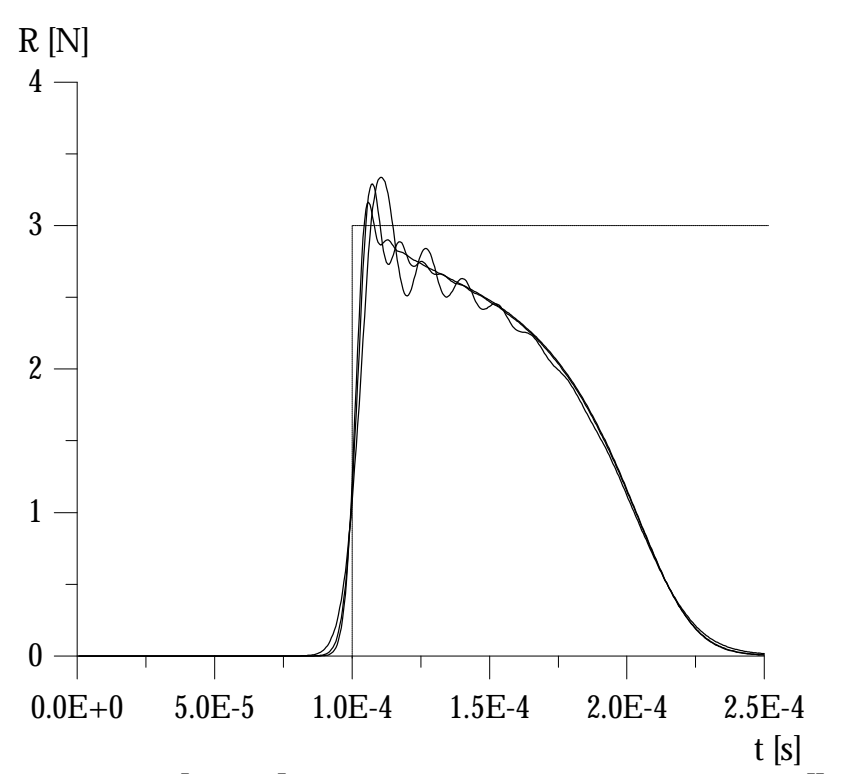

Figura 6.2. - Evolução da reação no engaste para as três malhas.

A curva da malha 3 da Figura 6.2 é individualizada (curva vermelha da Figura 6.3), e compara-se a evolução da reação no engaste com 0 a evolução do valor de dano ao longo do tempo. Percebe-se que a reação tende a zero para o valor de dano tendendo à unidade. 


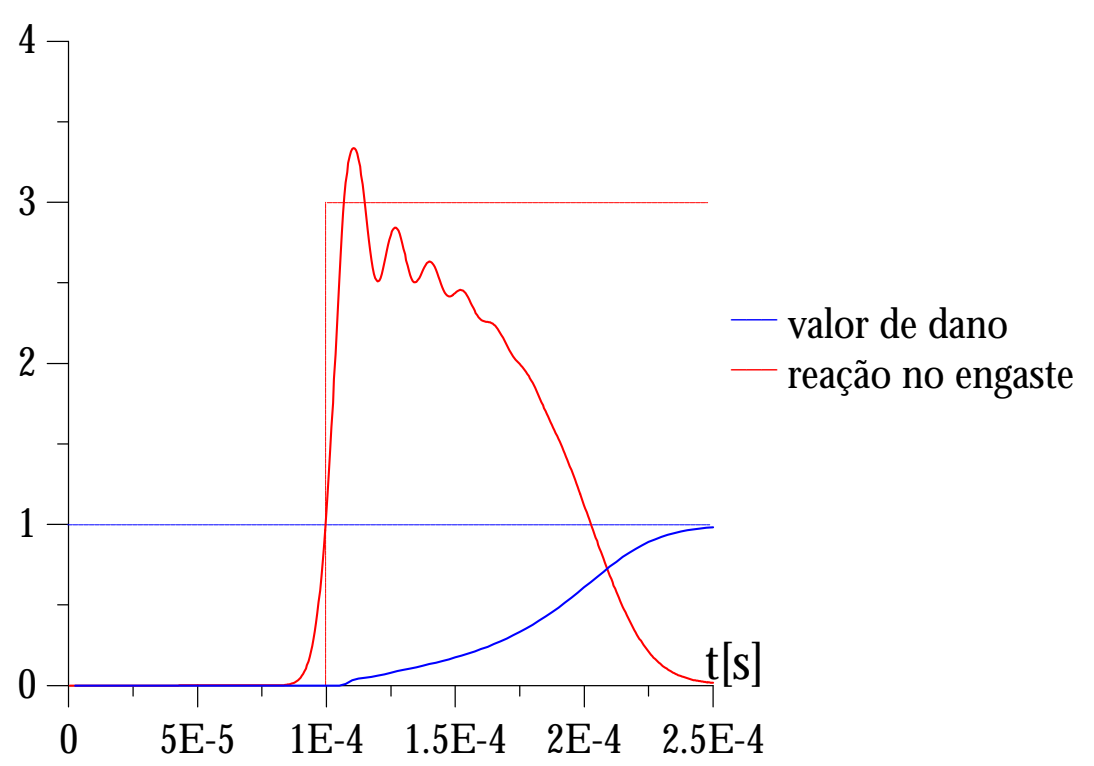

Figura 6.3. Curva R ou D x t para o modelo de dano não - local.

As Figuras 6.4a,b mostram o perfil de dano para três valores diferentes dos termos de difusão $\mathrm{G}_{1}$ e $\mathrm{G}_{2}$, respectivamente, mantendo constantes os demais parâmetros. Observa-se, na Figura 6.4a, que o gradiente do dano é nulo na extremidade do engaste, enquanto que na Figura $6.4 \mathrm{~b}$, percebe-se que o Laplaciano é nulo no engaste; mostrando coerência com as condições de contorno estabelecidas.

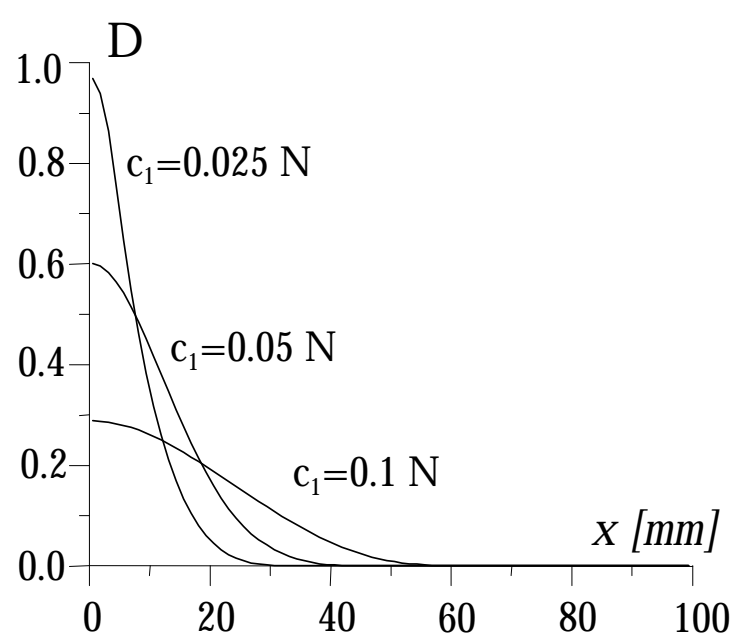

(a)

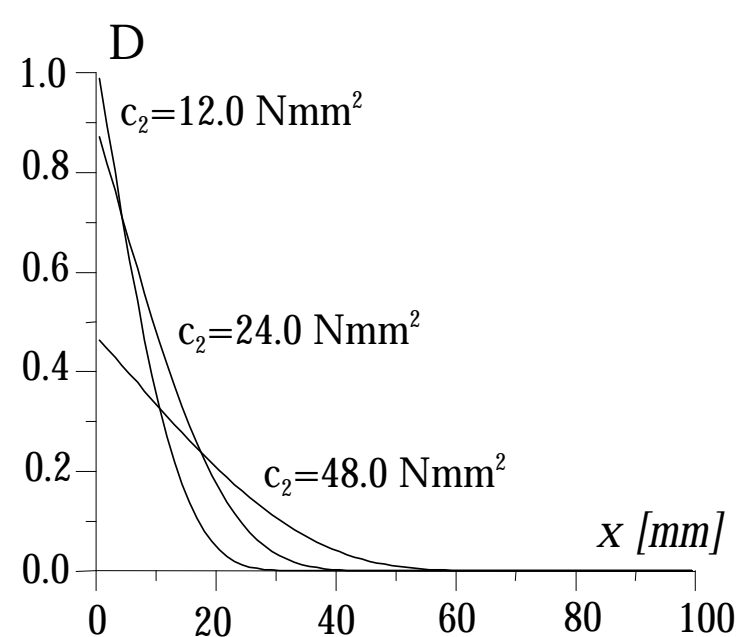

(b)

Figura 6.4. Perfil de dano em $\mathrm{t}=2 . \mathrm{x}^{4} 0^{4}$, para (a) modelo $\mathrm{A}$ e (b) modelo $\mathrm{C}$ de dano, para três valores diferentes dos termos de difusão.

A barra da Figura 6.1 é analisada para valores particulares dos parâmetros de difusão e da constante $\mathrm{a}: \mathrm{G}_{1}=0.05 \mathrm{~N}, \mathrm{C}_{2}=0, \mathrm{a}=0$ (modelo A, conforme Capítulo 
4), $\mathrm{G}_{1}=0.05 \mathrm{~N}, \mathrm{C}_{2}=0, \mathrm{a}=1$ (modelo $\mathrm{B}$ ) e $\mathrm{G}=0.05 \mathrm{~N}, \mathrm{C}_{2}=12 \mathrm{Nm}^{2}, \mathrm{a}=0$ (modelo C).

A Figura 6.5 apresenta a dissipação de energia para os três modelos. Percebe-se que a regularização, como era de se esperar, mantém a dissipação de energia constante para as análises feitas com diferentes malhas, diferentemente dos resultados obtidos com o modelo local.

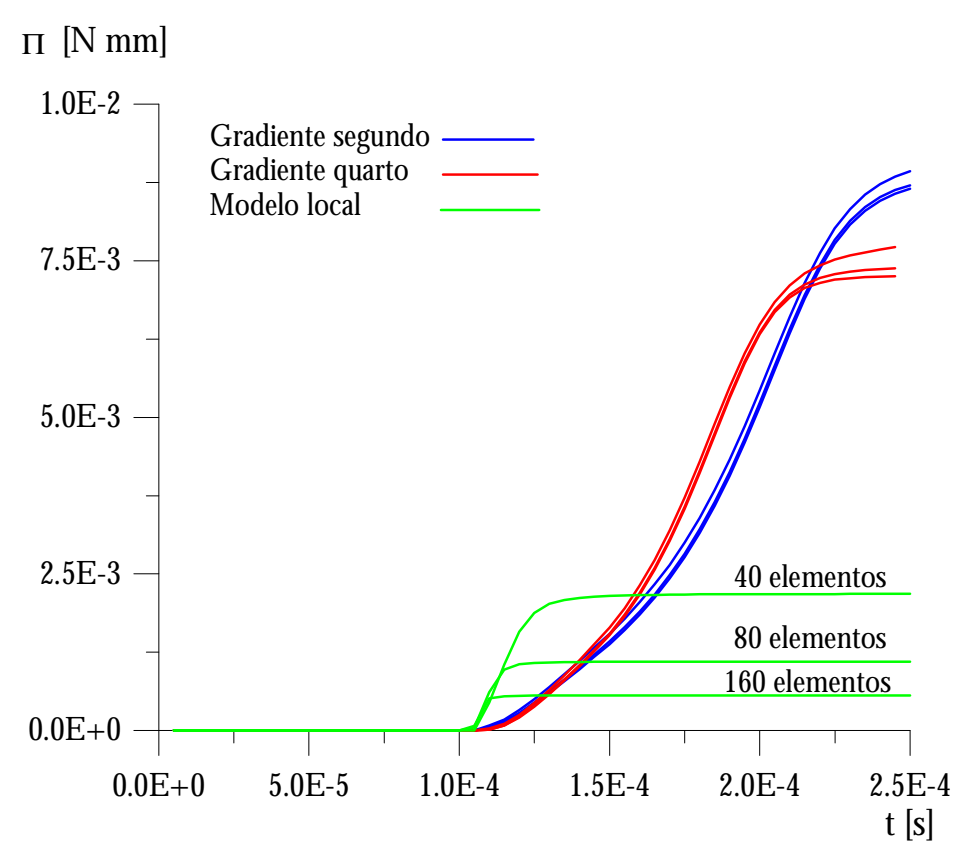

Figura 6.5. D issipação de energia no tempo para os modelos de dano local, A e B.

Os perfis de dano no final das análises, são mostrados na Figura 6.6a,b,c, respectivamente. Eles convergem rapidamente para um perfil independente da malha, com o refinamento. A evolução do dano durante a análise, para a malha 2, é mostrada nas Figuras 6.6d,e,f.

A evolução do dano durante as análises dos modelos A e B, em alguns elementos da malha 3, é mostrada nas Figuras 6.7a,b, respectivamente (elemento 1 é 0 elemento mais próximo ao engaste e 0 elemento 60 é o último elemento que se danifica durante a análise).

No início do processo inelástico, de $\mathrm{t}=10^{-4}$ e $\mathrm{t}=1.4 \times 10^{-4} \mathrm{~s}$, a zona danificada aumenta e um número cada vez maior de elementos entra em regime de danificação; depois forma-se a banda de localização e sua largura diminui progressivamente; os elementos distantes do engaste descarregam e o dano estaciona para um número cada vez maior de elementos. Percebe-se que, para 0 modelo $B$, este fenômeno é menos pronunciado. 


\section{Lanissa Driemier}
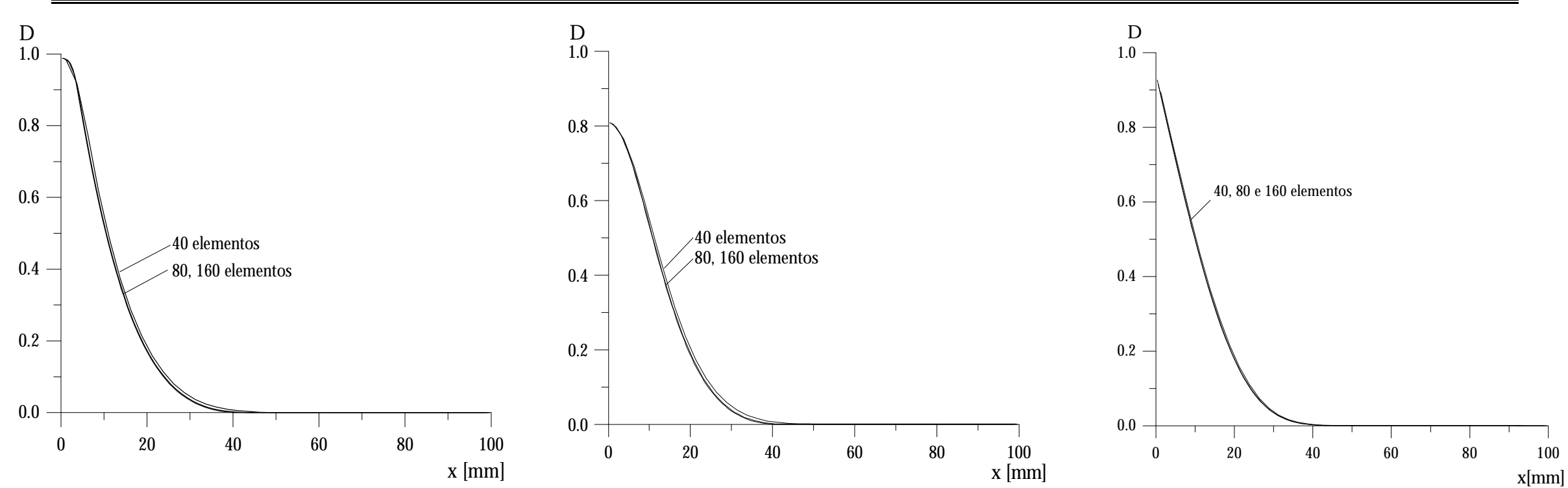

(a)

(b)

(c)

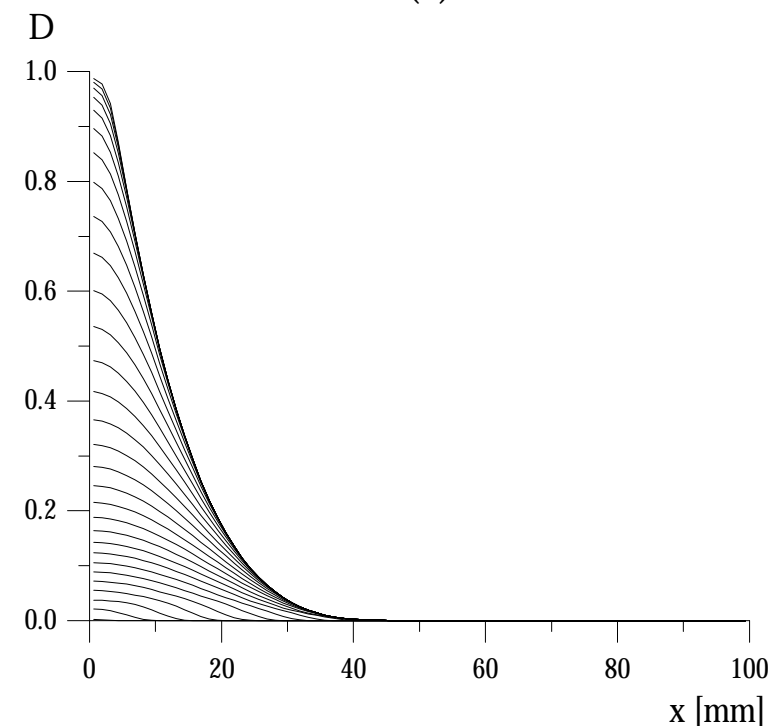

(d)

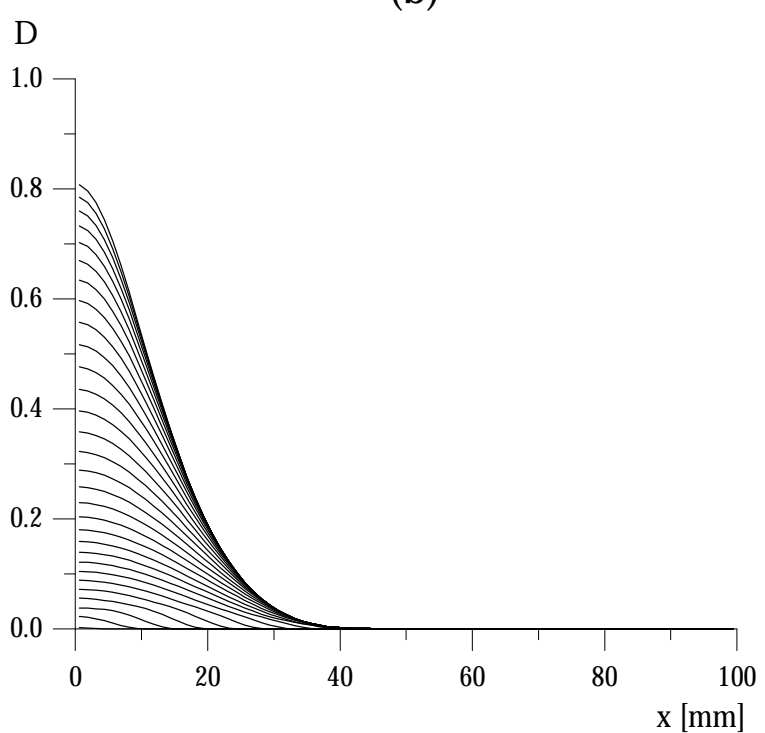

(e)

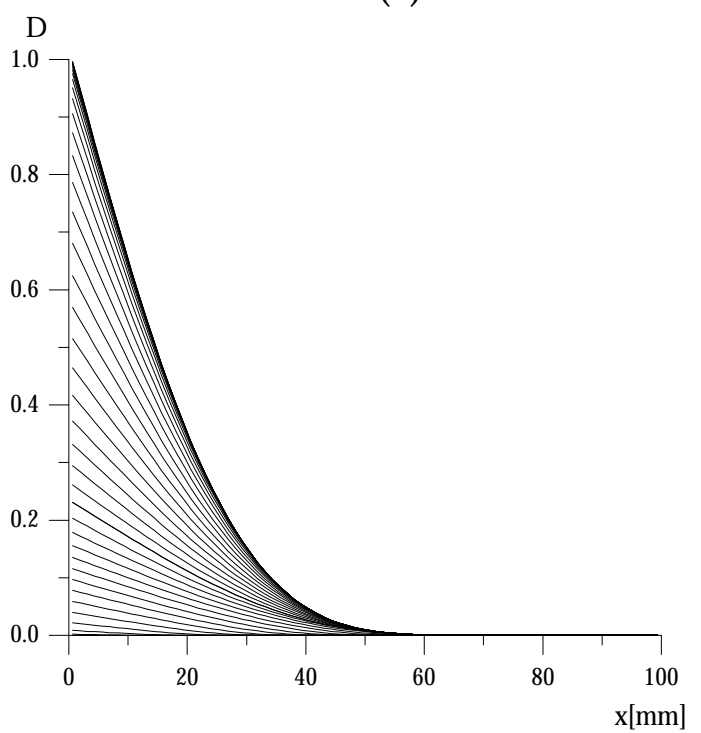

(f)

Figura 6.6. Dano no final das análises e a evolução do dano para os modelos A(a,d), B(b,e) e C(c,f), respectivamente. 


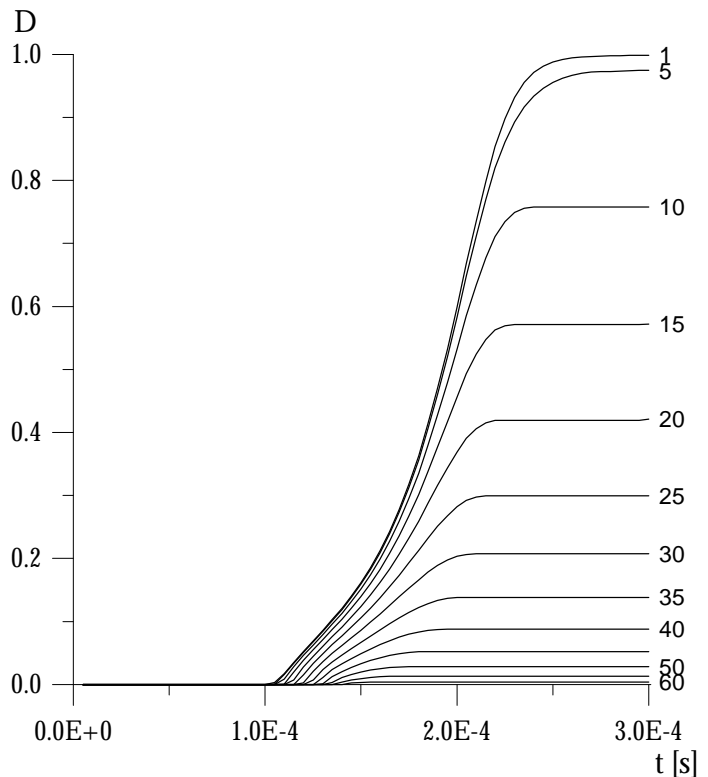

(a)

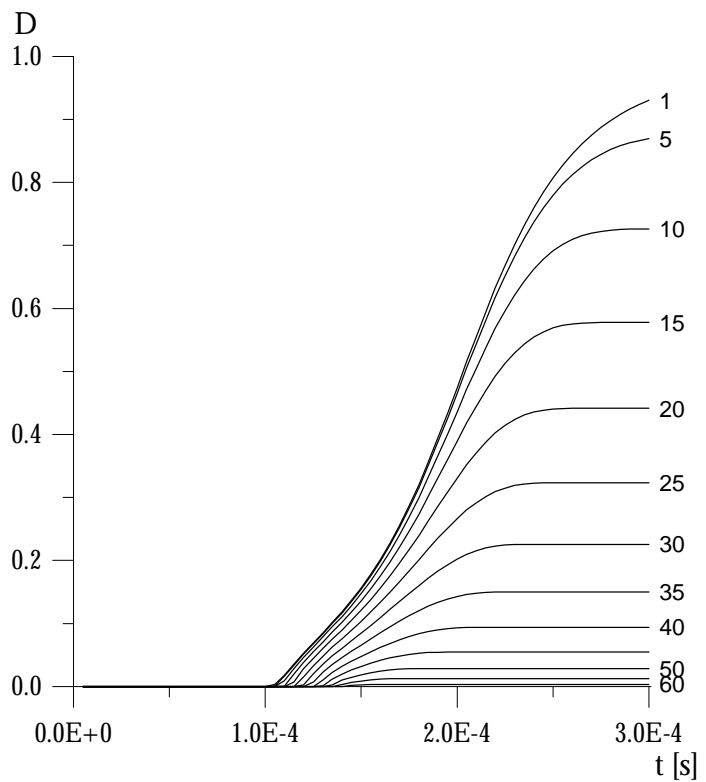

(b)

Figura 6.7. (a) Evolução do dano para modelo A. (b) Evolução do dano para modelo B.

\subsection{Exemplos bidimensionais}

\subsubsection{Exemplo dinâmico}

A Figura 6.8 mostra as características geométricas e de carregamento. 0 mesmo exemplo foi analisado em plasticidade com gradiente por Slyus $^{8}$, Pamin' $^{4}$, e no contexto da mecânica do dano por $\mathrm{Cam}^{10}{ }^{10}$ Comi \& Driemeie ${ }^{11}$.

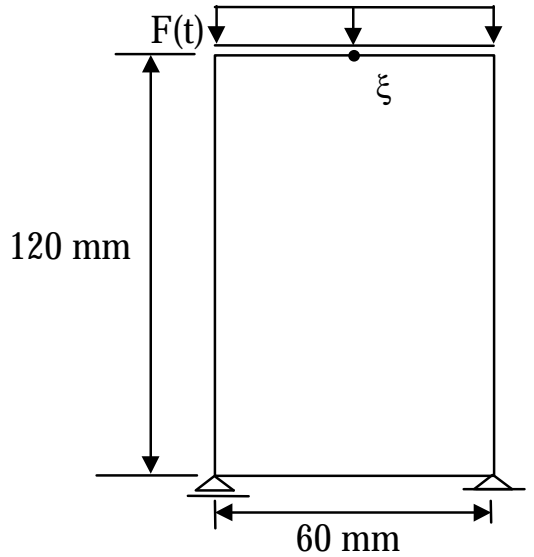

(a)

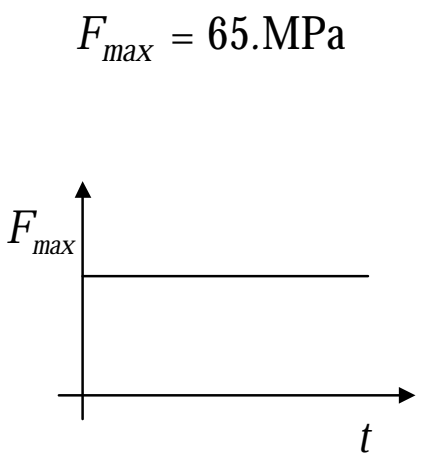

(b)
$\mathrm{G}=4000 . \mathrm{MPa}$

$v=0.49$

$\mathrm{b}_{1}=\mathrm{b}_{2}=0$.

$\mathrm{b}_{3}=0.25 \mathrm{MPa}$

$\mathrm{b}_{4}=0.4 \mathrm{MPa}$

$\rho=5.10^{-9} \frac{\mathrm{Ns}^{2}}{\mathrm{~mm}^{4}}$

(c)

Figura 6.8. (a) Caractenísticas geométricas, (b) dados do carregamento e (c) caractenísticas do material. 
0 problema, foi analisado à deformação plana primeiramente com o modelo local $\left(\mathrm{G}_{1}=\mathrm{C}_{2}=0\right)$. A onda de compressão se propaga elasticamente até atingir a base do espécime, quando a reflexão produz dano que se localiza para formar a banda de cisalhamento.

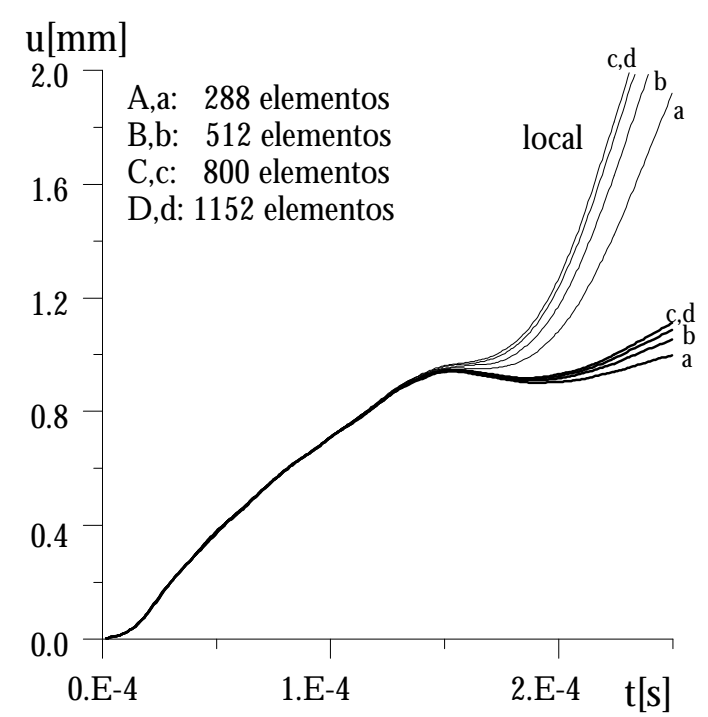

(a)

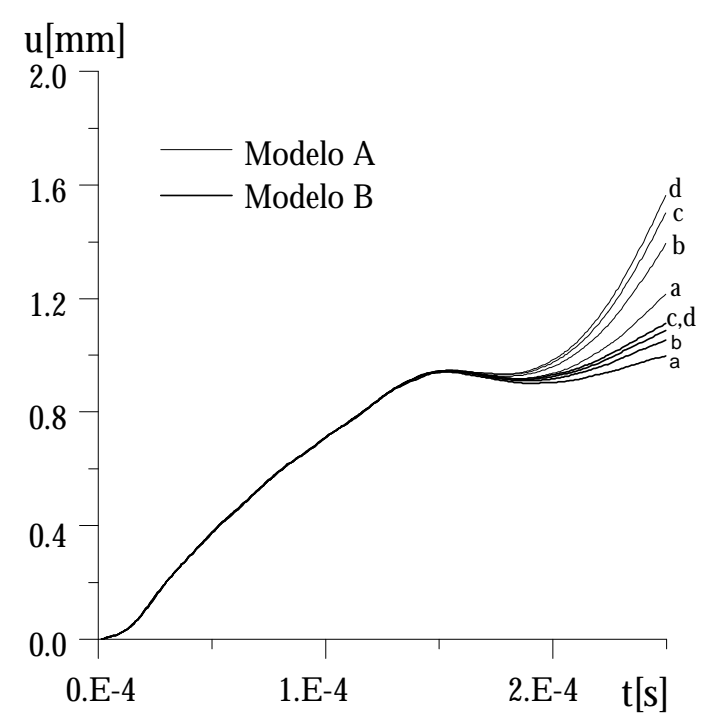

(b)

Figura 6.9. Deslocamento no tempo para modelos (a) de dano local e modelo B e (b) modelos B e A.

A Figura 6.9 mostra o deslocamento do ponto $\xi$ para as diferentes malhas: depois da localização a resposta se torna fortemente dependente da discretização. $\mathrm{Na}$ mesma figura é mostrado o resultado obtido para o modelo de dano $\mathrm{B}$, com parâmetro de difusão variável. Neste caso, depois da localização os resultados permanecem praticamente independentes da malha, até que o dano se aproxima do valor crítico um na zona de localização. Neste estágio, novamente se observa a dependência dos resultados na discretização espacial. Isto ocorre devido ao fato que o comprimento característico do modelo de dano à gradiente, implicitamente introduzido pelo parâmetro de difusão $\frac{\mathrm{G}}{1-\mathrm{D}} \nabla^{2} \mathrm{D}$, diminui com o valor do dano; portanto malhas cada vez mais refinadas são necessárias para representar corretamente o fenômeno com o aumento do dano ('meshrefinement').

Como solução alternativa, sugere-se a apropriada substituição do modelo contínuo para um descontínuo, onde é permitida descontinuidade de deslocamento. 0 fenômeno da dependência de malha é muito mais pronunciado com um parâmetro de difusão constante, como mostra a Fig. 6.9b. 
(a)
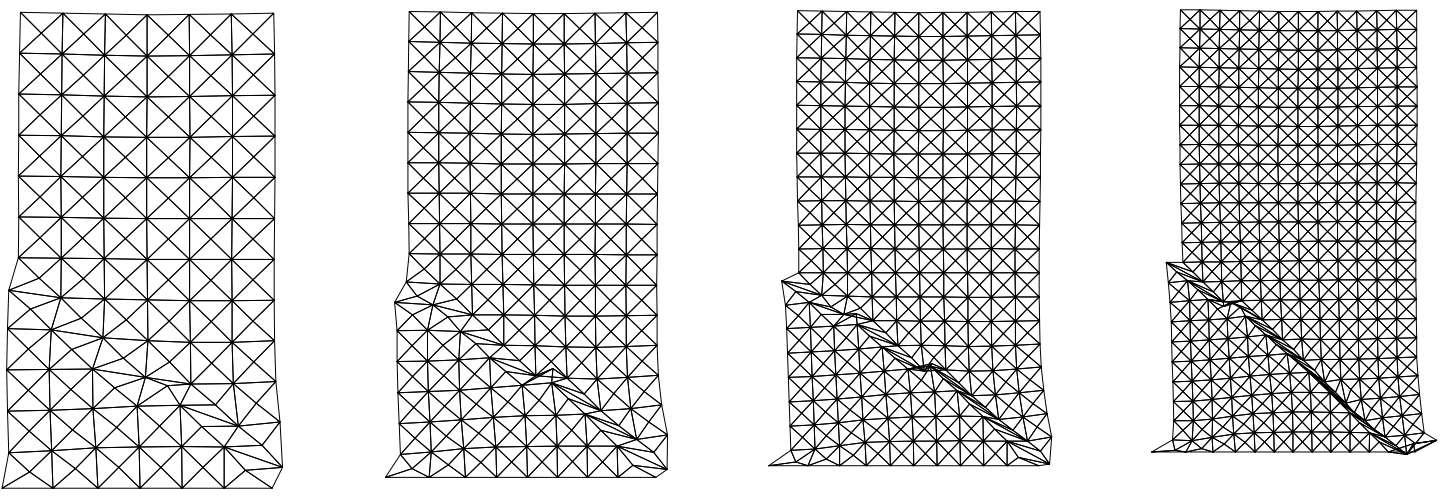

(b)
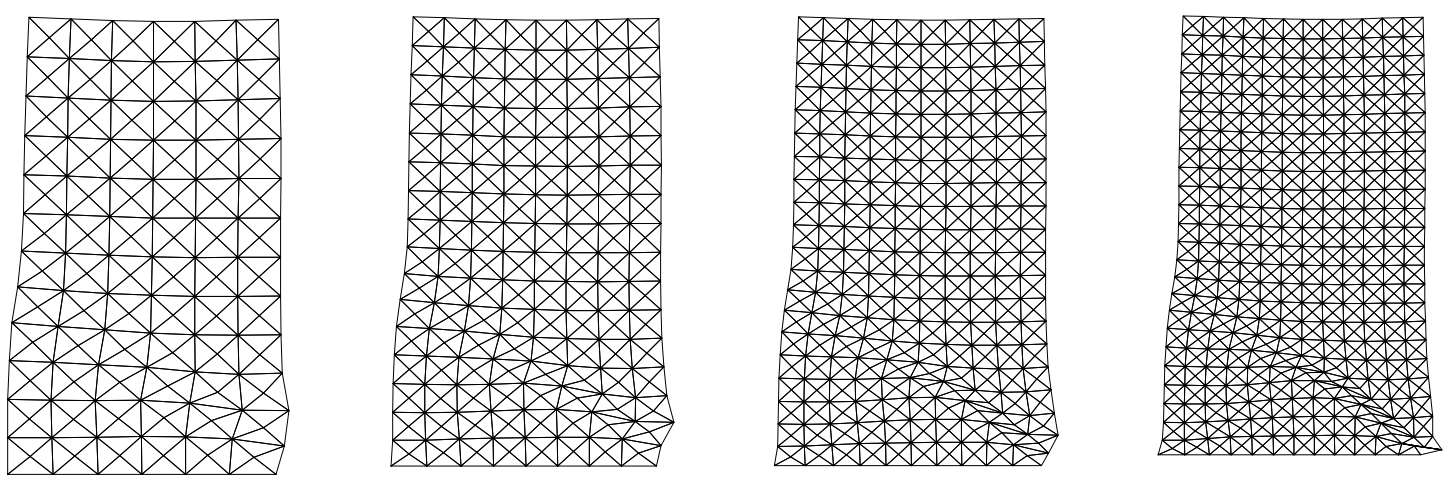

(c)
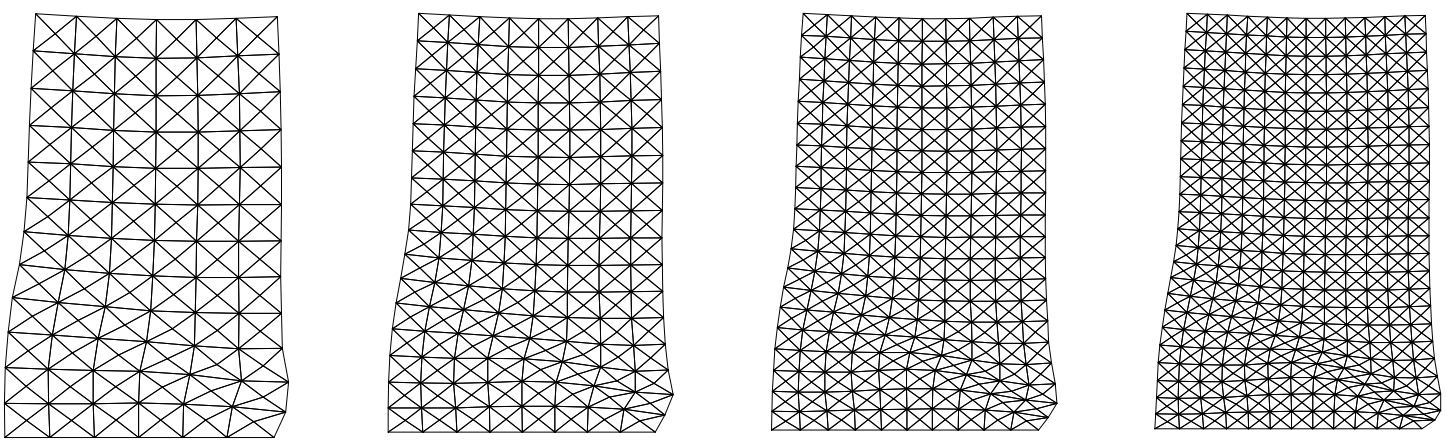

Figura 6.10. Configuração deformada para: (a) modelo local, (b) modelo A, (c) modelo B.

As Figuras 6.10a,b,c mostram o espécime deformado para quatro diferentes malhas, quando a análise atinge $\mathrm{t}=1.65 \times 10^{-4} \mathrm{~s}$ : (a) modelo local, (b) modelo $\mathrm{A}$ (G $=30 . N, a=0)$, (c) modelo $B(q=30 . N, a=1)$. A Figura mostra $a$ independência na discretização para os modelos não - locais. D evido à maior difusão do dano para 0 caso do modelo B, a resposta perto do nível crítico (fraturamento) é menos localizada. 


\subsubsection{Viga parede com entalhe}

0 teste experimental de Mazars ${ }^{63}$ em um espécime de concreto é simulado numericamente (Figura 6.11a). O mesmo exemplo foi tratado por Frémond \& Nedjar ${ }^{9}$ usando um modelo de dano com gradiente em taxas.

O s dados experimentais disponíveis do material são o módulo elástico e resistência à tração e compressão, que não são suficientes para identificar os parâmetros do modelo de dano: $b_{i}$ e c Portanto, foi escolhido $b_{2}=0$, de modo que se obtém um Problema de Complementariedade Linear para a fase de correção e os outros parâmetros $\left(\mathrm{b}_{1}, \mathrm{~b}_{3}, \mathrm{k}\right)$ foram manipulados de modo a melhor adaptar os resultados numéricos àqueles experimentais em termos de força de abertura $\mathrm{F}$. Os parâmetros obtidos para 0 modelo são: $E=30,000 \mathrm{MPa}, v=0.2, \quad b_{1}=14.946 \mathrm{MPa}$, $\mathrm{b}_{3}=0.0028 \mathrm{MPa}, \mathrm{k}=0.00016 \mathrm{MPa}, \mathrm{G}=9 . \mathrm{N}$. Metade do espécime foi discretizado em duas malhas de elementos finitos triangulares a deformação constante (conforme elemento CST-3 do capítulo anterior), Figuras 6.11b,c.

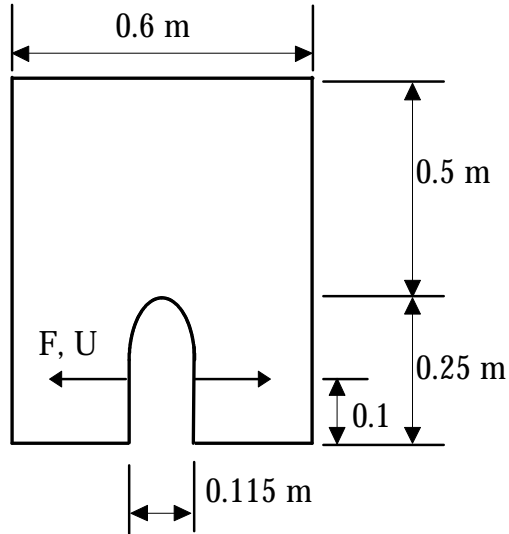

largura: $0.12 \mathrm{~m}$

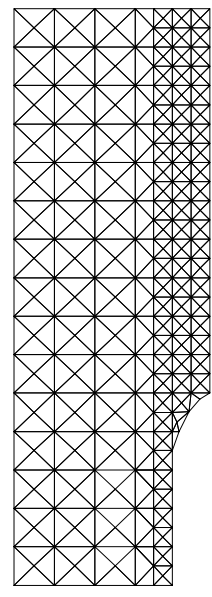

(b)

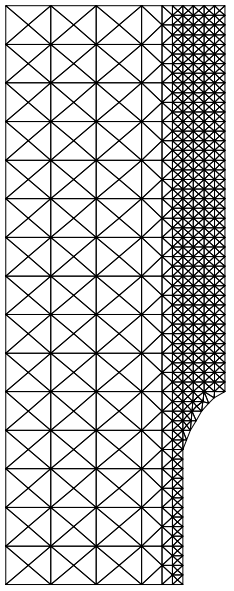

(c)

(a)

Figura 6.11. (a) Dados geométricos do espécime; Malhas com (b) 516 e (c) 1230 elementos.

Força de abertura F versus abertura do nó 2U estão plotados na Figura 6.12, juntamente com os resultados experimentais obtidos a partir das referências Mazars ${ }^{63}$ e Frémond \& Neejar ${ }^{9}$. Além do comportamento análogo entre resultados numéricos e experimentais, os resultados das duas discretizações são similares.

As evoluções das deformações são mostradas nas Figuras 6.13 e 6.14, com deslocamentos aumentados 500 vezes. As Figuras 6.13 e 6.14 correspondem às malhas de 516 e 1230 elementos. Ao final da análise, com o surgimento da fratura (dano atinge valores próximos à unidade), as figuras mostram a tendência da deformação concentrar-se novamente em uma única coluna vertical de elementos. 


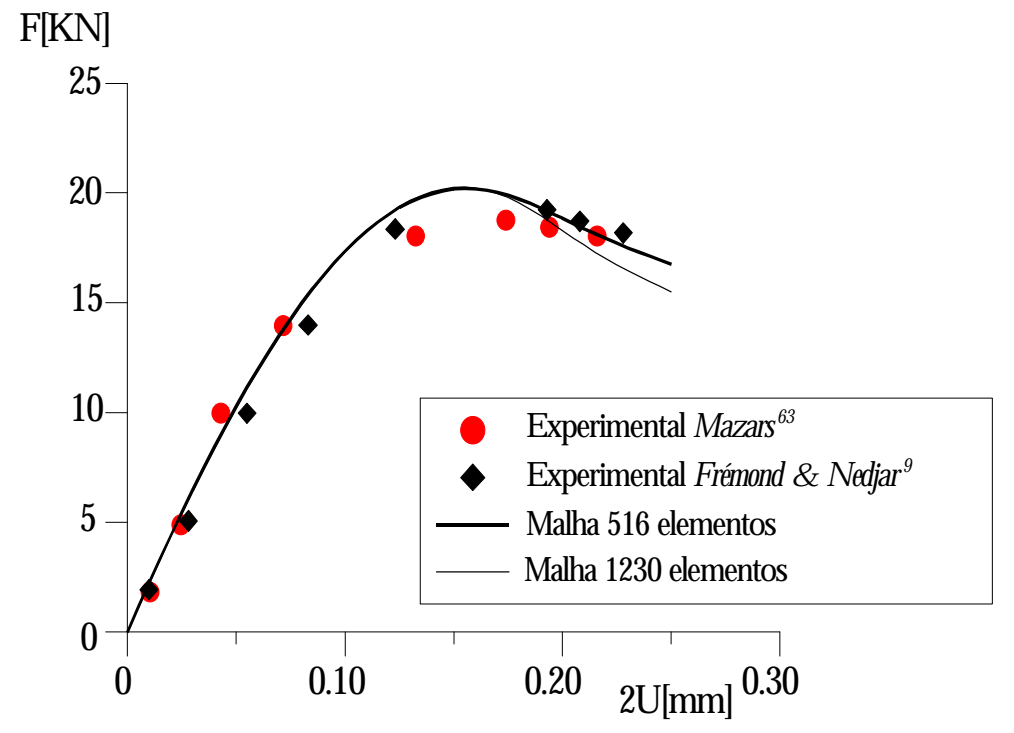

Figura 6.12. Fonça aplicada versus abertura do entalhe.

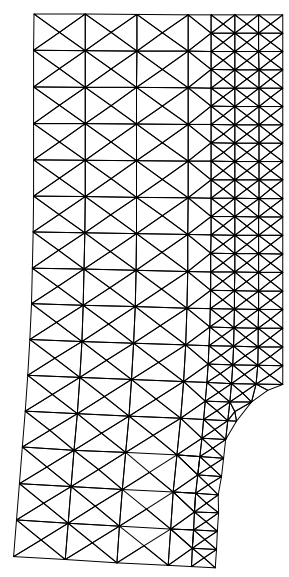

(a)

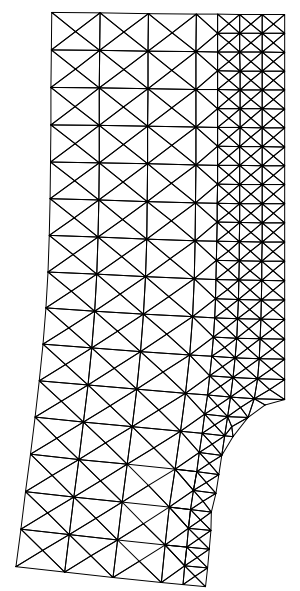

(b)

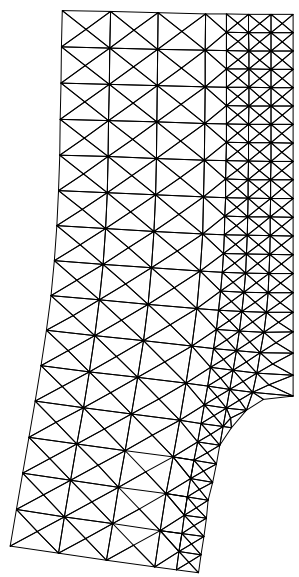

(c)

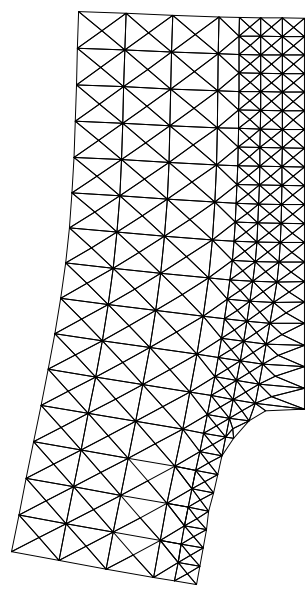

(d)

Figura 6.13. Evolução da deformação, malha de 516 elementos e aberturas de (a)0.1, (b)0.18, (c)0.26 e (d) $0.34 \mathrm{~mm}$.

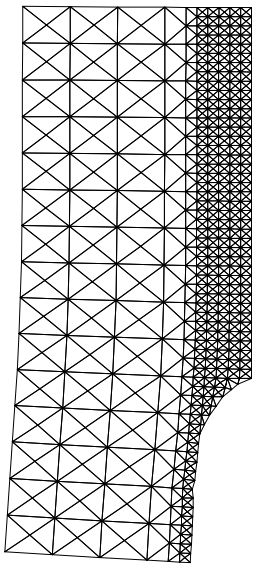

(a)

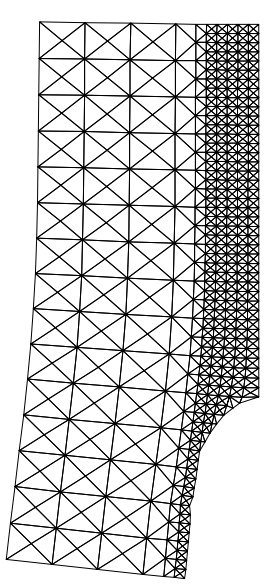

(b)

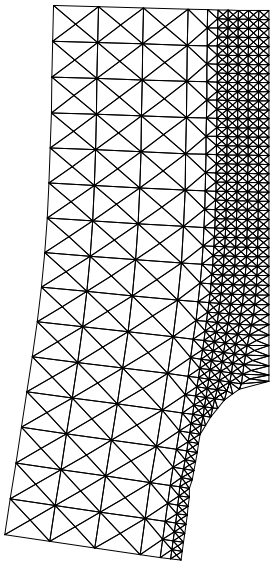

(c)

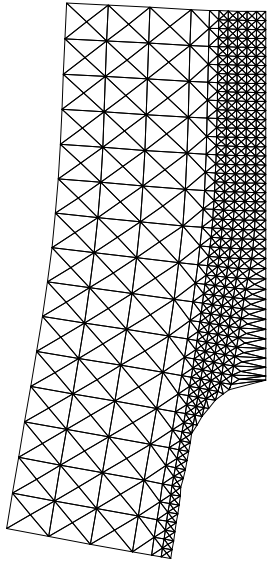

(d)

Figura 6.14. Evolução da deformação para malha de 1230 elementos e aberturas: (a)0.1,(b)0.18,(c)0.26 e (d) $0.34 \mathrm{~mm}$. 


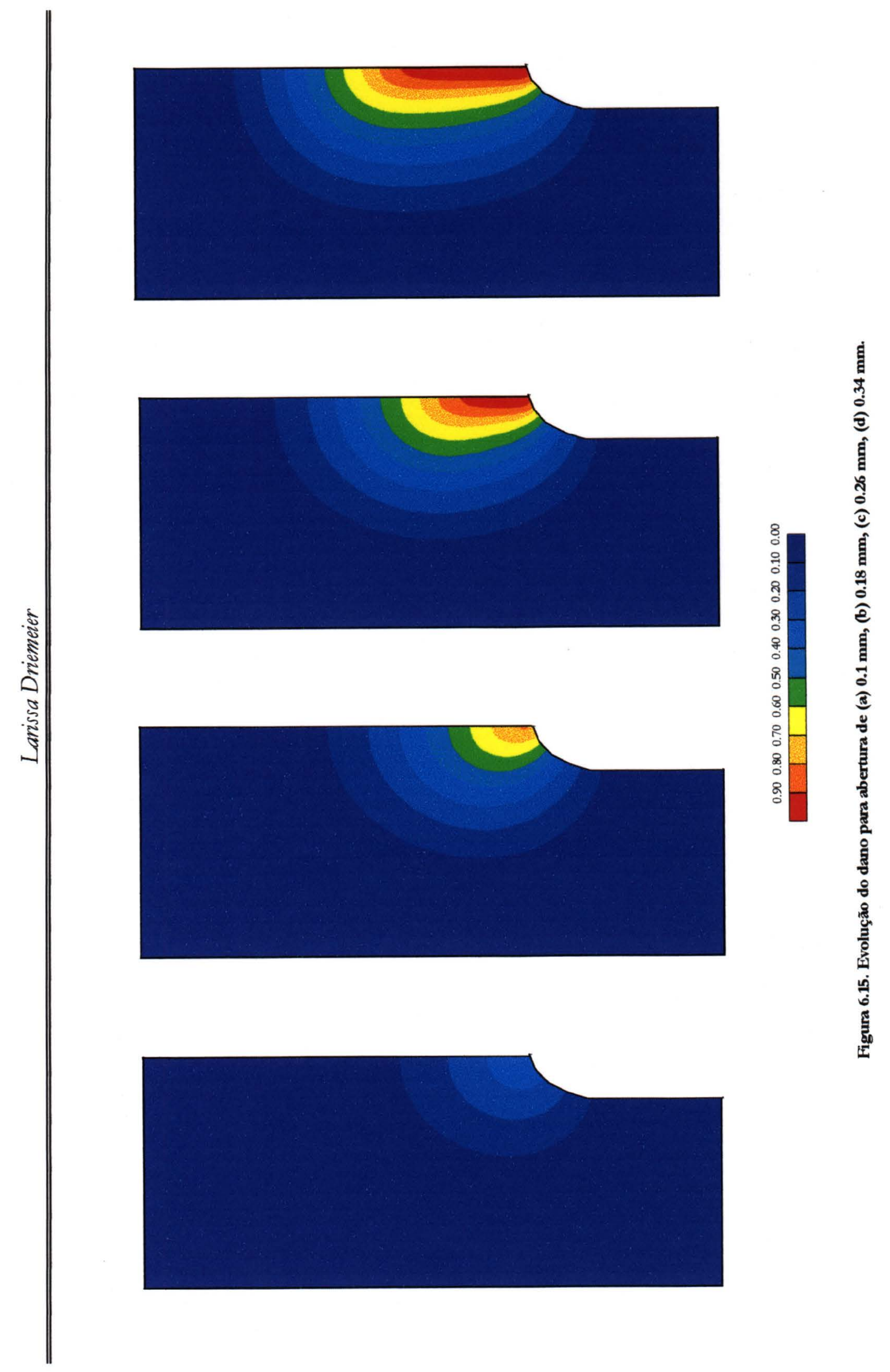


A evolução da zona com dano é mostrada na Figura 6.15. AS Figuras 6.15a,b,c,d correspondem às aberturas de 0.1, 0.18, 0.26 e $0.34 \mathrm{~mm}$, respectivamente para as mesmas dimensões de abertura das figuras anteriores. Observa-se que, devido ao termo gradiente, 0 dano inicialmente se difunde (conforme Figuras 6.15a,b), porém, com a evolução da análise (Figuras $6.15 \mathrm{c}$,d) a largura da zona de dano se mantém constante. $\mathrm{O}$ valor do dano cresce no centro, em uma estreita faixa vertical, simulando corretamente a fissuração do espécime.

\subsubsection{Viga com entalhe}

0 terceiro exemplo bidimensional refere-se a um teste em uma viga com entalhe de concreto, analisada por Meger $\&$ al. ${ }^{64}$ assumindo uma aproximação de fissuração contínua (smered cadk approad). Os testes experimentais deste exemplo também foram realizados por Mejer $\notin$ al. ${ }^{64}$ com controle de deslocamentos. A Figura 6.16 mostra os dados geométricos da viga e os parâmetros do modelo A de dano, obtidos de maneira a melhor adaptar a curva numérica de tração uniaxial do concreto àquela experimental fornecida em Mejer $\notin a$ al. $^{64}$. A análise foi feita usando três malhas A, B e C de 724, 1412 e 2148 elementos CST-3 (Figura 6. 17).

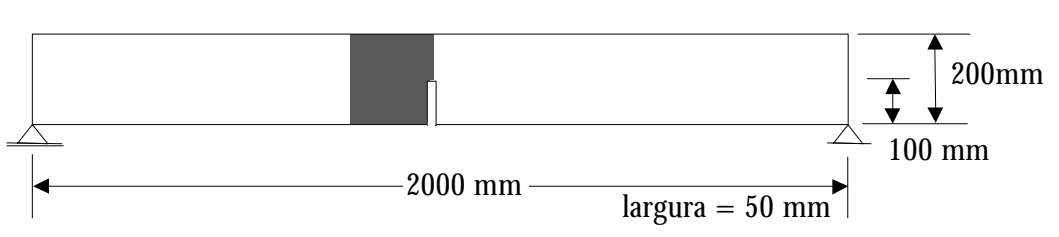

$$
\begin{aligned}
& \mathrm{E}=30000 \mathrm{~N} / \mathrm{mm}^{2} \\
& \mathrm{~b}=32.0 \mathrm{~N} / \mathrm{mm}^{2} \\
& \mathrm{~b}_{2}=0 \\
& \mathrm{~b}_{3}=0.0004 \mathrm{~N} / \mathrm{mm}^{2} \\
& \mathrm{k}=0.001 \mathrm{~N} / \mathrm{mm}^{2} \\
& \mathrm{G}=9.0 \mathrm{~N}
\end{aligned}
$$

Figura 6.16. Geometria da viga e parâmetros do material.

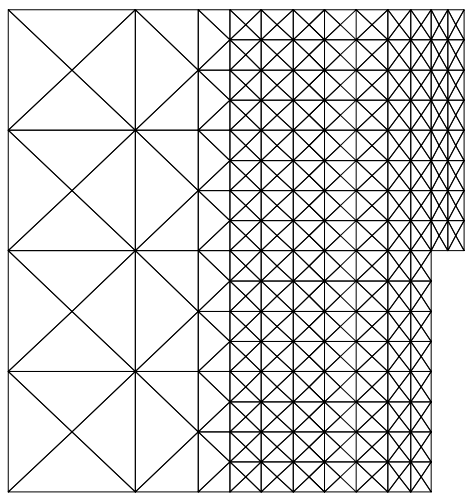

(a)

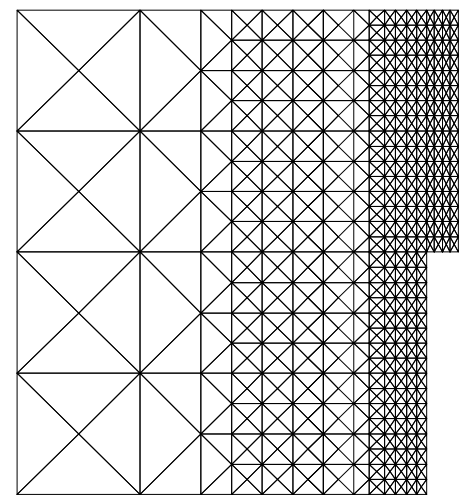

(b)

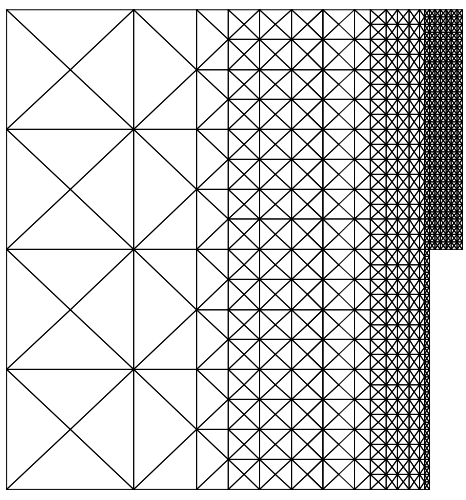

(c)

Figura 6. 17. Malhas usadas na análise da estrutura: (a) 724 (malha 1), (b) 1412 (malha2) e (c) 2148 (malha 3) elementos.

Na Figura 6.18, mostra-se as respostas Força - D eflexão. Pode-se observar uma boa aproximação entre resultados numéricos e experimentais. Além disso, as curvas computadas convergem para uma solução independente da malha, com 0 refinamento, também no ramo descendente. 


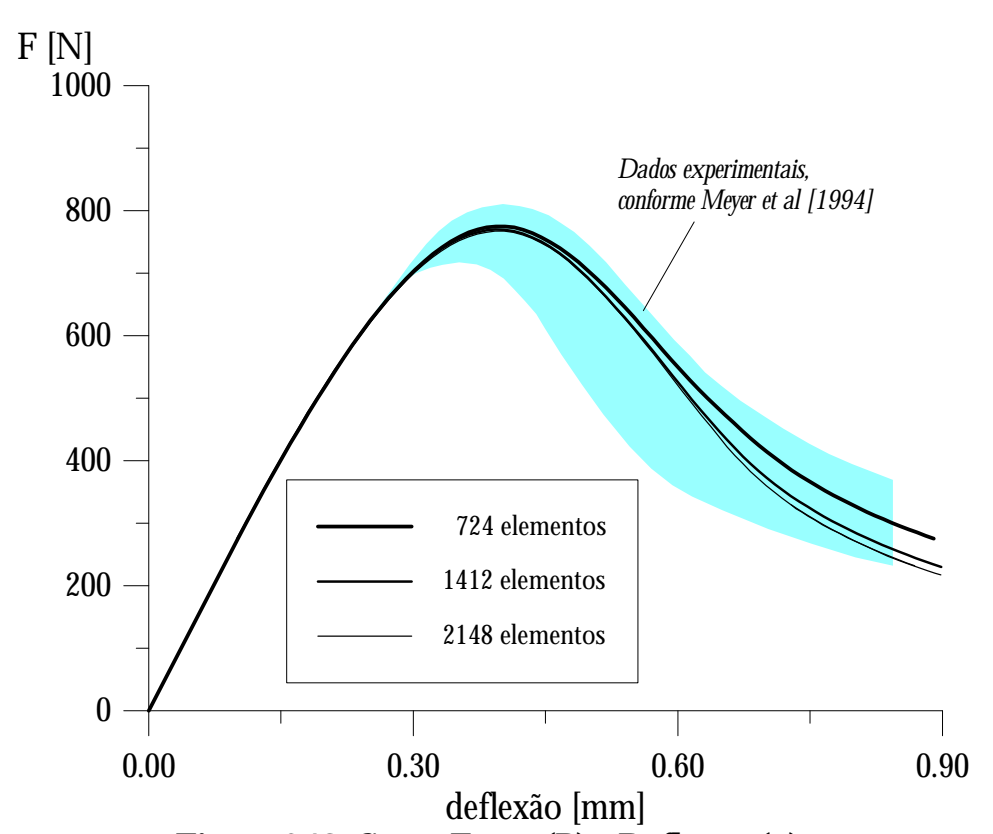

Figura 6.18. Curva Fonça (P) - Deflexão (u).

A Figura 6.19 compara as configurações deformadas, para uma deflexão de 0.5 $\mathrm{mm}$, obtida com as três malhas (fator de ampliação 300). D evido à localização do dano, altas deformações podem ser observadas perto do entalhe, mas a configuração de deformação é praticamente independente da malha.

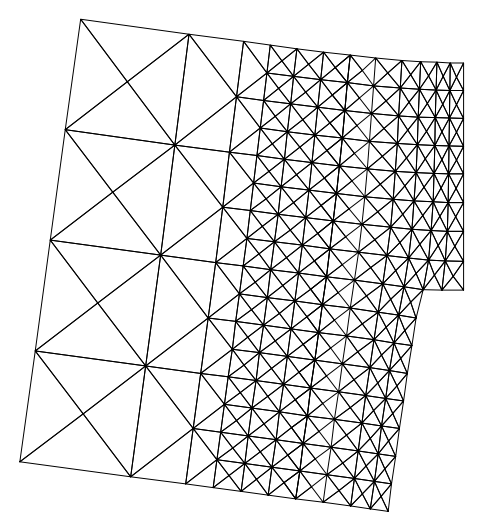

(a)

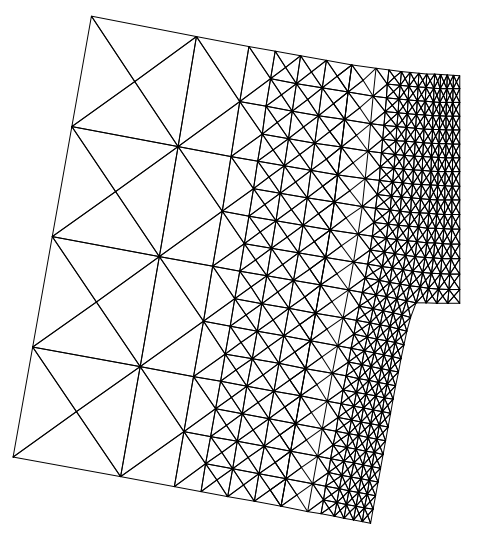

(b)

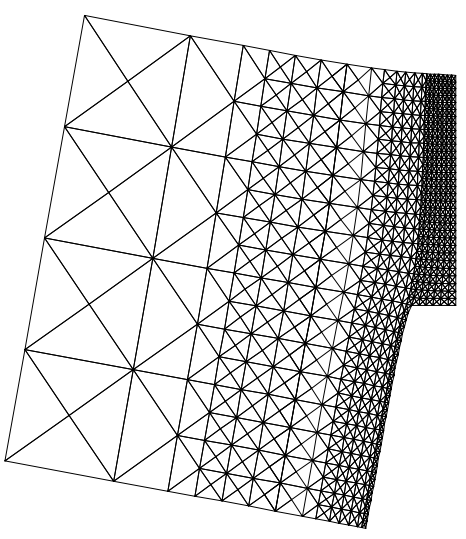

(c)

Figura 6.19. Configuração da deformada com deslocamento central imposto de $0.5 \mathrm{~mm}$, para as malhas (a) 1 , (b) 2 e (c) 3.

A Figura 6.20 mostra as superfícies de dano da região sombreada da Figura 6.16 para a malha 2 à deflexão imposta de $u=0.3 \mathrm{~mm}, \mathrm{u}=0.7 \mathrm{~mm}, \mathrm{u}=0.9 \mathrm{~mm}$ e $\mathrm{u}=1.1 \mathrm{~mm}$. $\mathrm{O}$ dano inicialmente se desenvolve ao redor da ponta do entalhe e depois cresce em uma faixa vertical, permitindo a simulação da propagação de uma macrofissura, levando ao colapso. 

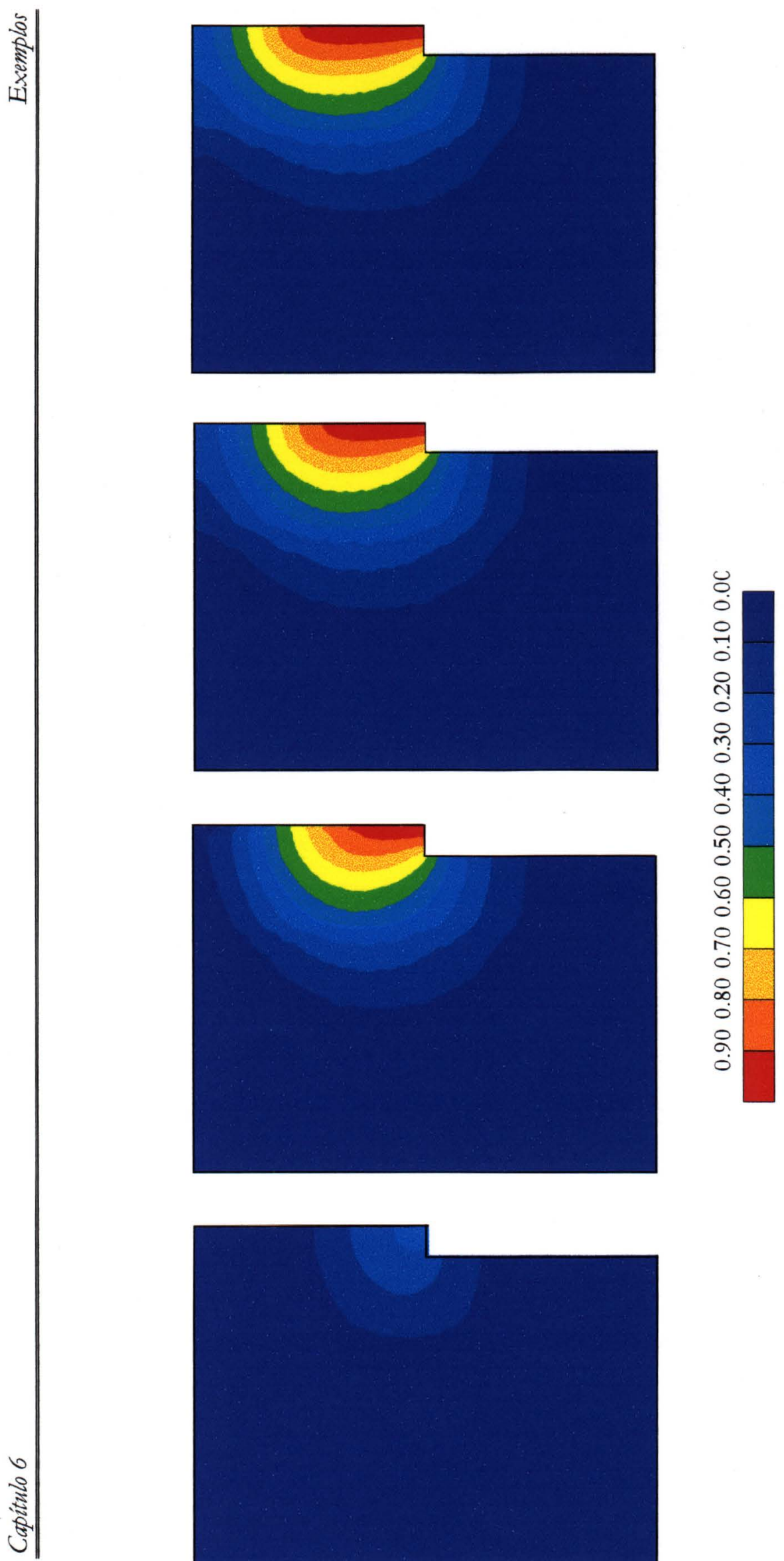

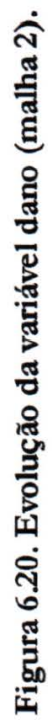

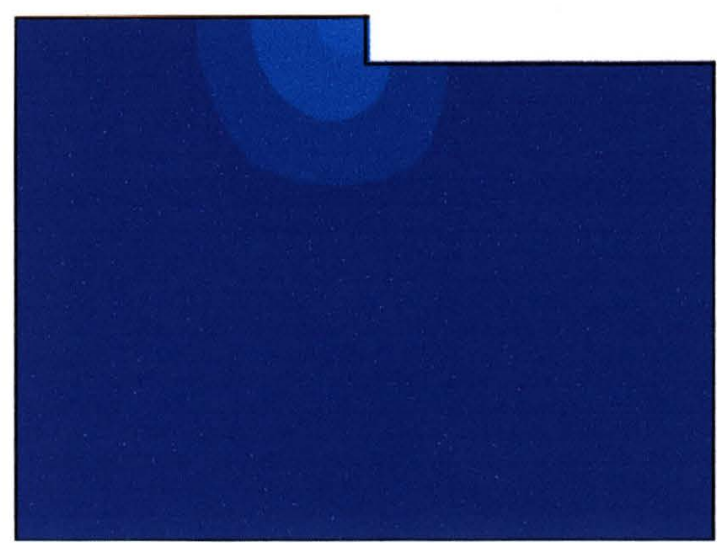




\section{Síntese do Conteúdo e Conclusões}

O trabalho enfoca o problema de localização de deformações decorrente do emprego de modelos constitutivos com sattering

O segundo capítulo apresenta o fenômeno da localização que, em uma análise numérica, explica o surgimento de problemas como a dependência dos resultados com a discretização adotada. Em outras palavras, nos modelos constitutivos clássicos, baseados em uma descrição local do comportamento do material, a largura da zona de localização tende a zero, uma vez que a simulação procura encontrar uma condição compatível com uma fratura discreta.

Entre as técnicas de regularização da resposta numérica propostas na literatura, 0 trabalho trata daquela que consiste na inclusão de gradientes de ordem superior na função de escoamento. Os parâmetros que acompanham os termos com gradiente são chamados parâmetros de difusão e são considerados neste trabalho, como característica do material.

No capítulo 3, através de exemplos simples com modelos elasto-plásticos, apresenta-se uma análise do efeito dos gradientes de segunda e quarta ordem. A analogia com 0 estudo do surgimento de ondas estacionárias permite interpretar de maneira mais clara a questão da largura finita da banda de localização que surge com a regularização do modelo.

Segue o estudo de regularização dos modelos elásticos com dano. Diferentemente do modelo elasto-plástico, regularizado com gradientes segundo e quarto e parâmetros de difusão constantes, não se apresenta, no modelo de dano, uma largura de banda fixa e finita, sendo que a mesma tende a zero na medida que 0 
valor do parâmetro de dano tende à unidade. Três modelos com gradiente foram estudados: gradiente segundo com parâmetro de difusão constante, gradiente segundo com parâmetro de difusão não constante, gradientes segundo e gradiente quarto com parâmetros de difusão constantes. Novamente a questão da largura da banda de localização foi abordada via uma análise de propagação de ondas. Conclui-se que é possível reproduzir uma largura finita ao final da análise, no caso de parâmetros de difusão variáveis com o dano; 0 gradiente quarto tende a retardar o processo de formação da descontinuidade, ou largura nula.

Os modelos apresentados foram implementados com o emprego do Método dos Elementos Finitos em sua formulação em variáveis generalizadas. A base variacional de tal técnica permite uma implementação numérica do modelo a gradiente sem a necessidade de impor a prioi condições extra de contorno. Os aspectos relacionados à formulação variacional e algoritmos de integração em passo finito dos modelos em estudo estão detalhados no Capítulo 5 desta tese.

Os exemplos ilustram os diversos aspectos do modelo, evidenciados nas análises teóricas realizadas no Capítulo 4, e apontam limites de aplicabilidade da técnica de regularização adotada. 
BIBLIOGRAFIA

\subsection{Referências bibliográficas}

[1] Rice, J. R. The localization of plastic deformations, In: Theoreical and Applied mehanics, Koiter, W. T. (ed.), North-Holland Publ. Comp., p.207-220 (1976).

[2] MaIER, G.; HUECKEL, T. Nonassociated and coupled flow nules of dastoplastiaty for rodk-likematerials, Int. J. Rock Mech. Min. Sci. \& G eomech. Abstr., 16, 77-92 (1979).

[3] Benallal, A.; Billardon, R.; Geymonat, G. Samemathematical aspets of the damage sutteningprddem In Cracking and damage, J. Mazars; Z.P. Bazant (eds.), Elsevier, Amsterdam, 247-258 (1988).

[4] RIzZI, E. Sulla localizzazione dale defomazioni in materiali e strutture, Tese de D outorado, Politecnico di Milano, Itália (1995).

[5] Bazant, Z.P.; Belytschko, T.; Chang, T.-P. Continuum therry for strains sattering ASCE J. Eng. Mech., 110, 1666-1692 (1984).

[6] Pijaudier-CabOt, G.; BAž ANT, Z.L. Nonlocal damage therry, ASCE J. Eng. Mech., 113, 1512-1533 (1987).

[7] MÜhlHaUs, B.; Aifantis, E.C. A variational piniplefor gradient plastiaty, Int. J. Solids Struct., 28, 845-857 (1991).

[8] SLUYS, L.J. Wave propagation, localization and dispersion in softering sodids Tese de D outorado, D elft University of Technology, Holanda (1992).

[9] FRÉMO ND , M.; NEDJAR, B. Damage, gadient of damageand priniple of virtual power, Int. J. Solids Structures, 33(8), 1083-1103 (1996).

[10] Co MI, C. Computational moddling of gradient-enhancel damagein quasi-brittlematerials Mech. Cohes. - Frict. Mater., 4(1), 17-36 (1999). 
[11]COMI, C.; D RIEMEIER, L. On gradient regularization for numerical analyses in the presence of damage, In: Mateial Instabilities in Solids de Borst, R.; van der Giessen, E. (eds), capítulo XVI, p.425-440, (1998).

[12] BILLARD O N, R. Etude de la nupture par la mecanique de l'endommagement, Tese de D outorado, Paris 6, França, (1989).

[13] SluYS, L.J.; DE BORST, R.; MÜHLHAUS, H.B. Wave propagation, localization and dispession in a gadient dependent meelum Int. J. Solids Struct., 30, 1153-1171 (1993).

[14] PAMIN, J. Gradient-dependent plastiaityin numerical similation of localization phenomena, Tese de D outorado, D elft University of Technology, Holanda (1994).

[15]Comi, C.; PEREgO, U. A generalized variable formulation for gradient-dependent satteningplastiaty, Int. J. Num. Meth. Engng, 39, 3731-3755 (1996).

[16]ZBIB, H. M.; AIfANTIS, C. E. On the localization and postocalization behavior of pastic deformation I. On the initiation of shear bands, Res. Mechanica, 23, 261-277 (1988).

[17]COMI, C.; BERTHAUd, Y .; BILLARD ON, R. On localization in ductilebrittlemateials under compressiveloadings, Eur. J. Mech. A/ Solids, 14(1), 19-43 (1995).

[18]BALBO, A. R. Contribuição à formlação matemática de moddos constitutivos para materiais com dano contínua Tese de D outorado, Escola de Engenharia de São Carlos, USP, (1998).

[19]Comi, C.; MaIer, G.; Perego, U. Generalized vaniable finite dement modding and extremum therems in stepvise hodonomic dastoplastiaty with intemal variables Comp. Meth. in Appl. Mech. and Eng., 96, 213-237 (1992).

[20]NADAI, A. Plastiaty, McG raw-Hill, New York (1931).

[21]ASARO, R.J. Mateial modding and failure modes in mtal plastiaty Mechanics of Materials, 4, 343-373 (1985).

[22] Perzyna, M.K.D .; Perzyna, P. Adiabatic shear band localization in dasticplastic singeorystalsInt. J. Solids Structures, v.30, n.1, 61-89 (1993).

[23] Olmstead, W.E.; Nemat,-NASSER, S.; NI, L. Shear bands as surfaces of descontinuity, J. Mech.. Phys. Sol., v.42, n.4, 697-709 (1994).

[24]Cedolin, L.; Dei Poli, S.; IoRI, I. tensile bahavior of conote J. Eng. Mech., ASCE, v.113, n.3, 431-449 (1987).

[25]Peerlings, R.H.J.; De Borst, R.; Brekelmans, W.A.M.; De VReE, J.H.P. Computational modelling of gradient-enhanced damage for fracture and fatigue problems. In Computational Plastiaty: Fundamentals and applications, D.R.J.O wen, E.Onate, E.Hinton (eds.), Complas IV, Pineridge-Press, Barcellona, 1, 975-986 (1995).

[26] de Borst, R.; Heeres, O.M.; Benallal, A. A gradient enhanced damage model: theory and computation. In: Computational Plastiaty: Fundamentals and applications, D.R.J.O wen, E.Onate, E.Hinton (eds.), Complas IV, PineridgePress, Barcellona, 1, 975-986 (1995). 
[27] Comi, C. A gadient damage mode for dynamic localization problens Rend. Sc. Istituto Lombardo, to appear (1997).

[28]Co MI, C.; D RIEMEIER, L. On gadient regulanization of damage modds, Third Euromech Solid Mechanics Conference, Estocolmo, Suécia (1997).

[29] DE BoRST, R.; MÜHLHAUS, B. Continuum models for discontinuous media, In: Fracture Processes in Concte, Rokk and Ceramics van Mier, J.G .M.; Rots, J.G.; Bakker, A. (eds.), 601-618 (1991).

[30] Steinmann, P.; Stein, E. Finite element localization analysis of micropolar strength degrading materials. In: Computer moddling of conctestructures, Mang, $\mathrm{H}$.; Bicanic, N.; de Borst, R. (eds.), 435-444 (1994).

[31]IORDACHE, M.-M.; Willam, K. Localization properties of 'non-symmetric' Cosserat formulations. In: Computational mechanics new trends and applications, Idelsohn, S.; O nate, E., D vorkin, E. (eds.), CIMNE, Barcelona (1998).

[32] O LIVER, J. A consistent dharadteistic length for smeared cadkingmodds Int. J. Num. Meth. Eng., v.28 (1989).

[33] De Borst, R.; SluYs, L. J.; MÜhlhaus, H.-B.; PAMIN, J. Fundamental issues in finitedements amalyses of localization of defomationEng. Comp., v.10, 99-121, (1993).

[34] MÜHLHAUS, B.; VARD O ULAK IS, I. Thethidkness of shear bands in granular mateials, Géotechnique, 37, 271-283 (1987).

[35] BAZANT, Z.P.; Why continuum damage is nonlocal: micromehanics arguments J. Eng. Mech., v.117, n.5, ASCE (1991).

[36]BAZANT, Z.P.; CEDOLIN, L. Continuum Stability of structures Elastic, Indastic, Fracture, andDamageTheries Oxford University Press (1991).

[37]de Borst, R.; Feenstra, P. H.; PAMIN, J.; SluYs, L. J. Some coment issues in computational methanics of conrese structures Proc. EURO-C 1994 Int. Conf. Computer Modelling of Concrete Structures, H. Mang et al. (eds), Pineridge Press, Swansea, 283-302 (1994).

[38]Comi, C.; Perego, U. On visco-damage models for concrete at high strain rates. In: Computational Plastiaty: Fundamentals and applications, D.R.J.O wen, E.O nate, E.Hinton (eds.), Complas V, CIMNE, Barcellona, 2, 1551-1555 (1997).

[39]Dubé, J.-F. ; Pijaudier-Cabot, G.; La Borderie, C. Rate dependent damage model for concrete- Wave propagation and localization. In Computer moddling of conctestructures Mang, H.; Bicanic, N.; de Borst, R. (eds.), 313-322 (1994).

[40]D ubÉ, J.-F.; Pijaudier-CABOT, G.; LA Borderie, C.; G LYNN, J. Ratedqpendent damagemodd for conctein dynamics ASCE J. Eng. Mech., 122, 939-947 (1994).

[41] NEED LEMAN, A. Material ratedapendenceand mesh sensitivity on localization prodems Comp. Meth. Appl.. Mech. Eng.,67,69-86 (1988).

[42] Clough, R. W. Dynamics of structures, McG raw-Hill, Inc. (1975)

[43]Whitham, G . B. Liner andnanliner waves John Wiley \& Sons, Inc. (1974). 
[44]BLAND, D. R. Wavetheary and applications Clarendon Press, Oxford -England, (1988).

[45]BAZANT, Z.P.; BELYTSCHKO, T. Wave propagation in a strain-sottening bar. exact solution J. Eng. Mech., v. 111, 3, 381-389 (1985).

[46]ACHENBACH, J. D. Wavepropagation in dasticsdids Elsevier Science Publishers, Amsterdam - NL, $17^{\mathrm{a}}$ edição (1993).

[47] GrafF, K. F. Wavemtionin dasticsdids Oxford University Press (1975).

[48]DE BORST, R.; MÜHLHAUS, B. Gradient-dependent plastidity: formilation and algoithmicaspets, Int. J. Num. Meth. Eng.,v.35, 521-539 (1992).

[49]BENALLAL, A.; TVERGAARD, V.; Nonlocal continum effets onbifurcationin theplane strain tension-compression test, J. Mech. Phys. Solids, 43, 741-770 (1995).

[50]COMI, C.; D RIEMEIER L. Localizzazionein mateiali descritti da un modalo a danno con gradientedd secondb equarto ordine atas do XI Convegno Italiano di Meccanica Computazionale, Trento, Itália, 75-78 (1998).

[51]Comi, C.; Perego, U. Fommlazione di dementi finiti dastoplastid in variabili genealizzate, AIMETA, Sesto Convegno italiano di meccanica computazionale (1991).

[52] CO RRADI, L. A displacement formulation for the finite dement dasticplastic prodem, Meccanica, 18, 77-91 (1983).

[53] CO RRADI, L. On compatible finitedement modds for dasticplasticanalyses Meccanica, September, 133-150 (1978).

[54] Co MI, C.; PEREG O, U. A unified approach for variationally consistent finite dements in dastoplasticity, Comp. Meth. in Appl. Mech. And Engng., 121, 323-344 (1995).

[55]ZIENKIEWICZ O.C.; TAYLOR R.L. Thefinitedement method, 4th edition, v. 1, Mc Graw-Hill, London (1991).

[56]ZIENKIEWICZ O.C.; TAYLOR R.L. Thefinitedement mathod, 4th edition, v. 2, Mc Graw-Hill, London (1991).

[57] ST O LARSKI, H.; BELYTSCHKO, T. Limitation principles for mixed finitedements based on the Hu-Washizu variational formulation Comp. Meth. In Appl. Mech. And Eng., 60, 195-216 (1987).

[58] MANG ASARIAN, O .L. Sdution of symmenic linear complementanity prddens by iterative mthoods J. Opt. Theor. Appl., 22, 465 (1977).

[59]PANG , J.S. On the convergene of a basiciterativem thod for theimpliat complementanity problem J. of O pt. Theory and Appl., v.37, n.2, June, 149-162 (1982).

[60]NOOR, A.M.; ZARAE, S. An iterative scheme for complementarity problems Eng. Anal.,v.3, n.4, 221-224 (1986).

[61]D IRKSE, S. P.; FERRIS, M. C. ThePath sover. a non-mondonestabilization schemefor mixed complementanity prdbens, Optimization Methods \& Software, 5, 123-156 (1995).

[62]Prager, W. The general theery of limit design Proceedings of the 8th Internat. Conf. on Appl. Mech., Instanbul, 2, 65-72 (1952). 
[63] MAZARS, J. Application de la méanique de l'endommagenent au compotement non linégire \& àla nupture du bóton de structure, Tese de D outorado, Paris 6, França (1984).

[64] MeYeR, R.; Ahrens, H. ; DUdDECK, H. Mateial modd for conmetein cadked and unaradked states J. of Engng. Mech., ASCE, v.120, n.9, September, 1877-1895 (1994).

\subsection{Bibliografia complementar em ordem alfabética}

[1] Aifantis, E.C. Thephysics of plasticdefomation Int. J. Plasticity, 3, 211-247 (1987).

[2] BEDFO RD, A.; D RUMHELLER, D. S. Introduction to dastic wave propagation John Wiley \& Sons, England, (1994).

[3] CARRERA, E. A study on arclenghtypemthools and their qperation failures illustrated by a simplemodd, Comp. \& Str., v.50, n.2, 217-229 (1994).

[4] CHEN, W.F.; ZHANG, H. Structural plasticity. Theery, proddems, and CAE sotware, Spring-Verlag New Y ork Inc., 1991.

[5] CO MI, C.; CO RIG LIANO , A. On uniqueness of the dynamic finitestep prodlemin gradent dependent softeningplastiaty, Int. J. Solids Struct., 33 (26), 3881-3902 (1996).

[6] Comi, C.; MAIER, G.; Pereg O, U. Moddli per dementi finiti eproprieàestremali de problem "donomo nd passo" in dastgdasticiàa vanabili inteme, ATTi, X Congresso Nazionale dell'Associazione Italiana di Meccanica Teorica ed Applicata, (1990).

[7] CRISFIELD, M. A. New solution for linear and non-linerr finite dement analysis The mathematics of finite elements and applications V, J. R. Whiteman (ed), Academic Press, London, 49-81 (1985).

[8] D ESOYER, T.; CORMERY, F. On uniqueness and localization in dasticdamagematerials, Int. J. Solids Structures, 31(5), 733-744 (1994).

[9] D RIE MEIER, L. Considkraçás sobrea fadiga emmtais eo compatamento do concto sob soliataçãoádica, Dissertação de Mestrado, EESC - USP, São Carlos (1995).

[10] O liver, J.; CERVERA, M.; MANZO LI, O. On theuse of $\mathrm{J}_{2}$ plastiaty modds for the smulation of $2 \mathrm{D}$ strong discontinuities in solids Computational plasticity Fundamentals and Applications, D.R.J. O wen, E. Oñate e H. Hinton (eds.), part1, p. 38-55, CIMNE, Barcelona, Espanha (1997).

[11] PeerlingS, R.H.J. Gradient damage for quasi-brittle mateials Dissertação de Mestrado, Delft - Holanda (1994).

[12] ProenÇA, S.P.B.; Alvares, M.S. Abait numerical aspets on the use of a continum damage modd to concte, Anais do XV CILAMCE - Congresso ibero latino americano sobre métodos computacionais para engenharia, v.2, 13291338 (1994).

[13] NAdAI, A. PlastiatyMcG raw-Hill, New Y ork (1931). 
[14] RALPH, D. Strateges for traaing Gldbel convergene of damped Nentan's mothod for nonsmoth equations, via path search, Mathematics of Operations Research, 19, 352389 (1994).

[15] RAMM, E. Strategies for traaing thenonlinear responsenear limit paints, Europe - US Workshop Kuhr G ermany, 63-89 (1980).

[16] Rizzi, E.; MaieR, G.; Willam, K. On failure indicatars in miltidissipative mateials Int. J. Solids Structures, Special issue in memory of J. Simo, to appear (1996).

[17] Ro BINSO N, S. M. Nomal maps indured by linerr transfomations Mathematics of Operations Research, 17, 691-714 (1992).

[18] Simo, J.C. Strain softening and dissipation: a unification of approaches. In: Cradking and damage strain localization and size effet, Mazars, J.; Bazant, Z.P. (eds), Elsevier, 440-461 (1988).

[19] SLYUS, L.J., DE BORST, R. Dispersive propeties of gadient-dapendent and rate dependent media, Mechanics of Materials, 18, 131-149 (1994).

[20] ZBIB, H. M.; AIFANTIS, C. E. On the localization and postocalization behavior of plasticdefomation II. On theedution and thidkness of shear band, Res. Mechanica, 23, 279-292 (1988b).

[21] ZBIB, H. M.; AifanTIS, C. E. On the localization and postocalization behavior of plastic deformation III. On the structure and vedoity of the Pottein - LeChatdier bands, Res. Mechanica, 23, 293-305 (1988c). 


\section{Discussão sobre problema bem posicionado}

Introdução

$\mathrm{Na}$ formulação diferencial de um problema, estabelecemos o equilíbrio e as relações constitutivas de elementos diferenciais em termos das variáveis de estado. Isto leva a um sistema de equações diferenciais onde é possível que todas as condições de compatibilidade, contorno e valor inicial estejam contidas nessas equações.

O problema resulta bem posicionado se:

$\checkmark$ existe ao menos uma solução linearmente independente

$\checkmark$ esta solução é única

$\checkmark$ a solução depende continuamente dos dados

Se uma das condições é violada, o problema resulta mal posicionado. Em uma análise matemática do bom posicionamento, deve-se classificar as equações diferenciais governantes relacionadas ao problema físico que estas representam.

Classificação das equações diferenciais a derivadas parciais de segunda ordem

Considera-se equações diferenciais parciais de segunda ordem no domínio $(\mathrm{x}, \mathrm{y})$, de modo que: 
$A(x, y) \frac{\partial^{2} u}{\partial x^{2}}+2 B(x, y) \frac{\partial^{2} u}{\partial x \partial y}+C(x, y) \frac{\partial^{2} u}{\partial^{2} y}=\phi\left(x, y, u, \frac{\partial u}{\partial x}, \frac{\partial u}{\partial y}\right)$

onde ué a variável de estado desconhecida.

D ependendo dos coeficientes em A.1 a equação diferencial é elíptica, parabólica ou hiperbólica,

$\mathrm{B}^{2}-\mathrm{AC} \begin{cases}<0 & \text { díptica } \\ =0 & \text { parabóica } \\ >0 & \text { hiperbólica }\end{cases}$

As diferenças entre as três categorias de expressões são aparentes quando relacionadas com os problemas físicos que elas governam. Na forma mais simples os três tipos de equações podem ser identificadas com equação de Laplace, equação de condução de calor e ๕quaçãodeonda, respectivamente.

Em problemas elípticos, os valores das variáveis de estado desconhecidas (ou suas derivadas) são dadas no contorno. Estes problemas são, por esta razão, chamados de problemas de valor de contorno. Assim, a solução em um ponto qualquer interior depende dos dados de cada ponto do contorno. Equações diferenciais elípticas governam a resposta estática do sistema.

Em contraste com a equação elíptica, as equações parabólicas e hiperbólicas incluem tempo como uma variável independente e, portanto, definem problemas de propagação. Estes problemas são também chamados de problemas de valor inicial porque a solução depende das condições iniciais.

Modelos de dano com gradientes de segunda e quarta ordem

Para o caso do modelo com gradientes de ordens superiores tem-se um sistema de equações diferenciais nas variáveis plásticas e velocidade. Para verificação do bom posicionamento sugere-se Benallal $\&$ Tvergard ${ }^{49}$ no caso de consideração somente de gradiente de segunda ordem. 
Para o modelo de plasticidade apresentado neste trabalho, que envolve termos com gradientes até quarta ordem, o sistema de equações diferenciais, correspondente ao caso estático, é formado por:

$\mathrm{f}=\dot{\sigma}-\mathrm{h}^{\dot{ }}+\mathrm{G}_{1} \nabla^{2} \dot{\lambda}-\mathrm{C}_{2} \nabla^{4} \dot{\lambda}=0$

$\operatorname{div}(\dot{\sigma})=\operatorname{div}(E \nabla \dot{u}-E \dot{\lambda} n)=0$

Considera-se as partes principais do sistema das equações A.2 e A.3 (Benallal \& Tvergard ${ }^{49}$ ), respectivamente:

$-\mathrm{C}_{2} \nabla^{4} \lambda^{\cdot}=0$

$\operatorname{div}(\operatorname{E} \nabla \dot{\mathrm{u}})=0$

A matriz característica do sistema é dada por:

$$
M=\left[\begin{array}{cc}
E(N \otimes N) & 0 \\
0 & -\mathrm{C}_{2}(\mathrm{~N} \cdot \mathrm{N})
\end{array}\right]
$$

O problema em questão deixa de ser bem posicionado ou perde a elipticidade, quando 0 determinante da matriz se anula, para $\mathrm{N} \neq 0$ :

$\mathrm{C}_{2}(\mathrm{~N} \cdot \mathrm{N}) \operatorname{det}[\mathrm{E}(\mathrm{N} \otimes \mathrm{N})]=0$

Por outro lado, é possível analisar a estabilidade da solução localizada também pelo sinal do determinante da matriz característica. No caso, as soluções localizadas tem as formas:

$$
\dot{\mathrm{u}}=\dot{\mathrm{u}}_{0} \mathrm{e}^{\mathrm{ign} \cdot \mathrm{x}}, \quad \dot{\lambda}=\dot{\lambda}_{0} \mathrm{e}^{\mathrm{ign} \cdot \mathrm{x}}
$$

e a matriz característica resulta:

$$
\mathrm{M}=\left[\begin{array}{cc}
\mathrm{C}_{2} \mathrm{q}^{4} & 0 \\
0 & \mathrm{Eq}^{2}
\end{array}\right]
$$

Para a forma característica A.9, o determinante é sempre positivo, já que $\mathrm{c}_{2}>0 \mathrm{e}$ $\mathrm{E}>0$. Q uando o parâmetro $\mathrm{C}_{2}$ é nulo (somente gradiente segundo), $\mathrm{G}>0$ garante a elipticidade. Portanto, justificam-se os sinais contrários de G e $\mathrm{C}_{2}$. 


\section{Elementos finitos com variáveis generalizadas}

\section{Introdução}

Neste trabalho, emprega-se na discretização o Método dos Elementos Finitos na sua formulação em vanáveis generalizadas A noção de variáveis generalizadas foi primeiramente introduzida por Prager ${ }^{62}$. 0 assunto é discutido mais tarde por Corradi ${ }^{52,53}$, Comi \& al. ${ }^{19}$, Comi \& Peeeg ${ }^{15}$. Este Apêndice contém informações resumidas sobre a teoria desta discretização.

Ao Pditemicodi Milano onde foi estudada a parte numérica deste trabalho, o método está implementado no programa de Elementos Finitos de Zienkievicz \& Tayłar ${ }^{55,56}$.

Variáveis generalizadas

Variáveis generalizadas podem ser entendidas como valores representativos de deslocamentos, deformações, tensões, ou outras variáveis internas obtidos por integrais ponderadas no domínio do elemento. As funções de ponderação de cada variável podem, a princípio, ser quaisquer e independentes.

Introduz-se matrizes de interpolação $\mathrm{N}$ para 0 campo de deformações $\varepsilon$ e tensões $\sigma$,

$\varepsilon(\mathrm{x})=\mathrm{N}_{\varepsilon} \bar{\varepsilon}, \quad \sigma(\mathrm{x})=\mathrm{N}_{\sigma} \bar{\sigma}, \quad \mathrm{x} \in \Omega$

onde $\bar{\varepsilon}$ e $\bar{\sigma}$ representam os vetores de variáveis generalizadas para o elemento $\Omega$. 
Segundo Conad ${ }^{52}$, para que a solução do problema elástico resulte da minimização de um funcional com respeito aos campos de deslocamentos, tensões e deformações (cada um desses campos discretizado com funções de interpolação independentes), as vanávés genealizadas devem se conforme $\operatorname{Prager}^{62}$ na forma de pares conjugados.

Os pares conjugados são definidos pela equivalência com produto interno:

$\bar{\sigma} \cdot \bar{\varepsilon}=\int_{\Omega} \sigma \cdot \varepsilon d \Omega \quad \forall \bar{\sigma}, \bar{\varepsilon}$

Substituindo-se as equações (B.1) em (B.2), tem-se as seguintes relações:

$$
\begin{array}{ll}
\bar{\sigma} \cdot \bar{\varepsilon}=\bar{\sigma}^{\mathrm{T}} \int_{\Omega} \mathrm{N}_{\sigma}^{\mathrm{T}} \varepsilon \mathrm{d} \Omega=\bar{\varepsilon}^{\mathrm{T}} \int_{\Omega} \mathrm{N}_{\varepsilon}^{\mathrm{T}} \sigma \mathrm{d} \Omega=\bar{\sigma}^{\mathrm{T}} \int_{\Omega} \mathrm{N}_{\sigma}^{\mathrm{T}} \mathrm{N}_{\varepsilon} \mathrm{d} \Omega \bar{\varepsilon} & \forall \bar{\sigma}, \bar{\varepsilon} \\
\bar{\varepsilon}=\int_{\Omega} \mathrm{N}_{\sigma}^{\mathrm{T}} \varepsilon \mathrm{d} \Omega, \quad \bar{\sigma}=\int_{\Omega} \mathrm{N}_{\varepsilon}^{\mathrm{T}} \sigma \mathrm{d} \Omega, \quad \int_{\Omega} \mathrm{N}_{\sigma}^{\mathrm{T}} \mathrm{N}_{\varepsilon} \mathrm{d} \Omega=1
\end{array}
$$

As equações B.4a,b expressam as variáveis generalizadas como integrais ponderadas dos campos mencionados anteriormente, usando-se como pesos as interpolações do campo conjugado.

A equação B.4c apresenta uma condição de interdependência entre as duas interpolações. Esta condição é satisfeita se, por exemplo, depois de haver arbitrariamente escolhido o modelo de deformação $\mathrm{N}_{\varepsilon}$, 0 campo de tensões é modelado pela matriz de interpolação:

$$
\mathbf{N}_{\sigma}=\mathbf{N}_{\varepsilon}\left[\int_{\Omega} \mathbf{N}_{\varepsilon}^{\mathrm{T}} \mathbf{N}_{\varepsilon}\right]^{-1}
$$

De B.4c e B.5, segue que:

$$
\int_{\Omega} \mathrm{N}_{\varepsilon}^{\mathrm{T}} \mathrm{N}_{\varepsilon} \mathrm{d} \Omega=\left(\int_{\Omega} \mathrm{N}_{\sigma}^{\mathrm{T}} \mathrm{N}_{\sigma} \mathrm{d} \Omega\right)^{-1}, \quad \mathrm{~N}_{\varepsilon}=\mathrm{N}_{\sigma}\left[\int_{\Omega} \mathrm{N}_{\sigma}^{\mathrm{T}} \mathrm{N}_{\sigma} \mathrm{d} \Omega\right]^{-1}
$$


O utra alternativa seria escolher interpolações de modo que:

$$
\begin{aligned}
& \int_{\Omega} \Omega^{-1} \mathrm{~N}_{\sigma}^{\mathrm{T}} \mathrm{N}_{\varepsilon} \mathrm{d} \Omega=\Omega^{-1} \mathrm{I} \\
& \therefore \mathrm{N}_{\sigma}^{\mathrm{T}} \mathrm{N}_{\varepsilon}=\Omega^{-1} \mathrm{I}
\end{aligned}
$$

Pode-se seguir o mesmo caminho para o par de variáveis incremento de dano $\dot{D}$ e força termodinâmica associada ao dano $\mathrm{Y}$. Adaptando-se sobre 0 elemento $\Omega$ as interpolações:

$$
\dot{\mathrm{D}}(\mathrm{x})=\mathrm{N}_{\mathrm{D}}(\mathrm{x}) \dot{\mathrm{D}}, \quad \mathrm{Y}(\mathrm{x})=\mathrm{N}_{\mathrm{Y}}(\mathrm{x}) \overline{\mathrm{Y}}
$$

A equivalência de produto interno resulta:

$$
\dot{\mathrm{D}} \overline{\mathrm{Y}}=\int_{\Omega} \dot{\mathrm{D}}(\mathrm{x}) \mathrm{Y}(\mathrm{x}) \mathrm{d} \Omega \quad \forall \dot{\mathrm{D}}, \overline{\mathrm{Y}}
$$

As seguintes equações são equivalentes a B.4a,b e B.5:

$$
\dot{\overline{\mathrm{D}}}=\int_{\Omega} \mathrm{N}_{\mathrm{Y}}^{\mathrm{T}} \dot{\mathrm{D}} \mathrm{d} \Omega \quad \overline{\mathrm{Y}}=\int_{\Omega} \mathrm{N}_{\mathrm{Y}}^{\mathrm{T}} \mathrm{Y} \mathrm{d} \Omega \quad \mathrm{N}_{\mathrm{Y}}=\mathrm{N}_{\mathrm{D}}\left[\int_{\Omega} \mathrm{N}_{\mathrm{D}}^{\mathrm{T}} \mathrm{N}_{\mathrm{D}} \mathrm{d} \Omega\right]^{-1}
$$

